\title{
Preoperative optimization of diagnostic work-up and physical fitness predicts and improves outcome of patients with colorectal cancer
}

Citation for published version (APA):

Berkel, A. E. M. (2022). Preoperative optimization of diagnostic work-up and physical fitness predicts and improves outcome of patients with colorectal cancer. [Doctoral Thesis, Maastricht University]. ProefschriftMaken. https://doi.org/10.26481/dis.20220120ab

Document status and date:

Published: 01/01/2022

DOI:

10.26481/dis.20220120ab

Document Version:

Publisher's PDF, also known as Version of record

Please check the document version of this publication:

- A submitted manuscript is the version of the article upon submission and before peer-review. There can be important differences between the submitted version and the official published version of record.

People interested in the research are advised to contact the author for the final version of the publication, or visit the DOI to the publisher's website.

- The final author version and the galley proof are versions of the publication after peer review.

- The final published version features the final layout of the paper including the volume, issue and page numbers.

Link to publication

\footnotetext{
General rights rights.

- You may freely distribute the URL identifying the publication in the public portal. please follow below link for the End User Agreement:

www.umlib.nl/taverne-license

Take down policy

If you believe that this document breaches copyright please contact us at:

repository@maastrichtuniversity.nl

providing details and we will investigate your claim.
}

Copyright and moral rights for the publications made accessible in the public portal are retained by the authors and/or other copyright owners and it is a condition of accessing publications that users recognise and abide by the legal requirements associated with these

- Users may download and print one copy of any publication from the public portal for the purpose of private study or research.

- You may not further distribute the material or use it for any profit-making activity or commercial gain

If the publication is distributed under the terms of Article 25fa of the Dutch Copyright Act, indicated by the "Taverne" license above, 


\section{Preoperative optimization of diagnostic work-up and physical fitness predicts and improves outcome of patients with colorectal cancer}


Cover design: Stefanie van den Herik | HerikMedia | www.herikmedia.nl Lay-out: Stefanie van den Herik | HerikMedia | www.herikmedia.nl Printed by: ProefschriftMaken | www.proefschriftmaken.nl

ISBN: 978-94-6423-578-4

C) 2021 Annefleur Elisabeth Maria Berkel, The Netherlands. All rights reserved. No parts of this thesis may be reproduced, stored in a retrieval system or transmitted in any form or by any means without permission of the author. Alle rechten voorbehouden. Niets uit deze uitgave mag worden vermenigvuldigd, in enige vorm of op enige wijze, zonder voorafgaande schriftelijke toestemming van de auteur. 


\section{Preoperative optimization of diagnostic work-up and physical fitness predicts and improves outcome of patients with colorectal cancer}

\section{Proefschrift}

ter verkrijging van de graad van doctor aan de Universiteit Maastricht, op gezag van de Rector Magnificus, Prof.dr. Rianne M. Letschert volgens het besluit van het College van Decanen, in het openbaar te verdedigen op donderdag 20 januari 2022 om 13.00 uur

door 


\section{Promotores}

Prof.dr. N.L.U. van Meeteren

Prof.dr. J.M. Klaase (Universitair Medisch Centrum Groningen)

\section{Copromotor}

Dr. B.C. Bongers

\section{Beoordelingscommissie}

Prof.dr. W.F.F.A. Buhre (voorzitter)

Prof.dr. C.H.C Dejong

Prof.dr. L.P.S. Stassen

Prof.dr. C. Veenhof (Universitair Medisch Centrum Utrecht)

Prof.dr. C. Verhoef (Erasmus Medisch Centrum) 


\section{Contents}

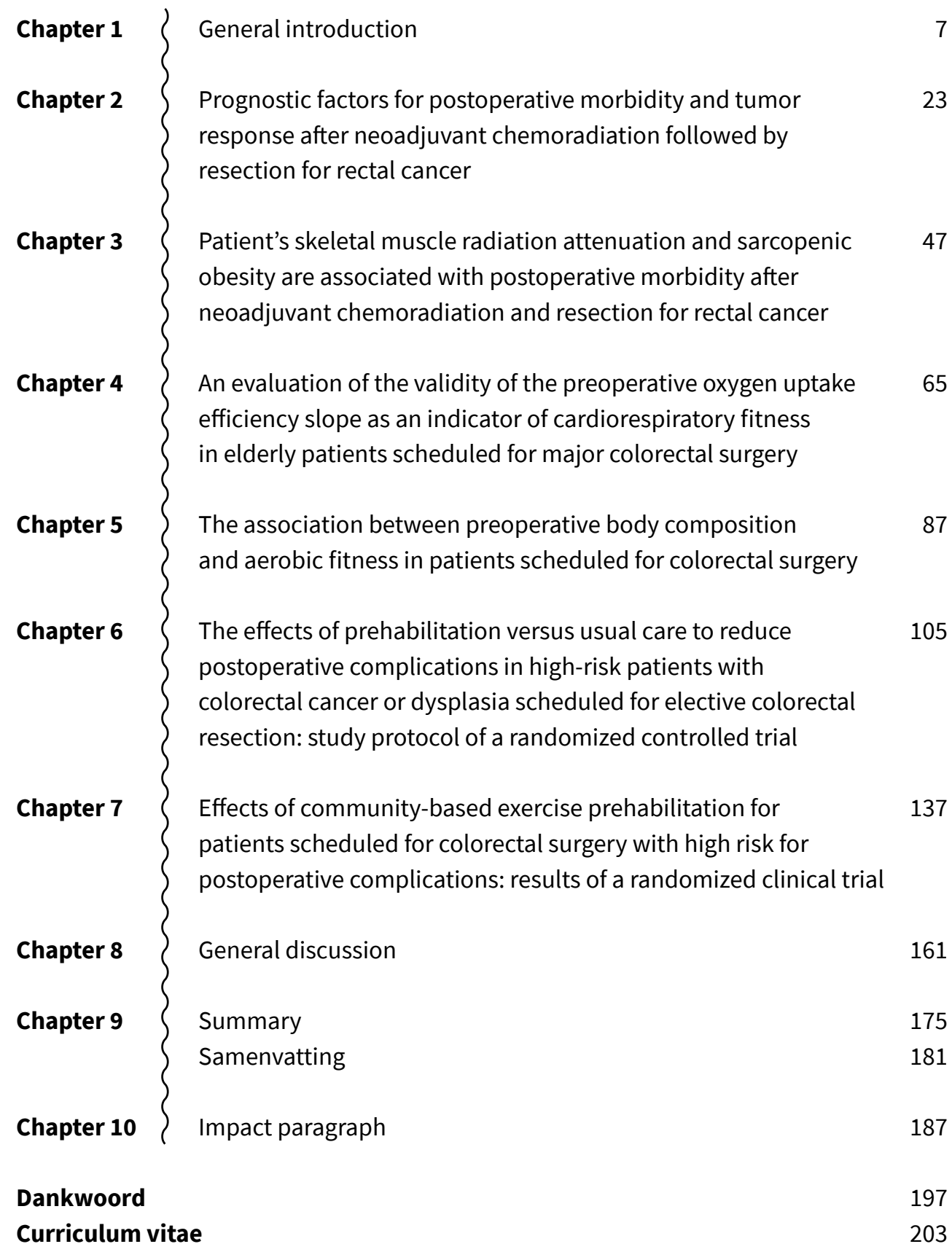





\section{Introduction}

\section{General background}

The ambition of the Dutch Ministry of Health, Welfare and Sport is to keep everyone healthy as long as possible and to restore the sick to health as quickly as possible. ${ }^{1}$ Overall life expectancy continues to increase, as, among a lot of other permissive socio-cultural developments, public health and medicine are improving. Thereby, the number of the elderly in the population will increase simultaneously. According to the World Health Organization, one in four of those living in Europe will be over 65 years of age by 2050, versus $13 \%$ in $1997 .^{2}$ The oldest-old population, people 80 years of age and older, forms a relatively small proportion of the current population, but is now relatively the fastest growing population segment. ${ }^{2}$

With old age come ailments, which makes prevention and treatment more challenging. In the elderly, values such as vital functioning in daily life, full participation in society, and wellbeing become increasingly important. ${ }^{3}$ In addition to prevention of disease, prevention of limitations in daily functioning and societal participation is necessary ('function-oriented prevention'). ${ }^{3}$ In this form of prevention, it is all about identifying determinants that can be influenced positively to prevent disability, independently of, or in addition to, a disease-oriented approach. ${ }^{3}$ This is in accordance with the ambition of the Dutch Ministry of Health, Welfare and Sport for 2024 to ensure that a larger proportion of elderly will feel themselves vital so that they can (according to ability) remain able to participate in society. ${ }^{4}$ This also fits with the new definition of health as the ability to adapt and self-manage, in the face of social, physical, and emotional challenges, ${ }^{5}$ and with the concept of 'positive health'. ${ }^{6}$

With the rise of the aging population, the incidence of cancer will also increase. In many cancer types, surgery is the cornerstone of treatment. As a result, the number of patients undergoing major surgery every year is likely to increase. Every person will undergo approximately six surgical procedures in their lifetime, and therefore, statistically, we are all preoperative. ${ }^{7}$ Psychophysiological function and reserves of multiple organ systems gradually decline with aging, which affects the resilience to surgery, thereby making especially elderly more vulnerable to both pre- and postoperative complications., Not only the focus of the health-system is moving towards prevention (primary, secondary, and tertiary prevention), as can be noticed from the recent instalment of national screening programs (e.g., for colorectal-, breast-, and cervical cancer); also in perioperative care, there is an increased focus on prevention (e.g., the prevention of (the impact of) postoperative complications, prevention of a delayed or even permanent loss of (physical) functioning). Methods to determine risk profiles in order to identify at-risk groups for clinical trials, and even personal risk-profiles of individual persons for regular care, are essential for personalized and successful prevention. 
In function-oriented prevention, prehabilitation fits well. Although prehabilitation was mentioned for the first time in a scientific publication in 1946 (which focused on the improvement of the physical fitness level of young recruits of the British Army), ${ }^{10}$ prehabilitation gained rather unsuccessful interest in the 80-90's of the former century and is again and more focused gaining great interest in perioperative care since the beginning of this century. Prehabilitation may be defined as a process of stimulation of resilience in the continuum of health along the patient journey that occurs between the time of disease diagnosis and the beginning of curative treatment.9,11 Prehabilitation includes physical and psychosocial screening and assessments that establish a person's baseline functional level as soon as possible after diagnosis, identifies his or her talents, capacities, socio-cultural context, and, on top of these, impairments., ${ }^{9,11,12}$ Moreover, prehabilitation provides targeted preventive interventions that may assist to improve a patient's health and daily functioning preoperatively, in order to try to reduce the incidence and the severity of current and future - also postoperative - impairments. ${ }^{9,11}$ As such, prehabilitation may also provide an opportunity to assist patients to get as quickly as possible to their best personal level of functioning pre- and especially post-surgery, thereby reducing the risks of surgery-related morbidity and/or mortality, decreasing the length of stay in the hospital and the episode of rehabilitation, and even reducing readmissions. ${ }^{9}$ In short, prehabilitation aims to optimize modifiable risk factors before commencement of treatment in order to prevent or reduce the severity of anticipated treatment-related impairments that may cause significant disability, (co-) morbidities, or even mortality.

\section{Colorectal cancer}

Colorectal cancer is the third most common cancer worldwide. An estimated 1.8 million new cases diagnosed and 881,000 deaths are associated with the disease in $2018 .{ }^{13}$ In the Netherlands, the incidence of colorectal cancer more than doubled in the recent years, with an incidence of over 14,000 in $2018 .{ }^{14}$ In the Netherlands, more than $65 \%$ of the patients with colorectal cancer are older than 65 years. ${ }^{15}$

Early diagnosis and prognosis, followed by surgical resection of the colorectal tumor, remains the foundation of curative treatment. ${ }^{16}$ In patients with locally advanced rectal cancer, neoadjuvant chemoradiotherapy followed by surgery has become the standard of care. ${ }^{17,18}$ In patients with rectal cancer who achieve a complete clinical response after neoadjuvant therapy, the strategy of "watch and wait" is a new strategy that offers an opportunity for patients to avoid major resection surgery..$^{18}$ Twenty-five percent of the patients with a complete clinical response after neoadjuvant therapy and treated with a "watch and wait" approach developed a local regrowth in the first two years of follow-up, emphasizing the importance of endoscopic surveillance to ensure the option of deferred curative surgery. ${ }^{18}$ 
Over the years, laparoscopic surgery is increasingly used as standard of care for colorectal cancer resection and is nowadays considered as the approach of choice for primary colorectal resection. ${ }^{19}$ Between 2011 and 2015, the use of laparoscopy increased from 55 to $84 \%$ in colon cancer, and from 49 to $89 \%$ in rectal cancer. Conversion rates decreased from 11.8 to $8.6 \%$ and from 13.0 to $8.0 \%$, respectively. ${ }^{20}$ After resection for colon or rectal carcinoma, respectively $15 \%$ and $20 \%$ of the patients have a complicated course (i.e. a prolonged hospital stay of $>14$ days, reintervention, or mortality) within 30 days after surgery..$^{16}$ This complication rate did not obviously reduce over the last years. ${ }^{16,21}$ However, surgical complications seemed to become less severe in both colon and rectal cancer surgery and probably better managed since reintervention rates, length of hospital stay, and mortality rates decreased. ${ }^{21}$ Studies showed that laparoscopic resection reduced the risk of postoperative mortality compared with open resection ${ }^{22}$ and that increased surgical specialization contributes to the improvement of long term survival following colorectal cancer surgery. ${ }^{23}$ Moreover, the implementation of "enhanced recovery after surgery" (ERAS) programs resulted in major improvements in clinical outcomes and cost. ${ }^{24}$ Clinical auditing might also contribute to improvements in outcomes. ${ }^{21}$ In 2014 , a national colorectal screening program was introduced in the Netherlands, and it was shown that with help of this "early warning system" postoperative outcomes are better for people diagnosed with colon cancer through the screening program compared with nonscreen-detected patients. ${ }^{25,26}$ Despite these improvements, the overall complication rate (with percentages up to $30 \%)^{16,27,28}$ in colorectal surgery remains significant, especially in the fraction of patients that are diagnosed outside the screening program. A complicated postoperative course may delay recovery, prolongs hospitalization, causes unplanned hospital readmission and chronic illness, severely impairs short- and long-term physical functioning and health-related quality of life, ${ }^{29}$ and diminishes overall ${ }^{30}$ and cancerspecific survival. ${ }^{30,31}$

\section{Risk assessment}

Improving daily functioning while reducing the number and impact of complications would considerably reduce patient burden and societal costs. ${ }^{27}$ Preoperative screening and assessment for risk factors might assist to timely identify patients at high and low risk of postoperative complications, and - mostly coincided - functional decline. ${ }^{9}$ Preoperative risk assessment is a prerequisite for clinical reasoning and shared decisionmaking for the choice and use of prophylactic, surgical, and anesthetic interventions. By doing so, treatment can potentially effectively be focused on and tailored with highrisk patients, with attention on (partially) modifiable risk factors, in order to decrease their risk for adverse short- and long-term postoperative outcomes. In accordance to this, Bates and colleagues ${ }^{32}$ proposed a stepwise framework for re-engineering local perioperative services, aimed at transitioning between screening (by using validated self-reported questionnaires and/or practical functional performance tests), assessment 
(e.g. by using a sophisticated cardiopulmonary exercise test), and preventive intervention (prehabilitation), enabling multilevel benefits of tailored risk assessment and personalized interventions.

The nationwide Dutch ColoRectal Audit (DCRA), in which data on various patient, tumor, treatment, and short-term (30-day) outcome characteristics are collected of patients undergoing a resection for primary colorectal cancer in the Netherlands, enables hospitals to compare their quality of colorectal outcome and thereupon infer options to improve the quality of care, after correction for case-mix factors. De Neree tot Babberich and colleagues ${ }^{21}$ evaluated the achievements made in colorectal cancer surgery since the start of the DCRA, and showed an overall improvement of outcomes, without signs of risk averse behavior (e.g., patient selection). The case-mix factors used, are sex, body mass index (BMI), age, Charlson comorbidity score, American Society of Anesthesiologists (ASA) classification, preoperative tumor complications, urgency of the resection, tumor location, extensive resection due to locally advanced tumors or metastases, pathologic tumor (pT) stage, and pathologic metastasis (pM) stage..$^{33}$ However, there might be more powerful pre- and postoperative risk factors that could identify high-risk patients, and thereupon enabling patients to make better informed decisions before and after surgery and personalized interventions. Moreover, these more powerful perioperative risk factors, such as habitual level of physical activity, muscle mass, muscle density (also known as skeletal muscle radiation attenuation or myosteatosis), and aerobic fitness could potentially be used as case-mix correction factors.

In recent years, a patient's body composition, i.e. the relative proportions of subcutaneous adipose tissue, visceral adipose tissue, skeletal muscle quantity and density, has extensively been studied as a predictor for outcome following oncological treatment. ${ }^{34}$ Sarcopenia, indicated by the involuntary, age-dependent, depletion of skeletal muscle mass and reduction of skeletal muscle function, ${ }^{35}$ is associated with more infectious complications, ${ }^{36}$ higher mortality, ${ }^{37}$ and decreased surviva ${ }^{38}$ after colorectal cancer resection. Sarcopenia can reliably and validly amongst others be assessed by measurement of muscle crosssectional area most often determined at the level of the third lumbar vertebra (L3) by using the computed tomography (CT) scan. ${ }^{39-42}$ Sarcopenic obesity is found to be associated with severe postoperative complications following open colon cancer surgery, ${ }^{43}$ and an independent predictor of survival in patients with solid tumors in the respiratory or gastrointestinal tract. ${ }^{34}$ Low skeletal muscle radiation attenuation represents a high amount of intramuscular adipose tissue (myosteatosis) and is independently prognostic of survival of patients with cancer. ${ }^{42} \mathrm{~A}$ lower radiation attenuation was also found to be the best predictor of any complication after colectomy for colon cancer, ${ }^{44}$ and was associated with overall complications after open colon resection for cancer. ${ }^{43}$ Since a CT scan is part of usual care guidelines/protocol and consequently routinely performed as an integrated 
part in the work-up of all patients with colorectal cancer, no additional test is needed to obtain and evaluate body composition measurements.

Moreover, preoperative aerobic fitness, assessed by cardiopulmonary exercise testing (CPET), is a powerful preoperative risk assessment tool that has consistently been found to be able to independently predict postoperative outcome in patients undergoing intraabdominal surgery. ${ }^{45}$ Patients with a low preoperative aerobic fitness (oxygen uptake at the ventilatory anaerobic threshold (VAT) $<11 \mathrm{~mL} / \mathrm{kg} / \mathrm{min}$ ) are particularly at increased risk of postoperative complications. ${ }^{45,46}$ Peak oxygen uptake and oxygen uptake at the VAT are the most commonly used preoperative cardiopulmonary exercise test measures in studies recorded before major elective abdominal surgery. ${ }^{45}$ Since there are several clinical limitations when determining aerobic fitness using peak oxygen uptake and/or oxygen uptake at the VAT, and since assessing only these two measures leads to substantial loss of physiological data, alternative objective (sub)maximal variables of aerobic fitness (e.g., the oxygen uptake efficiency slope [OUES]) might be useful for preoperative risk assessment. The OUES has been reported to have prognostic value in predicting major cardiac events and mortality in patients with heart failure and coronary artery disease. ${ }^{47-50}$

Despite its usefulness in perioperative medicine, CPET is relatively expensive, timeconsuming, requires well-trained personnel for an adequate interpretation of its results, and is currently not used as standard risk assessment tool in daily practice. As muscle mass is related to aerobic fitness, ${ }^{51}$ the question remains whether body composition parameters on CT scan could help to diagnose patients with low preoperative fitness, to preselect for CPET.

\section{Prehabilitation}

High-risk, less physically fit patients might especially benefit from preoperative exercise training (prehabilitation) to optimize their muscular and aerobic fitness in order to facilitate a prompt recovery of physical functioning and in parallel reduce their risk of a staggered and even medically complicated postoperative course. ${ }^{52,53}$ However, to date, studies have provided convincing data and evidence that prehabilitation does not reduce postoperative complications in patients with a low risk of postoperative complications. Additional critics considering these studies are that they tended to be underpowered, heterogeneous, and possessed highly imperfect therapeutic approaches. ${ }^{29,53-56}$ Adequate risk assessment, to select patients who would benefit the most from prehabilitation, that are probably patients with high-risk profiles for postoperative complications, seems therefore a more logical next step in experiments. This step follows almost exactly what has been the route the former decades in thoracic, especially open heart, surgery. Up until 2000 here almost all trials demonstrated a negative cost-effectiveness from prehabilitation, 
whereas the first adequately powered trial focusing on high-risk coronary artery bypass graft (CABG) patients demonstrated the potential of this remedy. ${ }^{57}$

According to the modified conceptual model of Topp and colleagues (see Figure 1), ${ }^{58}$ a patient's functional capacity tends to decline postoperatively. Patients with a poor preoperative functional capacity may decline to the point where the individual is unable to maintain independent functioning after surgery (critical zone). These patients are less able to successfully cope with hospitalization and surgery and are at high risk of postoperative morbidity and even mortality. Figure 1 therefore shows a schematic conceptual hypothesis of the possible effects of prehabilitation on performance status in patients undergoing surgery, here typically colorectal surgery.

\section{Prehabilitation}

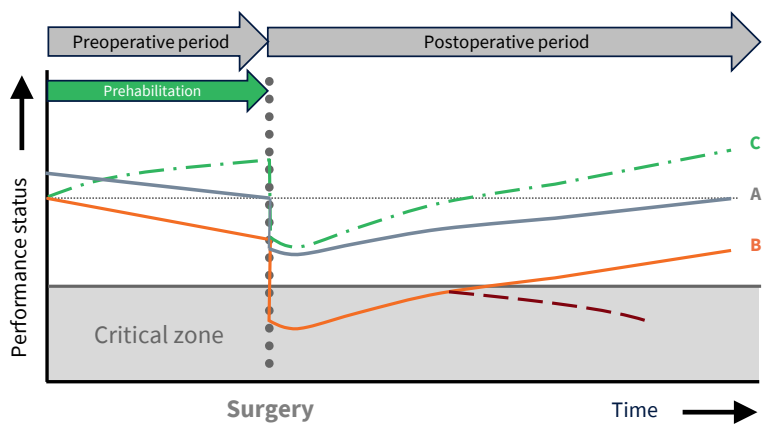

Figure 1. Conceptual hypothesis of the possible effects of prehabilitation on performance status and postoperative recovery in patients undergoing colorectal surgery. Adapted and modified from: Topp et al. $2002^{58}$ and Bongers et al. 2020. ${ }^{66}$

A: Patients with a good preoperative performance state (low-risk patients, grey line) are able to cope with the surgical stress response, and do not enter the critical zone.

B: Patients with a poorer preoperative performance status (high-risk patients, orange line) are not able to cope with the surgical stress response and do enter the critical zone putting them at high risk of postoperative morbidity. Some of these patients (dark red, dashed line) are not able to leave this critical zone and therefore might not survive.

C: Prehabilitation in high-risk patients (green, dashed line) aims to preoperatively increase a high-risk patient's physical performance status, thereby increasing adaptive capacity to cope with the severe diagnosis, eventually neoadjuvant treatment, hospitalization, the surgical stress response and reduce the risk of morbidity and mortality.

A prehabilitation program may include physical exercise training, nutritional support, and psychological support (uni- or multimodal program). Exercise prehabilitation must start as soon as possible in order to take advantage as much as possible of the normally asymptotic response of the cardiovascular and musculoskeletal system upon physical exercise training in the mostly relatively short interval between diagnosis and surgery. ${ }^{9}$ 
For an exercise program to be considered therapeutically valid, the use of the CONTENTscale is suggested. ${ }^{59}$ Barberan-Garcia and colleagues ${ }^{60}$ reported that prehabilitation prior to elective major abdominal surgery significantly improved preoperative aerobic fitness and significantly reduced the number of high-risk patients (based on age and ASA score) with postoperative complications, as compared to usual care. The recently published study of Carli et $\mathrm{al}^{56}$ demonstrated that prehabilitation in (pre)frail patients (based on the Fried frailty index) opting for resection of colorectal cancer did not reduce postoperative complications, a finding that is consistent with former findings for low-risk populations. ${ }^{61}$ Thomas and colleagues ${ }^{53}$ suggested that future prehabilitation research should focus more on the adequate and objective selection of high-risk surgical patients (e.g., by performing a CPET) and provide personalized, and probably multimodal, (partly) supervised prehabilitation at home or in a community-based setting with objective high frequency monitoring (named 'titration'62) of a patient's asymptotic progress of fitness. Ideally, a preoperative exercise program must be integrated in the perioperative trajectory and performed in the patient's pre-existent living context (home-based or communitybased prehabilitation), to maximize participation rate, adherence, and effectiveness. Moreover, they concluded that measuring not only the incidence but also the impact of postoperative complications may contribute to a more nuanced demonstration of the clinical and economic value of prehabilitation..$^{53}$

The timeframe between the decision for colorectal surgery and the actual surgery is limited. However, based on the aforementioned developments in high-risk CABG patients who demonstrate risk reduction after at least 14 days of preoperative intensive inspiratory muscle training, ${ }^{57}$ this timeframe still seems to be adequate if the continuum of health and care are logistically and time wise optimally organized. The largest improvements in fitness occur in the first weeks of physical exercise training, where after further improvements gradually level off over time. ${ }^{63}$ Dutch guidelines describe that treatment of patients with a colorectal tumor will ideally start within six weeks after their first visit at the outpatient clinic. ${ }^{64}$ Therefore, the valuable available time for prehabilitation seems quite adequate, but still somewhat short. Given the need for prehabilitation interventions to be both effective and time-efficient, Weston et al. ${ }^{65}$ indicated that carefully designed and supervised high-intensity interval training programs might be a promising perioperative strategy for enhancing aerobic fitness within a short time period. Besides, logistics should be optimized to ensure prompt screening and assessment for modifiable patient-related risk factors. Not only physical fitness level, but also nutritional status, iron deficiency anaemia, psychological resilience, and intoxications (alcohol and smoking behavior) on the one hand. ${ }^{66}$ On the other hand, hospital planning and logistics should be re-evaluated, thereby taking the medical urgency to operate into account, in order to plan the neoadjuvant and surgical procedures keeping in pace with the progress of fitness of high-risk patients. A delegate but all the more important consideration to 
make. Moreover, attention must be paid in the explanation to patients why screening, assessment, and prehabilitation are important, since the patient's overall thoughts often are - rather understandably with respect to their perceptions about the seriousness of their disease - that the tumor should be removed as soon as possible.

\section{Aim and outline of this thesis}

Although perioperative outcome of patients with colorectal cancer has improved during the last decades by the introduction of new surgical techniques and ERAS programs, ${ }^{24,67}$ preoperative risk assessment remains of high importance to further improve outcome and adapt patient-tailored treatment strategies (e.g., by selecting high-risk patients who may benefit from preoperative preventive (prehabilitation) interventions). Therefore, the aim of this thesis was to find tools to screen, assess, and diagnose patients with colorectal cancer at risk for postoperative complications throughout the preoperative period (with the focus on physical fitness in this period) and to investigate if the incidence of postoperative complications in especially the high-risk patients can be reduced with a community-based prehabilitation program.

- Chapter 2 describes prognostic factors for postoperative morbidity and tumor response after neoadjuvant chemoradiation followed by resection for rectal cancer.

- In chapter 3, the associations between skeletal muscle measurements (muscle mass, muscle radiation attenuation, and sarcopenic obesity) and postoperative outcome after treatment of locally advanced rectal cancer are evaluated.

- Chapter 4 aimed to investigate the validity of the oxygen uptake efficiency slope as an objective and submaximal indicator of aerobic fitness in elderly patients scheduled for major colorectal surgery.

- In chapter 5, the relationship between preoperative body composition and aerobic fitness of patients scheduled for colorectal surgery was evaluated.

- Chapter 6 describes the study protocol of a randomized controlled trial investigating the effects of prehabilitation versus usual care to reduce postoperative complications in high-risk patients with colorectal cancer or dysplasia scheduled for elective colorectal resection.

- In chapter 7 , the results of the randomized controlled trial described in chapter 6 are presented.

- Chapter 8 provides the general discussion, in which the main findings of the studies are summarized and discussed with reference to the current literature. Moreover, limitations, clinical relevance of the findings, and suggestions for future research are discussed.

- In chapter 9, a summary is provided.

- Finally, chapter 10 describes a reflection on the scientific impact of the results of the research described in this thesis, as well as the societal impact (impact-paragraph). 


\section{References}

1. Ministry of Health, Welfare and Sport (VWS). Available at: https://www.government.nl/ ministries/ministry-of-health-welfare-and-sport. Last accessed May 25, 2020.

2. World Health Organization. Men, Ageing and Health: achieving health across the life span. Available at: https://apps.who.int/iris/handle/10665/66941. Last accessed June 10, 2021.

3. Health Council of the Netherlands. Prevention in the elderly: Focus on functioning in daily life. The Hague: Health Council of the Netherlands, 2009; publication no. 2009/07. Available at: https://www.healthcouncil.nl/documents/advisory-reports/2009/04/21/prevention-in-theelderly-focus-on-functioning-in-daily-life. Last accessed May 25, 2020.

4. Ministry of Health, Welfare and Sport (VWS). National health policy note 2020-2024. Available at: https://www.rijksoverheid.nl/binaries/rijksoverheid/documenten/rapporten/2020/02/29/ gezondheid-breed-op-de-agenda/gezondheid-breed-op-de-agenda.pdf. Last accessed June 25, 2020.

5. Huber M, Knottnerus JA, Green L, et al. How should we define health? BMJ 2011; 343: d4163.

6. Huber M, van Vliet M, Giezenberg M, Winkens B, Heerkens Y, Dagnelie PC, Knottnerus JA. Towards a 'patient-centred' operationalisation of the new dynamic concept of health: a mixed methods study. BMJ Open 2016; 6(1): e010091,2015-010091.

7. Wynter-Blyth V, Moorthy K. Prehabilitation: preparing patients for surgery. BMJ 2017; 358: j3702.

8. Santa Mina D, Scheede-Bergdahl C, Gillis C, Carli F. Optimization of surgical outcomes with prehabilitation. Appl Physiol Nutr Metab 2015; 40(9): 966-9.

9. Hulzebos EH, van Meeteren NL. Making the elderly fit for surgery. Br J Surg 2016; 103(4): 463.

10. Prehabilitation, rehabilitation, and revocation in the Army. Br Med J 1946; 1: 192-7.

11. Silver JK, Baima J. Cancer prehabilitation: an opportunity to decrease treatment-related morbidity, increase cancer treatment options, and improve physical and psychological health outcomes. Am J Phys Med Rehabil 2013; 92(8): 715-27.

12. Agasi-Idenburg CS, Zuilen MK, Westerman MJ, Punt CJA, Aaronson NK, Stuiver MM. "I am busy surviving" - Views about physical exercise in older adults scheduled for colorectal cancer surgery. J Geriatr Oncol 2020; 11(3): 444-50.

13. Bray F, Ferlay J, Soerjomataram I, Siegel RL, Torre LA, Jemal A. Global cancer statistics 2018: GLOBOCAN estimates of incidence and mortality worldwide for 36 cancers in 185 countries. CA Cancer J Clin 2018; 68(6): 394-424.

14. Integraal Kankercentrum Nederland (IKNL). Nederlands Kanker Registratie cijfers. Incidentie darmkanker. April 16, 2019; Available at: https://www.iknl.nl/kankersoorten/darmkanker/ registratie/incidentie. Last accessed May 28, 2020.

15. Integraal Kankercentrum Nederland (IKNL). Nederlandse Kanker Registratie cijfers. Available at: https://www.iknl.nl/nkr-cijfers. Last accessed May 28, 2020. 
16. Dutch institute for clinical auditing (DICA) Leiden. DICA jaarrapportage 2018: Dutch ColoRectal Audit (DCRA). Available at: https://dica.nl/jaarrapportage-2018/dcra. Last accessed December 20, 2019.

17. Sloothaak DA, Geijsen DE, van Leersum NJ, et al. Optimal time interval between neoadjuvant chemoradiotherapy and surgery for rectal cancer. Br J Surg 2013; 100(7): 933-9.

18. van der Valk MJM, Hilling DE, Bastiaannet E, et al. Long-term outcomes of clinical complete responders after neoadjuvant treatment for rectal cancer in the International Watch \& Wait Database (IWWD): an international multicentre registry study. Lancet 2018; 391(10139): 253745.

19. Federatie Medisch Specialisten. Richtlijnendatabase. Colorectaal carcinoom. Last updated at October 29, 2019. Available at: https://richtlijnendatabase.nl/richtlijn/colorectaal_ carcinoom_crc/startpagina_-_crc.html. Last accessed June 18, 2020.

20. de Neree Tot Babberich MPM, van Groningen JT, Dekker E, et al. Laparoscopic conversion in colorectal cancer surgery; is there any improvement over time at a population level? Surg Endosc 2018; 32(7): 3234-46.

21. de Neree Tot Babberich MPM, Detering R, Dekker JWT, et al. Achievements in colorectal cancer care during 8 years of auditing in The Netherlands. Eur J Surg Oncol 2018; 44(9): 1361-70.

22. Gietelink L, Wouters MW, Bemelman WA, Dekker JW, Tollenaar RA, Tanis PJ, Dutch Surgical Colorectal Cancer Audit Group. Reduced 30-Day Mortality After Laparoscopic Colorectal Cancer Surgery: A Population Based Study From the Dutch Surgical Colorectal Audit (DSCA). Ann Surg 2016; 264(1): 135-40.

23. Oliphant R, Nicholson GA, Horgan PG, Molloy RG, McMillan DC, Morrison DS, West of Scotland Colorectal Cancer Managed Clinical Network. Contribution of surgical specialization to improved colorectal cancer survival. Br J Surg 2013; 100(10): 1388-95.

24. Ljungqvist O, Scott M, Fearon KC. Enhanced Recovery After Surgery: A Review. JAMA Surg 2017; 152(3): 292-8.

25. de Neree Tot Babberich MPM, Vermeer NCA, Wouters MWJM, et al. Postoperative Outcomes of Screen-Detected vs Non-Screen-Detected Colorectal Cancer in the Netherlands. JAMA Surg 2018; 153(12): e183567.

26. Heldens AFJM, Bongers BC, Lenssen AF, Stassen LPS, Buhre WF, van Meeteren NLU. The association between performance parameters of physical fitness and postoperative outcomes in patients undergoing colorectal surgery: An evaluation of care data. Eur J Surg Oncol 2017; 43(11): 2084-92.

27. Govaert JA, Fiocco M, van Dijk WA, et al. Costs of complications after colorectal cancer surgery in the Netherlands: Building the business case for hospitals. Eur J Surg Oncol 2015; 41(8): 1059-67.

28. Tevis SE, Kennedy GD. Postoperative Complications: Looking Forward to a Safer Future. Clin Colon Rectal Surg 2016; 29(3): 246-52. 
29. Moran J, Guinan E, McCormick P, et al. The ability of prehabilitation to influence postoperative outcome after intra-abdominal operation: A systematic review and meta-analysis. Surgery 2016; 160(5): 1189-201.

30. Walker KG, Bell SW, Rickard MJ, Mehanna D, Dent OF, Chapuis PH, Bokey EL. Anastomotic leakage is predictive of diminished survival after potentially curative resection for colorectal cancer. Ann Surg 2004; 240(2): 255-9.

31. Mirnezami A, Mirnezami R, Chandrakumaran K, Sasapu K, Sagar P, Finan P. Increased local recurrence and reduced survival from colorectal cancer following anastomotic leak: systematic review and meta-analysis. Ann Surg 2011; 253(5): 890-9.

32. Bates A, West MA, Jack S. Framework for prehabilitation services. Br J Surg 2020; 107(2): e114.

33. Dutch institute for clinical auditing (DICA) leiden. Factsheet indicatoren Dutch ColoRectal Audit (DCRA) 2019. Available at: https://dica.nl/dcra/documenten. Last accessed June 8, 2020.

34. Prado CM, Lieffers JR, McCargar LJ, Reiman T, Sawyer MB, Martin L, Baracos VE. Prevalence and clinical implications of sarcopenic obesity in patients with solid tumours of the respiratory and gastrointestinal tracts: a population-based study. Lancet Oncol 2008; 9(7): 629-35.

35. Rolland Y, Abellan van Kan G, Gillette-Guyonnet S, Vellas B. Cachexia versus sarcopenia. Curr Opin Clin Nutr Metab Care 2011; 14(1): 15-21.

36. Lieffers JR, Bathe OF, Fassbender K, Winget M, Baracos VE. Sarcopenia is associated with postoperative infection and delayed recovery from colorectal cancer resection surgery. $\mathrm{Br} J$ Cancer 2012; 107(6): 931-6.

37. Reisinger KW, van Vugt JL, Tegels JJ, et al. Functional compromise reflected by sarcopenia, frailty, and nutritional depletion predicts adverse postoperative outcome after colorectal cancer surgery. Ann Surg 2015; 261(2): 345-52.

38. Miyamoto $\mathrm{Y}$, Baba $\mathrm{Y}$, Sakamoto $\mathrm{Y}$, et al. Sarcopenia is a Negative Prognostic Factor After Curative Resection of Colorectal Cancer. Ann Surg Oncol 2015; 22(8): 2663-8.

39. Fearon K, Strasser F, Anker SD, et al. Definition and classification of cancer cachexia: an international consensus. Lancet Oncol 2011; 12(5): 489-95.

40. van Vugt JL, Levolger S, Gharbharan A, et al. A comparative study of software programmes for cross-sectional skeletal muscle and adipose tissue measurements on abdominal computed tomography scans of rectal cancer patients. J Cachexia Sarcopenia Muscle 2017; 8(2): 285-97.

41. Prado CM, Birdsell LA, Baracos VE. The emerging role of computerized tomography in assessing cancer cachexia. Curr Opin Support Palliat Care 2009; 3(4): 269-75.

42. Martin L, Birdsell L, Macdonald N, et al. Cancer cachexia in the age of obesity: skeletal muscle depletion is a powerful prognostic factor, independent of body mass index. J Clin Oncol 2013; 31(12): 1539-47.

43. Boer BC, de Graaff F, Brusse-Keizer M, Bouman DE, Slump CH, Slee-Valentijn M, Klaase JM. Skeletal muscle mass and quality as risk factors for postoperative outcome after open colon resection for cancer. Int J Colorectal Dis 2016; Jun;31(6): 1117-24. 
44. Sabel MS, Terjimanian M, Conlon AS, et al. Analytic morphometric assessment of patients undergoing colectomy for colon cancer. J Surg Oncol 2013; 108(3): 169-75.

45. Moran J, Wilson F, Guinan E, McCormick P, Hussey J, Moriarty J. Role of cardiopulmonary exercise testing as a risk-assessment method in patients undergoing intra-abdominal surgery: a systematic review. Br J Anaesth 2016; 116(2): 177-91.

46. West MA, Asher R, Browning M, et al. Validation of preoperative cardiopulmonary exercise testing-derived variables to predict in-hospital morbidity after major colorectal surgery. $\mathrm{Br} \mathrm{J}$ Surg 2016; 103(6): 744-52.

47. Davies LC, Wensel R, Georgiadou P, Cicoira M, Coats AJ, Piepoli MF, Francis DP. Enhanced prognostic value from cardiopulmonary exercise testing in chronic heart failure by non-linear analysis: oxygen uptake efficiency slope. Eur Heart J 2006; 27 (6): 684-90.

48. Myers J, Arena R, Dewey F, et al. A cardiopulmonary exercise testing score for predicting outcomes in patients with heart failure. Am Heart J 2008; 156(6): 1177-83.

49. Coeckelberghs E, Buys R, Goetschalckx K, Cornelissen VA, Vanhees L. Prognostic value of the oxygen uptake efficiency slope and other exercise variables in patients with coronary artery disease. Eur J Prev Cardiol 2016; 23(3): 237-44.

50. Lin YS, Huang HY, Lin WH, et al. Oxygen Uptake Efficiency Slope Predicts Major Cardiac Events in Patients With End-Stage Heart Failure. Transplant Proc 2016; 48(3): 956-8.

51. Boo SH, Joo MC, Lee JM, Kim SC, Yu YM, Kim MS. Association between skeletal muscle mass and cardiorespiratory fitness in community-dwelling elderly men. Aging Clin Exp Res 2019; 31(1): 49-57.

52. Bongers BC, Punt IM, van Meeteren NL. On "Prehabilitation: The emperor's new clothes or a new arena for physical therapists?" Lundberg M, Archer KR, Larsson C, Rydwik E. Phys Ther. 2018;12:127-130. Phys Ther 2019; 99(7): 953-4.

53. Thomas G, Tahir MR, Bongers BC, Kallen VL, Slooter GD, van Meeteren NL. Prehabilitation before major intra-abdominal cancer surgery: A systematic review of randomised controlled trials. Eur J Anaesthesiol 2019; 36(12): 933-45.

54. Bruns ER, van den Heuvel B, Buskens $C J$, et al. The effects of physical prehabilitation in elderly patients undergoing colorectal surgery: a systematic review. Colorectal Dis 2016; 18(8): O26777.

55. Hijazi Y, Gondal U, Aziz O. A systematic review of prehabilitation programs in abdominal cancer surgery. Int J Surg 2017; 39: 156-62.

56. Carli F, Bousquet-Dion G, Awasthi R, et al. Effect of Multimodal Prehabilitation vs Postoperative Rehabilitation on 30-Day Postoperative Complications for Frail Patients Undergoing Resection of Colorectal Cancer: A Randomized Clinical Trial. JAMA Surg 2020; 155(3): 233-42.

57. Hulzebos EH, Helders PJ, Favie NJ, De Bie RA, Brutel de la Riviere A, Van Meeteren NL. Preoperative intensive inspiratory muscle training to prevent postoperative pulmonary complications in high-risk patients undergoing CABG surgery: a randomized clinical trial. JAMA 2006; 296(15): 1851-7. 
58. Topp R, Ditmyer M, King K, Doherty K, Hornyak J,3rd. The effect of bed rest and potential of prehabilitation on patients in the intensive care unit. AACN Clin Issues 2002; 13(2): 263-76.

59. Hoogeboom TJ, Oosting E, Vriezekolk JE, et al. Therapeutic validity and effectiveness of preoperative exercise on functional recovery after joint replacement: a systematic review and meta-analysis. PLoS One 2012; 7(5): e38031.

60. Barberan-Garcia A, Ubre M, Roca J, et al. Personalized Prehabilitation in High-risk Patients Undergoing Elective Major Abdominal Surgery: A Randomized Blinded Controlled Trial. Ann Surg 2018; 267(1): 50-6.

61. Bongers BC, Klaase JM, van Meeteren NLU. Prehabilitation vs Postoperative Rehabilitation for Frail Patients. JAMA Surg 2020; doi: 10.1001/jamasurg.2020.1801.

62. Glasziou P, Irwig L, Mant D. Monitoring in chronic disease: a rational approach. BMJ 2005; 330(7492): 644-8.

63. van Buuren S, Hulzebos EH, Valkenet K, Lindeman E, van Meeteren NL. Reference chart of inspiratory muscle strength: a new tool to monitor the effect of preoperative training. Physiotherapy 2014; 100(2): 128-33.

64. Stichting Oncologische Samenwerking (SONCOS). Multidisciplinaire normering oncologische zorg in Nederland, normeringsrapport 2018. Available at: https://www.soncos.org/wpcontent/uploads/2018/02/Soncos_norm-rapp2018.pdf. Last accessed August 7, 2020.

65. Weston M, Weston KL, Prentis JM, Snowden CP. High-intensity interval training (HIT) for effective and time-efficient pre-surgical exercise interventions. Perioper Med (Lond) 2016; 5: 2,015-0026-8. eCollection 2016.

66. Bongers BC, Dejong CHC, den Dulk M. Enhanced recovery after surgery programmes in older patients undergoing hepatopancreatobiliary surgery: what benefits might prehabilitation have? Eur J Surg Oncol 2020; https://doi.org/10.1016/j.ejso.2020.03.211.

67. van Vugt JL, Reisinger KW, Derikx JP, Boerma D, Stoot JH. Improving the outcomes in oncological colorectal surgery. World J Gastroenterol 2014; 20(35): 12445-57. 



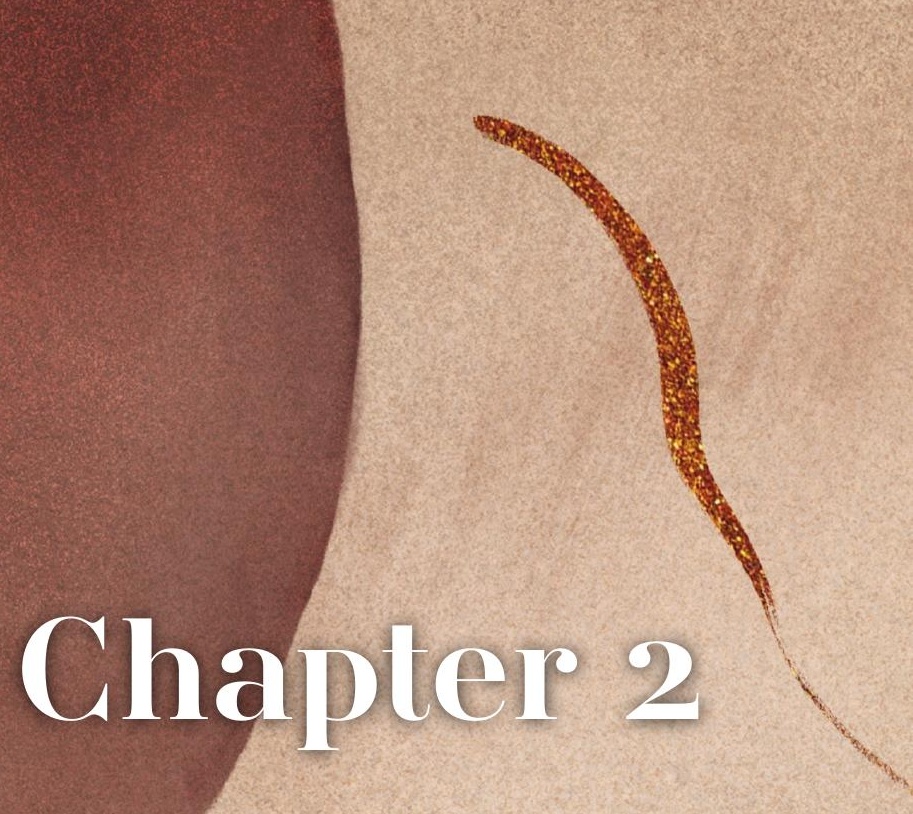

Prognostic factors for postoperative morbidity and tumor response after neoadjuvant chemoradiation followed by resection for rectal cancer

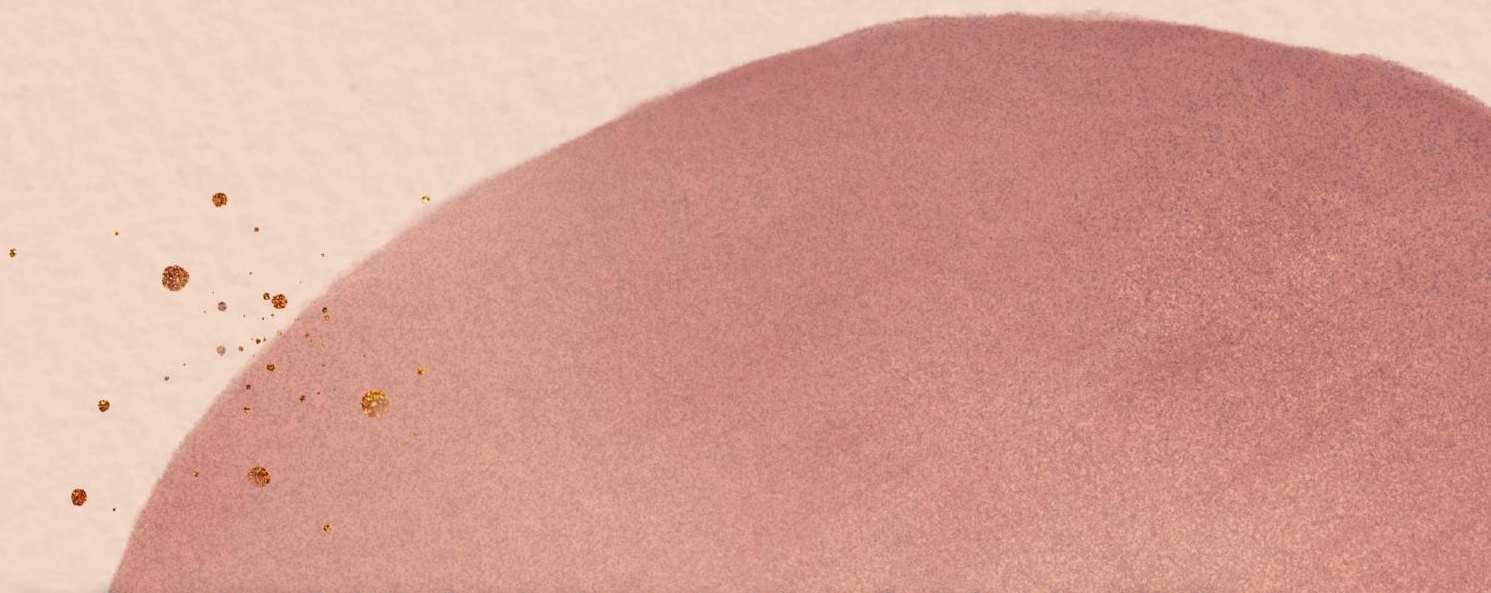

Annefleur E. M. Berkel, Dankert P. Woutersen, Job van der Palen, Joost M. Klaase 


\section{Abstract}

Background: In patients with locally advanced rectal cancer treated with neoadjuvant chemoradiation followed by rectal resection, postoperative morbidity is a significant clinical problem. Pathologic complete tumor response seems to give the best prognosis in the long term. Little is known about the factors that are associated with postoperative complications and pathologic complete response. The aim of this retrospective study was to identify and describe these factors.

Methods: Ninety-nine consecutive patients with locally advanced rectal cancer who underwent neoadjuvant chemoradiation (50 Gy and capecitabine) followed by surgery at our institute between January 2007 and May 2012 were identified. Postoperative complications were graded according to the Clavien-Dindo classification. Pathologic tumor response was categorized as complete response or no/partial response.

Results: Postoperative complications occurred in 68 patients (69\%) and grade 3-5 complications in 25 patients (25\%). The 30-day and 90-day mortality were $1 \%(n=1)$ and $2 \%(n=2)$, respectively. A young age $(p=0.021)$ and a preoperative or postoperative blood transfusion $(p=0.015)$ independently predicted complications. Intraoperative or postoperative blood transfusion ( $p=0.007)$ and ypT0-1 stage $(p=0.037)$ were independent predictors for grade $3-5$ complications. Complete response rate was $22 \%(n=22) ; 4 \%(n=4)$ of patients showed no response. No independent factors predicting complete response were found.

Conclusions: Neoadjuvant chemoradiation followed by rectal resection is associated with significant postoperative morbidity but minimal postoperative mortality. A complete response rate of $22 \%$ was achieved. 


\section{Introduction}

\section{Postoperative complications}

In our hospital $43 \%$ of patients with colorectal cancer has a locally advanced carcinoma. ${ }^{1}$ According to the Dutch guidelines, treatment of locally advanced rectal cancer consists of neoadjuvant chemoradiation followed by rectal surgery. ${ }^{2}$ This extensive treatment regimen often results in postoperative complications. The postoperative complication rate is about $40 \%{ }^{3,4}$ Especially anastomotic leakage following low anterior resection (LAR) and perineal wound complications following abdominoperineal resection (APR) are important and frequently observed complications, with an average of $10 \%{ }^{5}$ and $35 \%$, respectively. ${ }^{6,7}$ Kerr et al. (2008) ${ }^{6}$ showed that a shorter interval between chemoradiation and rectal surgery significantly predicted more anastomotic leakages and perineal wound complications. Stelzmueller et al. (2009) ${ }^{4}$ showed that women, low pre-therapeutic hemoglobin level, and higher American Society of Anesthesiologists (ASA) score were associated with early postoperative complications. Also, elderly patients are prone to complications, especially general complications, because of their comorbidities and the physiologic factors that play a role in aging. ${ }^{5}$ These complications often lead to a prolonged hospitalization and also to a higher mortality rate. ${ }^{5}$ Identifying factors associated with postoperative complications seems important, because postoperative complications are associated with worse long-term survival rates. ${ }^{4}$

\section{Tumor response after chemoradiation}

Neoadjuvant chemoradiation for locally advanced rectal cancer has been shown to achieve pathologic tumor downstaging, with improvement of resectability and sphincter saving surgery, ${ }^{8}$ and has been shown to reduce the risk of developing local recurrences or distance recurrences. ${ }^{9}$ Pathologic complete response ( $\mathrm{pCR}$ ) seems to translate into improved clinical results. There is a large variability in response to neoadjuvant chemoradiation between patients. Some patients do not respond at all and may even have progression of their disease, while others have a pCR. Incidence of $\mathrm{pCR}$ ranges from $10 \%$ to $24 \%$. $^{3,4,6,10-13}$

Several studies showed that a longer interval between chemoradiation and surgery significantly improves the rate of $\mathrm{pCR} .{ }^{3,10,11,14-16}$ It remains unknown what the optimal time interval between chemoradiation and surgery is. Increased intervals could potentially increase the tumor downstaging effect because radiation-induced necrosis appears to be a time-dependent phenomenon, but a longer interval can also allow the tumor to progress and decrease survival. ${ }^{17}$ Das et al. $(2008)^{12}$ showed that a circumferential extent of the tumor $>60 \%$, carcinoembryonic antigen (CEA) level $>2.5 \mathrm{ng} / \mathrm{ml}$, and tumor distance from the anal verge $>5 \mathrm{~cm}$ were associated significantly with a lower $p C R$ rate. 
Identifying factors that are associated with pCR could help clinicians with treatment decisions and determining prognosis. Few studies investigated these factors, so it is unknown which patients with locally advanced rectal cancer benefit the most from treatment with neoadjuvant chemoradiation.

Aim of the present study was to identify prognostic factors for postoperative complications and $\mathrm{PCR}$ in patients with locally advanced rectal cancer treated with neoadjuvant chemoradiation followed by surgery.

\section{Materials and methods}

All consecutive patients between January 2007 and May 2012 with locally advanced rectal cancer who had neoadjuvant chemoradiation and surgery in Medical Spectrum Twente, a large non-academic teaching hospital in the Netherlands, were retrospectively identified. Locally advanced rectal cancer was defined as a clinical T4 tumor or T3 tumor with a threatened circumferential margin (CRM $<5 \mathrm{~mm}$ ) and/or clinical lymph node stage 2 (cN2) disease. Colonoscopy with biopsy was performed to histologically confirm the diagnosis. Magnetic resonance imaging (MRI) was used to evaluate tumor infiltration and the presence of lymph nodes larger than $5 \mathrm{~mm}$ or with characteristics suspicious of metastases. To evaluate dissemination, a CT-abdomen and an X-ray or CT-thorax was used.

Patients underwent a standard protocol of concomitant chemotherapy and threedimensional conformal radiotherapy (3D-CRT). Preoperative radiotherapy consisted of $50 \mathrm{~Gy}$ in 25 fractions during five weeks. Clinical target volume (CTV) was defined as mesorectum, plus lateral nodes up to bifurcation from vena iliaca interna and externa plus presacral nodes. Anal complex was only part of the CTV in distal tumors (CTV at least $3 \mathrm{~cm}$ caudal from gross tumor volume). Treatment planning according to International Commission on Radiation Units and Measurements (ICRU) 50; 3D-CRT was performed with 3-5 18 megavoltage (MV) photon beams, but from January 2012 intensity modulated radiotherapy (IMRT)/volumetric modulated arc therapy (VMAT) $6 \mathrm{MV}$. The planning system was Varian Eclipse version 6.7 and 8.9. Position verification was done on bony anatomy with two orthogonal images. In the early years an offline-MV setup correction-protocol was used, and from 2009 on an online-kilovoltage $(\mathrm{kV})$ correction-protocol was used. Chemotherapy consisted of $825 \mathrm{mg} / \mathrm{m}^{2}$ capecitabine administered two times daily on days of radiotherapy and also over weekends. All operations were carried out according to the total mesorectal excision (TME) principle and consisted of an APR, LAR, or a Hartmann procedure. Between 2007 and 2010, an interval between chemoradiation and surgery of 6-8 weeks was considered optimal; between 2010 and 2011, this was 8-10 weeks, and since 2012, an interval of 10-12 weeks was considered optimal. Actual intervals varied as 
a result of logistical factors, patient morbidity and surgeons' and patients' scheduling preferences.

The examined variables included patient and tumor characteristics, neoadjuvant chemoradiation regimen, symptoms of obstruction requiring fecal diversion before rectal surgery, interval between the end of chemoradiation and resection, complications of chemoradiation, type of surgery, diverting stoma (only for patients undergoing LAR), operation time, estimated blood loss, blood transfusion intra- or postoperative, pretreatment tumor distance from the anal verge, hospitalization, postoperative surgical and general complications, 30-day and 90-day mortality, pathologic tumor response, pathologic TNM stage, completeness of surgical excision ( $R$ stage), CEA level before neoadjuvant chemoradiation, and preoperative hemoglobin $(\mathrm{Hb})$. Age was divided in four groups, in which group 1 represent the youngest patients and group 4 the oldest.

Postoperative surgical complications until six months after surgery were scored using prespecified definitions (Table 1). The severity was scored using the Clavien-Dindo classification of surgical complications (grade 1-5). ${ }^{18,19}$ Grade 1 complications are complications without the need for treatment (except medication as antiemetics, and wound infections opened at bedside). Grade 2 complications require pharmacological treatment. Grade 3 complications require surgical, endoscopic, or radiological intervention. Grade 4 complications are life-threatening complications requiring intensive care/intensive care unit (IC/ICU) management. Grade 5 is death of a patient. General complications were scored as cardiovascular, pulmonary, renal, or neurological. Pathologic tumor response was divided into complete response and no/partial response. Pathologic complete response was defined as absence of viable adenocarcinoma cells in the surgical specimen, including primary tumor and lymph nodes. Patients were dichotomized into two groups according to the interval between neoadjuvant chemoradiation and surgery, following a proposal published in the literature: $\leq 7$ weeks (short-interval group) and $>7$ weeks (long-interval group). .,11 $^{31}$

\section{Statistical analysis}

Data was analyzed with Statistical Package for the Social Sciences (SPSS) version 20.0. Data are presented with median and range or mean and standard deviation, as appropriate. Categorical data are summarized by frequency and percentage within each cohort. The univariate associations between variables and postoperative (grade 3-5) complications and pathologic complete response were evaluated for significance, using the Fisher exact test or $\mathrm{Chi}^{2}$ test for categorical variables and the Mann Whitney $\mathrm{U}$ test or independent $t$-test (if normally distributed) for continuous variables. If the number of persons per category was small, categories were merged. Variables with a $p<0.150$ in univariate analysis have been included in a multivariate logistic regression analysis to identify independent 
predictors of postoperative (grade 3-5) complications and pathologic complete response. A $p<0.050$ was considered statistically significant.

Table 1 Definition of surgical complications (within six months after surgery).

\begin{tabular}{|c|c|}
\hline Complication & Definition \\
\hline Relaparotomy & Any unplanned relaparotomy \\
\hline Anastomotic leakage & $\begin{array}{l}\text { Any fluid collection around the anastomosis following LAR: clinical } \\
\text { suspicion confirmed by surgery or diagnosed on CT-scan }\end{array}$ \\
\hline $\begin{array}{l}\text { Perineal wound } \\
\text { complication }\end{array}$ & $\begin{array}{l}\text { Wound dehiscence, wound infections, wound necrosis, abscess or } \\
\text { delayed wound healing ( }>6 \text { weeks persisting skin defect) following APR }\end{array}$ \\
\hline Rectal stump abscess & $\begin{array}{l}\text { Any fluid collection around the rectal stump/presacral after a } \\
\text { Hartmann procedure }\end{array}$ \\
\hline $\begin{array}{l}\text { Abdominal wound } \\
\text { complication }\end{array}$ & $\begin{array}{l}\text { Wound dehiscence, wound infections, wound necrosis, abscess or } \\
\text { delayed wound healing ( }>6 \text { weeks persisting skin defect) }\end{array}$ \\
\hline Intra-abdominal abscess & $\begin{array}{l}\text { Any intra-abdominal fluid collection unrelated to the anastomosis or } \\
\text { perineal wound }\end{array}$ \\
\hline Fistula & $\begin{array}{l}\text { Enterocutaneous, enterovaginal, vesicovaginal or enterovesical } \\
\text { connections }\end{array}$ \\
\hline Sepsis & Systemic inflammatory response syndrome caused by an infection \\
\hline Ileus & $\begin{array}{l}\text { Absence of bowel sounds or defecation more than five days following } \\
\text { surgery; need for placement or delayed removal of nasogastric tube }\end{array}$ \\
\hline Bleeding & $\begin{array}{l}\text { Gastrointestinal hemorrhage, decrease in hemoglobin level directly } \\
\text { after surgery treated conservatively with or without blood transfusions } \\
\text { or by reintervention }\end{array}$ \\
\hline Intestinal necrosis & Any bowel ischemia \\
\hline Stoma complication & Problems with stoma, such as parastomal hernia or stenosis \\
\hline Urological & $\begin{array}{l}\text { Ureter leakage, urinary incontinence, ureter stenosis, suprapubic } \\
\text { or transurethral catheter-related problems, urinary tract infections, } \\
\text { urinary retention, urosepsis }\end{array}$ \\
\hline Sexual dysfunction & $\begin{array}{l}\text { Sexual problem such as retrograde ejaculation, secondary } \\
\text { dyspareunia }\end{array}$ \\
\hline
\end{tabular}

Abbreviations: APR, abdominoperineal resection; CT, computed tomography; Hb, hemoglobin; LAR, low anterior resection.

\section{Results}

A total of 105 patients with locally advanced rectal cancer underwent neoadjuvant chemoradiation. Of these 105 patients, 103 underwent laparotomy. Two patients were excluded preoperative, one because of new metastases and one because of comorbidity. At laparotomy, resection was possible in 99 patients, with a median age of 66 (range 40-81) years. In four patients, the tumor process was unresectable (Figure 1). Patient, tumor, and treatment characteristics are shown in Table 2. 
Ninety-six patients (97\%) received all planned 25 fractions of 2 Gy radiotherapy. Two patients received $42 \mathrm{~Gy}$, one patient because of a rectovaginal fistula and one patient because of a very poor clinical condition. One patient received $48 \mathrm{~Gy}$ at his own request. Capecitabine intake was stopped prematurely in fifteen patients (15\%) because of complications: diarrhea (5), rectovaginal fistula (1), neutropenic colitis (1), hand-foot syndrome (3), nausea (3), fatigue (1), atrial fibrillation (2), acute coronary syndrome (1), leukopenia (1), anaemia (1), and rash (1).

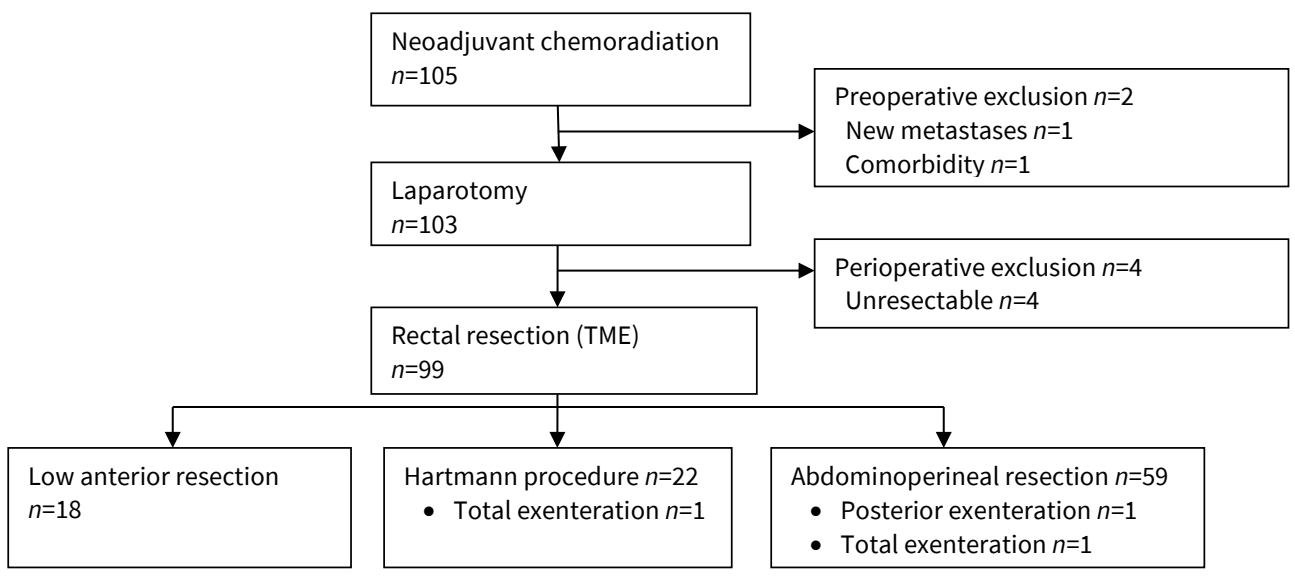

Figure 1 Flow of treatment with number of patients $(n)$ and reasons of exclusion. Abbreviation: TME, total mesorectal excision.

Median interval between chemoradiation and surgery was 7.9 (range 3.9-34.4) weeks; 41 patients $(41.4 \%)$ had an interval of $\leq 7$ weeks and 58 patients $(58.6 \%)$ had an interval of $>7$ weeks. One patient had an interval of 34.4 weeks because of an ischemic cerebrovascular accident in the period between chemoradiation and surgery. Fifty-nine patients underwent APR (59.6\%), 22 patients Hartmann (22.2\%), and 18 patients LAR (18.2\%). Sixteen patients $(88.9 \%)$ had a diverting stoma after LAR. Several months after LAR, restoration of intestinal continuity was achieved in fifteen patients (94\%). In 85 patients (85.9\%) the surgical resection was complete, with no residual tumor left (R0 stage), and in 14 patients (14.1\%) there was microscopically residual tumor (CRM <1 mm; R1 stage). Mean operation time was 142 (SD 59) minutes and mean estimated blood loss was 400 (range 0-2000) ml.

\section{Postoperative complications}

Overall postoperative morbidity rate in 99 patients was $68.7 \%(n=68)$. In 43 patients $(43.4 \%)$, this was a grade $1-2$ complication, and in 25 patients $(25.3 \%)$, this was a grade 3-5 complication. Of these patients, 44 had more than one postoperative complication. Postoperative complications are summarized in Table 3. A perineal wound complication was seen in 30 of 59 patients (50.8\%) who underwent APR, mostly grades 1-2. Anastomotic 
leakage developed in 2 of 18 patients undergoing LAR. Twenty-one patients had a general complication. Twenty of 99 patients $(20.2 \%)$ required surgical reintervention. Thirty-nine of 99 patients (39.4\%) had an intraoperative or postoperative blood transfusion. One patient (1\%) died within 30 days after surgery, as a result of a sepsis of unknown cause. Another patient died 67 days after surgery as a result of advanced metastatic disease, making the 90 -day mortality rate $2 \%$. Median hospital stay was 10 (range 3-131) days.

Table 2 Patient, tumor and treatment characteristics.

\begin{tabular}{|c|c|}
\hline & No. of patients $(n=99)^{a}$ \\
\hline \multicolumn{2}{|l|}{ Age } \\
\hline Group 1 (40-58 years) & $26(26.3)$ \\
\hline Group 2 (59-65 years) & $22(22.2)$ \\
\hline Group 3 (66-70 years) & $26(26.3)$ \\
\hline Group 4 (71-81 years) & $25(25.3)$ \\
\hline \multicolumn{2}{|l|}{ Gender } \\
\hline Male & $53(53.5)$ \\
\hline Female & $46(46.5)$ \\
\hline \multicolumn{2}{|l|}{$\mathrm{BMI}\left(\mathrm{kg} / \mathrm{m}^{2}\right)$} \\
\hline$<25$ & $32(32.3)$ \\
\hline $25-29.9$ & $47(47.5)$ \\
\hline$\geq 30$ & $17(17.2)$ \\
\hline Unknown & $3(3.0)$ \\
\hline \multicolumn{2}{|l|}{ ASA } \\
\hline 1 & $20(20.2)$ \\
\hline II & $70(70.7)$ \\
\hline III & $9(9.1)$ \\
\hline IV-V & $0(0)$ \\
\hline \multicolumn{2}{|l|}{ Charlson score } \\
\hline 0 & $67(67.7)$ \\
\hline 1 & $16(16.2)$ \\
\hline$\geq 2$ & $16(16.2)$ \\
\hline \multicolumn{2}{|l|}{ Clinical T stage } \\
\hline cT2 & $3(3.0)$ \\
\hline cT3 & $63(63.6)$ \\
\hline cT4 & $33(33.3)$ \\
\hline \multicolumn{2}{|l|}{ Clinical N stage } \\
\hline $\mathrm{cNO}$ & $10(10.1)$ \\
\hline $\mathrm{cN} 1$ & $44(44.4)$ \\
\hline $\mathrm{cN} 2$ & $44(44.4)$ \\
\hline $\mathrm{cNx}$ & $1(1.0)$ \\
\hline \multicolumn{2}{|l|}{ Clinical M stage } \\
\hline cM0 & $93(93.9)$ \\
\hline cM1 & $6(6.1)$ \\
\hline
\end{tabular}


Distance from anal verge $(\mathrm{cm})$

$<5$

5-10

$>10$

Unknown

Stoma before rectal surgery

Yes

No

Pathological T stage

урт0

урT1

урт2

урт3

урT4

Pathological N stage

ypNO

ypN1

ypN2

ypNx

Tumor response

No

Partial

Complete

Unknown
41 (41.4)

$52(52.5)$

$5(5.1)$

$1(1.0)$

$12(12.1)$

87 (87.9)

$23(23.2)$

10 (10.1)

18 (18.2)

41 (41.4)

7 (7.1)

68 (68.7)

22 (22.2)

8 (8.1)

1 (1.0)

4 (4.0)

72 (72.7)

22 (22.2)

1 (1.0)

a: With percentages in parentheses.

Abbreviations: ASA, American Society of Anesthesiologists score; BMI, body mass index. 


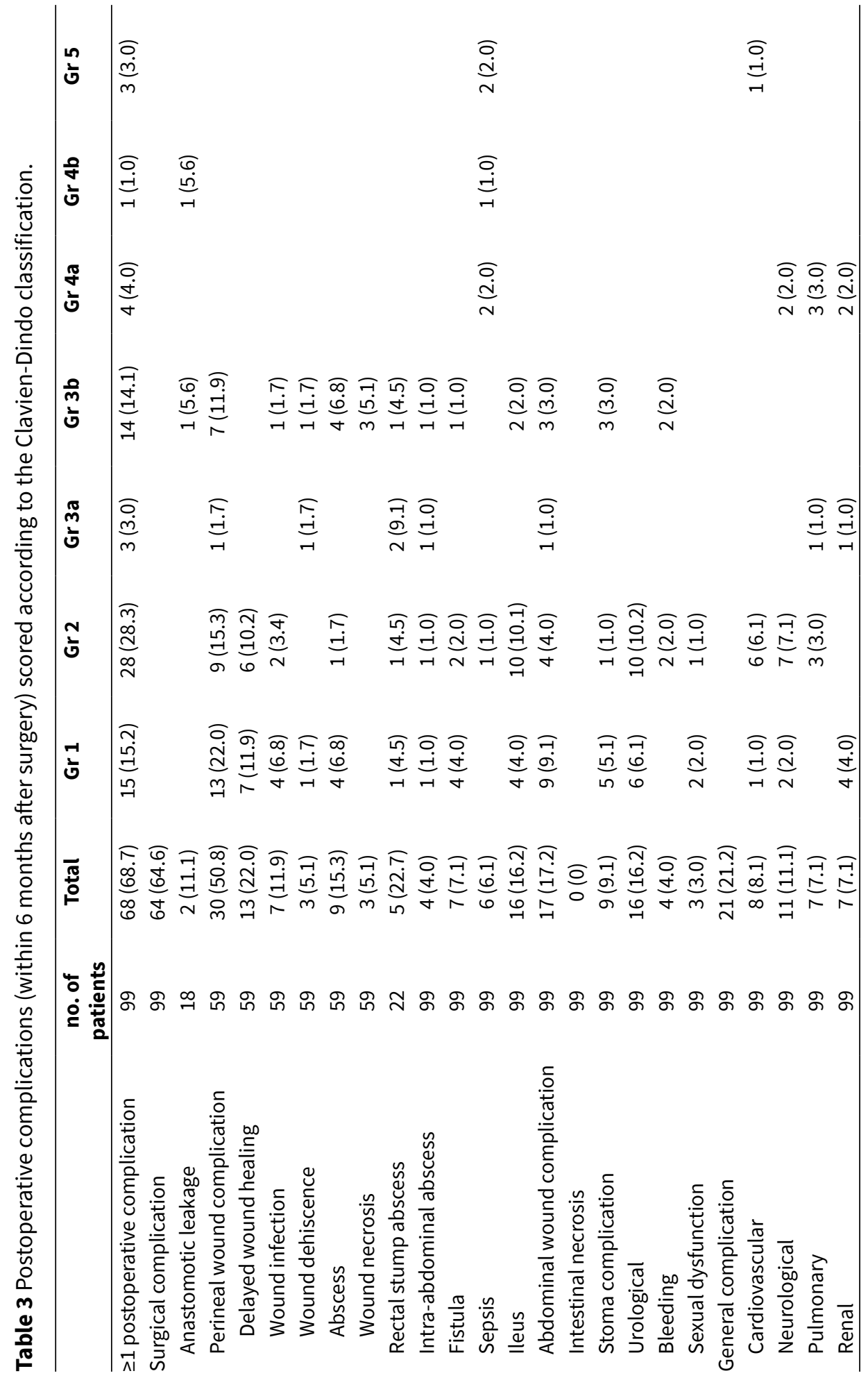


Table 4 displays the univariate results between variables and postoperative (grade 3-5) complications (shown if $p<0.150$ ). The youngest patient group developed more often a postoperative complication than the older patient groups $(p=0.017)$. Men significantly had more often a grade $3-5$ complication than women ( $34 \%$ versus $15 \% ; p=0.032$ ). Postoperative (grade 3-5) complications were significantly associated with intra- or postoperative blood transfusions. Complication rate was not significantly associated with interval between chemoradiation and surgery.

Variables with a $p<0.150$ in univariate analysis (age, operation time, blood transfusion) were included in the multivariate analysis to predict postoperative complications. Operation time was removed from the equation because it did not significantly predicted complications. Young age (odds ratio (OR) 7.51; 95\% confidence interval $(\mathrm{Cl})=1.35-41.88$; $p=0.021$ ) and an intra- or postoperative blood transfusion (OR 3.57; 95\% Cl=1.29-9.94; $p=0.015$ ) were independent predictors of postoperative complications.

Another multivariate analysis was done to predict postoperative grade 3-5 complications. Gender, Charlson score, CEA before chemoradiation, ypT stage, and blood transfusion were included in the multivariate analysis. An intraoperative or postoperative blood transfusion (OR 4.19; 95\% Cl=1.49-11.77; $p=0.007$ ) and an ypT0-1 (OR 3.19; 95\% Cl=1.079.50; $p=0.037$ ) independently predicted grade $3-5$ complications.

\section{Tumor response after chemoradiation}

Twenty-two of 99 patients (22\%) had a pCR after neoadjuvant chemoradiation. There were no significant variables associated with $\mathrm{PCR}$. A lower Charlson score $(p=0.058)$ and a lower CEA level before neoadjuvant chemoradiation $(p=0.053)$ seem to be associated with a higher $\mathrm{PCR}$ rate, but only reached borderline significance. Pathologic complete tumor response occurred in $24.6 \%$ of patients with an interval $>7$ weeks between chemoradiation and surgery and in $19.5 \%$ of patients with an interval $\leq 7$ weeks $(p=0.555)$. Multivariate logistic regression analysis indicated no significant independent predictors of $\mathrm{pCR}$. 


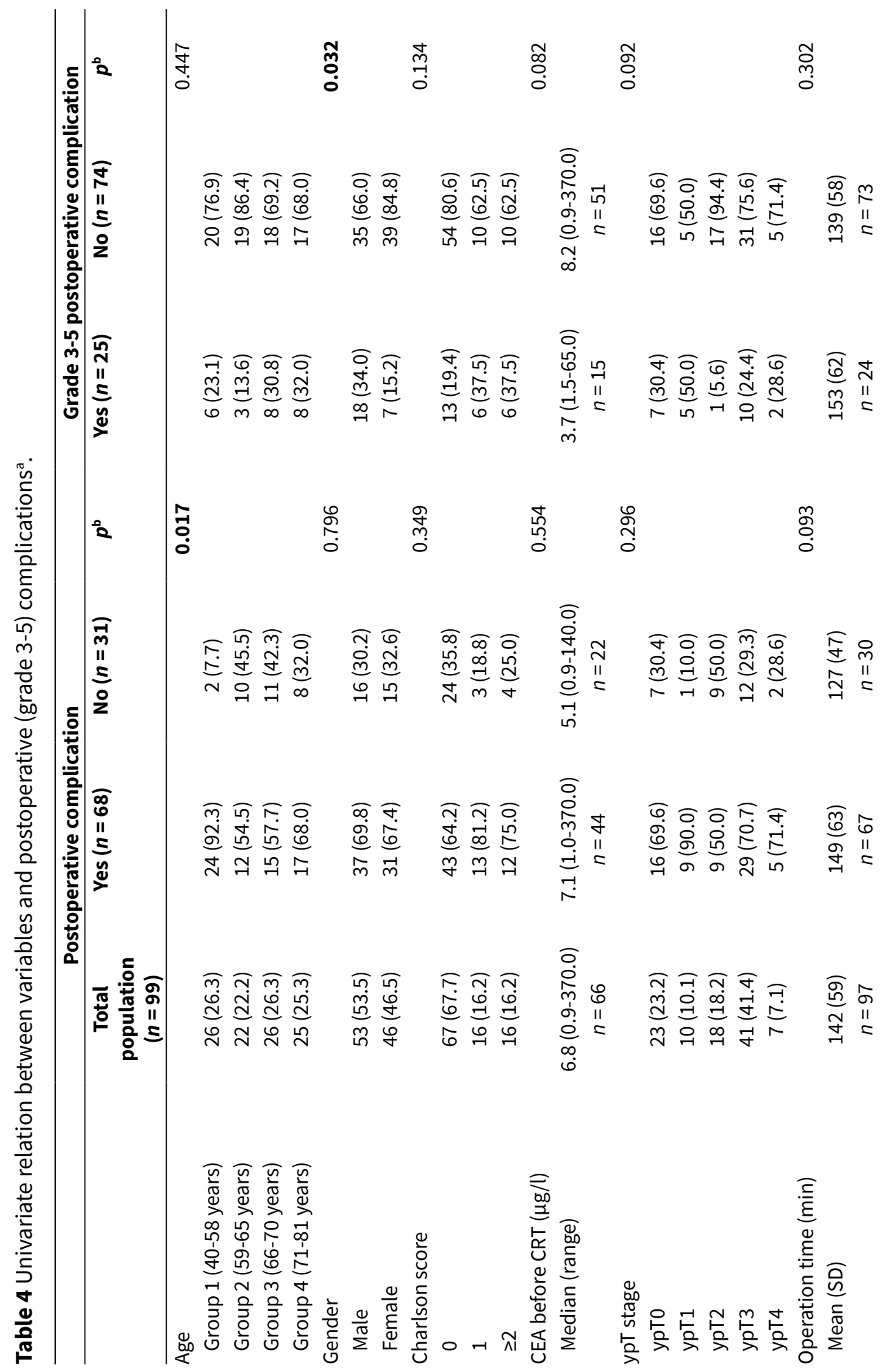




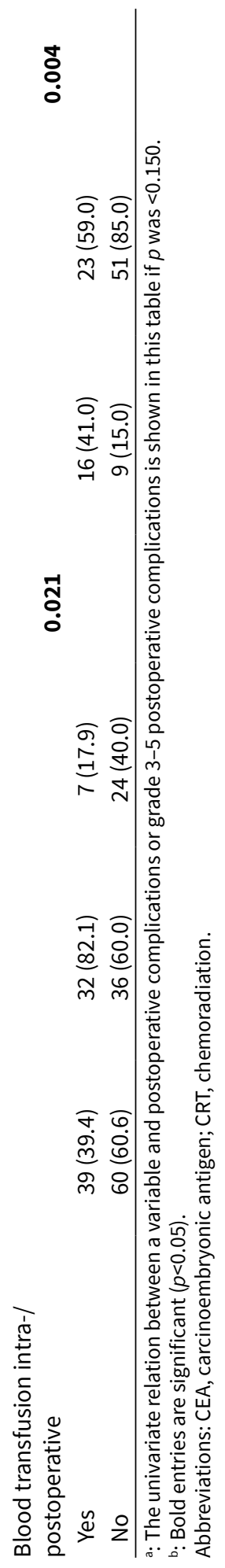




\section{Discussion}

This retrospective study found that postoperative complication rate was significant at $69 \%$, but this did not translate into postoperative mortality. Age, intra- or postoperative blood transfusions, and ypT stage predicted postoperative complications and/or postoperative grade 3-5 complications. Pathologic complete response rate was $22 \%$. However, no clinical prognostic factors could be found.

The postoperative complication rate in this study was higher than the rates of $15-56 \%$ reported in literature. ${ }^{3,4,7,11,13,14,16,20,21}$ A plausible reason for this difference could be the length of follow-up (six months) to describe postoperative complications in this study, whereas other studies described the 30-day postoperative complication rate., $4,7,13,21$ Swellengrebel et al. (2011) describes that a perineal wound may seem to heal normally initially, but subsequently manifest with a complication after discharge from hospital. This underlies the importance of a longer follow-up to describe postoperative complications. Another explanation could be that this study investigated many different and predefined complications, whereas other studies did not describe their definition of postoperative complications ${ }^{14,20,22}$ or only looked at a few complications as a measure of postoperative morbidity. ${ }^{6,11}$

Anastomotic leakage was diagnosed in 2 of 18 patients undergoing LAR. This is in line with rates of $6-28 \%$ reported in literature after neoadjuvant therapy. ${ }^{3,4,6,7,11,13-15} \mathrm{~A}$ perineal wound complication was observed in $51 \%$ of patients following APR. Other investigators reported percentages of $0-47 \%, 3,4,7,13,15,23$ In the present study perineal wound complications are defined as delayed wound healing (>6 weeks), wound infection, wound dehiscence, abscess, or wound necrosis within six months after APR, as suggested by Wiatrek et al. (2008). ${ }^{24}$ Some other studies only investigated one or two of these complications. ${ }^{3,4,6,15}$ Bullard et al. (2005) $)^{23}$ evaluated perineal wound complications after APR in 160 patients; 117 patients received preoperative radiation therapy. Overall perineal wound complication rate in patients who received preoperative radiation therapy was $47 \%$, and in patients who did not receive preoperative radiation therapy this was $23 \%$. The risk of a perineal wound complication more than doubled with the addition of preoperative radiation therapy. ${ }^{23}$ Pelvic irradiation induces cell death and progressive occlusive vasculitis that affects not only the tumor, but also surrounding healthy tissues. ${ }^{23}$ This damage may result in a higher complication rate. ${ }^{23}$

In our hospital, the perineal wound is generally closed primarily. In the literature, some alternatives for surgical reconstruction of the perineum are described that might give less wound complications. Some studies reported that an omentoplasty could decrease the risk of wound dehiscence and improve overall healing. ${ }^{25}$ However, omentoplasty 
could increase the risk of incisional hernia and pneumonia and could delay gastric emptying. Another strategy to improve wound healing include reconstruction with myocutaneous flaps. Nisar and Scott ${ }^{26}$ published in 2009 an evidence-based review of reconstruction of pelvic defects after APR, using rectus abdominis, gracilis, and gluteus maximus flaps. Despite potential disadvantages of flaps, such as increased operation time, donor site morbidity, and the risk of flap necrosis, they concluded that immediate perineal reconstruction with myocutaneous flaps can result in significant improvements in perineal wound healing after APR with neoadjuvant radiotherapy for anorectal cancer. Because of the disadvantages of omentoplasty and flap reconstruction, these strategies generally were not applied in our hospital, but given the high rate of perineal wound complications in this study, it seems worthwhile to use an alternative technique for closure of the perineal wound in selected patients.

Kerr et al. (2008) $)^{6}$ investigated in 189 patients whether the interval between neoadjuvant chemoradiotherapy and surgery for rectal cancer influences postoperative morbidity or prognosis. They showed that shorter intervals independently predicted anastomotic leakage and perineal wound complications, and suggested delaying surgery beyond eight weeks. This finding could not be confirmed in present series or in other studies (Table 5).,11,13-16,20-22 A longer interval in this study also was not significantly associated with a higher $\mathrm{pCR}$ rate. Overall $\mathrm{pCR}$ rate was $22 \%$. This is in line with percentages, ranging from $10-24 \%$, reported by other investigators. ${ }^{3,4,6,10-13}$ The effect of a longer interval on complete tumor response remains unclear. In the literature twelve studies can be found

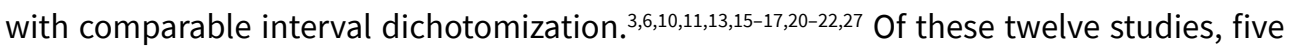
studies were able to show a higher $\mathrm{pCR}$ rate in patients with a longer interval, $3,10,11,15,16$ and seven studies were not (Table 5). 6,13,17,20-22,27 These conflicting results might be explained by differences in sample size, used definition of $\mathrm{pCR}$, used neoadjuvant chemoradiation regimen, and differences in dichotomization of intervals (as shown in Table 5). In studies were oxaliplatin was added to the 5-FU/capecitabine, a higher pathologic complete response was achieved than 5 -FU alone. ${ }^{16,27}$ In studies were irinotecan was added, no additional complete tumor response was seen..$^{13,20,22}$

Surprisingly, the youngest patient group developed more often postoperative complications. This is not in line with results in other studies. It was expected that elderly patients were prone to complications because of their comorbidities and the physiological factors that play a role in aging. ${ }^{5}$ It may be possible that surgeons took more risk during surgery in younger patients. On the contrary, patients in the two oldest age groups developed more often major complications (grade 3-5), although not significant. Perhaps, we selected only relatively fit elderly patients with locally advanced rectal cancer to undergo the intensive treatment regimen. Less fit patients are nowadays pretreated with a short course radiotherapy and long interval until surgery. ${ }^{2}$ Puig-La Calle 
and colleague ${ }^{28}$ also compared perioperative morbidity in primary rectal cancer patients, older and younger than 75 years of age. Perioperative complications were observed in $34 \%$ of elderly and $36 \%$ of younger patients. Elderly patients, if carefully selected, may not be less suitable than younger patients to undergo chemoradiation followed by rectal surgery.

In the present series, patients who received blood transfusions were four times more likely to have postoperative complications or postoperative grade 3-5 complications. This could possibly be explained by the immunomodulatory effect of blood transfusions. ${ }^{29} \mathrm{It}$ is suggested that allogeneic blood alters the immune response in a way that may render the recipient vulnerable to infection, the recurrence of malignancy, or the reactivation of latent viruses. ${ }^{29}$ Gruttadauria et al. $(2010)^{30}$ also found that patients who received intraoperative blood transfusions during liver resection for colorectal metastasis were four times more likely to experience (grade 3-5) postoperative complications. A limitation of the present study is that we did not distinguish between an intra- or postoperative blood transfusion. It may be possible that some patients received a blood transfusion because of a complication, although only $4 \%$ of patients had a bleeding as postoperative complication. Therefore, it seems more logical that the majority of the patients received a transfusion because of the intraoperative blood loss together with a low preoperative $\mathrm{Hb}$. Given the potential relationship between blood transfusions and the risk of postoperative complications, it seems important to perform blood transfusion only if necessary.

Another independent predictor of grade 3-5 complications was a lower ypT stage. Patients with more downstaging of their tumor were more likely to have major complications. The chemoradiotherapy in these patients achieved the goal of downstaging, but possibly also caused more damage to the surrounding healthy tissues resulting in an increased risk of major complications. This was also seen by others. Duldulao et al. $(2011)^{31}$ showed that patients with complete tumor response were more likely to have grade 3-5 postoperative complications. In contrast to these results, Stelzmueller et al. (2009) ${ }^{4}$ showed a significant association between grade 3-5 complications and a higher ypT stage.

Due to the retrospective nature of this study, it is subject to potential bias and certain limitations. Not all information on every patient was available. Optimal time interval between neoadjuvant therapy and surgery was decided based on results in literature, but factors such as patient morbidity and logistical scheduling also influenced the actual interval. Findings of a single-centre observational study such as this should be interpreted with caution. Another limitation of this study is that we did not investigate the influence of intrinsic tumor characteristics on $\mathrm{PCR}$ rate. Moreover, we were not able to identify prognostic factors of $\mathrm{pCR}$, presumably because of the limited number of patients. 


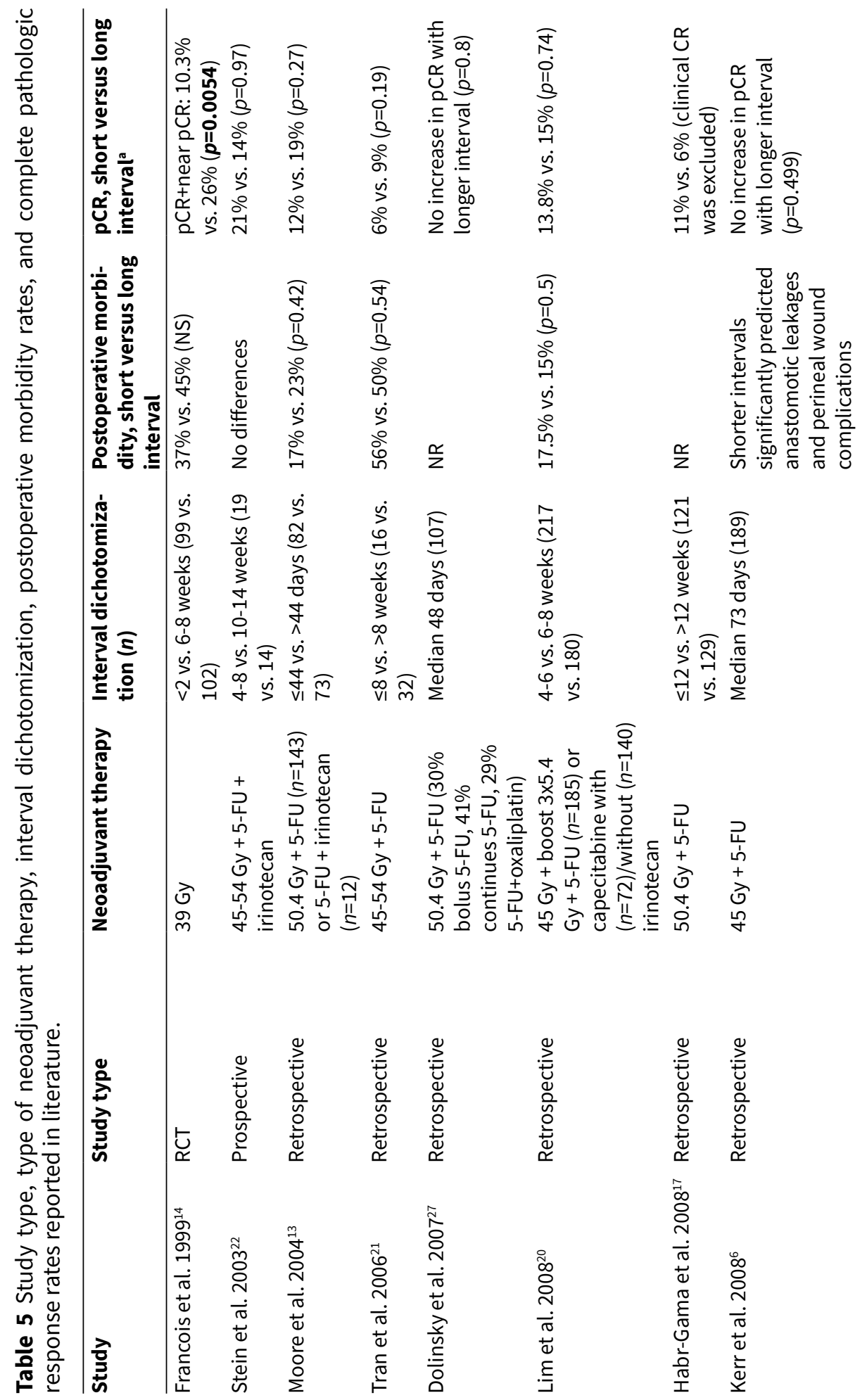




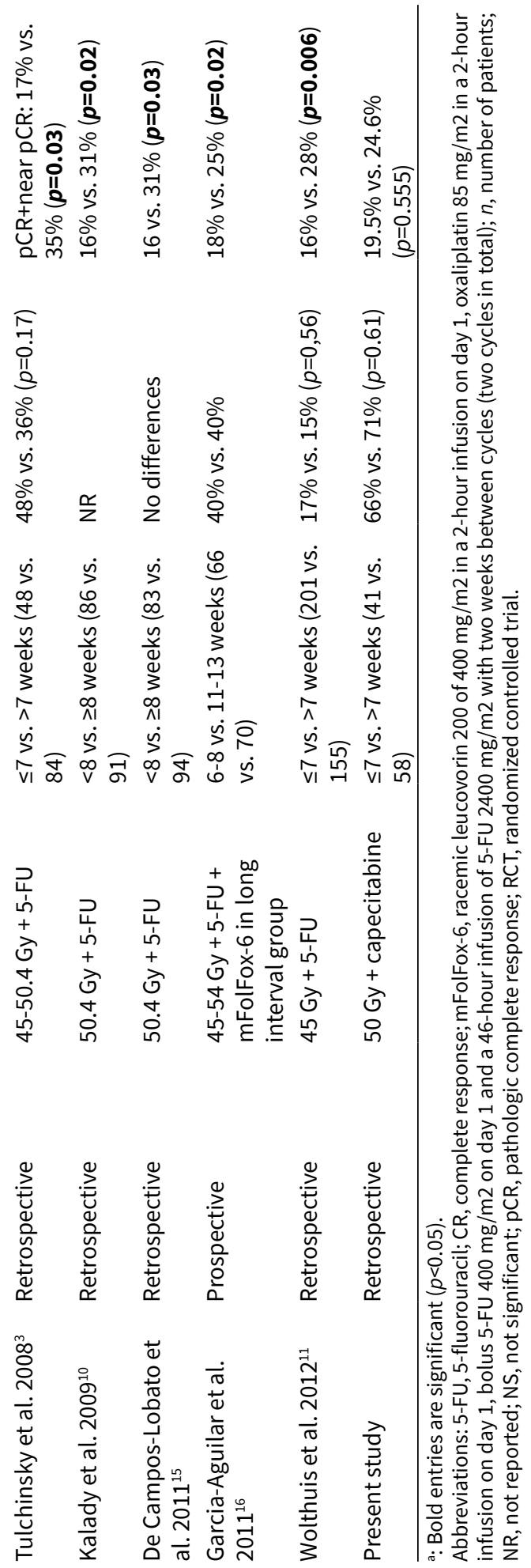




\section{Conclusion}

Neoadjuvant chemoradiation followed by rectal surgery is associated with significant postoperative morbidity, but minimal postoperative mortality. Age, blood transfusions, and ypT stage are associated with complications. Elderly patients, if carefully selected, may not be less suitable than younger patients to undergo the intensive treatment regimen. A complete response rate of $22 \%$ can be achieved, but no clinical prognostic factors could be found. 


\section{References}

1. Grossmann I, Klaase JM, Avenarius JK, de Hingh IH, Mastboom WJ, Wiggers T. The strengths and limitations of routine staging before treatment with abdominal CT in colorectal cancer. BMC Cancer 2011; 11: 433.

2. Landelijke werkgroep Gastro Intestinale Tumoren. Landelijke evidence-based richtlijn rectumcarcinoom. 2008; Available at: http://www.oncoline.nl/rectumcarcinoom.

3. Tulchinsky H, Shmueli E, Figer A, Klausner JM, Rabau M. An interval $>7$ weeks between neoadjuvant therapy and surgery improves pathologic complete response and disease-free survival in patients with locally advanced rectal cancer. Ann Surg Oncol 2008; 15(10): 2661-7.

4. Stelzmueller I, Zitt M, Aigner F, et al. Postoperative morbidity following chemoradiation for locally advanced low rectal cancer. J Gastrointest Surg 2009; 13(4): 657-67.

5. Dutch Surgical Colorectal Audit Jaarrapportage. 2011; Available at: http://www.dsca.nl/ images/documenten/2012/jaarrapportage\%202011.pdf.

6. Kerr SF, Norton S, Glynne-Jones R. Delaying surgery after neoadjuvant chemoradiotherapy for rectal cancer may reduce postoperative morbidity without compromising prognosis. Br J Surg 2008; 95(12): 1534-40.

7. Swellengrebel HA, Marijnen CA, Verwaal VJ, et al. Toxicity and complications of preoperative chemoradiotherapy for locally advanced rectal cancer. Br J Surg 2011; 98(3): 418-26.

8. Janjan NA, Khoo VS, Abbruzzese J, et al. Tumor downstaging and sphincter preservation with preoperative chemoradiation in locally advanced rectal cancer: the M. D. Anderson Cancer Center experience. Int J Radiat Oncol Biol Phys 1999; 44(5): 1027-38.

9. Rodel C, Martus P, Papadoupolos T, et al. Prognostic significance of tumor regression after preoperative chemoradiotherapy for rectal cancer. J Clin Oncol 2005; 23(34): 8688-96.

10. Kalady MF, de Campos-Lobato LF, Stocchi L, Geisler DP, Dietz D, Lavery IC, Fazio VW. Predictive factors of pathologic complete response after neoadjuvant chemoradiation for rectal cancer. Ann Surg 2009; 250(4): 582-9.

11. Wolthuis AM, Penninckx F, Haustermans K, De Hertogh G, Fieuws S, Van Cutsem E, D'Hoore A. Impact of interval between neoadjuvant chemoradiotherapy and TME for locally advanced rectal cancer on pathologic response and oncologic outcome. Ann Surg Oncol 2012; 19(9): 2833-41.

12. Das P, Skibber JM, Rodriguez-Bigas MA, et al. Predictors of tumor response and downstaging in patients who receive preoperative chemoradiation for rectal cancer. Cancer 2007; 109(9): 1750-5.

13. Moore HG, Gittleman AE, Minsky BD, et al. Rate of pathologic complete response with increased interval between preoperative combined modality therapy and rectal cancer resection. Dis Colon Rectum 2004; 47(3): 279-86.

14. Francois $\mathrm{Y}$, Nemoz CJ, Baulieux J, et al. Influence of the interval between preoperative radiation therapy and surgery on downstaging and on the rate of sphincter-sparing surgery for rectal cancer: the Lyon R90-01 randomized trial. J Clin Oncol 1999; 17(8): 2396. 
15. de Campos-Lobato LF, Geisler DP, da Luz Moreira A, Stocchi L, Dietz D, Kalady MF. Neoadjuvant therapy for rectal cancer: the impact of longer interval between chemoradiation and surgery. J Gastrointest Surg 2011; 15(3): 444-50.

16. Garcia-Aguilar J, Smith DD, Avila K, Bergsland EK, Chu P, Krieg RM, Timing of Rectal Cancer Response to Chemoradiation Consortium. Optimal timing of surgery after chemoradiation for advanced rectal cancer: preliminary results of a multicenter, nonrandomized phase II prospective trial. Ann Surg 2011; 254(1): 97-102.

17. Habr-Gama A, Perez RO, Proscurshim I, Nunes Dos Santos RM, Kiss D, Gama-Rodrigues J, Cecconello I. Interval between surgery and neoadjuvant chemoradiation therapy for distal rectal cancer: does delayed surgery have an impact on outcome? Int J Radiat Oncol Biol Phys 2008; 71(4): 1181-8.

18. Clavien PA, Barkun J, de Oliveira ML, et al. The Clavien-Dindo classification of surgical complications: five-year experience. Ann Surg 2009; 250(2): 187-96.

19. Dindo D, Demartines N, Clavien PA. Classification of surgical complications: a new proposal with evaluation in a cohort of 6336 patients and results of a survey. Ann Surg 2004; 240(2): 205-13.

20. Lim SB, Choi HS, Jeong SY, et al. Optimal surgery time after preoperative chemoradiotherapy for locally advanced rectal cancers. Ann Surg 2008; 248(2): 243-51.

21. Tran CL, Udani S, Holt A, Arnell T, Kumar R, Stamos MJ. Evaluation of safety of increased time interval between chemoradiation and resection for rectal cancer. Am J Surg 2006; 192(6): 8737.

22. Stein DE, Mahmoud NN, Anne PR, et al. Longer time interval between completion of neoadjuvant chemoradiation and surgical resection does not improve downstaging of rectal carcinoma. Dis Colon Rectum 2003; 46(4): 448-53.

23. Bullard KM, Trudel JL, Baxter NN, Rothenberger DA. Primary perineal wound closure after preoperative radiotherapy and abdominoperineal resection has a high incidence of wound failure. Dis Colon Rectum 2005; 48(3): 438-43.

24. Wiatrek RL, Thomas JS, Papaconstantinou HT. Perineal wound complications after abdominoperineal resection. Clin Colon Rectal Surg 2008; 21(1): 76-85.

25. Hay JM, Fingerhut A, Paquet JC, Flamant Y. Management of the pelvic space with or without omentoplasty after abdominoperineal resection for carcinoma of the rectum: a prospective multicenter study. The French Association for Surgical Research. Eur J Surg 1997; 163(3): 199206.

26. Nisar PJ, Scott HJ. Myocutaneous flap reconstruction of the pelvis after abdominoperineal excision. Colorectal Dis 2009; 11(8): 806-16.

27. Dolinsky CM, Mahmoud NN, Mick R, et al. Effect of time interval between surgery and preoperative chemoradiotherapy with 5-fluorouracil or 5-fluorouracil and oxaliplatin on outcomes in rectal cancer. J Surg Oncol 2007; 96(3): 207-12. 
28. Puig-La Calle J,Jr, Quayle J, Thaler HT, et al. Favorable short-term and long-term outcome after elective radical rectal cancer resection in patients 75 years of age or older. Dis Colon Rectum 2000; 43(12): 1704-9.

29. Klein HG. Immunomodulatory aspects of transfusion: a once and future risk? Anesthesiology 1999; 91(3): 861-5.

30. Gruttadauria S, Saint Georges Chaumet M, Pagano D, et al. Impact of blood transfusion on early outcome of liver resection for colorectal hepatic metastases. J Surg Oncol 2011; 103(2): 140-7.

31. Duldulao MP, Lee W, Le M, et al. Surgical complications and pathologic complete response after neoadjuvant chemoradiation in locally advanced rectal cancer. Am Surg 2011; 77(10): 1281-5. 



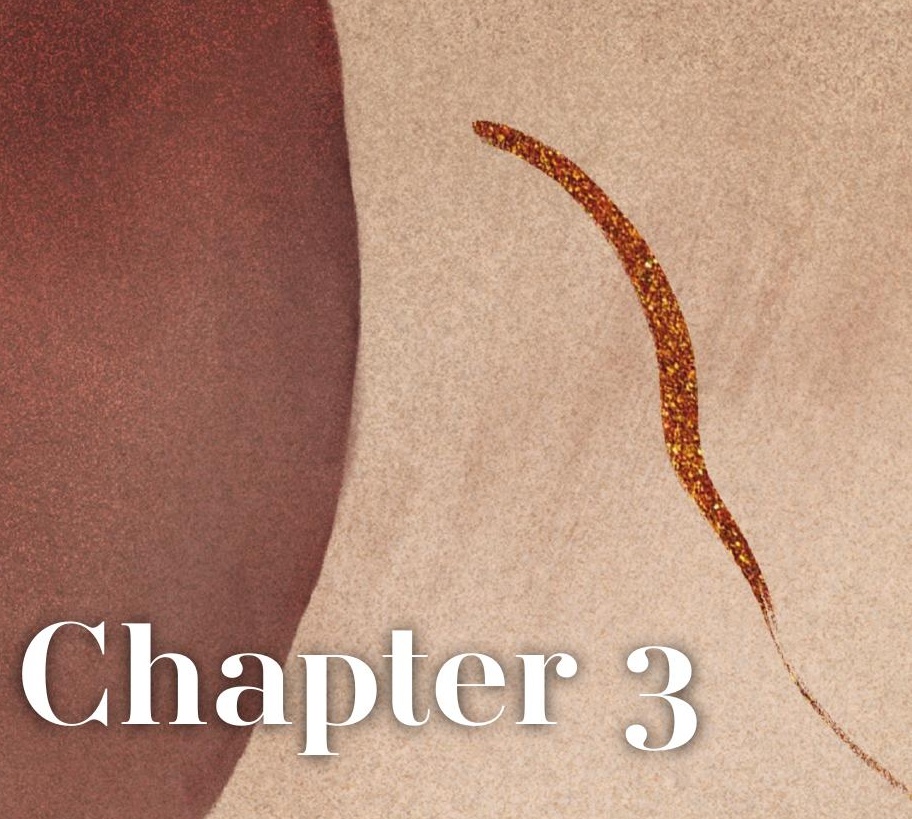

\section{Patient's skeletal muscle radiation} attenuation and sarcopenic obesity dreassociated with postoperative morbidity after neoadjuvant chemoradiation and resection for rectal cancer

Annefleur E.M. Berkel, Joost M. Klaase, Feike de Graaff, Marjolein G.J. Brusse-Keizer, Bart C. Bongers, Nico L.U. van Meeteren 


\section{Abstract}

Background: To investigate the relation between skeletal muscle measurements (muscle mass, radiation attenuation, and sarcopenic obesity), and postoperative morbidity, and survival after treatment of locally advanced rectal cancer.

Methods: This explorative retrospective study identified 99 consecutive patients who underwent neoadjuvant chemoradiation and surgery between January 2007 and May 2012. Skeletal muscle mass was measured as total psoas area and total abdominal muscle area (TAMA) at three anatomical levels using the patient's preoperative computed tomography scan. Radiation attenuation was measured using corresponding mean Hounsfield Units for TAMA. Sarcopenic obesity was defined as body mass index above $25 \mathrm{~kg} \cdot \mathrm{m}^{-2}$ combined with skeletal muscle mass index below the sex-specific median. Postoperative complications were graded by using the Clavien-Dindo classification.

Results: Twenty-five patients (25.3\%) developed a grade 3-5 complication. Lower radiation attenuation was independently associated with overall $(p=0.003)$ and grade $3-5$ complications ( $p=0.002$ ). Sarcopenic obesity was associated with overall complications (all $p<0.05$ ). Skeletal muscle measurements and survival were not significantly related.

Conclusion: Radiation attenuation was associated with overall and grade 3-5 postoperative morbidity after neoadjuvant chemoradiation and non-laparoscopic resection for rectal cancer. Sarcopenic obesity was associated with overall complications. 


\section{Introduction}

Treatment of locally advanced rectal cancer consists of administration of neoadjuvant chemoradiation followed by surgery; ${ }^{1}$ this treatment method however results in $40 \%$ postoperative complication rate. ${ }^{2,3}$ There is increasing evidence that sarcopenia (indicated by low skeletal muscle mass, assessed by measurement of muscle cross-sectional area most often using the computed tomography [CT] scan) is associated with poor clinical outcomes. Sarcopenia is associated with increased infectious complications, prolonged hospital stay, and decreased survival rates following colorectal resection and hepatic resection for colorectal liver metastases. ${ }^{4,5}$ There is also strong evidence to suggest that obesity is a significant risk factor for wound and stoma site complications after colorectal surgery. ${ }^{6}$ Combination of sarcopenia and obesity may result in even worse outcomes, since it combines the health risks of obesity and depleted lean muscle mass. ${ }^{7}$ Sarcopenic obesity is associated with reduced functional status and survival in patients with solid tumors of respiratory and gastrointestinal tracts. ${ }^{7}$ In patients undergoing liver resection for colorectal liver metastasis, sarcopenic obesity was associated with an increased risk of severe postoperative complications compared with patients who did not have sarcopenia. ${ }^{8}$

Timely identification of preoperative risk factors for adverse postoperative outcome will help patients and clinicians with treatment decisions, and with the selection of patients who might have a therapeutic window that leaves room for preoperative improvement of their functional health status known to protect for postoperative side effects., ${ }^{9,10}$ The aim of this explorative study was to investigate the relation between skeletal muscle mass, radiation attenuation (expressed in mean Hounsfield Units [HU] for total abdominal muscle area [TAMA]) and sarcopenic obesity, and postoperative morbidity and survival after the administration of neoadjuvant chemoradiation followed by non-laparoscopic resection for rectal cancer.

\section{Methods}

\section{Patients}

A retrospective study was performed including all consecutive patients with locally advanced rectal cancer who were treated with chemoradiation and surgery at our institute between January 2007 and May 2012. This is the same population dataset as we used in a previous article. ${ }^{11}$ In the current study however, we added data of patient's skeletal muscle mass, radiation attenuation, sarcopenic obesity, and survival.

Patients were included in the study if they had locally advanced rectal cancer (defined as a clinical T4 tumor or T3 tumor with a threatened circumferential margin (CRM $\leq 1 \mathrm{~mm}$ ) and/ 
or cN2 disease) ${ }^{1}$ that was histologically proven with a colonoscopy and biopsy. Patients were excluded if the preoperative abdominal CT scan was missing or if the abdominal CT scan was of poor quality.

\section{Neoadjuvant chemoradiation and surgery}

Patients underwent a five-week protocol of concomitant chemotherapy $(825 \mathrm{mg} \cdot \mathrm{m}$

2 capecitabine twice daily) and 3D-conformal radiotherapy (50 Gy in 25 fractions). Operations were carried out by experienced colorectal surgeons, according to the total mesorectal excision principle and consisted of abdominoperineal resection, low anterior resection (LAR) or rectal resection with definitive colostomy (Hartmann procedure). The considered optimal interval between chemoradiation and surgery in 2007-2010 was six to eight weeks, from 2010-2011 eight to ten weeks, and since 2012, it was ten to twelve weeks.

\section{Outcome variables}

The baseline characteristics included are age (divided in four groups based on quartiles), sex, body height, body mass, American Society of Anesthesiologists (ASA) score (I-IV), Charlson comorbidity score, smoking, clinical TNM stage, neoadjuvant chemoradiation regimen, symptoms of obstruction requiring fecal diversion before rectal surgery, interval between the end of chemoradiation and resection, complications of chemoradiation, type of surgery, diverting stoma (only for patients undergoing LAR), operation time, estimated blood loss, blood transfusion intra- or postoperative, pre-treatment tumor distance from the anal verge, length of hospital stay, postoperative surgical and general complications, readmission rate, mortality, pathologic tumor response, pathologic TNM stage, completeness of surgical excision ( $R$ stage), carcinoembryonic antigen (CEA) level before neoadjuvant chemoradiation, preoperative hemoglobin $(\mathrm{Hb})$, skeletal muscle mass, radiation attenuation, and sarcopenic obesity.

Prespecified definitions were used to score postoperative surgical complications (until six months after surgery). ${ }^{11}$ Complications were graded by using the Clavien-Dindo classification (grade 1-5). ${ }^{12,13}$ An overall complication is a grade 1-5 complication and a grade 3-5 complication is a severe complication. General complications were divided in cardiovascular, pulmonary, renal, or neurological complications.

Survival was defined as overall survival, measured in months from the day of surgery, with a follow-up of three years. The electronic patient record system was searched for date of death and/or the general practitioner was contacted.

\section{Muscle mass measurements and definitions}

Preoperative CT scans were imported in TeraRecon (TeraRecon Aquarius; TeraRecon, USA). 
Skeletal muscle mass was exploratively measured at three levels, because in the literature these different levels are used:8,14,15 cross-sections at the third lumbar vertebra (L3) where both transverse processes were visible, and at both the superior and inferior border of the fourth lumbar vertebra (L4). At each level, the cross-sectional areas $\left(\mathrm{cm}^{2}\right)$ of total psoas area (TPA) and total abdominal muscle area (TAMA), and skeletal muscle radiation attenuation (mean HU of TAMA) were measured. TPA was measured by manually tracing and adding left and right psoas area. TAMA was manually detected, and a threshold of -29 to $+150 \mathrm{HU}$ was applied to this region to discriminate between skeletal muscle and other tissues. ${ }^{16}$ Muscle area and average HU were then calculated automatically. Muscle areas were normalized for the patient' body height to calculate the muscle index for TPA and TAMA in $\mathrm{cm}^{2} \cdot \mathrm{m}^{-2}$. A lower $\mathrm{HU}$ value was considered to be related to a poorer overall skeletal muscle radiation attenuation, since lower $\mathrm{HU}$ values correspond with fatty infiltration of muscle (myosteatosis). ${ }^{17}$ The investigator who performed the measurements (FdG) was blinded for postoperative outcome. In the literature, measurement of cross-sectional muscle area has shown to have a low level of inter-observer variability, ${ }^{18,19}$ however, their muscle measurement methodology differed from the one we followed in our study.

Sarcopenia was defined as skeletal muscle mass index (TPA or TAMA at each level) below the median, calculated for males and females separately (sex specific cut-off value). ${ }^{20}$ Sarcopenic obesity was defined as body mass index (BMI) above $25 \mathrm{~kg} \cdot \mathrm{m}^{-2}$ combined with skeletal muscle mass index, TPA or TAMA, below the sex-specific median..$^{20,21}$

\section{Statistical analysis}

Data were analyzed with the Statistical Package for the Social Sciences for Windows (version 22.0; IBM, SPSS Inc., Chicago, IL, USA). Data are presented as mean and standard deviation for continuous normally distributed variables as checked with histograms. For continuous non-parametric variables, median and range were presented. Univariate associations between skeletal muscle mass measurements (TPA, TAMA, and radiation attenuation), sarcopenia, and sarcopenic obesity with overall and grade 3-5 postoperative complications were evaluated for significance, using Fisher exact test or $\mathrm{Chi}^{2}$ test for categorical variables and independent t-test (if normally distributed) or Mann Whitney U test for continuous variables. Possible confounders (see baseline characteristics above) were first tested for association with overall and grade 3-5 postoperative complications. In case they were associated with overall or grade 3-5 postoperative complications $(p<0.150)$, they were tested for association with the skeletal muscle mass measurements (TPA, TAMA, and radiation attenuation), sarcopenia and sarcopenic obesity $(p<0.150)$. The associations between skeletal muscle mass measurements (TPA, TAMA, and radiation attenuation), sarcopenia, and sarcopenic obesity with overall and grade 3-5 postoperative complications were corrected for the remaining potential confounders in a stepwise forward multivariate logistic regression analysis. In case of multicollinearity between 
variables, the variable that produced the best model fit (based on the -2 log likelihood) was included in the model. Kaplan-Meier estimates and Cox regression analyses were used to assess the association between sarcopenia and sarcopenic obesity with overall survival. All baseline characteristics that were associated with either sarcopenia and sarcopenic obesity $(p<0.150)$ were tested for an association with overall survival. The variables that were also associated with survival were taken into account in the stepwise forward multivariate Cox regression analyses. Statistical significance was determined at $p<0.05$.

\section{Results}

We identified 105 patients with locally advanced rectal cancer who underwent neoadjuvant chemoradiation between January 2007 and May 2012. After exclusion of six patients (patients with disease progression, extensive comorbidity, or unresectable tumors), resection of the tumor was performed in 99 patients (96.1\%, of which 53, 53.5\%, males), with a median age of 66 (range 40-81) years. Sixty-four patients (64.6\%) were overweight $(\mathrm{BMI} \geq 25)$. Eleven patients $(11.1 \%)$ had a BMI $30-35$, and six patients $(6.1 \%)$ had a BMI $\geq 35$. Median (range) muscle index for TAMA at L3 was $52.2(37.0-70.9) \mathrm{cm}^{2} \cdot \mathrm{m}^{-2}$ in men and 39.9 $(30.2-75.4) \mathrm{cm}^{2} \cdot \mathrm{m}^{-2}$ in women. We were able to retrieve all data of all consecutive patients, except body height and/or body mass $(n=3,3.0 \%)$, operation time $(n=2,2.0 \%)$, the CEA level before neoadjuvant chemoradiation ( $n=33,33.3 \%)$, and in four patients $(4.0 \%)$ the skeletal muscle mass/quality could not be measured on one or more levels because of vertebral screws ( $n=1,1.0 \%)$, poor quality of the CT scan $(n=1,1.0 \%)$, or the area of interest was not completely visible on CT scan ( $n=2,2.0 \%)$. In two $(2.0 \%)$ additional patients the skeletal muscle mass could not be measured because their body height was unknown.

Of the 99 patients that underwent resection, 68 patients $(68.7 \%)$ had an overall postoperative complication, of which 43 patients (43.4\%) had a grade $1-2$ complication and 25 patients $(25.3 \%)$ a grade 3-5 (severe) complication. An anastomotic leakage was seen in 2 of 18 patients undergoing LAR, a sepsis was seen in 6 of the 99 patients $(6.1 \%)$, and an abdominal wound complication in 17 of the 99 patients (17.2\%). The mortality rate was $2.0 \%$ (two patients). A complete overview of all postoperative complications can be found in Table 3 of our previous study ${ }^{11}$ or in supplementary file 1 (see www.karger. com/doi/10.1159/000490069). The median follow-up after surgery was 32.9 months (interquartile range 19.4-51.1 months). In the univariate relation between demographic and clinical variables and overall postoperative complications, a younger age $(p=0.017)$, an intra- or postoperative blood transfusion $(p=0.021)$, and a longer operation time $(p=0.093)$ were associated with postoperative complications $(p<0.150)$. In the univariate relation between demographic and clinical variables and grade 3-5 postoperative complications, 
male sex $(p=0.032)$, a higher Charlson comorbidity score $(p=0.134)$, a lower CEA score before chemoradiation ( $p=0.082)$, pathological T (ypT) stage $(p=0.092)$, and an intra- or postoperative blood transfusion ( $p=0.004$ ) were associated with grade 3-5 postoperative complications $(p<0.150)$. A complete overview of these results can be found in Table 4 of our previous article ${ }^{11}$ or in online supplementary file 2 . An intra- or postoperative blood transfusion was not taken into account as a confounder in the multivariate analysis since the necessity of intra- or postoperative blood transfusion could be an intermediary factor between skeletal muscle mass and postoperative complications (e.g., patients could have had a blood transfusion because of a postoperative complication, for example due to bleeding).

\section{Postoperative morbidity and skeletal muscle mass/quality}

Skeletal muscle mass (TPA and TAMA) was not significantly associated with overall or grade 3-5 complications. In the univariate analysis, a lower skeletal muscle radiation attenuation at all three measurements levels was significantly associated with overall and grade 3-5 complications (Table 1). TPA at L4 superior and L4 inferior were associated $p<0.150$ with grade 3-5 complications and were therefore also assessed in the multivariate analysis for grade 3-5 complications. Due to multicollinearity between radiation attenuation measurements (L3, L4 inferior, and L4 superior level) and between TPA measurements (L4 inferior and L4 superior), the variable of radiation attenuation and TPA with the best model fit was included in the multivariate logistic regression analysis (radiation attenuation at L3 for both overall and grade 3-5 complications and TPA at L4 superior for grade 3-5 complications).

Of the potential confounders that were assessed for an association with overall postoperative complications, age and operation time were also associated with radiation attenuation and taken into account in the multivariate analysis for postoperative complications. In the final multivariate model (Table 2), lower skeletal muscle radiation attenuation was associated with an increased risk of overall postoperative complications (OR 0.91; 95\% Cl=0.85-0.97; $p=0.003$ ), as was young age (OR 0.92; 95\% Cl=0.87-0.98; $p=0.007)$.

For postoperative grade 3-5 (severe) complications, TPA at L4 superior and radiation attenuation, as well as the potential confounders sex, Charlson comorbidity score, CEA before chemoradiation, and ypT stage were included in the multivariate analysis. A lower radiation attenuation (OR $0.89 ; 95 \% \mathrm{Cl}=0.83-0.96 ; p=0.002)$ and a higher total psoas mass at $\mathrm{L} 4$ superior (OR 1.46; $95 \% \mathrm{Cl}=1.10-1.94 ; p=0.009$ ) were associated with an increased risk of grade $3-5$ complications (Table 2 ). 


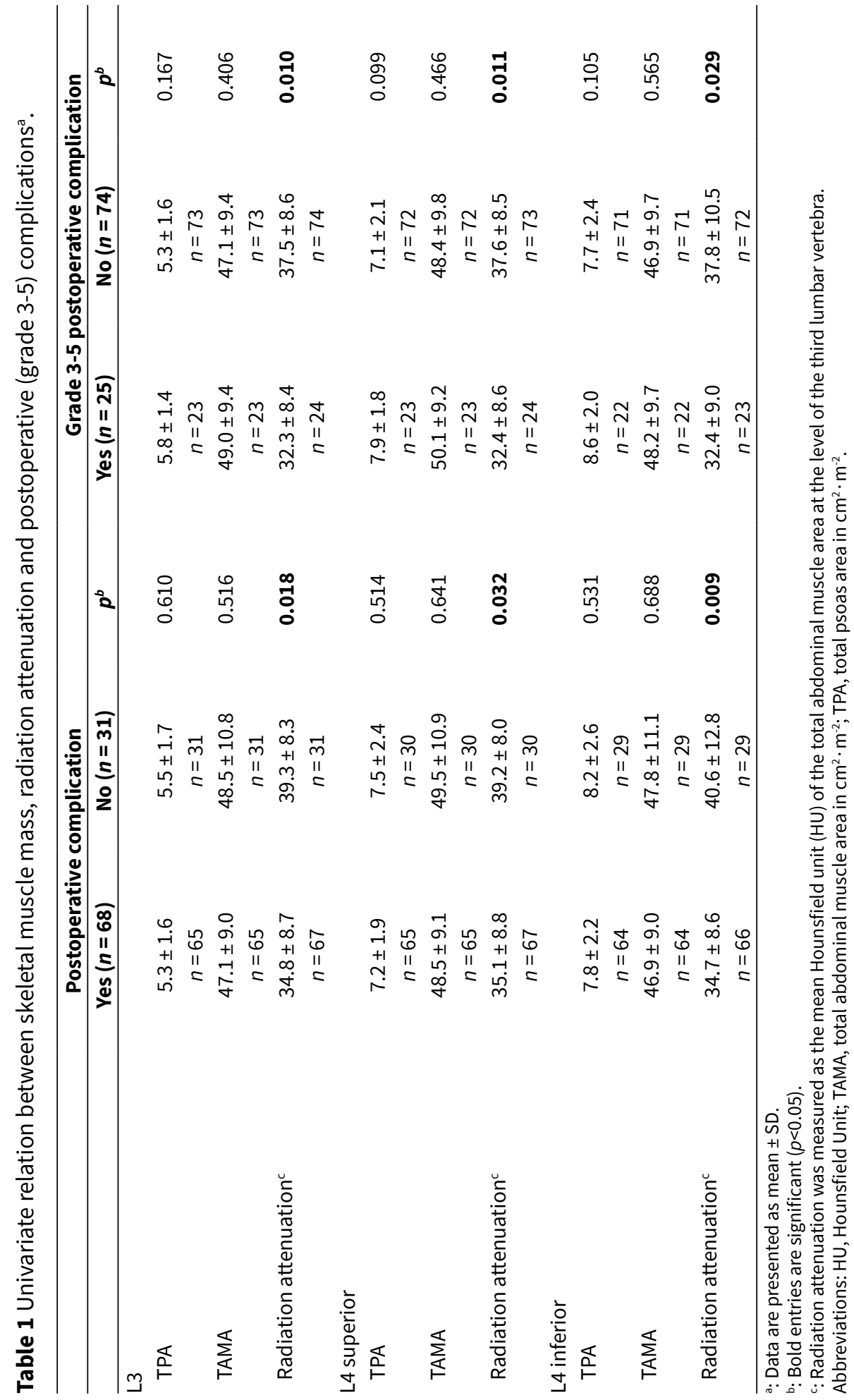


Table 2 Univariate and multivariate relation between skeletal muscle mass, radiation attenuation and postoperative (grade 3-5) complications ${ }^{\mathrm{a}}$.

\begin{tabular}{|c|c|c|c|c|}
\hline & $\begin{array}{l}\text { OR univariate } \\
(95 \% \mathrm{CI})\end{array}$ & $p$ univariate & $\begin{array}{c}\text { OR multivariate } \\
(95 \% \mathrm{CI})\end{array}$ & p multivariate \\
\hline \multicolumn{5}{|l|}{$\begin{array}{l}\text { Postoperative } \\
\text { complications }\end{array}$} \\
\hline Radiation attenuation ${ }^{b}$ & $0.94(0.89-0.99)$ & 0.021 & $0.91(0.85-0.97)$ & 0.003 \\
\hline Age (years) & $0.95(0.90-1.00)$ & 0.063 & $0.92(0.87-0.98)$ & 0.007 \\
\hline Operation time (min) & $1.01(1.00-1.02)$ & 0.098 & - & - \\
\hline \multicolumn{5}{|l|}{ Grade 3-5 complications } \\
\hline TPA L4 superior & $1.20(0.96-1.51)$ & 0.103 & $1.46(1.10-1.94)$ & 0.009 \\
\hline Radiation attenuation ${ }^{b}$ & $0.93(0.88-0.99)$ & 0.013 & $0.89(0.83-0.96)$ & 0.002 \\
\hline Sex & & & - & - \\
\hline Female & Ref & & & \\
\hline Male & $2.87(1.07-7.67)$ & 0.036 & & \\
\hline $\begin{array}{l}\text { Charlson comorbidity } \\
\text { score }\end{array}$ & & & - & - \\
\hline 0 & Ref & 0.056 & & \\
\hline$>1$ & $2.49(0.98-6.36)$ & & & \\
\hline CEA before CRT & $0.98(0.93-1.02)$ & 0.318 & - & - \\
\hline урт stage & & & - & - \\
\hline урт0-1 & Ref & & & \\
\hline урT2-4 & $0.43(0.17-1.09)$ & 0.076 & & \\
\hline
\end{tabular}

a: Blood transfusion was not taken into account as a confounder in the multivariate analysis since the necessity of intra- or postoperative blood transfusion could be an intermediary factor between skeletal muscle mass and postoperative complications.

${ }^{b}$ : Radiation attenuation was measured as the mean Hounsfield unit (HU) of the total abdominal muscle area at the level of the third lumbar vertebra.

No value means that the confounder was removed from the equation, because it did not significantly predicted complications in the multivariate model.

Abbreviations: CEA, carcinoembryonic antigen; CRT, chemoradiation; TPA, total psoas area in $\mathrm{cm}^{2} \cdot \mathrm{m}^{-2}$; ypT, pathological T stage.

\section{Postoperative morbidity and sarcopenia}

In the univariate analysis, sarcopenia was not significantly associated with overall and grade 3-5 postoperative complications. Sarcopenia measured as TPA at L4 superior was the only variable with $p<0.150$ that seemed to be associated with grade $3-5$ complications. However, in the multivariate model, this association was not statistically significant (OR $0.51 ; 95 \% \mathrm{Cl}=0.20-1.29 ; p=0.152$ ).

\section{Postoperative morbidity and sarcopenic obesity}

In the univariate analysis, sarcopenic obesity was not significantly associated with overall complications. However, sarcopenic obesity when measured at TPA L4 superior, TAMA L3, and TAMA L4 superior were associated with overall complications with $p<0.150$ and were therefore included in three separate multivariate analyses, together with confounders 
(age and operation time). In the final multivariate models, sarcopenic obesity measured at TPA L4 superior, TAMA L3, and TAMA L4 superior, and young age were significantly associated with postoperative complications (all $p<0.05$; Table 3 ). Patients with sarcopenic obesity were three times more likely (ORs between 3.2 and 3.8) to develop a postoperative complication.

No significant relation was found between sarcopenic obesity and grade 3-5 (severe) complications.

Table 3 Multivariate relation between sarcopenic obesity and postoperative complications.

\begin{tabular}{lccc}
\hline & OR & $\mathbf{9 5 \%} \mathbf{C l}$ & $\boldsymbol{p}$ \\
\hline L3 & & & \\
TAMA & 3.77 & $1.12-12.66$ & 0.032 \\
Age (years) & 0.94 & $0.89-0.99$ & 0.030 \\
L4 superior & & & \\
TPA & 3.21 & $1.05-9.77$ & 0.040 \\
Age (years) & 0.94 & $0.90-1.00$ & 0.033 \\
L4 superior & & & \\
TAMA & 3.42 & $1.02-11.42$ & 0.046 \\
Age (years) & 0.94 & $0.90-1.00$ & 0.034 \\
\hline
\end{tabular}

Abbreviations: TAMA, total abdominal muscle area in $\mathrm{cm}^{2} \cdot \mathrm{m}^{-2} ;$ TPA, total psoas area in $\mathrm{cm}^{2} \cdot \mathrm{m}^{-2}$.

\section{Overall survival and sarcopenia}

In the univariate analysis, sarcopenia at TAMA L4 inferior was the only variable that was associated with overall survival with a $p<0.150$ ( $\mathrm{HR} 2.04 ; 95 \% \mathrm{Cl}=0.83-4.99 ; p=0.120$ ). Of all baseline characteristics (see Method section) that were assessed for an association with overall survival, ASA, BMI, and tumor regression were also associated with sarcopenia at TAMA L4 inferior $(p<0.150)$ and taken into account in the multivariate analysis for overall survival. In the multivariate model, controlled for ASA, BMI, and tumor regression, none of the potential confounders remained in the multivariate model after the stepwise regression. Therefore, the association between sarcopenia at TAMA L4 inferior and survival remained the same.

\section{Overall survival and sarcopenic obesity}

No significant relation was found between sarcopenic obesity and survival with $p>0.337$.

\section{Discussion}

This retrospective study found that a lower skeletal muscle radiation attenuation was associated with overall and grade 3-5 (severe) postoperative morbidity after 
chemoradiation and resection for rectal cancer. Sarcopenic obesity was associated with overall complications. A few previous studies investigated the influence of skeletal muscle radiation attenuation on postoperative complications following colorectal cancer resection. Sabel et al. ${ }^{22}$ showed that a lower radiation attenuation was the best predictor of any complication after colectomy for colon cancer. Boer et al. ${ }^{20}$ showed that a lower radiation attenuation was associated with overall complications after open colon resection for cancer. Body composition might be of key interest to use in predicting outcome.

More studies investigated the role of sarcopenia on complications after colorectal surgery. Sarcopenia was associated with more infectious complications, ${ }^{5}$ higher mortality, ${ }^{18}$ and decreased survival ${ }^{23}$ after colorectal cancer resection. Reisinger et al. ${ }^{24}$ evaluated whether low muscle mass was associated with increased inflammation after resection of colorectal malignancies by measuring inflammatory markers in plasma. They found that a low muscle mass was associated with an increased postoperative inflammatory response, which may be a part of the explanation for the high incidence of postoperative complications in sarcopenic patients. ${ }^{24}$ In the present study, no significant relation between sarcopenia and postoperative complications was found. Sarcopenia is more than only a low skeletal muscle mass measured at a single time point. Sarcopenia is characterized by loss of skeletal muscle mass, skeletal muscle strength, and/or physical performance. ${ }^{25}$ Defining sarcopenia only in terms of muscle mass might be too narrow and may therefore be of limited clinical value. ${ }^{25} \mathrm{An}$ example is the finding in the present study that patients with a higher muscle mass (TPA at L4 superior), more often had a grade 3-5 complication. Muscle mass might be not depleted in cross-sectional area because of more fat infiltration of the muscle. Fat infiltration is the most widely accepted cause of reduced attenuation of muscle. ${ }^{26}$ Measurement of radiation attenuation is potentially a better approach. Future research should investigate if muscle strength measurements of a muscle biopsy (rectus abdominis muscle) correlates with CT measurements.

Sarcopenic obesity could be of higher clinical use than sarcopenia alone. In the present study, sarcopenic obesity was associated with overall complications. Previously, sarcopenic obesity was found to be a predictor for grade 3-5 postoperative complications following open colon cancer surgery, ${ }^{20}$ and an independent predictor of survival in patients with solid tumors in the respiratory or gastrointestinal tract. ${ }^{7}$ After hepatic resection for colorectal liver metastasis, patients with sarcopenic obesity had a more pronounced risk of severe complications compared with patients without sarcopenia (sarcopenic obesity $40 \%$ versus non-sarcopenia $8 \%, p=0.02) .{ }^{8}$ Visser et $\mathrm{al}^{27}$ showed that sarcopenic obesity independently increased the risk of postoperative infections in patients undergoing cardiac surgery.

The current study underscores the importance of assessing skeletal muscle mass and 
quality measurements to select high-risk patients that might benefit from preoperative optimization (prehabilitation) to prevent a dismal course. Studies show that highrisk patients who were placed on a physical exercise training program before major elective surgery, improve their physical fitness, ${ }^{28-30}$ even after $^{31}$ or during ${ }^{10}$ neoadjuvant chemoradiation in patients with rectal cancer. It remains to be seen, however, whether improving physical fitness increases skeletal muscle mass, and vice versa. Currently, in our hospital, we perform a randomized controlled trial (www.trialregister.nl, trial registration number NTR4032) to measure the effect of a three-week prehabilitation program on postoperative complications in high-risk patients who are to undergo colorectal surgery, ${ }^{32}$ all in accordance with a part of the recent advises of Hulzebos and Van Meeteren. ${ }^{9}$ In this trial, the effect of the prehabilitation program on muscle strength will also be investigated.

Since undergoing a CT scan is usual care in the work up of all patients with rectal cancer, no additional test is needed to evaluate skeletal muscle mass and radiation attenuation. Skeletal muscle mass and radiation attenuation measurements could potentially be used as a case-mix correction factor in the Dutch Surgical Colorectal Audit (DSCA), a quality registration of colorectal surgery in the Netherlands, to make a more accurate comparison of outcome among various hospitals. Future research should reveal optimal skeletal muscle index and quality cutoff points in this specific population. Moreover, it would be of interest to investigate the association between skeletal muscle measurements and oncological outcome and prognosis.

Our study has some limitations. Definitions of sarcopenia and sarcopenic obesity remain under debate. We used definitions based on previous studies, ${ }^{20,21}$ which are different than those used in several other studies. A different definition could have led to different results. Moreover, the univariate associations between muscle mass index and outcome, as well as between muscle radiation attenuation and outcome were not calculated for males and females separately, while body composition is not the same between sexes. We used BMI for the definition of sarcopenic obesity based on previous research, ${ }^{7}$ however, adipose tissue measurements on the CT scan might be superior to the calculation of BMI. The explorative nature of the skeletal muscle mass analyses at three different levels might be both a limitation as well as a strength of our study. For future research, using level L3 might be recommended..$^{33}$ But, it remains to be seen whether or not using L3 CTslices is the best approach. Due to the retrospective and explorative nature of this study, and the limited number of patients, it is subject to potential bias and certain limitations with respect to their internal validity and generalization. Our findings should therefore be interpreted with caution. More research is needed to confirm our results. Since surgery took place between 2007 and 2012, no laparoscopic procedures were performed. Nowadays, to follow a laparoscopic approach is more common and known to result in less complications, ${ }^{34}$ but the results here may of course only account for non-laparoscopic 
surgical approaches.

\section{Conclusion}

In this explorative manuscript, we found that skeletal muscle radiation attenuation was associated with overall and grade 3-5 (severe) postoperative morbidity after chemoradiation and non-laparoscopic resection for rectal cancer. Sarcopenic obesity was associated with overall complications. Skeletal muscle mass and sarcopenia were not associated with postoperative complications. Body composition might be used to identify patients with high risk of worse outcome after surgery, paving the way to select patients that might benefit from preoperative optimization.

\section{Acknowledgements}

This study was previously presented at the 10th Sarcopenia, Cachexia and Wasting Disorders (SCWD) conference in Rome, Italy, 8-10 December 2017 and the abstract was published in the Journal of Cachexia Sarcopenia and Muscle 2017 (reference: Abstracts of the 10th International Conference on Cachexia, Sarcopenia and Muscle Wasting, Rome, Italy, 8-10 December 2017 (Part 1). Journal of Cachexia, Sarcopenia and Muscle, 8:1019. doi:10.1002/jcsm.12255). This is an open access article under the terms of the Creative Commons Attribution License, which permits use, distribution, and reproduction in any medium, provided the original work is properly cited. 


\section{References}

1. Landelijke werkgroep Gastro Intestinale Tumoren. Landelijke evidence-based richtlijn colorectaal carcinoom. 2014; Available at: http://www.oncoline.nl/colorectaalcarcinoom. Accessed March, 2015.

2. Tulchinsky H, Shmueli E, Figer A, Klausner JM, Rabau M. An interval $>7$ weeks between neoadjuvant therapy and surgery improves pathologic complete response and disease-free survival in patients with locally advanced rectal cancer. Ann Surg Oncol 2008; 15(10): 2661-7.

3. Stelzmueller I, Zitt M, Aigner F, et al. Postoperative morbidity following chemoradiation for locally advanced low rectal cancer. J Gastrointest Surg 2009; 13(4): 657-67.

4. van Vledder MG, Levolger S, Ayez N, Verhoef C, Tran TC, ljzermans JN. Body composition and outcome in patients undergoing resection of colorectal liver metastases. Br J Surg 2012; 99(4): 550-7.

5. Lieffers JR, Bathe OF, Fassbender K, Winget M, Baracos VE. Sarcopenia is associated with postoperative infection and delayed recovery from colorectal cancer resection surgery. $\mathrm{Br} J$ Cancer 2012; 107(6): 931-6.

6. Gendall KA, Raniga S, Kennedy R, Frizelle FA. The impact of obesity on outcome after major colorectal surgery. Dis Colon Rectum 2007; 50(12): 2223-37.

7. Prado CM, Lieffers JR, McCargar LJ, Reiman T, Sawyer MB, Martin L, Baracos VE. Prevalence and clinical implications of sarcopenic obesity in patients with solid tumours of the respiratory and gastrointestinal tracts: a population-based study. Lancet Oncol 2008; 9(7): 629-35.

8. Peng PD, van Vledder MG, Tsai S, et al. Sarcopenia negatively impacts short-term outcomes in patients undergoing hepatic resection for colorectal liver metastasis. HPB (Oxford) 2011; 13(7): 439-46.

9. Hulzebos EH, van Meeteren NL. Making the elderly fit for surgery. Br J Surg 2016; 103(4): 463.

10. Heldens AF, Bongers BC, de Vos-Geelen J, van Meeteren NL, Lenssen AF. Feasibility and preliminary effectiveness of a physical exercise training program during neoadjuvant chemoradiotherapy in individual patients with rectal cancer prior to major elective surgery. Eur J Surg Oncol 2016; 42(9): 1322-30. doi: 10.1016/j.ejso.2016.03.021.

11. Berkel AE, Woutersen DP, van der Palen J, Klaase JM. Prognostic factors for postoperative morbidity and tumour response after neoadjuvant chemoradiation followed by resection for rectal cancer. J Gastrointest Surg 2014; 18(9): 1648-57.

12. Clavien PA, Barkun J, de Oliveira ML, et al. The Clavien-Dindo classification of surgical complications: five-year experience. Ann Surg 2009; 250(2): 187-96.

13. Dindo D, Demartines N, Clavien PA. Classification of surgical complications: a new proposal with evaluation in a cohort of 6336 patients and results of a survey. Ann Surg 2004; 240(2): 205-13.

14. Lee JS, He K, Harbaugh CM, et al. Frailty, core muscle size, and mortality in patients undergoing open abdominal aortic aneurysm repair. J Vasc Surg 2011; 53(4): 912-7. 
15. Hasselager R, Gogenur I. Core muscle size assessed by perioperative abdominal CT scan is related to mortality, postoperative complications, and hospitalization after major abdominal surgery: a systematic review. Langenbecks Arch Surg 2014; 399(3): 287-95.

16. Mitsiopoulos N, Baumgartner RN, Heymsfield SB, Lyons W, Gallagher D, Ross R. Cadaver validation of skeletal muscle measurement by magnetic resonance imaging and computerized tomography. J Appl Physiol (1985) 1998; 85(1): 115-22.

17. Taaffe DR, Henwood TR, Nalls MA, Walker DG, Lang TF, Harris TB. Alterations in muscle attenuation following detraining and retraining in resistance-trained older adults. Gerontology 2009; 55(2): 217-23.

18. Reisinger KW, van Vugt JL, Tegels JJ, et al. Functional compromise reflected by sarcopenia, frailty, and nutritional depletion predicts adverse postoperative outcome after colorectal cancer surgery. Ann Surg 2015; 261(2): 345-52.

19. Shen W, Punyanitya M, Wang Z, et al. Total body skeletal muscle and adipose tissue volumes: estimation from a single abdominal cross-sectional image. J Appl Physiol (1985) 2004; 97(6): 2333-8.

20. Boer BC, de Graaff F, Brusse-Keizer M, Bouman DE, Slump CH, Slee-Valentijn M, Klaase JM. Skeletal muscle mass and quality as risk factors for postoperative outcome after open colon resection for cancer. Int J Colorectal Dis 2016; Jun;31(6): 1117-24.

21. Jaap K, Hunsinger M, Dove J, et al. Morphometric predictors of morbidity after pancreatectomy. Am Surg 2016 Dec; 82(12): 1221-1226.

22. Sabel MS, Terjimanian M, Conlon AS, et al. Analytic morphometric assessment of patients undergoing colectomy for colon cancer. J Surg Oncol 2013; 108(3): 169-75.

23. Miyamoto $\mathrm{Y}$, Baba $\mathrm{Y}$, Sakamoto $\mathrm{Y}$, et al. Sarcopenia is a Negative Prognostic Factor After Curative Resection of Colorectal Cancer. Ann Surg Oncol 2015; 22(8): 2663-8.

24. Reisinger KW, Derikx JP, van Vugt JL, et al. Sarcopenia is associated with an increased inflammatory response to surgery in colorectal cancer. Clin Nutr 2016; 35(4): 924-7.

25. Cruz-Jentoft AJ, Baeyens JP, Bauer JM, et al. Sarcopenia: European consensus on definition and diagnosis: Report of the European Working Group on Sarcopenia in Older People. Age Ageing 2010; 39(4): 412-23.

26. Aubrey J, Esfandiari N, Baracos VE, Buteau FA, Frenette J, Putman CT, MazurakVC. Measurement of skeletal muscle radiation attenuation and basis of its biological variation. Acta Physiol (Oxf) 2014; 210(3): 489-97.

27. Visser M, van Venrooij LM, Vulperhorst L, de Vos R, Wisselink W, van Leeuwen PA, de Mol BA. Sarcopenic obesity is associated with adverse clinical outcome after cardiac surgery. Nutr Metab Cardiovasc Dis 2013; 23(6): 511-8.

28. O'Doherty AF, West M, Jack S, Grocott MP. Preoperative aerobic exercise training in elective intra-cavity surgery: a systematic review. Br J Anaesth 2013; 110(5): 679-89.

29. Barberan-Garcia A, Ubre M, Roca J, et al. Personalized Prehabilitation in High-risk Patients Undergoing Elective Major Abdominal Surgery: A Randomized Blinded Controlled Trial. Ann Surg 2018; 267(1): 50-6. 
30. Dunne DF, Jack S, Jones RP, et al. Randomized clinical trial of prehabilitation before planned liver resection. Br J Surg 2016; 103(5): 504-12.

31. West MA, Loughney L, Lythgoe D, et al. Effect of prehabilitation on objectively measured physical fitness after neoadjuvant treatment in preoperative rectal cancer patients: a blinded interventional pilot study. Br J Anaesth 2015; 114(2): 244-51.

32. Berkel AEM, Bongers BC, van Kamp MS, et al. The effects of prehabilitation versus usual care to reduce postoperative complications in high-risk patients with colorectal cancer or dysplasia scheduled for elective colorectal resection: study protocol of a randomized controlled trial. BMC Gastroenterol 2018; 18(1): 29,018-0754-6.

33. Mourtzakis M, Prado CM, Lieffers JR, Reiman T, McCargar LJ, Baracos VE. A practical and precise approach to quantification of body composition in cancer patients using computed tomography images acquired during routine care. Appl Physiol Nutr Metab 2008; 33(5): 9971006.

34. Kolfschoten NE, van Leersum NJ, Gooiker GA, et al. Successful and safe introduction of laparoscopic colorectal cancer surgery in Dutch hospitals. Ann Surg 2013; 257(5): 916-21. 



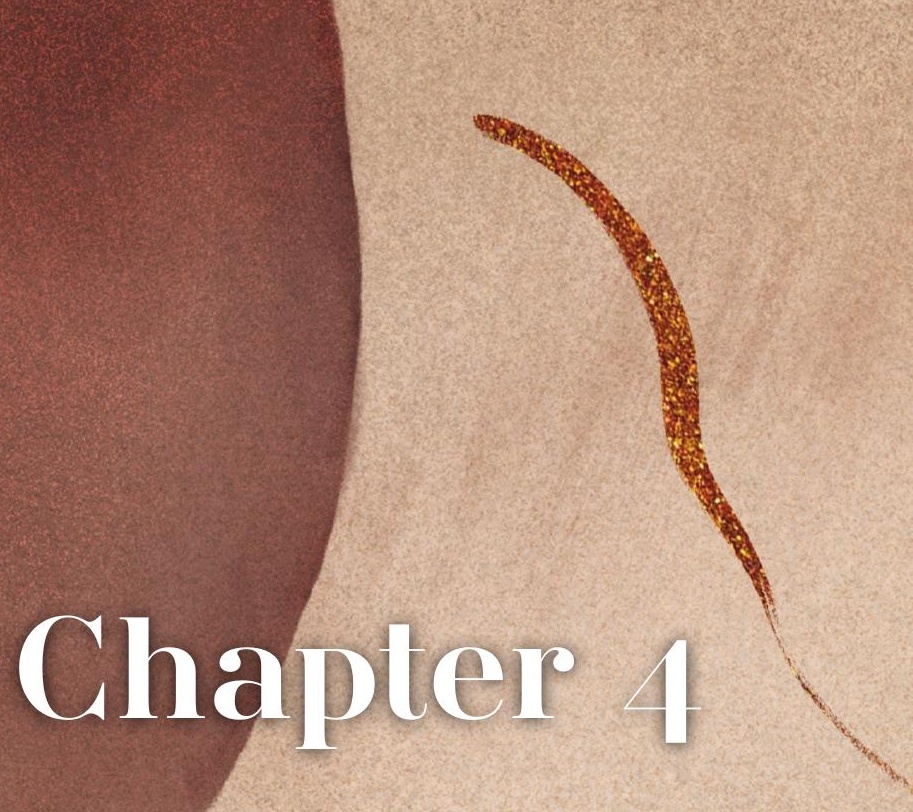

An evaluation of the validity of the preoperative oxygen uplake efficiency slope as an indicator of cardiorespitatory fitness in elderly patients scheduled for major colorectal surgery

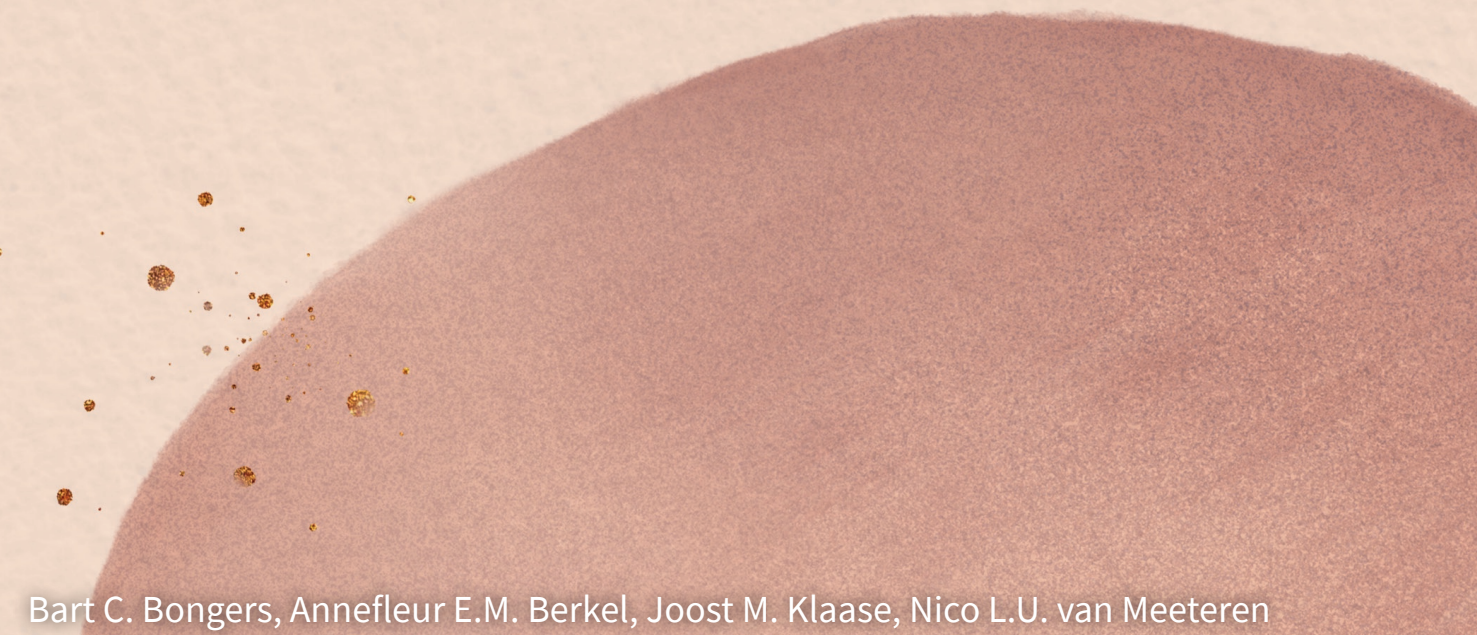

Bart C. Bongers, Annefleur E.M. Berkel, Joost M. Klaase, Nico L.U. van Meeteren 


\section{Abstract}

Background: This study aimed to investigate the validity of the oxygen uptake efficiency slope as an objective and submaximal indicator of cardiorespiratory fitness in elderly patients scheduled for major colorectal surgery.

Methods: Patients $\geq 60$ years of age, with a metabolic equivalent score at the veteransspecific activity questionnaire $\leq 7$ and scheduled for major colorectal surgery participated in a preoperative cardiopulmonary exercise test. The oxygen uptake efficiency slope was calculated up to different exercise intensities, using $100 \%, 90 \%$, and $80 \%$ of the exercise data.

Results: Data from 71 patients (47 males, mean (SD) age 75.2 (6.7) years) were analyzed. The efficiency slope obtained from all the data was statistically significantly different from the values when $90 \%(p=0.027)$ and $80 \%(p=0.023)$ of the data were used. The $90 \%$ and $80 \%$ values did not differ significantly from each other $(p=0.152)$. Correlations between the oxygen uptake efficiency slope and the peak oxygen uptake ranged from 0.816 to 0.825 (all $p<0.001$ ), and correlations between oxygen uptake efficiency slope and the ventilatory anaerobic threshold ranged from 0.793 to 0.805 (all $p<0.001$ ). Receiver operating characteristic curves showed that the oxygen uptake efficiency slope is a sensitive and specific predictor of a peak oxygen uptake $\leq 18.2 \mathrm{~mL} \cdot \mathrm{kg}^{-1} \cdot \mathrm{min}^{-1}$, with an area under the curve $(95 \% \mathrm{Cl})$ of $0.876(0.780-0.972, \mathrm{p}<0.001)$ and a ventilatory anaerobic threshold $\leq 11.1$ $\mathrm{mL} . \mathrm{kg}^{-1} \cdot \mathrm{min}^{-1}$, with an area under the curve $(95 \% \mathrm{Cl})$ of $0.828(0.726-0.929, \mathrm{p}<0.001)$.

Conclusion: These correlations suggest that the oxygen uptake efficiency slope provides a valid (sub)maximal measure of cardiorespiratory fitness in these patients, and the predictive ability described indicates that it might help discriminate patients at higher risk of postoperative morbidity. However, future research should investigate the prognostic value of the oxygen uptake efficiency slope for postoperative outcomes. 


\section{Introduction}

Cardiorespiratory fitness, as indicated by the highest measured oxygen uptake (peak oxygen uptake) and/or the oxygen uptake at the ventilatory anaerobic threshold, is widely recognized as an important measure in the evaluation of the risk of perioperative complications in major abdominal surgery. Similar to immunological and neuroendocrine reserve, cardiorespiratory fitness indicates the physiological reserve capacity of the patient to maintain homeostasis with adequate responses to allostatic load (allostasis). These are required to cope with the psychophysiological consequences of diagnosis, the preadmission period, and the stress of surgery. Those patients with higher cardiorespiratory fitness might be more resilient to the increased metabolic demands from these perioperative challenges. Preoperative cardiorespiratory fitness has been found to have a consistent relation with postoperative outcome indicators in major elective abdominal surgery (e.g., morbidity, mortality, length of stay). ${ }^{1-12}$

An incremental cardiopulmonary exercise test up to maximal exertion is considered the best test for assessing cardiorespiratory fitness. A recent systematic review ${ }^{13}$ demonstrated that peak oxygen uptake, and the oxygen uptake at the ventilatory anaerobic threshold, are the most commonly used preoperative cardiopulmonary exercise test measures recorded before major elective abdominal surgery. There are, however, several clinical limitations when determining cardiorespiratory fitness using peak oxygen uptake and/or the ventilatory anaerobic threshold; moreover, assessing only these two measures leads to substantial loss of physiological data. It would, therefore, be worthwhile evaluating how useful alternative objective (sub)maximal exercise parameters might be in assessing and predicting prognosis in patients undergoing major surgery. One such parameter, the oxygen uptake efficiency slope (OUES), has been reported to have prognostic value in predicting major cardiac events and mortality in patients with heart failure and coronary artery disease. ${ }^{14-17}$ The oxygen uptake efficiency slope concept is based on the curvilinear relationship between the minute ventilation and oxygen uptake throughout an incremental cardiopulmonary exercise test. Baba et al. ${ }^{18}$ introduced and validated a logarithmic transformation of the minute ventilation over the entire exercise period, resulting in a linear relationship between the minute ventilation and oxygen uptake during the entire, and especially the last part, of the cardiopulmonary exercise test. The regression coefficient of the regression line describing this linear relationship is the oxygen uptake efficiency slope. The theoretical linearity of the oxygen uptake efficiency slope implies that it does not require a maximal effort and can thus be derived rather accurately from submaximal exercise data as well. The oxygen uptake efficiency slope has been reported to correlate strongly with well-established measures of cardiorespiratory fitness such as peak oxygen uptake and the ventilatory anaerobic threshold in different groups of adults and children. ${ }^{18-30}$ The oxygen uptake efficiency slope has also been found to have excellent 
test-retest reliability in general surgical patients, with a reliability coefficient $(95 \% \mathrm{Cl})$ of 0.89 (0.81-0.97). ${ }^{31}$ However, the validity of the oxygen uptake efficiency slope as an effortindependent alternative measure of preoperative cardiorespiratory fitness, and its ability to predict postoperative outcomes, are unknown. To the best of our knowledge, only one study investigated the predictive value of the oxygen uptake efficiency slope for five-year survival after major surgery, and concluded that the oxygen uptake efficiency slope did not appear to be a strong predictor of mortality. ${ }^{32}$ Therefore, we aimed to investigate the validity of the oxygen uptake efficiency slope as an effort-independent indicator of cardiorespiratory fitness in elderly patients scheduled for major colorectal surgery, by correlating it with peak oxygen uptake and the ventilatory anaerobic threshold and assessing its stability throughout the last part of the cardiopulmonary exercise test.

\section{Methods}

All patients $\geq 60$ years of age requiring colorectal resection for colorectal cancer of dysplasia grade I-III at the Medisch Spectrum Twente (a non-academic teaching hospital in the Netherlands) were identified at the multi-disciplinary oncology meeting. They were seen at the outpatient clinic by the surgeon or surgical resident and asked to fill in the veteransspecific activity questionnaire. ${ }^{33}$ Patients with a metabolic equivalent score on the veterans-specific activity questionnaire of $\leq 7$ (low subjective metabolic equivalent score) ${ }^{4}$ were invited to participate in the study. Patients not able to perform a cardiopulmonary exercise test, or patients who were undergoing emergency colorectal surgery, were excluded. The study was approved by the Medical Research Ethics Committee of the Medisch Spectrum Twente, Enschede, the Netherlands, and written informed consent was obtained from each participant. After providing consent, the patient underwent a cardiopulmonary exercise test in the period between February 2013 and July 2016. These formed the study data and were retrospectively analyzed after this period.

All participants performed an incremental cardiopulmonary exercise test up to maximal exertion in upright position on an electronically-braked cycle ergometer (Ergoline, Ergoselect 100, Bitz, Germany). The seat height was adjusted to the participant's leg length. After assessment of baseline cardiopulmonary values during a two-minute rest period while seated at the cycle ergometer, patients performed a three-minute warm-up phase that consisted of unloaded cycling. After the warm-up, the work rate was increased by constant increments of 5,10 , or $15 \mathrm{~W} \cdot \mathrm{min}^{-1}$, depending on the patient's subjective physical fitness level, aiming to reach maximal effort within eight to twelve minutes. Throughout the cardiopulmonary exercise test, patients had to maintain a pedaling frequency of between 60 and 80 revolutions. $\mathrm{min}^{-1}$. The protocol continued until the patient's pedaling frequency fell definitely below 60 revolutions. $\mathrm{min}^{-1}$, despite strong verbal encouragement, or when 
the patient met the criteria for exercise termination before being limited by symptoms. ${ }^{34}$

During the cardiopulmonary exercise test, patients breathed through a facemask (Hans Rudolph, Kansas City, MO, USA) connected to an ergospirometry system (Oxycon Pro, Jaeger, Hoechberg, Germany) that was calibrated for respiratory gas analysis measurements (ambient air and a gas mixture of $16 \%$ oxygen and $5 \%$ carbon dioxide) and volume measurements (3 litre syringe). Expired gas was passed through a flow meter (Triple V volume sensor, Jaeger, Hoechberg, Germany), an oxygen analyzer, and a carbon dioxide analyzer. The flow meter and gas analyzers were connected to a computer, which calculated breath-by-breath minute ventilation, oxygen uptake, carbon dioxide production, and the respiratory exchange ratio averaged at 10-s intervals. Heart rate was measured by continuous 12-lead electrocardiography. A test was considered to be at or near the maximal level when participants: showed clinical signs of intense effort (e.g., unsteady biking, sweating, and clear unwillingness to continue exercising despite strong encouragement); were unable to maintain the required pedaling speed; and when at least one of the following criteria was met: a heart rate at peak exercise of $>95 \%$ of predicted (predicted peak heart rate [beats. $\left.\mathrm{min}^{-1}\right]=210-(0.8 \times$ age [years] $)$ ) or a respiratory exchange ratio at peak exercise of $>1.10$.

Cardiopulmonary parameters were averaged into ten-second intervals; after outliers ( $>3$ SD from the local mean) were removed. ${ }^{35}$ Data interpretation was performed independently from the clinicians involved, and without knowledge about the clinical status of the included patients, by a trained and experienced clinical exercise physiologist $(B C B)$. Absolute values at peak exercise were calculated as the average value over the last 30s before termination of the test. Peak heart rate was defined as the highest heart rate achieved during the cardiopulmonary exercise test. The ventilatory anaerobic threshold was defined as the point at which the ventilatory equivalent for oxygen and the partial end-tidal oxygen tension reached a minimum and thereafter began to rise in a consistent manner, coinciding with an unchanged ventilatory equivalent for carbon dioxide and partial end-tidal carbon dioxide tension. ${ }^{34}$ When this ventilatory equivalents method appeared to provide uncertain results for a patient's ventilatory anaerobic threshold, the point at which the linear slope of the relation between the carbon dioxide production and oxygen uptake changed was taken as the ventilatory anaerobic threshold, according to the V-slope method. ${ }^{36}$ The ventilatory anaerobic threshold was expressed as an absolute and relative value (normalized for body mass). The graphical presentation of the minute ventilation as a function of carbon dioxide production during the incremental cardiopulmonary exercise test was used to determine the point at which the minute ventilation increased out of proportion to carbon dioxide production, that is, the respiratory compensation point. The slope of the relationship between the minute ventilation and carbon dioxide production was calculated by linear least squares 
regression of the relation between the minute ventilation and carbon dioxide production up to the respiratory compensation point. The oxygen uptake efficiency slope was calculated using the following equation: oxygen uptake $=\mathrm{a} \times \log$ (minute ventilation) + $b$, in which the constant ' $a$ ' represents the rate of increase in oxygen uptake in response to an increase in minute ventilation, called the oxygen uptake efficiency slope (regression coefficient) and ' $b$ ' corresponds to the intercept. ${ }^{18}$ As depicted in Figure 1 , a steeper slope, reflected by a higher oxygen uptake efficiency slope, represents a more efficient oxygen uptake: a smaller ventilation quantity is required for a certain oxygen uptake. Data from the first minute of exercise were excluded because of the often very irregular breathing pattern at the onset of exercise. ${ }^{37}$ In order to evaluate the robustness of the oxygen uptake efficiency slope during the last part of the cardiopulmonary exercise test, it was calculated up to three different exercise intensities. Based on the findings in a previous study, ${ }^{38}$ it was calculated up to the point of the levelling-off of oxygen uptake in case of a clear oxygen uptake plateau (a decrease in oxygen uptake during the last 30 s with an increase in work rate). For the determination of the OUES100, all data gained during the cardiopulmonary exercise test were used (data as of the first minute after the start of the work rate increments, up to peak exercise or up to the oxygen uptake plateau), whereas for the determination of the OUES90 and the OUES 80 , only data up to $90 \%$ and $80 \%$ of the total exercise duration were used, respectively. Oxygen uptake efficiency slope values were also normalized for body mass (OUES. kg ${ }^{-1}$ ). Sex-, age-, and body surface area-related predictive equation $\mathrm{s}^{20}$ were used to express each patient's oxygen uptake efficiency slope value as a percentage of predicted.

All data were analyzed using the Statistical Package for the Social Sciences for Windows (version 22; IBM, SPSS Inc., Chicago, IL, USA). Tests for normality were performed on the data with the Shapiro-Wilk test. Variability in peak oxygen uptake, the ventilatory anaerobic threshold, and oxygen uptake efficiency slope were evaluated by calculating the coefficient of variation, defined as $100 \times(\mathrm{SD} /$ mean). To assess the construct validity of the oxygen uptake efficiency slope, repeated measures analysis of variance (ANOVA) was used to evaluate differences in oxygen uptake efficiency slope values calculated at the three different exercise intensities to indicate the effect of exercise duration on the oxygen uptake efficiency slope, thereby showing its linear characteristics. Additional post-hoc analyses, with Bonferroni adjustment for multiple testing, were performed on the outcomes of the repeated-measures ANOVA tests to locate the exact significant differences. Receiver operating characteristic curves were used to establish threshold values of oxygen uptake efficiency slope to predict a peak oxygen uptake $\leq 18.2 \mathrm{~mL} . \mathrm{kg}^{-1} \cdot \mathrm{min}$ ${ }^{1}$ or a ventilatory anaerobic threshold $\leq 11.1 \mathrm{~mL} \cdot \mathrm{kg}^{-1} \cdot \mathrm{min}^{-1}$. These peak oxygen uptake and ventilatory anaerobic threshold values are based on the cut-off points described by West et al. ${ }^{12}$ In addition, Pearson or Spearman correlation coefficients, as appropriate, were calculated to examine associations between the oxygen uptake efficiency slope and peak 
oxygen uptake and the ventilatory anaerobic threshold. To evaluate the group validity of the oxygen uptake efficiency slope, the total group was divided in a subgroup with a ventilatory anaerobic threshold $>11.1 \mathrm{~mL} \cdot \mathrm{kg}^{-1} \cdot \mathrm{min}^{-1}$ and a subgroup with a ventilatory anaerobic threshold $\leq 11.1 \mathrm{~mL} . \mathrm{kg}^{-1}$. $\mathrm{min}^{-1}$. Differences in oxygen uptake efficiency slope values and other exercise parameters between these subgroups were assessed using independent-samples t-tests or Mann-Whitney $\mathrm{U}$ tests, as appropriate. Significance was set a priori at the 0.05 level.
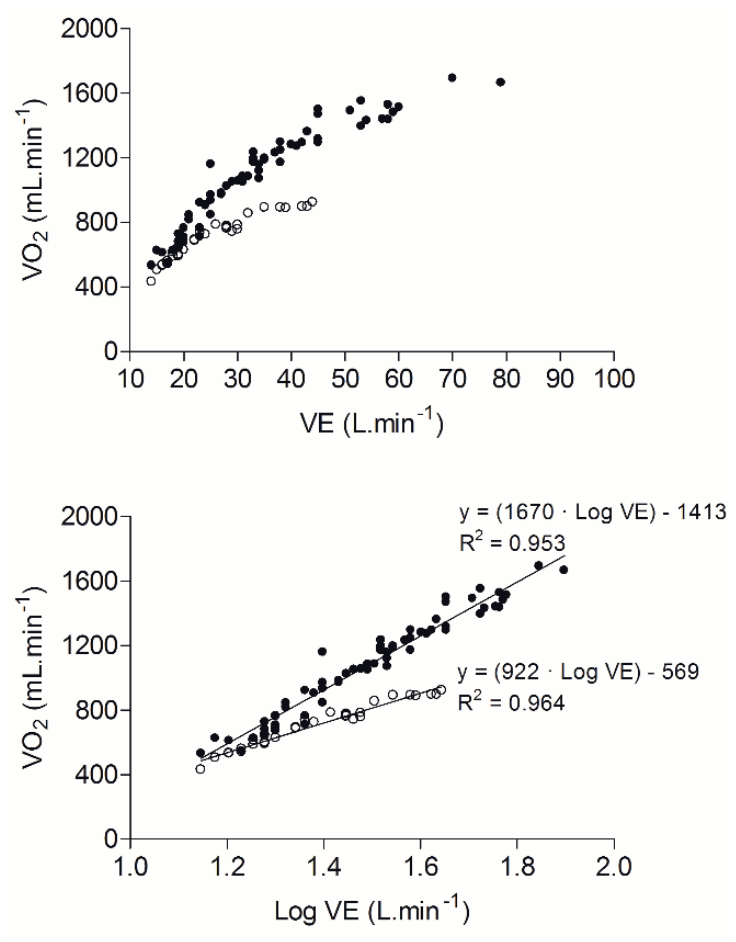

Figure 1 Relation between the oxygen uptake and the minute ventilation during the cardiopulmonary exercise test in a 71-year-old woman with a ventilatory anaerobic threshold of $12.3 \mathrm{~mL} \cdot \mathrm{kg}^{-1} \cdot \mathrm{min}^{-1}(\bullet)$ and in an 82 -year-old woman with a ventilatory anaerobic threshold of $9.1 \mathrm{~mL} \cdot \mathrm{kg}^{-1} \cdot \mathrm{min}^{-1}(\mathrm{O})$. The values of the oxygen uptake efficiency slope (calculated using all exercise data) are 1670 and 922, respectively, and the data are presented as linear (top graph) and semi-log plots of the $\mathrm{x}$-axis (bottom graph).

Abbreviations: Log VE, common logarithm of the minute ventilation; VE, minute ventilation; $\mathrm{VO}_{2}$, oxygen uptake. 


\section{Results}

Between February 2013 and July 2016, a sample of 72 eligible patients took part in this study and undertook a cardiopulmonary exercise test a mean (SD) of 26.8 (29.8) days before colorectal resection (Table 1). Cardiopulmonary exercise test data of a 73-year-old female patient were excluded because she terminated the test before she reached the ventilatory anaerobic threshold.

All 71 patients performed the cardiopulmonary exercise test without any complications or adverse events during or after the test. They all showed subjective signs of a maximal effort as described in the Methods section. Most patients $(n=54,76.1 \%)$ also showed objective signs of maximal effort, as indicated by a peak heart rate $>95 \%$ of predicted and/or a peak respiratory exchange ratio $>1.10$. A total of 19 patients $(26.8 \%)$ showed a clear oxygen uptake plateau during the last part of the cardiopulmonary exercise test. The oxygen uptake efficiency slope could be determined in all 71 patients, whereas the ventilatory anaerobic threshold was undeterminable in two (2.8\%) patients. Coefficients of variation were $27.2 \%$ for peak oxygen uptake, $19.0 \%$ for the ventilatory anaerobic threshold, and $28.2 \%$ for the OUES100. Normalized for body mass, coefficients of variation were $24.2 \%$ for peak oxygen uptake, $18.5 \%$ for the ventilatory anaerobic threshold, and $26.3 \%$ for the OUES100. All cardiopulmonary exercise test results are shown in Table 2. 
Table 1 Participant characteristics $(n=71)$.

\begin{tabular}{l}
\hline Variable \\
\hline Age (years) \\
Sex (males) \\
Body height $(\mathrm{cm})$ \\
Body mass $(\mathrm{kg})$ \\
Body mass index $\left(\mathrm{kg} \cdot \mathrm{m}^{-2}\right)$ \\
Body surface area $\left(\mathrm{m}^{2}\right)$
\end{tabular}

n $\%$

47

66.2

Mean (SD)

$75.1(6.7)$

$171.6(9.4)$

$84.3(14.1)$

$28.6(4.0)$

$2.0(0.2)$

Charlson comorbidity index

$0 \quad 18$

1

$2+$

Comorbidity

Cardiac disease $\quad 37$

Diabetes

Chronic obstructive pulmonary disease

Orthopedic disease

None of the above

Beta blockers

Yes

American Joint Committee on Cancer stage $e^{a, b}$

I

II

III

IV

American Society of Anesthesiologists score

I

II

III

IV

Surgical procedure

Right hemicolectomy

Transverse hemicolectomy

Left hemicolectomy

Sigmoid colectomy

Abdominoperineal resection

Low anterior resection

Hartmann

Extended right hemicolectomy

Extended left hemicolectomy

Other

No surgery

Method of surgery

Open
18
29

24

15

12

19

16

30

42.3

19

31.7

9

27

15.0

45.0

5

8.3

2.8

77.5

18.3

1.4

1

23

32.4

3

4.2

4.2

26.8

4.2

7.0

1.4

2.8

1.4

8.5

7.0

5

13

18.3 
Laparoscopic

Conversion to open

No surgery
46

7

5
64.8

9.9

7.0

lung metastasis $(n=1)$, so in this case $n=60$.

b: American Joint Committee on Cancer colon and rectum cancer staging ( $7^{\text {th }}$ edition).

The mean (SD) predicted oxygen uptake efficiency slope was 2001 (433); hence, the current patient group scored $79 \%$ (20\%) of predicted, with values ranging from 28 to $123 \%$ of predicted. A statistically significant difference was found between the OUES100 and the OUES90 ( $p=0.027)$, as well as between the OUES100 and the OUES80 ( $p=0.023)$, with lower OUES100 values. OUES90 and OUES 80 showed not statistically significant difference from each other $(p=0.152)$.

Compared to patients with a ventilatory anaerobic threshold $>11.1 \mathrm{~mL} \cdot \mathrm{kg}^{-1} \cdot \mathrm{min}^{-1}$, patients with a ventilatory anaerobic threshold $\leq 11.1 \mathrm{~mL} \cdot \mathrm{kg}^{-1} \cdot \mathrm{min}^{-1}$ had statistically significant lower oxygen uptake efficiency slope values (Table 2), which corresponds to the statistically significant lower values for peak oxygen uptake and the ventilatory anaerobic threshold in this subgroup.

Correlation coefficients between the oxygen uptake efficiency slope, determined at different relative exercise intensities, and peak oxygen uptake and the ventilatory anaerobic threshold are summarized in Table 4A.1 in Appendix 4.1. Figure 2 shows the relation between the oxygen uptake efficiency slope and peak oxygen uptake, between the oxygen uptake efficiency slope and the ventilatory anaerobic threshold, and between the ventilatory anaerobic threshold and peak oxygen uptake. When normalized for body mass, a correlation coefficient of $0.792(p<0.001)$ was observed between peak oxygen uptake and the ventilatory anaerobic threshold.

Receiver operating characteristic analysis depicted in Figure 3 shows the value of the oxygen uptake efficiency slope in predicting a peak oxygen uptake $\leq 18.2 \mathrm{~mL} . \mathrm{kg}^{-1} \cdot \mathrm{min}^{-1}$. The OUES100. $\mathrm{kg}^{-1}$ had an area under the curve $(95 \% \mathrm{Cl})$ of $0.876(0.780-0.972, p<0.001)$, the OUES90. $\mathrm{kg}^{-1}$ had an area under the curve $(95 \% \mathrm{Cl})$ of $0.880(0.779-0.981, p<0.001)$, and the OUES80. $\mathrm{kg}^{-1}$ had an area under the curve $(95 \% \mathrm{Cl})$ of $0.890(0.797-0.983, p<0.001)$. Figure 3 also shows the value of the oxygen uptake efficiency slope to predict a ventilatory anaerobic threshold $\leq 11.1 \mathrm{~mL} . \mathrm{kg}^{-1} \cdot \mathrm{min}^{-1}$. The OUES100. $\mathrm{kg}^{-1}$ had an area under the curve $(95 \% \mathrm{Cl})$ of $0.828(0.726-0.929, p<0.001)$, the OUES90. $\mathrm{kg}^{-1}$ had an area under the curve $(95 \%$ $\mathrm{Cl})$ of $0.840(0.743-0.938, p<0.001)$, and the OUES80. $\mathrm{kg}^{-1}$ had an area under the curve $(95 \%$ $\mathrm{Cl}$ ) of $0.859(0.769-0.948, p<0.001)$. Optimal cut-off values for oxygen uptake efficiency slope including sensitivity and specificity can be found in Table 3. 


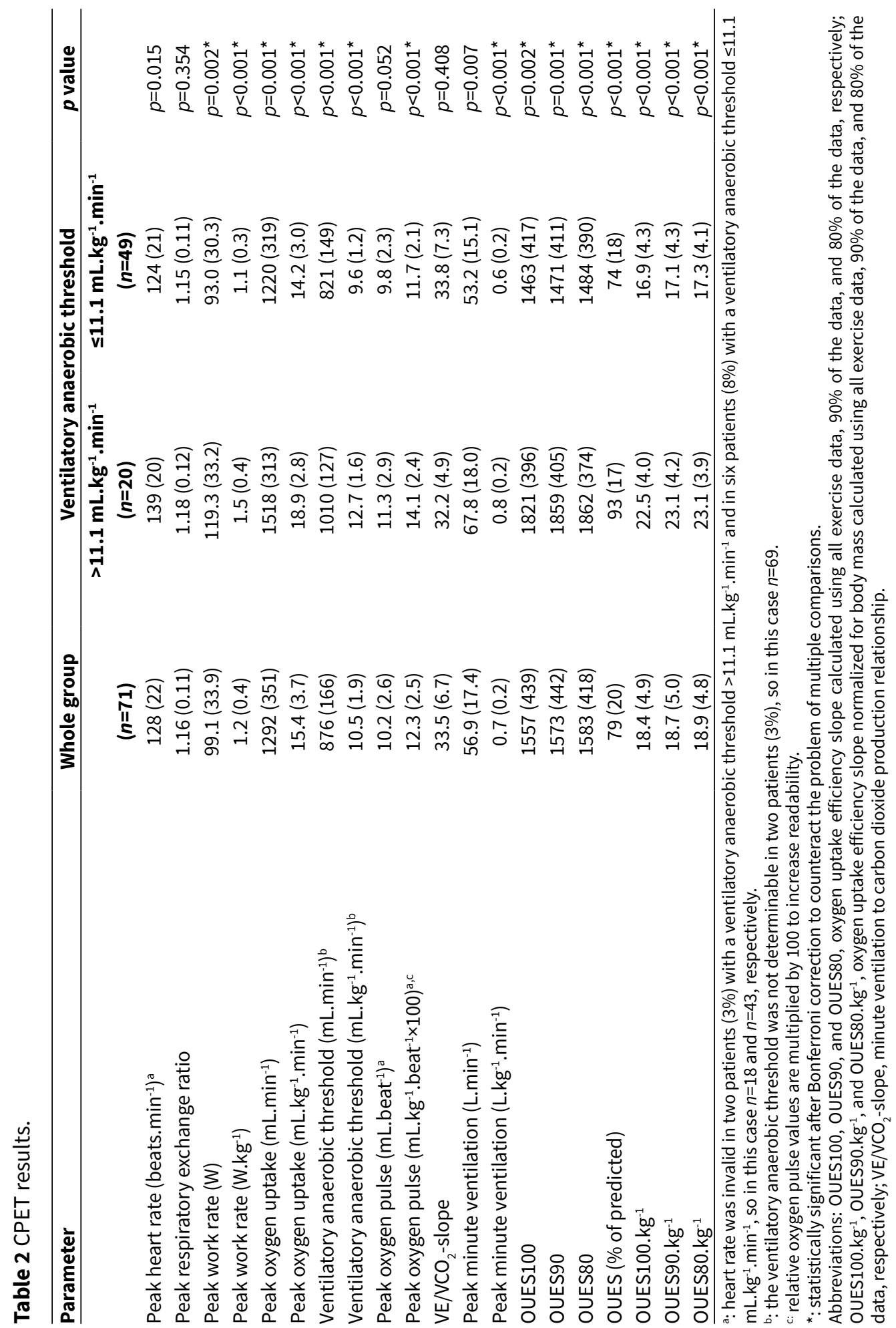




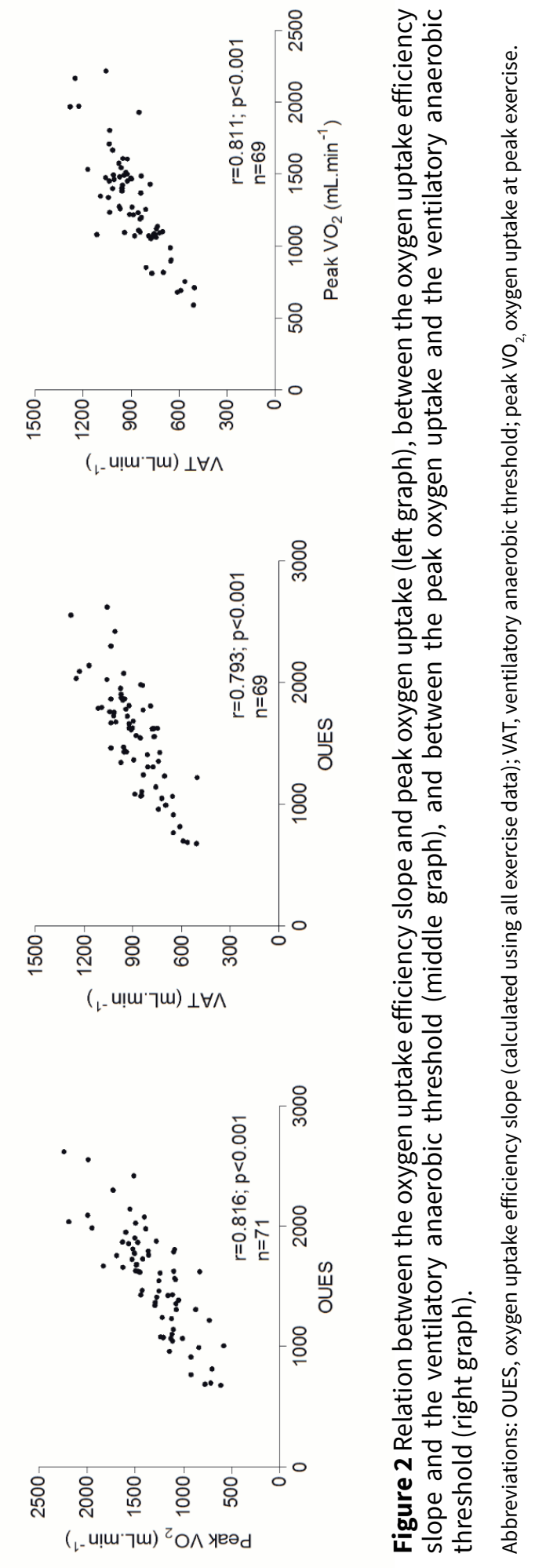



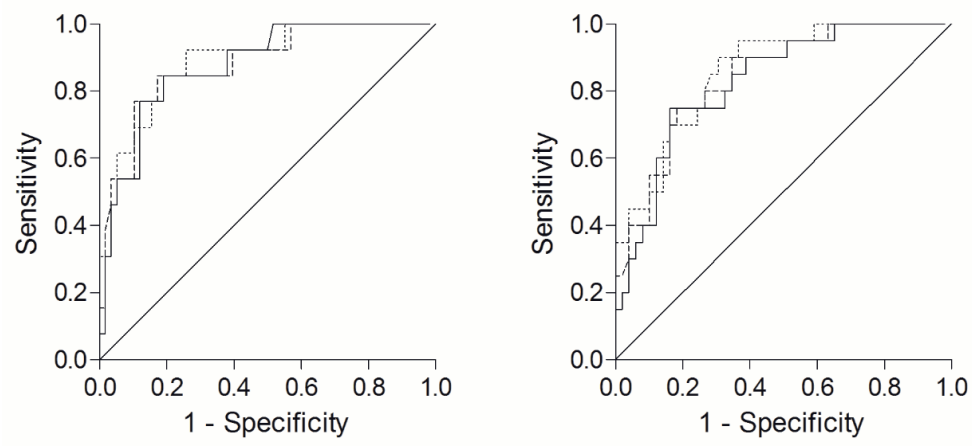

Figure 3 Receiver operator curves for predicting a peak oxygen uptake $\leq 18.2 \mathrm{~mL} \cdot \mathrm{kg}^{-1} \cdot \mathrm{min}^{-1}$ (left graph) and a ventilatory anaerobic threshold $\leq 11.1 \mathrm{~mL} \cdot \mathrm{kg}^{-1} \cdot \mathrm{min}^{-1}$ (right graph) from the oxygen uptake efficiency slope normalized for body mass calculated using all exercise data (-), $90 \%$ of the data (---), and $80 \%$ of the data ( $\cdots)$.

Abbreviations: OUES100. $\mathrm{kg}^{-1}$, OUES90. $\mathrm{kg}^{-1}$, and OUES80. $\mathrm{kg}^{-1}$, oxygen uptake efficiency slope normalized for body mass calculated using all exercise data, $90 \%$ of the data, and $80 \%$ of the data, respectively.

Table 3 Optimal cut-off values for OUES including sensitivity and specificity.

\begin{tabular}{|c|c|c|c|}
\hline Peak oxygen uptake $\leq 18.2 \mathrm{~mL} . \mathrm{kg}^{-1} \cdot \mathrm{min}^{-1}$ & Optimal cut-off & ensitivity & pecificity \\
\hline OUES100.kg ${ }^{-1}$ & 20.6 & 0.846 & 0.810 \\
\hline OUES90.kg-1 & 20.9 & 0.846 & 0.828 \\
\hline OUES80. $\mathrm{kg}^{-1}$ & 19.8 & 0.923 & 0.741 \\
\hline
\end{tabular}

Ventilatory anaerobic threshold $\leq 11.1 \mathrm{~mL} \cdot \mathrm{kg}^{-1} \cdot \mathrm{min}^{-1}$ a Optimal cut-off Sensitivity Specificity

\begin{tabular}{llll}
\hline OUES100. $\mathrm{kg}^{-1}$ & 20.5 & 0.750 & 0.837 \\
OUES90. $\mathrm{kg}^{-1}$ & 20.1 & 0.750 & 0.816 \\
OUES80. $\mathrm{kg}^{-1}$ & 19.3 & 0.900 & 0.694 \\
\hline
\end{tabular}

a: the ventilatory anaerobic threshold was not determinable in two patients (3\%), so in this case $n=69$. Abbreviations: OUES100. $\mathrm{kg}^{-1}$, OUES90. $\mathrm{kg}^{-1}$, and OUES80. $\mathrm{kg}^{-1}$, oxygen uptake efficiency slope normalized for body mass calculated using all exercise data, $90 \%$ of the data, and $80 \%$ of the data, respectively.

\section{Discussion}

This study aimed to investigate the validity of the oxygen uptake efficiency slope as an alternative measure of cardiorespiratory fitness in elderly patients undergoing major elective colorectal surgery. The main results show that the oxygen uptake efficiency slope provides an objective and valid measure of cardiorespiratory fitness in these patients, as indicated by the strong correlations with peak oxygen uptake and the ventilatory anaerobic threshold. Furthermore, its ability to predict cut-off points for peak oxygen uptake and the ventilatory anaerobic threshold might help discriminate patients at higher 
risk of postoperative morbidity at the stage of diagnosis and contemplation of elective surgery.

Theoretically, the oxygen uptake efficiency slope should be relatively insensitive to the duration of the cardiopulmonary exercise test, which is an essential characteristic when a patient is either unwilling or unable to deliver a maximal effort. Most studies found no significant differences between maximal and submaximal oxygen uptake efficiency slope values, ${ }^{19,21,22,24,25,28}$ whereas other studies reported small differences. ${ }^{14,18,20,26}$ The current study found that within patients, OUES100 values were slightly but statistically significant lower than OUES90 and OUES80 values, whereas OUES90 and OUES80 values did not differ within the study groups. These small differences might be explained by the fact that the oxygen uptake efficiency slope profile throughout the cardiopulmonary exercise test demonstrates an ' $S$ '-shaped or sigmoid curve, with 1) an initial slow rising phase, 2) a subsequent accelerated rising phase followed by a decelerating rising phase, and 3) a final slow rising phase at maximal exercise. ${ }^{38}$ As a consequence, Niemijer et al..$^{38}$ recommended that oxygen uptake efficiency slope values should be interpreted with caution when patients do not reach the ventilatory anaerobic threshold or when an oxygen uptake plateau is present. We performed a post-hoc analysis in patients with a difference of $>200$ between OUES100 and OUES 80 values in order to elucidate the statistically significant differences between the OUES100 and the OUES90, and between the OUES100 and the OUES80. The two patients (both men, 72.3 and 75.9 years of age) who had a difference of $>200$ between OUES100 and OUES80 values, demonstrated a consistent levelling-off of oxygen uptake during the last part of the cardiopulmonary exercise test, without showing a clear oxygen uptake plateau (see Figure 4A.2 in Appendix 4.2 for an example). After excluding these two patients, statistically significant differences were found between the OUES100, OUES90, and OUES80 were no longer present. Additionally, the small differences between the OUES100 and OUES90, and between the OUES100 and the OUES80 do not seem clinically relevant, as OUES100, OUES90, and OUES80 values all correlated strongly with each other, as well as with peak oxygen uptake and the ventilatory anaerobic threshold. This strong correlation between the oxygen uptake efficiency slope and peak oxygen uptake is in agreement with other studies ${ }^{\text {e.g.,14,18-30 }}$ and indicates that the oxygen uptake efficiency slope is an objective measure of cardiorespiratory fitness.

We defined patients being 'fit' or 'unfit' based on the recently published thresholds of peak oxygen uptake $\leq 18.2 \mathrm{~mL} \cdot \mathrm{kg}^{-1} \cdot \mathrm{min}^{-1}$ and a ventilatory anaerobic threshold $\leq 11.1$ $\mathrm{mL} . \mathrm{kg}^{-1} \cdot \mathrm{min}^{-1}{ }^{12}$ Receiver operating characteristic analysis showed that the oxygen uptake efficiency slope normalized for body mass (OUES. $\mathrm{kg}^{-1}$ ) is a sensitive and specific predictor of peak oxygen uptake $\leq 18.2 \mathrm{~mL} \cdot \mathrm{kg}^{-1} \cdot \mathrm{min}^{-1}$ and a ventilatory anaerobic threshold $\leq 11.1$ $\mathrm{mL} . \mathrm{kg}^{-1} \cdot \mathrm{min}^{-1}$. Although these thresholds for risk are derived from a different patient population and study, the current study indicates that the oxygen uptake efficiency slope 
might possess sufficient discriminative power to distinguish between patients with a higher likelihood (ventilatory anaerobic threshold $\leq 11.1 \mathrm{~mL} \cdot \mathrm{kg}^{-1} \cdot \mathrm{min}^{-1}$ ) and patients with a lower likelihood (ventilatory anaerobic threshold $>11.1 \mathrm{~mL} . \mathrm{kg}^{-1} \cdot \mathrm{min}^{-1}$ ) of in-hospital morbidity after major colorectal surgery (Table 2).

Evaluating physiological parameters of cardiorespiratory fitness, obtained at specific time points during the cardiopulmonary exercise test (e.g., peak oxygen uptake or the ventilatory anaerobic threshold) remains the most common clinical test for preoperative risk stratification in major elective abdominal surgery. The measurement of peak oxygen uptake, however, can be strongly influenced by the patient's motivation to deliver a maximal effort, and might be invalid in patients whose performance may be limited because of pain or fatigue rather than cardiorespiratory deficiency. In case of a submaximal effort due to premature cessation of the cardiopulmonary exercise test, the oxygen uptake occurring at the ventilatory anaerobic threshold may represent a submaximal indicator of cardiorespiratory fitness. Although the inter-observer variability in a preoperatively measured ventilatory anaerobic threshold has been reported to be acceptable for experienced clinicians, ${ }^{39}$ it cannot always be determined, and its values has been reported to depend on the choice of ergometer, exercise protocol, and method of detection. ${ }^{40-43}$ Additionally, only focusing on physiological parameters at specific time points (e.g., peak oxygen uptake, ventilatory anaerobic threshold, and the ventilatory equivalent for carbon dioxide at the ventilatory anaerobic threshold) leads to the loss of a significant amount of data generated during a cardiopulmonary exercise test that might better reflect the continuous, dynamic cardiopulmonary responses throughout the test. Due to the aforementioned limitations of peak oxygen uptake and the ventilatory anaerobic threshold, it would be of interest to investigate the clinical usefulness of alternative objective (sub)maximal exercise parameters that incorporate cardiopulmonary response profiles for the assessment of cardiorespiratory fitness and prognosis in patients undergoing major surgery. The oxygen uptake efficiency slope gives an estimation of the efficiency of the minute ventilation with respect to the oxygen uptake throughout the cardiopulmonary exercise test by providing a log-transformed response profile indicative of cardiorespiratory function during exercise. Without significant lung disease, the oxygen uptake efficiency slope is indicative of cardiovascular function throughout exercise, in which higher oxygen uptake efficiency slope values indicate a more efficient oxygen uptake. As such, the oxygen uptake efficiency slope might reflect the resilience of a patient to meet the increased metabolic demands from surgical stress.

This is the first study that investigated the validity of the oxygen uptake efficiency slope in patients undergoing major elective colorectal surgery. Although oxygen uptake efficiency slope values were found to be strongly correlated with peak oxygen uptake and the ventilatory anaerobic threshold, the small intra-individual differences in maximal 
and submaximal oxygen uptake efficiency slope values might limit the clinical utility of this parameter; this requires further research. Future research should also address the prognostic value of the oxygen uptake efficiency slope for different postoperative outcomes in major abdominal surgery, as only one study ${ }^{32}$ evaluated the predictive value of the oxygen uptake efficiency slope; this focused on mortality. From the latter study, it is unclear whether the authors normalized absolute oxygen uptake efficiency slope values for body mass, which we believe is necessary, just as with peak oxygen uptake and the ventilatory anaerobic threshold. As already pointed out by Niemijer et al., ${ }^{38}$ it remains debatable whether oxygen uptake efficiency slope values should be calculated after careful visual inspection of oxygen uptake data, in order to use oxygen uptake and minute ventilation data up to the oxygen uptake plateau, or up to the point where a consistent levelling-off of oxygen uptake is observed, even without showing a clear oxygen uptake plateau.

The current study has several limitations. Firstly, due to the retrospective evaluation of the tests, there is a possibility of bias. Secondly, our data are derived from a single centre and therefore may not apply to patients from other centres. Thirdly, the number of patients $(n=71)$ is relatively small and we did not perform a prospective sample size calculation. These issues affect statistical analysis and generalizability, as does the fact that the thresholds for risk of the peak oxygen uptake and ventilatory anaerobic threshold were based on the cut-off points described in another study and patient population. ${ }^{12}$ Finally, no direct clinical outcomes were presented, which might hinder clinicians from estimating more exactly the seriousness of illness of the population at hand. However, as our aim was to investigate the validity of the oxygen uptake efficiency slope as an effortindependent indicator of cardiorespiratory fitness, we do not consider the latter issue to be crucial.

In conclusion, the strong correlations with peak oxygen uptake and the ventilatory anaerobic threshold indicate that the oxygen uptake efficiency slope provides an objective and valid measure of cardiorespiratory fitness in elderly patients scheduled for major colorectal surgery, and its ability to predict cut-off points for peak oxygen uptake and the ventilatory anaerobic threshold indicates that it might help discriminate patients at higher risk of postoperative morbidity. Future research should investigate the prognostic value of the oxygen uptake efficiency slope for postoperative outcomes in this group. 


\section{References}

1. Older P, Smith R, Courtney P, Hone R. Preoperative evaluation of cardiac failure and ischemia in elderly patients by cardiopulmonary exercise testing. Chest 1993; 104: 701-4.

2. Older P, Hall A, Hader R. Cardiopulmonary exercise testing as a screening test for perioperative management of major surgery in the elderly. Chest 1999; 116: 355-62.

3. Wilson RJ, Davies S, Yates D, Redman J, Stone M. Impaired functional capacity is associated with all-cause mortality after major elective intra-abdominal surgery. British Journal of Anaesthesia 2010; 105: 297-303.

4. Snowden CP, Prentis JM, Anderson HL, et al. Submaximal cardiopulmonary exercise testing predicts complications and hospital length of stay in patients undergoing major elective surgery. Annals of Surgery 2010; 251: 535-41.

5. Junejo MA, Mason JM, Sheen AJ, et al. Cardiopulmonary exercise testing for preoperative risk assessment before hepatic resection. British Journal of Surgery 2012; 99: 1097-104.

6. Chandrabalan VV, McMillan DC, Carter R, et al. Preoperative cardiopulmonary exercise testing predicts adverse post-operative events and non-progression to adjuvant therapy after major pancreatic surgery. HPB 2013; 15: 899-907.

7. Junejo MA, Mason JM, Sheen AJ, et al. Cardiopulmonary exercise testing for preoperative risk assessment before pancreaticoduodenectomy for cancer. Annals of Surgical Oncology 2014; 21: 1929-36.

8. West MA, Lythgoe D, Barben CP, et al. Cardiopulmonary exercise variables are associated with postoperative morbidity after major colonic surgery: a prospective blinded observational study. British Journal of Anaesthesia 2014; 112: 665-71.

9. West MA, Parry MG, Lythgoe D, et al. Cardiopulmonary exercise testing for the prediction of morbidity risk after rectal cancer surgery. British Journal of Surgery 2014; 101: 1166-72.

10. Ulyett S, Wiggans MG, Bowles MJ, et al. Clinical assessment before hepatectomy identifies highrisk patients. Journal of Surgical Research 2015; 198: 87-92.

11. Kasivisvanathan R, Abbassi-Ghadi N, McLeod AD, et al. Cardiopulmonary exercise testing for predicting postoperative morbidity in patients undergoing hepatic resection surgery. $H P B$ 2015; 17: 637-43.

12. West MA, Asher R, Browning M, et al; Perioperative Exercise Testing and Training Society. Validation of preoperative cardiopulmonary exercise testing-derived variables to predict inhospital morbidity after major colorectal surgery. British Journal of Surgery 2016; 103: 744-52.

13. Moran J, Wilson F, Guinan E, McCormick P, Hussey J, Moriarty J. Role of cardiopulmonary exercise testing as a risk-assessment method in patients undergoing intra-abdominal surgery: a systematic review. British Journal of Anaesthesia 2016; 116: 177-91.

14. Davies LC, Wensel R, Georgiadou P, et al. Enhanced prognostic value from cardiopulmonary exercise testing in chronic heart failure by non-linear analysis: oxygen uptake efficiency slope. European Heart Journal 2006; 27: 684-90. 
15. Myers J, Arena R, Dewey F, et al. A cardiopulmonary exercise testing score for predicting outcomes in patients with heart failure. American Heart Journal 2008; 156: 1177-83.

16. Coeckelberghs E, Buys R, Goetschalckx K, Cornelissen VA, Vanhees L. Prognostic value of the oxygen uptake efficiency slope and other exercise variables in patients with coronary artery disease. European Journal of Preventive Cardiology 2016; 23: 237-44.

17. Lin YS, Huang HY, Lin WH, et al. Oxygen uptake efficiency slope predicts major cardiac events in patients with end-stage heart failure. Transplantation Proceedings 2016; 48: 956-8.

18. Baba R, Nagashima M, Goto $M$, et al. Oxygen uptake efficiency slope: a new index of cardiorespiratory functional reserve derived from the relation between oxygen uptake and minute ventilation during incremental exercise. Journal of the American College of Cardiology 1996; 28: 1567-72.

19. Baba R, Tsuyuki K, Kimura Y, et al. Oxygen uptake efficiency slope as a useful measure of cardiorespiratory functional reserve in adult cardiac patients. European Journal of Applied Physiology 1999; 80: 397-401.

20. Hollenberg M, Tager IB. Oxygen uptake efficiency slope: an index of exercise performance and cardiopulmonary reserve requiring only submaximal exercise. Journal of the American College of Cardiology 2000; 36: 194-201.

21. Marinov B, Kostianev S. Exercise performance and oxygen uptake efficiency slope in obese children performing standardized exercise. Acta Physiologica et Pharmacologica Bulgarica 2003; 27: 1-6.

22. van Laethem C, Bartunek J, Goethals M, Nellens P, Andries E, Vanderheyden M. Oxygen uptake efficiency slope, a new submaximal parameter in evaluating exercise capacity in chronic heart failure patients. American Heart Journal 2005; 149: 175-80.

23. van de Veire NR, van Laethem C, Philippé J, et al. VE/VCO2 slope and oxygen uptake efficiency slope in patients with coronary artery disease and intermediate peak VO2. European Journal of Cardiovascular Prevention and Rehabilitation 2006; 13: 916-23.

24. Pogliaghi S, Dussin E, Tarperi C, Cevese A, Schena F. Calculation of oxygen uptake efficiency slope based on heart rate reserve end-points in healthy elderly subjects. European Journal of Applied Physiology 2007; 101: 691-6.

25. Akkerman M, van Brussel M, Bongers BC, Hulzebos EH, Helders PJ, Takken T. Oxygen uptake efficiency slope in healthy children. Pediatric Exercise Science 2010; 22: 431-41.

26. Gruet M, Brisswalter J, Mely L, Vallier JM. Clinical utility of the oxygen uptake efficiency slope in cystic fibrosis patients. Journal of Cystic Fibrosis 2010; 9: 307-13.

27. Buys R, Cornelissen V, van de Bruaene A, et al. Measures of exercise capacity in adults with congenital heart disease. International Journal of Cardiology 2011; 153: 26-30.

28. Bongers BC, Hulzebos HJ, Blank AC, van Brussel M, Takken T. The oxygen uptake efficiency slope in children with congenital heart disease: construct and group validity. European Journal of Cardiovascular Prevention and Rehabilitation 2011; 18: 384-92. 
29. Müller Pde T, Viegas CA, Patusco LA. Muscle strength as a determinant of oxygen uptake efficiency and maximal metabolic response in patients with mild-to-moderate COPD. Jornal Brasileiro de Pneumologia 2012; 38: 541-9.

30. Heine M, Verschuren $\mathrm{O}$, Kwakkel G. Validity of oxygen uptake efficiency slope in patients with multiple sclerosis. Journal of Rehabilitation Medicine 2014; 46: 656-61.

31. Phypers BJ, Robiony-Rogers D, Pickering RM, Garden AL. Test-retest reliability of the oxygen uptake efficiency slope in surgical patients. Anaesthesia 2011; 66: 659-66.

32. Colson M, Baglin J, Bolsin S, Grocott MP. Cardiopulmonary exercise testing predicts 5 yr survival after major surgery. British Journal of Anaesthesia 2012; 109: 735-41.

33. Myers J, Do D, Herbert W, RibisI P, Froelicher VF. A nomogram to predict exercise capacity from a specific activity questionnaire and clinical data. American Journal of Cardiology 1994; 73: 591-6.

34. American Thoracic Society; American College of Chest Physicians. ATS/ACCP statement on cardiopulmonary exercise testing. American Journal of Respiratory and Critical Care Medicine 2003; 167: 211-77.

35. Lamarra N, Whipp BJ, Ward SA, Wasserman K. Effect of interbreath fluctuations on characterizing exercise gas exchange kinetics. Journal of Applied Physiology 1987; 62: 2003-12.

36. Beaver WL, Wasserman K, Whipp BJ. A new method for detecting anaerobic threshold by gas exchange. Journal of Applied Physiology 1986; 60: 2020-7.

37. Wasserman K, Hansen JE, Sue DY, Stringer WW, Whipp BJ. Principles of exercise testing and interpretation: including pathophysiology and clinical applications. Philadelphia: Lippincott Williams \& Wilkins, 2005.

38. Niemeijer VM, van 't Veer M, Schep G, Spee RF, Hoogeveen A, Kemps HM. Causes of nonlinearity of the oxygen uptake efficiency slope: a prospective study in patients with chronic heart failure. European Journal of Preventive Cardiology 2014; 21: 347-53.

39. Sinclair RC, Danjoux GR, Goodridge V, Batterham AM. Determination of the anaerobic threshold in the preoperative assessment clinic: inter-observer measurement error. Anaesthesia 2009; 64: 1192-5.

40. Shimizu M, Myers J, Buchanan N, et al. The ventilatory threshold: method, protocol, and evaluator agreement. American Heart Journal 1991; 122: 509-16.

41. Meyer K, Hajric R, Westbrook S, et al. Ventilatory and lactate threshold determinations in healthy normals and cardiac patients: methodological problems. European Journal of Applied Physiology and Occupational Physiology 1996; 72: 387-93.

42. Vanhees L, Lefevre J, Philippaerts R, et al. How to assess physical activity? How to assess physical fitness? European Journal of Cardiovascular Prevention and Rehabilitation 2005; 12: 102-14.

43. Akkerman M, van Brussel M, Hulzebos E, Vanhees L, Helders PJ, Takken T. The oxygen uptake efficiency slope: what do we know? Journal of Cardiopulmonary Rehabilitation and Prevention 2010; 30: 357-73. 


\section{Appendix}

\section{Appendix 4.I}

Table 4A.1 Correlation between oxygen uptake efficiency slope values at different exercise intensities, peak oxygen uptake, and the ventilatory anaerobic threshold.

\begin{tabular}{|c|c|c|c|}
\hline Parameter & OUES100 & OUES90 & OUES80 \\
\hline Peak oxygen uptake $\left(\mathrm{mL} \cdot \mathrm{min}^{-1}\right)$ & $r=0.816 p<0.001$ & $r=0.821 p<0.001$ & $r=0.825 p<0.001$ \\
\hline $\begin{array}{l}\text { Ventilatory anaerobic threshold }(\mathrm{mL} \text {. } \\
\left.\min ^{-1}\right)^{\mathrm{a}}\end{array}$ & $r=0.793 p<0.001$ & $r=0.800 p<0.001$ & $r=0.805 p<0.001$ \\
\hline OUES100 & - & $r=0.991 p<0.001$ & $r=0.976 p<0.001$ \\
\hline OUES90 & $r=0.991 p<0.001$ & - & $r=0.992 p<0.001$ \\
\hline OUES80 & $r=0.976 p<0.001$ & $r=0.992 p<0.001$ & - \\
\hline Parameter & OUES100.kg ${ }^{-1}$ & OUES90.kg ${ }^{-1}$ & OUES80. $\mathrm{kg}^{-1}$ \\
\hline Peak oxygen uptake $\left(\mathrm{mL} \cdot \mathrm{kg}^{-1} \cdot \mathrm{min}^{-1}\right)$ & $r=0.763 p<0.001$ & $r=0.776 p<0.001$ & $r=0.780 p<0.001$ \\
\hline $\begin{array}{l}\text { Ventilatory anaerobic threshold }(\mathrm{mL} \text {. } \\
\left.\mathrm{kg}^{-1} \cdot \mathrm{min}^{-1}\right)^{\mathrm{a}, \mathrm{b}}\end{array}$ & $r=0.640 p<0.001$ & $r=0.671 p<0.001$ & $r=0.700 p<0.001$ \\
\hline OUES100.kg & - & $r=0.989 p<0.001$ & $r=0.970 p<0.001$ \\
\hline OUES90.kg-1 & $r=0.989 p<0.001$ & - & $r=0.989 p<0.001$ \\
\hline OUES80.kg-1 & $r=0.970 p<0.001$ & $r=0.989 p<0.001$ & - \\
\hline \multicolumn{4}{|c|}{$\begin{array}{l}\text { a: the ventilatory anaerobic threshold was not determinable in two patients (3\%), so in this case } n=69 \text {. } \\
\text { b: Spearman's rho. } \\
\text { Abbreviations: OUES100, OUES90, and OUES80, oxygen uptake efficiency slope calculated using all exercise } \\
\text { data, } 90 \% \text { of the data, and } 80 \% \text { of the data, respectively; OUES100. } \mathrm{kg}^{-1} \text {, OUES90. } \mathrm{kg}^{-1} \text {, and OUES80.kg } \text { O }^{-1} \text { oxygen } \\
\text { uptake efficiency slope normalized for body mass calculated using all exercise data, } 90 \% \text { of the data, and } 80 \% \\
\text { of the data, respectively. }\end{array}$} \\
\hline
\end{tabular}




\section{Appendix 4.2}

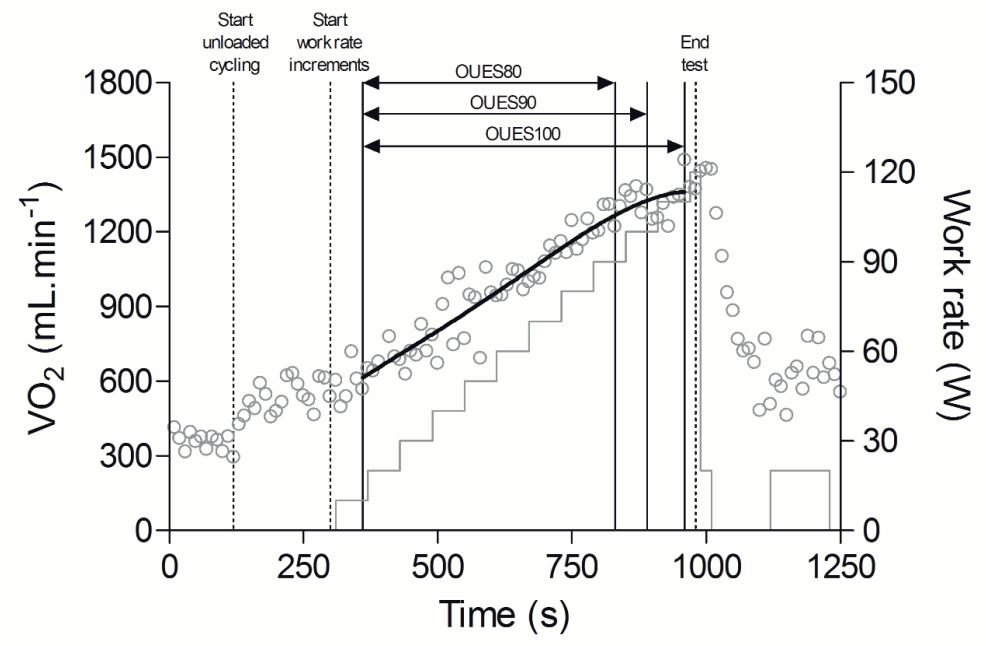

Figure 4A.2 Oxygen uptake and work rate throughout the cardiopulmonary exercise test in a 72-year-old man. In this case, OUES100 is $1478.7\left(R^{2}=0.937\right)$, OUES90 is $1623.8\left(R^{2}=0.956\right)$, and OUES80 is $1680.0\left(R^{2}=0.945\right)$. The solid line depicts the leveling-off of oxygen uptake during the last part of the cardiopulmonary exercise test, without showing a clear oxygen uptake plateau.

Abbreviations: OUES100, OUES90, and OUES80, oxygen uptake efficiency slope calculated using all exercise data, $90 \%$ of the data, and $80 \%$ of the data, respectively; $\mathrm{VO}_{2}=$ oxygen uptake. 



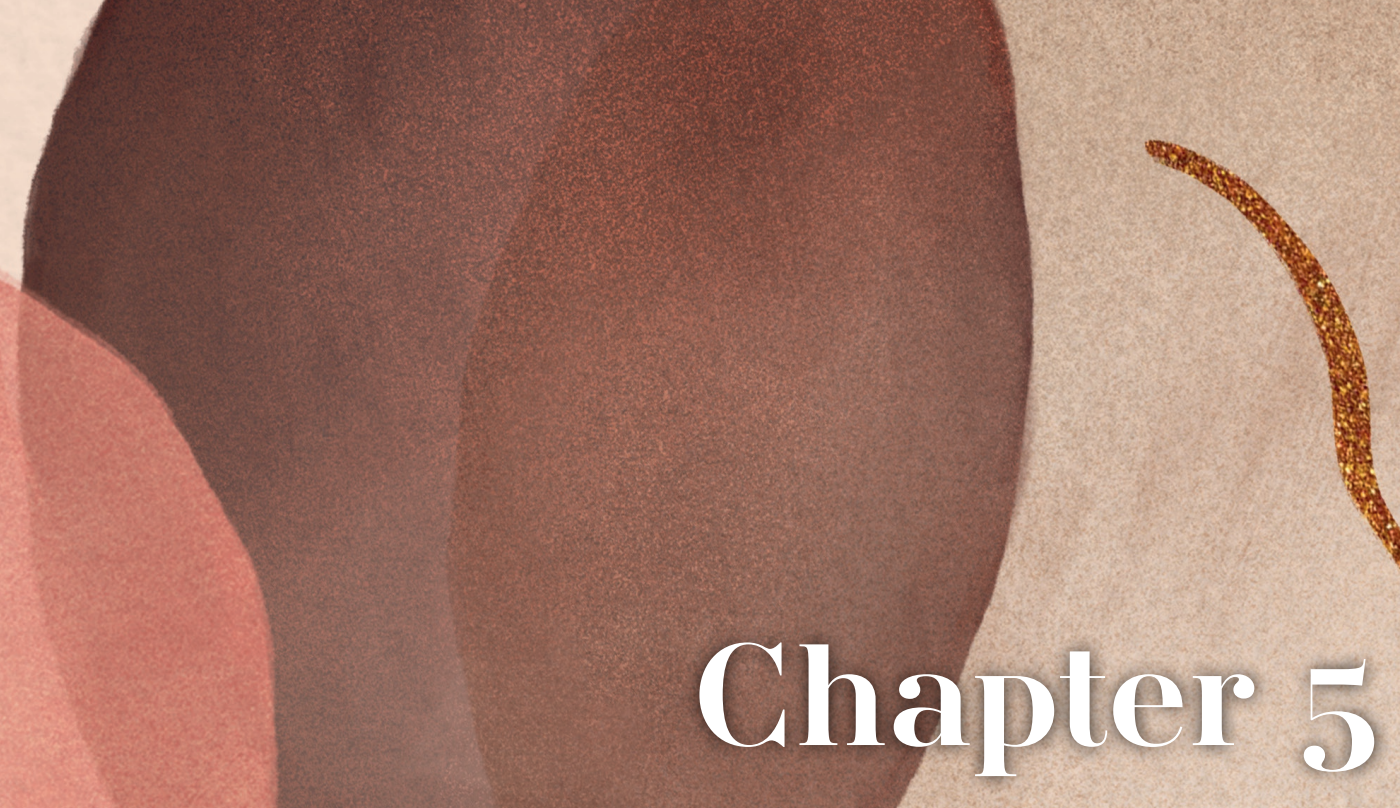

The association between preoperative body composilion and aerobic fituess in patients scheduled for colorectal surgery

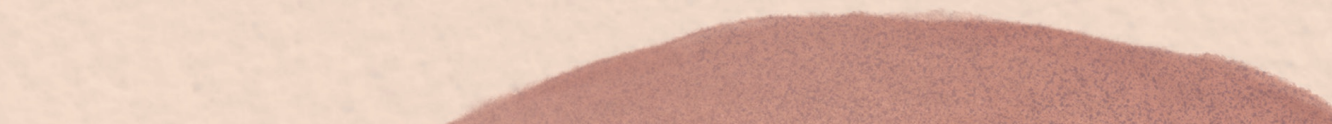

Annefleur E.M. Berkel ${ }^{\star}$, Laura van Wijk* , David P.J. van Dijk, Sanne Prins, - Jobvan der Palen, Nico L.U. van Meeteren, Steven W.M. Olde Damink, Joost M. Klaase, Bart C. Bongers

Colorectal Dis, 2021;00:1-9. https://doi.org/10.1111/codi.15941

${ }^{\star}$ Shared first authorship 


\section{Abstract}

Background: Though cardiopulmonary exercise testing (CPET) is considered the gold standard, the preoperative abdominal computed tomography (CT) scan might also provide information concerning preoperative aerobic fitness. This study aimed to investigate the association between preoperative CT-scan derived body composition variables and CPET variables of aerobic fitness in older patients scheduled for colorectal surgery.

Methods: CT-images at level L3 were analyzed for skeletal muscle mass, skeletal muscle radiation attenuation, visceral adipose tissue (VAT) mass, and subcutaneous adipose tissue mass. Regression analyses were performed to investigate the relation between CT-scan derived body composition variables, CPET-derived aerobic fitness, and other preoperative patient-related variables. Multivariable logistic regression analysis was performed to predict an anaerobic threshold (AT) $\leq 11.1 \mathrm{~mL} / \mathrm{kg} / \mathrm{min}$.

Results: Data of 78 patients (45 males, mean (SD) age 74.5 (6.4) years) were analyzed. A correlation coefficient of 0.55 was observed between absolute AT and skeletal muscle mass index. Absolute AT (R2 of 51.1\%) was lower in patients with a lower skeletal muscle mass index, together with higher age, lower body mass, and higher American Society of Anesthesiologists (ASA) score. Higher ASA score (odds ratio [OR] 5.64, 95\% confidence interval $[\mathrm{Cl}] 1.15-27.69, \mathrm{P}=0.033$ ) and higher VAT mass ( $\mathrm{OR} 1.02,95 \% \mathrm{Cl} 1.00-1.03, \mathrm{P}=0.036$ ) were associated with an increased risk of a relative $A T \leq 11.1 \mathrm{~mL} / \mathrm{kg} / \mathrm{min}$.

Conclusion: Body composition variables from the preoperative CT-scan were moderately associated with preoperative CPET-derived aerobic fitness. Higher ASA score and higher VAT mass were associated with an increased risk of a relative AT $\leq 11.1 \mathrm{~mL} / \mathrm{kg} / \mathrm{min}$. 


\section{Introduction}

Colorectal cancer is the third most common type of cancer. ${ }^{1}$ After resection for colon or rectal carcinoma, $15 \%$ and $20 \%$ of the patients respectively have a complicated course within 30 days after surgery, which might lead to a prolonged hospital stay of $>14$ days or even mortality. ${ }^{2}$ Reducing complications will result in considerable cost savings. ${ }^{3}$ Preoperative risk assessment might identify patients at high risk of postoperative complications; these patients may benefit from preoperative preventive interventions (prehabilitation). ${ }^{4,5}$

Cardiopulmonary exercise testing (CPET) is increasingly utilized for risk assessment before major surgery to evaluate the risk of adverse perioperative events. ${ }^{5} \mathrm{CPET}$ is an objective and precise method of evaluating a patient's preoperative aerobic fitness. In general, patients with a lower oxygen uptake at the anaerobic threshold (AT) and/or a lower oxygen uptake at peak exercise $\left(\mathrm{VO}_{\text {2peak }}\right)$ have an increased risk of postoperative complications. ${ }^{6-9}$ Despite its usefulness in perioperative medicine, CPET is relatively expensive, time-consuming, and requires well-trained personnel for an adequate interpretation of its results.

For preoperative risk assessment, measurements of body composition using the routinely performed abdominal computed tomography (CT) scan is increasingly gaining ground. Sarcopenia, ${ }^{10}$ a low skeletal muscle radiation attenuation (SM-RA), ${ }^{11,12}$ and a high visceral adipose tissue (VAT) mass ${ }^{13,14}$ have all been reported to be associated with poor clinical outcome following abdominal surgery. Furthermore, Boo and others ${ }^{15}$ demonstrated that skeletal muscle mass is closely associated with aerobic fitness (the AT and $\mathrm{VO}_{2 \text { peak }}$ ) in community-dwelling elderly men, while a recent study of West and others ${ }^{16}$ in patients undergoing hepatopancreatobiliary surgery reported that SM-RA and not skeletal muscle mass (assessed by a preoperative CT-scan) were associated with aerobic fitness (assessed with preoperative CPET).

Although CPET is the gold standard to assess aerobic fitness, it would be of interest for time and cost savings to investigate whether the routinely performed preoperative abdominal CT-scan can (assist to pre)select unfit patients. Therefore, the aim of this study was to preoperatively investigate the association between body composition variables derived from the abdominal CT-scan and CPET variables of aerobic fitness in patients scheduled for colorectal surgery. 


\section{Materials and methods}

\section{Participants}

Data from all patients $\geq 60$ years with colorectal cancer or dysplasia planned for elective colorectal resection at the hospital Medisch Spectrum Twente, with a veterans-specific activity questionnaire (VSAQ) score $\leq 7$ metabolic equivalents of task (METs) and who underwent a preoperative abdominal CT-scan and preoperative CPET between February 2013 and May 2017 were included. The VSAQ is a brief self-administered questionnaire to estimate aerobic fitness, in which a score $\leq 7$ METs was used to preselect those patients with a low perceived aerobic fitness. ${ }^{17}$ These formed the study data and were retrospectively analyzed after this period. Ethical approval for the study protocol (registration number P13-18) was provided by the Medical Ethics Committee Twente (Dr. J.F.F. Lekkerkerker, clinical pharmacologist, chairman) in September 2013. Patients were excluded if the time between CPET and CT was $>60$ days, or when acute surgery of the tumor was necessary.

\section{Computed tomography scan}

A single slice of each patient's routinely performed preoperative abdominal CT-scan was selected at the level of the third lumbar vertebra (L3) on which both transverse processes were visible. CT-scans were all screened for their quality. Patients with a CT-scan of poor quality (e.g., large radiation artefacts, low-dose) were excluded from analysis. Scans were analyzed using sliceOmatic 5 (TomoVision, Magog, Canada) software for Microsoft Windows ${ }^{\circ}$. The cross-sectional areas $\left(\mathrm{cm}^{2}\right)$ of skeletal muscle tissue, VAT, and subcutaneous adipose tissue (SAT) were colored automatically, and manually corrected if necessary by two trained and blinded researchers (LVW and checked by DVD, both blinded for CPET analyses). Skeletal muscle tissue, VAT, and SAT areas were normalized for the patient's body height to calculate the $\mathrm{L} 3$ index in $\mathrm{cm}^{2} / \mathrm{m}^{2}$. The SM-RA was assessed by calculating the average Hounsfield Units (HU) value of skeletal muscle mass. Low SM-RA is associated with increased inter- and intramyocellular fat (myosteatosis). ${ }^{18}$

\section{Cardiopulmonary exercise testing}

As part of the study protocol an incremental CPET was performed by patients preoperatively under controlled conditions at the lung function department, using a calibrated electronically braked cycle ergometer in upright position (Ergoline, Ergoselect 100, Bitz, Germany). The following standardized pre-test instructions were given to the patients: 1) consume the last (light) meal at least two hours before exercise testing, 2) adhere to usual use of medication, and 3) wear comfortable sporting clothes and shoes. CPET comprised a two-minute resting phase to assess baseline cardiopulmonary values, followed by three minutes of unloaded cycling (warm-up), where after the work rate was progressively increased with constant increments of 5,10 , or $15 \mathrm{~W} / \mathrm{min}$, depending on the patient's subjective physical fitness level and aimed at reaching a maximal effort within 
eight to twelve minutes. Throughout CPET, patients had to maintain a pedalling frequency between 60 and 80 revolutions/min. The protocol continued until the patient's pedalling frequency fell definitely $<60$ revolutions/min, despite strong verbal encouragement. After test termination, the patient completed a five minute-recovery phase of unloaded cycling (cool-down).

During CPET, patients breathed through a facemask (Hans Rudolph, Kansas City, MO, USA) connected to a Triple V volume transducer to calculate breath-by-breath minute ventilation (VE), oxygen uptake $\left(\mathrm{VO}_{2}\right)$, carbon dioxide production $\left(\mathrm{VCO}_{2}\right)$, and the respiratory exchange ratio (RER) averaged at ten-second intervals (Oxycon Pro, Jaeger, Hoechberg, Germany). Flow-volume (three-litre syringe) and gas calibration (ambient air and a gas mixture of $16 \%$ oxygen and $5 \%$ carbon dioxide) were performed manually before each test. Heart rate (HR), twelve-lead electrocardiography, blood pressure, and pulse oximetry were continuously monitored.

CPET data were interpreted by a trained and experienced clinical exercise physiologist (BB, blinded for CT-scan analyses). The highest HR achieved during the CPET was defined as $\mathrm{HR}_{\text {peak }}$. Data from other outcome variables were averaged over 30 seconds of exercise. $\mathrm{VO}_{2 \text { peak }}$ values were considered valid when at least one of the following criteria was met: a heart rate at peak exercise $>95 \%$ of predicted (predicted peak heart rate [beats $/ \mathrm{min}$ ] $=208-0.7 \times$ age [years]) or a respiratory exchange ratio at peak exercise $>1.10$. The AT was defined as the point at which the ventilatory equivalent for oxygen and the partial end-tidal oxygen tension reached a minimum and thereafter began to rise in a consistent manner, coinciding with an unchanged ventilatory equivalent for carbon dioxide and partial end-tidal carbon dioxide tension. ${ }^{19}$ In case this ventilatory equivalents method provided uncertain results, the V-slope method was used to estimate the AT (the point at which the linear slope of the relation between the $\mathrm{VCO}_{2}$ and $\mathrm{VO}_{2}$ changed)..$^{20}$ Finally, the oxygen uptake efficiency slope (OUES), which provides a valid objective effortindependent measure of aerobic fitness in elderly patients scheduled for major colorectal surgery was calculated. ${ }^{21}$ Absolute $\mathrm{VO}_{2 \text { peak }}, \mathrm{AT}$, and OUES values were normalized for body mass as well.

\section{Patient characteristics and outcome measures}

Baseline patient characteristics included sex, age, body height, body mass, body mass index (BMI), smoking status, use of beta-blocker, METs score on the veterans-specific activity questionnaire, clinical signs of metastasis, American Society of Anaesthesiologists (ASA) score (I-IV), and Charlson comorbidity index (divided in three groups: 0, 1, and 2+).

Body composition and aerobic fitness outcomes were reported separately for men and women, as it is known that values significantly differ between sexes. 


\section{Statistical analysis}

Data were analyzed with the Statistical Package for the Social Sciences for Windows (version 23.0; IBM, SPSS Inc., Chicago, IL, USA). Continuous data were presented as mean and standard deviation (SD), or as median and interquartile range (IQR), where appropriate. Categorical data were summarized by frequency and percentage. Pearson or Spearman correlation coefficients were calculated to examine univariable associations between continuous variables, depending upon the distribution of the variables. To investigate the univariable association between a continuous variable (e.g., AT) and a categorical variable, the one-way ANOVA, the independent samples t-test, or the Mann Whitney $U$ test, as appropriate, was used. Univariable associations with a $P<0.10$ were included in the multivariable analysis. For predicting continuous outcomes, linear regression analyses (method: enter) were performed to investigate the association between continuous CPET variables (dependent variable, e.g., AT) and preoperative independent variables.

A multivariable logistic regression analysis was performed to predict whether a patient had a relative $A T \leq 11.1 \mathrm{~mL} / \mathrm{kg} / \mathrm{min}$. Preoperative variables were tested for their association with a relative $A T \leq 11.1 \mathrm{~mL} / \mathrm{kg} / \mathrm{min}(P<0.10)$, using the t-test, Mann Whitney $U$ test, Fisher's exact test, or $\mathrm{Chi}^{2}$ test, as appropriate. A logistic regression model was performed to select which of the remaining variables were significant in a forward stepwise procedure $(P$ in $0.10, P$ out 0.15$)$. In case of multicollinearity between variables, the variable that produced the best model fit (based on the -2 log likelihood) was included in the model. With the final selected significant variables, a new logistic regression model was made (method: enter) to utilize the maximum number of observations. Receiver operator curve (ROC) analysis was used to assess the independent ability of predictive variables to discriminate between patients with and without a relative AT $\leq 11.1 \mathrm{~mL} / \mathrm{kg} / \mathrm{min}$; this AT cut-off was based on the work by West and others ${ }^{8}$ in patients undergoing major colorectal surgery. The optimal cut-off point from the ROC analysis was based on our preference to have primarily a high sensitivity (with a reasonable specificity), as we aim to detect almost all high-risk patients that might benefit from a preoperative intervention (e.g., prehabilitation). A $P<0.05$ was considered statistically significant.

\section{Results}

\section{Patients}

Between February 2013 and May 2017, a total of 371 potential patients $\geq 60$ years with a colorectal tumor were assessed for eligibility. Of these patients, 189 (50.9\%) had a VSAQ score $\leq 7$ METs, of which 91 patients (48.1\%) underwent a preoperative CPET. Of these 91 patients, 13 patients were excluded: in two patients $(2.2 \%)$ skeletal muscle radiation attenuation could not be measured using their CT-scan; in nine patients (9.9\%) 
raw preoperative CPET data was not available; and in two patients (2.2\%) the AT and $\mathrm{VO}_{2 \text { peak }}$ could not be determined due to a poor effort at the CPET (invalid test). Patient characteristics of the remaining 78 patients ( 45 males and 33 females, mean age $74.5 \pm 6.4$ SD years, range 61.5 to 90.3 years) are presented in Table 1.

All 78 patients performed the CPET without any complications or adverse events during or after the test. The AT was undeterminable in two (2.6\%) patients, while they attained a valid $\mathrm{VO}_{2 \text { peak }}$. Normalized for body mass, mean $\pm \mathrm{SD}$ values of $\mathrm{VO}_{2 \text { peak }}$ and AT were $15.6 \pm 3.7$ $\mathrm{mL} / \mathrm{kg} / \mathrm{min}$ and $10.6 \pm 1.9 \mathrm{~mL} / \mathrm{kg} / \mathrm{min}$, respectively. Mean \pm SD time between the CT-scan and CPET was $15.2 \pm 15.3$ days. CPET results are shown in Table 2 .

Mean \pm SD skeletal muscle mass index was $50.9 \pm 10.6 \mathrm{~cm}^{2} / \mathrm{m}^{2}$ in males (range 31.1 to 91.5 ) and $36.6 \pm 8.1 \mathrm{~cm}^{2} / \mathrm{m}^{2}$ in women (range 20.4 to 66.7 ). CT-scan measurements are depicted in Table 2.

\section{Association between preoperative body composition parameters derived from the abdominal computed tomography-scan and preoperative cardiopulmonary exercise testing parameters}

In the univariable analysis (Table 3$)$, a Pearson correlation coefficient of $0.55(P<0.001)$ was found between the absolute AT and skeletal muscle mass index. Between the relative AT and skeletal muscle mass index, a correlation coefficient of $0.16(P=0.156)$ was observed. A Pearson correlation coefficient of $0.28(P=0.014)$ was found between the relative AT and SM-RA.

Variables with a $P<0.10$ in the univariable analysis (age, body mass, body height, ASA, sex, skeletal muscle mass index, and VAT mass) were included in a multivariable linear regression analysis to predict the absolute AT. BMI was also associated with absolute AT $(P<0.10)$ but was not included in the multivariable analysis because of multicollinearity between $\mathrm{BMI}$, body mass, and body height. In the final multivariable model ( $\left.\mathrm{R}^{2} 51.1 \%\right)$, a lower age, a higher body mass, a lower ASA score, and a higher skeletal muscle mass index were associated with a higher absolute AT (Table 4). In a formula, absolute AT (mL/ $\min )=848.6-(4.99 \times$ age in years $)+(4.18 \times$ body mass in $\mathrm{kg})-(124.4 \times \mathrm{ASA}$ score $)+(4.65 \times$ skeletal muscle mass index in $\left.\mathrm{cm}^{2} / \mathrm{m}^{2}\right)$. For an ASA score 1 or 2 , a 1 must be used, whereas for an ASA score 3 or 4 a 2 should be used in the equation.

Moreover, variables with a $P<0.10$ in the univariable analysis (BMI, ASA, VSAQ score, SM-RA, and VAT mass) were included in the multivariable linear regression analysis to predict the relative AT. Body mass was also associated with relative AT $(P<0.10)$ but was not included in the multivariable analysis because of multicollinearity between body mass and BMI. 
Table 1 Patient characteristics.

\begin{tabular}{|c|c|}
\hline Parameter & Total $(n=78)$ \\
\hline Age (years) & $74.5 \pm 6.4$ \\
\hline Sex (males) & $45(57.7)$ \\
\hline Body height $(\mathrm{cm})$ & $169.9 \pm 9.3$ \\
\hline Males & $175.1 \pm 7.1$ \\
\hline Females & $163.0 \pm 7.2$ \\
\hline Body mass (kg) & $84.5 \pm 14.3$ \\
\hline Males & $89.0 \pm 13.7$ \\
\hline Females & $78.5 \pm 12.9$ \\
\hline Body mass index $\left(\mathrm{kg} / \mathrm{m}^{2}\right)$ & $29.2 \pm 3.8$ \\
\hline Males & $29.0 \pm 3.8$ \\
\hline Females & $29.5 \pm 3.9$ \\
\hline Smoking & $11(15.7)$ \\
\hline VSAQ score $(\mathrm{METS})^{\mathrm{b}}$ & $5 \pm 1$ \\
\hline \multicolumn{2}{|l|}{ Charlson comorbidity index } \\
\hline 0 & $23(29.5)$ \\
\hline 1 & $27(34.6)$ \\
\hline$\geq 2$ & $28(35.9)$ \\
\hline \multicolumn{2}{|l|}{ ASA score } \\
\hline I and II & $61(78.2)$ \\
\hline III and IV & $17(21.8)$ \\
\hline \multicolumn{2}{|l|}{ Tumor localization } \\
\hline Ascending colon & $29(37.2)$ \\
\hline Transverse colon & $7(9.0)$ \\
\hline Descending colon & $5(6.4)$ \\
\hline Sigmoid & $23(29.5)$ \\
\hline Rectum ${ }^{c}$ & $11(14.1)$ \\
\hline Other $^{d}$ & $3(3.8)$ \\
\hline \multicolumn{2}{|l|}{ Clinical metastasis category } \\
\hline cM0 & $67(85.9)$ \\
\hline cM1 & $5(6.4)$ \\
\hline Not applicable & $6(7.7)$ \\
\hline
\end{tabular}

Values are presented as mean \pm SD or as $\mathrm{n}(\%)$.

a: 8 missing values.

b: 13 missing values.

c: 4 patients with a rectal tumor received neoadjuvant chemoradiation, 1 patient received neoadjuvant radiotherapy.

d: 2 patients had a tumor in both the ascending and transverse colon, 1 patient had metachronous colorectal liver metastasis.

e: Includes dysplasia $(n=5)$ and metachronous colorectal liver metastasis $(n=1)$.

Abbreviations: ASA, American Society of Anesthesiologists; MET, metabolic equivalent of task; VSAQ, veteransspecific activity questionnaire. 
Table 2 Preoperative body composition parameters derived from the abdominal CT-scan and preoperative CPET parameters.

\begin{tabular}{|c|c|c|c|c|}
\hline Parameter & Total $(n=78)$ & Males $(n=45)$ & Females $(n=33)$ & $P$-value \\
\hline \multicolumn{5}{|l|}{ CT-scan parameters } \\
\hline $\begin{array}{l}\text { Skeletal muscle mass index }\left(\mathrm{cm}^{2} /\right. \\
\left.\mathrm{m}^{2}\right)\end{array}$ & $44.9 \pm 11.9$ & $50.9 \pm 10.6$ & $36.6 \pm 8.1$ & $<0.001$ \\
\hline SM-RA (HU) & $29.1 \pm 7.6$ & $30.3 \pm 7.8$ & $27.5 \pm 7.2$ & 0.110 \\
\hline VAT mass $\left(\mathrm{cm}^{2} / \mathrm{m}^{2}\right)$ & $77.8 \pm 38.2$ & $86.3 \pm 37.9$ & $66.2 \pm 36.1$ & 0.021 \\
\hline SAT mass $\left(\mathrm{cm}^{2} / \mathrm{m}^{2}\right)$ & $80.0 \pm 30.4$ & $65.2 \pm 26.5$ & $100.1 \pm 22.9$ & $<0.001$ \\
\hline \multicolumn{5}{|l|}{ CPET parameters } \\
\hline $\mathrm{HR}_{\text {peak }}(\text { beats } / \mathrm{min})^{\mathrm{a}}$ & $129 \pm 19$ & $128 \pm 19$ & $130 \pm 19$ & 0.751 \\
\hline Without beta blocker ${ }^{b}$ & $135 \pm 17$ & $137 \pm 15$ & $133 \pm 20$ & 0.429 \\
\hline With beta blocker ${ }^{b}$ & $120 \pm 18$ & $119 \pm 19$ & $122 \pm 18$ & 0.728 \\
\hline $\mathrm{RER}_{\text {peak }}$ & $1.14 \pm 0.11$ & $1.16 \pm 0.10$ & $1.12 \pm 0.11$ & 0.059 \\
\hline$W R_{\text {peak }}(W)$ & $98 \pm 32$ & $110 \pm 32$ & $83 \pm 25$ & $<0.001$ \\
\hline$W_{R_{\text {peak }}}(\mathrm{W} / \mathrm{kg})$ & $1.2 \pm 0.3$ & $1.2 \pm 0.3$ & $1.1 \pm 0.3$ & 0.030 \\
\hline $\mathrm{VO}_{2 \text { peak }}(\mathrm{mL} / \mathrm{min})$ & $1312 \pm 351$ & $1413 \pm 348$ & $1173 \pm 309$ & 0.002 \\
\hline $\mathrm{VO}_{2 \text { peak }}(\mathrm{mL} / \mathrm{kg} / \mathrm{min})$ & $15.6 \pm 3.7$ & $16.0 \pm 3.8$ & $15.1 \pm 3.5$ & 0.262 \\
\hline AT $(\mathrm{mL} / \mathrm{min})^{c}$ & $889 \pm 181$ & $937 \pm 175$ & $824 \pm 171$ & 0.006 \\
\hline AT $(\mathrm{mL} / \mathrm{kg} / \mathrm{min})^{\mathrm{c}}$ & $10.6 \pm 1.9$ & $10.6 \pm 1.9$ & $10.5 \pm 1.7$ & 0.823 \\
\hline $\mathrm{O}_{2}-$ pulse $_{\text {peak }}(\mathrm{mL} / \text { beat })^{\mathrm{a}}$ & $10.3 \pm 2.6$ & $11.2 \pm 2.7$ & $9.0 \pm 2.0$ & $<0.001$ \\
\hline $\mathrm{O}_{2}-$ pulse $_{\text {peak }}(\mathrm{mL} / \mathrm{kg} / \text { beat } \times 100)^{\mathrm{a}, \mathrm{d}}$ & $12.3 \pm 2.3$ & $12.8 \pm 2.6$ & $11.7 \pm 1.9$ & 0.056 \\
\hline VE/VCO ${ }_{2}$-slope $e^{e}$ & $33.2 \pm 6.6$ & $33.8 \pm 7.8$ & $32.4 \pm 4.6$ & 0.375 \\
\hline $\mathrm{VE}_{\text {peak }}(\mathrm{L} / \mathrm{min})$ & $56.6 \pm 17.0$ & $62.5 \pm 16.7$ & $48.7 \pm 14.1$ & $<0.001$ \\
\hline $\mathrm{VE}_{\text {peak }}(\mathrm{L} / \mathrm{kg} / \mathrm{min})$ & $0.7 \pm 0.2$ & $0.7 \pm 0.2$ & $0.6 \pm 0.2$ & 0.094 \\
\hline OUES & $1576 \pm 444$ & $1695 \pm 428$ & $1413 \pm 418$ & 0.005 \\
\hline OUES/kg & $18.7 \pm 4.5$ & $19.2 \pm 4.7$ & $18.0 \pm 4.2$ & 0.248 \\
\hline
\end{tabular}

Values are presented as mean \pm SD.

${ }^{a}$ : Heart rate was invalid in 8 patients $(10.3 \%, 6$ males and 2 females), so in this case $n=70$.

b: A beta-blocker was used by 26 patients (17 males and 9 females), 43 patients did not use a beta blocker, and in 1 patient beta blocker use was unknown.

c: The AT was not determinable in two patients (2.6\%, 1 male and 1 female), so in this case $n=76$.

d: $\mathrm{O}_{2}$-pulse values normalized for body mass are multiplied by 100 to increase readability.

e: The VE/VCO -slope was calculated using data up to the respiratory compensation point.

f: Independent samples t-tests.

Abbreviations: AT, anaerobic threshold; CPET, cardiopulmonary exercise testing; CT, computed tomography; $\mathrm{HR}_{\text {peak }}$, heart rate at peak exercise; $\mathrm{HU}$, Hounsfield Units; $\mathrm{O}_{2}$-pulse peak $_{\text {, }}$ oxygen pulse at peak exercise; OUES, oxygen uptake efficiency slope; $\mathrm{RER}_{\text {peak }}$, respiratory exchange ratio at peak exercise; SAT, subcutaneous adipose tissue; SD, standard deviation; SM-RA, skeletal muscle radiation attenuation; VAT, visceral adipose tissue; $\mathrm{VE}_{\text {peak }}$, minute ventilation at peak exercise; $\mathrm{VE} / \mathrm{VCO}_{2}$-slope, minute ventilation to carbon dioxide production relationship; $\mathrm{VO}_{2 \text { peak }}$, oxygen uptake at peak exercise; $\mathrm{WR}_{\text {peak }}$, work rate at peak exercise. 
Table 3 Correlation coefficients between preoperative body composition parameters derived from the abdominal CT-scan and preoperative CPET parameters.

\begin{tabular}{|c|c|c|c|c|}
\hline Parameter & $\begin{array}{c}\text { Skeletal muscle } \\
\text { mass index } \\
\left(\mathrm{cm}^{2} / \mathbf{m}^{2}\right)\end{array}$ & SM-RA (HU) & VAT mass $\left(\mathrm{cm}^{2} / \mathrm{m}^{2}\right)$ & $\begin{array}{l}\text { SAT mass } \\
\left(\mathrm{cm}^{2} / \mathbf{m}^{2}\right)\end{array}$ \\
\hline AT $(\mathrm{mL} / \mathrm{min})^{\mathrm{a}}$ & $0.55(P<0.001)$ & $0.08(P=0.472)$ & $0.22(P=0.063)$ & $0.03(P=0.783)$ \\
\hline AT $(\mathrm{mL} / \mathrm{kg} / \mathrm{min})^{\mathrm{a}}$ & $0.16(P=0.156)$ & $0.28(P=0.014)$ & $-0.24(P=0.040)$ & $-0.16(P=0.177)$ \\
\hline $\mathrm{VO}_{2 \text { peak }}(\mathrm{mL} / \mathrm{min})$ & $0.51(P<0.001)$ & $0.10(P=0.369)$ & $0.18(P=0.122)$ & $-0.09(P=0.427)$ \\
\hline $\mathrm{VO}_{2 \text { peak }}(\mathrm{mL} / \mathrm{kg} / \mathrm{min})$ & $0.22(P=0.058)$ & $0.26(P=0.020)$ & $-0.17(P=0.130)$ & $-0.24(P=\mathbf{0 . 0 3 4})$ \\
\hline VE/ $/ \mathrm{VCO}_{2}$-slope ${ }^{\mathrm{b}}$ & $-0.12(P=0.281)$ & $-0.17(P=0.127)$ & $-0.02(P=0.889)$ & $-0.10(P=0.390)$ \\
\hline OUES & $0.40(\boldsymbol{P}<0.001)$ & $<-0.01(P=0.991)$ & $0.23(\boldsymbol{P}=\mathbf{0 . 0 4 5})$ & $<-0.01(P=0.979)$ \\
\hline OUES/kg & $0.13(P=0.246)$ & $0.15(P=0.202)$ & $-0.12(P=0.287)$ & $-0.18(P=0.120)$ \\
\hline
\end{tabular}

In the final multivariable model ( $\mathrm{R}^{2} 28.6 \%$ ), a higher $\mathrm{BMI}$, a higher ASA score, and a lower SM-RA were associated with a lower relative AT (Table 4). In a formula, relative AT ( $\mathrm{mL} / \mathrm{kg} /$ $\min )=15.1-\left(0.13 \times \mathrm{BMI}\right.$ in $\left.\mathrm{kg} / \mathrm{m}^{2}\right)-(1.80 \times \mathrm{ASA}$ score $)+(0.05 \times \mathrm{SM}-\mathrm{RA})$. For an ASA score 1 or 2, a 1 must be used, whereas for an ASA score 3 or 4 a 2 should be used in the formula. The multivariable linear regression analyses to predict the absolute and relative $\mathrm{VO}_{2 \text { peak }}$ can be found in Table 4.

\section{Prediction of a preoperative relative anaerobic threshold $<$ II.I mL/kg/min}

A multivariable logistic regression analysis was performed to investigate if a preoperative relative $A T \leq 11.1 \mathrm{~mL} / \mathrm{kg} / \mathrm{min}$ can be predicted from body composition variables derived from the abdominal CT-scan and other patient characteristics. In the univariable analysis, age, body mass, BMI, VAT mass, ASA score, VSAQ score, and Charlson score were associated with a relative $A T \leq 11.1 \mathrm{~mL} / \mathrm{kg} / \mathrm{min}$ (with a $P<0.10$ ) and were included in a forward stepwise multivariable analysis. A higher ASA score (odds ratio [OR] 6.95, 95\% confidence interval $(\mathrm{Cl}) 0.81$ to $59.3, P=0.076$ ) and a higher VAT mass (OR $1.01,95 \% \mathrm{Cl} 1.00$ to 1.03 , $P=0.090$ ) were associated with an increased risk of a relative $A T \leq 11.1 \mathrm{~mL} / \mathrm{kg} / \mathrm{min}$. Another logistic regression model was made (method: enter) with ASA and VAT mass, to include all patients (as, though $\leq 7 \mathrm{METs}$, the exact VSAQ score of thirteen patients were missing). In this final model, a higher ASA score (OR 5.64, 95\% Cl 1.15 to $27.7, P=0.033$ ) and a higher VAT mass (OR $1.02,95 \% \mathrm{Cl} 1.00$ to $1.03, P=0.036$ ) were associated with an increased risk of a relative $A T \leq 11.1 \mathrm{~mL} / \mathrm{kg} / \mathrm{min}$. Patients with ASA score of 3 or 4 were almost six times more likely to have a relative $A T \leq 11.1 \mathrm{~mL} / \mathrm{kg} / \mathrm{min}$. 
Table 4 Multivariable linear regression analysis to predict the preoperative absolute and relative AT and absolute and relative $\mathrm{VO}_{2 \text { peak }}$.

\begin{tabular}{|c|c|c|c|c|}
\hline Predicted CPET variable & Parameter & B & $95 \% \mathrm{Cl}$ & $P$-value \\
\hline \multirow[t]{4}{*}{ Absolute AT (mL/min) } & Age (years) & -5.00 & -9.80 to -0.19 & 0.042 \\
\hline & Body mass (kg) & 4.18 & 1.69 to 6.66 & 0.001 \\
\hline & ASA score & -124 & -199 to -49.8 & 0.001 \\
\hline & $\begin{array}{l}\text { Skeletal muscle mass index } \\
\left(\mathrm{cm}^{2} / \mathrm{m}^{2}\right)\end{array}$ & 4.65 & 1.69 to 7.62 & 0.003 \\
\hline \multirow[t]{3}{*}{ Relative AT (mL/kg/min) } & Body mass index $\left(\mathrm{kg} / \mathrm{m}^{2}\right)$ & -0.13 & -0.23 to -0.03 & 0.014 \\
\hline & ASA score & -1.80 & -2.70 to -0.90 & $<0.001$ \\
\hline & SM-RA (HU) & 0.05 & -0.004 to 0.10 & 0.071 \\
\hline \multirow[t]{4}{*}{ Absolute $\mathrm{VO}_{\text {2peak }}{ }^{\mathrm{a}}(\mathrm{mL} / \mathrm{min})$} & Age (years) & -12.0 & -21.3 to -2.63 & 0.013 \\
\hline & Body height $(\mathrm{cm})$ & 12.5 & 5.34 to 19.7 & 0.001 \\
\hline & ASA score & -270 & -413 to -128 & $<0.001$ \\
\hline & $\begin{array}{l}\text { Skeletal muscle mass index } \\
\left(\mathrm{cm}^{2} / \mathrm{m}^{2}\right)\end{array}$ & 8.22 & 2.69 to 13.8 & 0.004 \\
\hline \multirow{5}{*}{$\begin{array}{l}\text { Relative } \mathrm{VO}_{2 \text { peak }} \mathrm{b}^{\mathrm{b}}(\mathrm{mL} / \mathrm{kg} / \\
\text { min) }\end{array}$} & Age (years) & -0.14 & -0.24 to -0.04 & 0.008 \\
\hline & Body mass index $\left(\mathrm{kg} / \mathrm{m}^{2}\right)$ & -0.42 & -0.59 to -0.25 & $<0.001$ \\
\hline & ASA score & -2.40 & -4.11 to -0.69 & 0.007 \\
\hline & Charlson comorbidity index & -1.12 & -1.98 to -0.26 & 0.012 \\
\hline & $\begin{array}{l}\text { Skeletal muscle mass index } \\
\left(\mathrm{cm}^{2} / \mathrm{m}^{2}\right)\end{array}$ & 0.09 & 0.03 to 0.15 & 0.003 \\
\hline
\end{tabular}

a: In a formula, absolute $\mathrm{VO}_{2 \text { peak }}(\mathrm{mL} / \mathrm{min})=34.9-(12.0 \times$ age in years $)+(12.5 \times$ body height in $\mathrm{cm})-(270 \times$ ASA score $)+\left(8.22 \times\right.$ skeletal muscle mass index in $\left.\mathrm{cm}^{2} / \mathrm{m}^{2}\right)$. For an ASA score 1 or 2 , a 1 must be used, whereas for an ASA score 3 or 4 a 2 should be used in the equation.

b: In a formula, relative $\mathrm{VO}_{2 \text { peak }}(\mathrm{mL} / \mathrm{kg} / \mathrm{min})=38.4-(0.14 \times$ age in years $)-\left(0.42 \times \mathrm{BMI}\right.$ in $\left.\mathrm{kg} / \mathrm{m}^{2}\right)-(2.40 \times \mathrm{ASA}$ score) $-(1.12 \times$ Charlson score $)+\left(0.09 \times\right.$ skeletal muscle mass index in $\left.\mathrm{cm}^{2} / \mathrm{m}^{2}\right)$. For an ASA score 1 or 2 , a 1 must be used, whereas for an ASA score 3 or 4 a 2 should be used in the equation. For a Charlson score 0 , a 0 should be used, for a Charlson score 1, a 1 must be used, and for a Charlson score 2+, a 2 should be used in the equation. Abbreviations: ASA, American Society of Anesthesiologists; AT, anaerobic threshold; $\mathrm{Cl}$, confidence interval; $\mathrm{HU}$, Hounsfield Units; SM-RA, skeletal muscle radiation attenuation; $\mathrm{VO}_{2 \text { peak }}$, oxygen uptake at peak exercise.

ROC analysis for predicting patients with a relative AT $\leq 11.1 \mathrm{~mL} / \mathrm{kg} / \mathrm{min}$ from ASA score and VAT mass gave an AUC of $0.71(95 \% \mathrm{Cl} 0.60$ to $0.83, P=0.002)$ (Figure 1$)$. Patients with a relative $A T \leq 11.1 \mathrm{~mL} / \mathrm{kg} / \mathrm{min}$ can be predicted with the formula: $1 / 1+\mathrm{e}(-(-0.74+(0.02 \times$ VAT mass $)+(1.73 \times$ ASA $))$. For an ASA score 1 or 2 , a 0 must be used, whereas for an ASA score 3 or 4 a 1 should be used in the equation. When choosing a cut-off point of 0.55 , sensitivity was $82.7 \%$, and specificity was $46.2 \%$, while the positive predictive value was $75.4 \%$ and the negative predictive value was $57.1 \%$. 


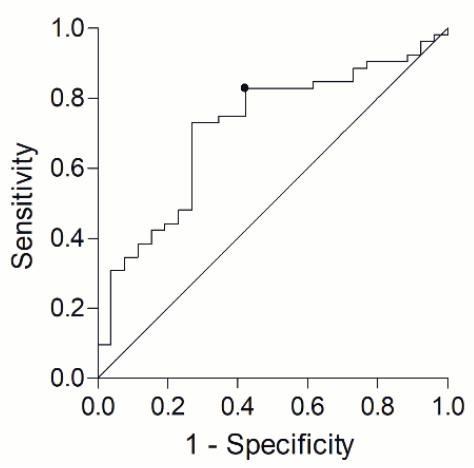

Figure $1 \mathrm{ROC}$ analysis for predicting patients with a relative $\mathrm{AT} \leq 11.1 \mathrm{~mL} / \mathrm{kg} / \mathrm{min}$ from ASA score and visceral adipose tissue (AUC $0.71 ; 95 \% \mathrm{Cl} 0.60$ to $0.83 ; P=0.002$ ).

Abbreviations: ASA, American Society of Anesthesiologists; AT, anaerobic threshold; AUC, area under the curve; $\mathrm{Cl}$, confidence interval; ROC, receiver operator characteristic.

\section{Discussion}

This study aimed to investigate the association between body composition variables derived from the preoperative abdominal CT-scan and preoperative CPET variables of aerobic fitness in patients scheduled for colorectal surgery, to evaluate whether the preoperative CT-scan can (assist to pre)select unfit patients. Results demonstrated that body composition variables were significantly associated with preoperative aerobic fitness, expressed as the absolute and relative $\mathrm{AT}$, absolute and relative $\mathrm{VO}_{2 \text { peak }}$, and OUES. In the multivariable regression model to predict the preoperative absolute AT, it was found that the absolute AT ( $\mathrm{R}^{2}$ 51.1\%) was lower in patients with a lower skeletal muscle mass index, together with a higher age, a lower body mass, and a higher ASA score. Variation in relative AT values ( $R^{2} 28.6 \%$ ) could be less explained by body composition variables and other patient-related variables.

Body composition variables such as skeletal muscle mass correlate better with absolute measures of aerobic fitness $\left(\mathrm{AT}, \mathrm{VO}_{2 \text { peak }}\right.$, and OUES) than with relative variables (here normalized for body mass) of aerobic fitness. This can be explained by the fact that skeletal muscle mass represents an absolute measure of the body's skeletal muscle mass, and a higher absolute skeletal muscle mass generally results in greater exercise-induced peripheral oxygen extraction and utilization by the exercising muscles, which is an important determinant for absolute aerobic fitness. Aerobic fitness refers to the maximal capacity of the pulmonary and cardiovascular system to take in and transport oxygen to the exercising muscles, and of those exercising muscles to extract and utilize oxygen from the blood for aerobic respiration. ${ }^{22}$ Thus, aerobic fitness not merely depends on skeletal muscle mass and SM-RA, which might explain the weak-to-moderate correlation 
coefficients found in the current study. Findings of the current study are consistent with the literature in which aerobic fitness was significantly reduced in patients with low skeletal muscle mass index. ${ }^{23-25}$ However, limited research is available that describes the association between aerobic fitness objectively measured with CPET and body composition variables derived from the abdominal CT-scan. In a recent study, West and others ${ }^{16}$ assessed the association of CT-scan derived body composition with selected CPET variables in patients scheduled for hepatopancreatobiliary and pancreatic surgery. They demonstrated that patients with lower SM-RA values had a statistically significantly lower relative AT $(r 0.44, P<0.001)$ and relative $\mathrm{VO}_{2 \text { peak }}(r 0.57, P<0.001)$. In the current study, it was also found that SM-RA was significantly correlated with relative AT and relative $\mathrm{VO}_{2 \text { peak }}$ in the univariate analysis (Table 3); however, SM-RA values were not statistically significantly associated with relative AT and relative $\mathrm{VO}_{2 \text { peak }}$ in the multivariable model (Table 4). Concerning skeletal muscle mass index, West and others ${ }^{16}$ reported a weak association ( $\mathrm{r}$ 0.24, $P=0.010$ ) with relative $\mathrm{VO}_{2 \text { peak }}$. In agreement with the current study results, no significant correlation coefficient was found between skeletal muscle mass index and relative AT.

A previous study has shown that patients undergoing major elective colorectal surgery with an AT $\leq 11.1 \mathrm{~mL} / \mathrm{kg} / \mathrm{min}$ have an increased risk for postoperative complications (OR $7.56,95 \% \mathrm{Cl} 4.44$ to $12.86, P<0.001) .{ }^{8}$ Therefore, this study investigated whether a patient with a relative AT $\leq 11.1 \mathrm{~mL} / \mathrm{kg} / \mathrm{min}$ could be predicted from body composition variables derived from the preoperative abdominal CT-scan combined with other patient characteristics. A higher ASA score and a higher VAT mass were associated with an increased risk of a relative AT $\leq 11.1 \mathrm{~mL} / \mathrm{kg} / \mathrm{min}$. However, with an AUC of 0.71 , the combination of ASA score and VAT mass had only a moderate ability to discriminate between patients with and without a relative AT $\leq 11.1 \mathrm{~mL} / \mathrm{kg} / \mathrm{min}$. Nevertheless, this finding suggests that preoperatively assessing body composition from the routinely performed preoperative CT-scan, combined with other patient-related variables, might be useful to enable a preselection of potentially unfit patients, without the need for using additional questionnaires or tests. These potentially unfit (high-risk) patients should subsequently perform a preoperative CPET to determine the need for a preoperative preventive intervention (e.g., multimodal prehabilitation to improve preoperative aerobic capacity and muscle mass). This preselection might reduce the number of preoperative CPET procedures, thereby saving time and resources.

Preoperative risk assessment is important, as it is the less physically fit patient that will benefit the most from prehabilitation. ${ }^{26,27}$ Despite mounting evidence that prehabilitation has the potential to improve preoperative physical fitness and postoperative outcomes, ${ }^{28,29}$ there remains work to be done in order to develop a cost-effectiveness tool that gives clinicians and policy makers insight in the value of preoperative risk assessment followed 
by preventive interventions in the right patients. As our results suggest, body composition variables derived from the routinely performed abdominal CT-scan, together with other patient characteristics, provides at best limited information on a patient's aerobic fitness. Therefore, the relatively complex and expensive CPET cannot be fully replaced by the preoperative abdominal CT scan. The extent to which other, less sophisticated, tests like the steep ramp test, timed up-and-go test, six-minute walk test, and short physical performance battery could refer to preoperative aerobic fitness remains to be evaluated.

The explorative nature of the study, the limited number of patients, the absence of a prospective sample size calculation are limitations of the present study. Additionally, the fact that only patients with a VSAQ score $\leq 7$ METs were referred for CPET might have biased the results, as having all patients perform a CPET prior to colorectal surgery probably would lead to greater accuracy in determining the association between preoperative CT scan-derived body composition variables and preoperative aerobic fitness. These aspects affect statistical analysis and generalizability. Moreover, the studied population is limited to patients undergoing colorectal surgery, who do not necessarily represent the general (surgical) population.

\section{Conclusion}

Body composition variables derived from the preoperative CT-scan are moderately associated with aerobic fitness as determined from the preoperative CPET. A higher ASA score and a higher VAT mass were associated with an increased risk of a relative AT $\leq 11.1$ $\mathrm{mL} / \mathrm{kg} / \mathrm{min}$ as a cut-off to classify patients scheduled for colorectal surgery as having an increased risk for postoperative morbidity. It seems the CT-scan cannot replace the CPET for preoperative risk assessment on aerobic fitness; however, it may contribute to the (pre)selection of unfit patients. 


\section{References}

1. Bray F, Ferlay J, Soerjomataram I, Siegel RL, Torre LA, Jemal A. Global cancer statistics 2018: GLOBOCAN estimates of incidence and mortality worldwide for 36 cancers in 185 countries. CA Cancer J Clin 2018; 68(6): 394-424.

2. Dutch institute for clinical auditing (DICA) Leiden. DICA jaarrapportage 2018: Dutch ColoRectal Audit (DCRA). Available at: https://dica.nl/jaarrapportage-2018/dcra. Last accessed June 10, 2020.

3. Govaert JA, Fiocco M, van Dijk WA, et al. Costs of complications after colorectal cancer surgery in the Netherlands: Building the business case for hospitals. Eur J Surg Oncol 2015; 41(8): 1059-67.

4. Hulzebos EH, van Meeteren NL. Making the elderly fit for surgery. Br J Surg 2016; 103(4): 463.

5. Levett DZH, Jack S, Swart M, et al. Perioperative cardiopulmonary exercise testing (CPET): consensus clinical guidelines on indications, organization, conduct, and physiological interpretation. Br J Anaesth 2018; 120(3): 484-500.

6. West MA, Lythgoe D, Barben CP, Noble L, Kemp GJ, Jack S, Grocott MP. Cardiopulmonary exercise variables are associated with postoperative morbidity after major colonic surgery: a prospective blinded observational study. Br J Anaesth 2014; 112(4): 665-71.

7. West MA, Parry MG, Lythgoe D, Barben CP, Kemp GJ, Grocott MP, Jack S. Cardiopulmonary exercise testing for the prediction of morbidity risk after rectal cancer surgery. Br J Surg 2014; 101(9): 1166-72.

8. West MA, Asher R, Browning M, et al. Validation of preoperative cardiopulmonary exercise testing-derived variables to predict in-hospital morbidity after major colorectal surgery. $\mathrm{Br} \mathrm{J}$ Surg 2016; 103(6): 744-52.

9. Moran J, Wilson F, Guinan E, McCormick P, Hussey J, Moriarty J. Role of cardiopulmonary exercise testing as a risk-assessment method in patients undergoing intra-abdominal surgery: a systematic review. Br J Anaesth 2016; 116(2): 177-91.

10. Jones K, Gordon-Weeks A, Coleman C, Silva M. Radiologically Determined Sarcopenia Predicts Morbidity and Mortality Following Abdominal Surgery: A Systematic Review and Meta-Analysis. World J Surg 2017; 41(9): 2266-79.

11. Berkel AEM, Klaase JM, de Graaff F, Brusse-Keizer MGJ, Bongers BC, van Meeteren NLU. Patient's Skeletal Muscle Radiation Attenuation and Sarcopenic Obesity are Associated with Postoperative Morbidity after Neoadjuvant Chemoradiation and Resection for Rectal Cancer. Dig Surg 2019; 36(5): 376-83.

12. Sabel MS, Terjimanian M, Conlon AS, et al. Analytic morphometric assessment of patients undergoing colectomy for colon cancer. J Surg Oncol 2013; 108(3): 169-75.

13. Schroder FF, de Graaff F, Bouman DE, Brusse-Keizer M, Slump KH, Klaase JM. The Preoperative CT-Scan Can Help to Predict Postoperative Complications after Pancreatoduodenectomy. Biomed Res Int 2015; 2015: 824525. 
14. Kuritzkes BA, Pappou EP, Kiran RP, et al. Visceral fat area, not body mass index, predicts postoperative 30-day morbidity in patients undergoing colon resection for cancer. Int $J$ Colorectal Dis 2018; 33(8): 1019-28.

15. Boo SH, Joo MC, Lee JM, Kim SC, Yu YM, Kim MS. Association between skeletal muscle mass and cardiorespiratory fitness in community-dwelling elderly men. Aging Clin Exp Res 2019; 31(1): 49-57.

16. West MA, van Dijk DPJ, Gleadowe F, et al. Myosteatosis is associated with poor physical fitness in patients undergoing hepatopancreatobiliary surgery. J Cachexia Sarcopenia Muscle 2019; 10(4): 860-71.

17. Snowden CP, Prentis JM, Anderson HL, Roberts DR, Randles D, Renton M, Manas DM. Submaximal cardiopulmonary exercise testing predicts complications and hospital length of stay in patients undergoing major elective surgery. Ann Surg 2010; 251(3): 535-41.

18. Taaffe DR, Henwood TR, Nalls MA, Walker DG, Lang TF, Harris TB. Alterations in muscle attenuation following detraining and retraining in resistance-trained older adults. Gerontology 2009; 55(2): 217-23.

19. American Thoracic Society, American College of Chest Physicians. ATS/ACCP Statement on cardiopulmonary exercise testing. Am J Respir Crit Care Med 2003; 167(2): 211-77.

20. Beaver WL, Wasserman K, Whipp BJ. A new method for detecting anaerobic threshold by gas exchange. J Appl Physiol (1985) 1986; 60(6): 2020-7.

21. Bongers BC, Berkel AE, Klaase JM, van Meeteren NL. An evaluation of the validity of the preoperative oxygen uptake efficiency slope as an indicator of cardiorespiratory fitness in elderly patients scheduled for major colorectal surgery. Anaesthesia 2017; 72(10): 1206-16.

22. Bongers BC, van Brussel M, Hulzebos HJ, Takken T. Pediatric norms for cardiopulmonary exercise testing: in relation to sex and age (second edition). 's Hertogenbosch, the Netherlands: Uitgeverij BOXpress, 2014.

23. de Oliveira RJ, Bottaro M, Motta AM, et al. Association between sarcopenia-related phenotypes and aerobic capacity indexes of older women. J Sports Sci Med 2009; 8(3): 337-43.

24. Sanada K, Kuchiki T, Miyachi M, McGrath K, Higuchi M, Ebashi H. Effects of age on ventilatory threshold and peak oxygen uptake normalised for regional skeletal muscle mass in Japanese men and women aged 20-80 years. Eur J Appl Physiol 2007; 99(5): 475-83.

25. Kim TN, Park MS, Kim YJ, et al. Association of low muscle mass and combined low muscle mass and visceral obesity with low cardiorespiratory fitness. PLoS One 2014; 9(6): e100118.

26. Thomas G, Tahir MR, Bongers BC, Kallen VL, Slooter GD, van Meeteren NL. Prehabilitation before major intra-abdominal cancer surgery: A systematic review of randomised controlled trials. Eur J Anaesthesiol 2019; 36(12): 933-45.

27. Bongers BC, Punt IM, van Meeteren NL. On "Prehabilitation: The emperor's new clothes or a new arena for physical therapists?" Lundberg M, Archer KR, Larsson C, Rydwik E. Phys Ther. 2018;12:127-130. Phys Ther 2019; 99(7): 953-4. 
28. Barberan-Garcia A, Ubre M, Roca J, et al. Personalized Prehabilitation in High-risk Patients Undergoing Elective Major Abdominal Surgery: A Randomized Blinded Controlled Trial. Ann Surg 2018; 267(1): 50-6.

29. Berkel AEM, Bongers BC, Kotte $\mathrm{H}$, et al. Effects of Community-based Exercise Prehabilitation for Patients Scheduled for Colorectal Surgery With High Risk for Postoperative Complications: Results of a Randomized Clinical Trial. Ann Surg 2021. Epub ahead of print. 



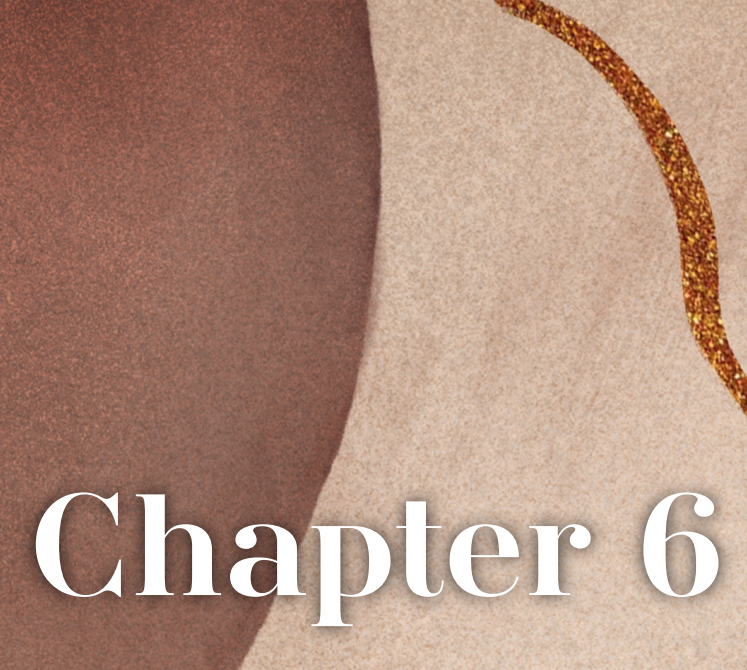

The effects of prehabilitation versus usual care to reduce postoperative complications in high-risk patients with colorectal cancer or dysplasia schedtuled for elective colorectal resection: study protocol of a randomized controlled trial

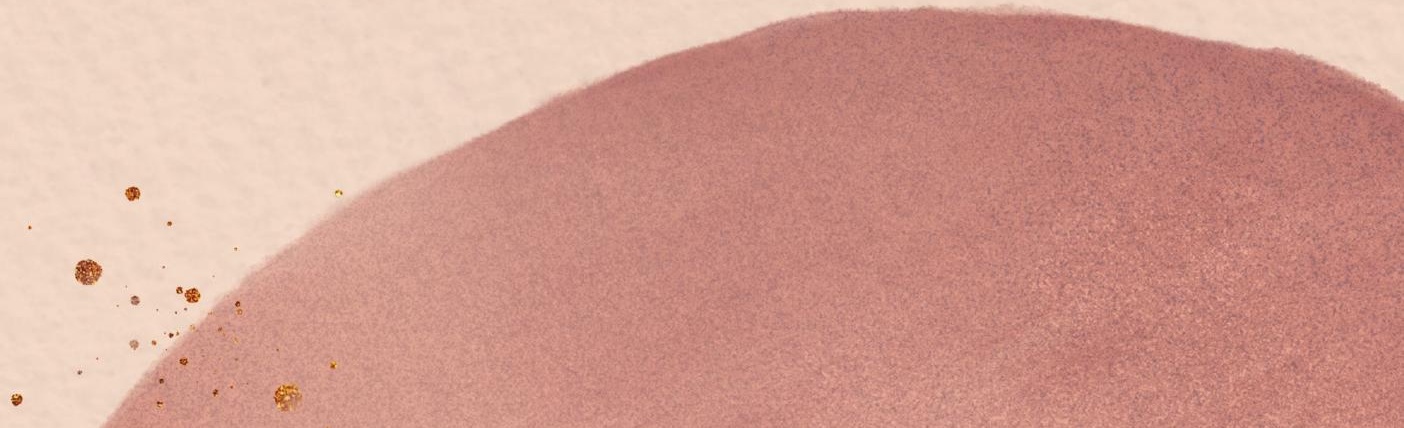

Annefleur E.M. Berkel, Bart C. Bongers, Marie-Janne S. van Kamp, Hayke Kotte, Paul Weltevreden, Frans H.C. de Jongh, Michiel M.M. Eijsvogel, A.N. Machteld Wymenga, Marloes Bigirwamungu-Bargeman, Job van der Palen, Marc J. van Det, Nico L.U. van Meeteren, Joost M. Klaase 


\section{Abstract}

Background: Of all older patients that opt for elective colorectal surgery, approximately one-third has one or more postoperative complications, particularly those patients with low cardiorespiratory fitness (ventilatory anaerobic threshold (VAT) $<11 \mathrm{~mL} / \mathrm{kg} / \mathrm{min}$ ). A physical exercise training program prior to surgery (prehabilitation) can improve their cardiorespiratory fitness. It remains to be seen whether prehabilitation also reduces postoperative complications, as most of the studies so far were rather underpowered, heterogeneous, and biased towards selection of patients with low risk of postoperative complications. The primary objective of this study is to evaluate the effects of a threeweek prehabilitation program on 30-day postoperative complications in patients with a VAT $<11 \mathrm{~mL} / \mathrm{kg} / \mathrm{min}$ planned for elective colorectal resection for colorectal cancer or dysplasia.

Methods: In this multicenter prospective randomized controlled trial, patients $\geq 60$ years with colorectal cancer or dysplasia grade I, II, or III, planned for elective colorectal resection in two hospitals in the Netherlands, will be recruited. Eligible patients must have a score $\leq 7$ metabolic equivalents on the veterans-specific activity questionnaire and should be able to perform a cardiopulmonary exercise test. A total of 86 patients will be randomized (block-stratified randomization) to prehabilitation (intervention group) or usual care. For final inclusion, VAT should be $<11 \mathrm{~mL} / \mathrm{kg} / \mathrm{min}$. Three times a week for three weeks, a 60-minute supervised prehabilitation session will be completed by the 43 patients in the prehabilitation group, consisting of moderate-to-high intensity interval training to improve cardiorespiratory fitness, and resistance training to improve peripheral muscle strength. Additionally, patients perform home exercises twice a week on a moderate intensity level. The 43 patients in the usual care group will receive usual care.

Discussion: Optimizing preoperative physical fitness may decrease the postoperative complication rate, may lead to fewer reoperations, less intense clinical care, a shorter length of stay, a more effective surgical planning (process-optimization), fewer readmissions, less intense rehabilitation, shorter rehabilitation period, earlier resumption of work, enhance patient perceived health-related quality of life, and promote performance in daily life. Cost effectiveness should therefore be expected and evaluated.

Trial registration: Medical Ethics Committee Twente, Enschede, the Netherlands (NL45001.044.13, September 3, 2013); Netherlands Trial Register (NTR; NTR4032, June 14, 2013). 


\section{Introduction}

Worldwide, colorectal cancer is the third most common type of cancer for men and women. ${ }^{1}$ In the Netherlands, about 15.000 people are diagnosed with colorectal cancer each year. ${ }^{2}$ Surgery is the cornerstone of treatment in patients with colorectal cancer. However, the complication rate after elective colorectal resection is approximately onethird, ${ }^{3}$ including for example an anastomotic leakage, ileus, or wound infection. Colorectal cancer primarily occurs in the elderly. The level of psychophysiological reserve capacity (resilience) and comorbidities affect the tolerance to surgery in older patients. ${ }^{4}$ According to the Dutch Surgical Colorectal Audit, approximately 35\% of the patients with colorectal cancer are aged between 60 and 70 years, and $40-50 \%$ of the patients are aged over 70 years. ${ }^{5}$ These older patients more often have a low cardiorespiratory fitness.

In the literature, preoperative cardiorespiratory fitness has consistently been reported to be associated with postoperative outcome in major elective intra-abdominal surgery (e.g., morbidity, mortality, and length of stay). ${ }^{6}$ Cardiorespiratory fitness can be measured objectively using a cardiopulmonary exercise test (CPET) by determining the ventilatory anaerobic threshold (VAT). The VAT is an accurate and repeatable measurement ${ }^{7}$ that can be obtained from the CPET, without a learning effect. ${ }^{8}$ It is a physiological construct that occurs at submaximal exercise intensity. Hence, the VAT is only marginally influenced by the patient's ability and motivation to deliver a maximal effort. Previous studies showed that there is a significant association between a low VAT and postoperative complications in major non-cardiac surgery. ${ }^{8-21}$ Patients with a VAT $<11 \mathrm{~mL} / \mathrm{kg} / \mathrm{min}$ have a higher likelihood for postoperative complications. ${ }^{17,22}$

The literature shows that high-risk patients (those patients with a higher likelihood of postoperative complications), who participated in a physical exercise training program prior to elective surgery (prehabilitation), improved their cardiorespiratory fitness, ${ }^{23-25}$ which has led to the hypothesis that postoperative complications can be reduced by prehabilitation..$^{23,25-27}$ Dunne et al. ${ }^{28}$ designed a four-week high-intensity interval training program based on the work rate at the VAT for patients prior to hepatic resection. Their aim was to assess feasibility and to improve the preoperative VAT by $1.5 \mathrm{~mL} / \mathrm{kg} / \mathrm{min}$ (a $10 \%$ increase). They found their program to be feasible in sedentary healthy volunteers, resulting in a $>10 \%$ improvement in cardiorespiratory fitness (VAT). Using preoperative risk stratification, a 10\% improvement in cardiorespiratory fitness in patients undergoing hepatic resection would shift $30 \%$ of the high-risk patient group to a low-risk patient group. ${ }^{28}$

Prehabilitation programs may have beneficial effects on postoperative outcome; however, the effects on physical functioning, mortality, and length of stay are inconclusive. In a 
systematic review of Moran et al. ${ }^{6}$ that aimed to assess the ability of prehabilitation to influence postoperative outcome after intra-abdominal surgery, it was concluded that prehabilitation appears to be beneficial in decreasing the incidence of postoperative complications. Additionally, a systematic review of Bruns et al. ${ }^{29}$ reported that prehabilitation can improve the physical fitness of older patients undergoing colorectal surgery; however, no significant effect on the reduction of complications or length of stay could be demonstrated. They concluded that previous studies on the effects of prehabilitation in patients undergoing elective colorectal resection provided no clear and complete description of the content and execution of the prehabilitation program, and that in most of these studies no adequate sample size calculation was performed. ${ }^{29} \mathrm{Hijazi}$ et al. ${ }^{30}$ recently performed a systematic review of all comparative studies on prehabilitation versus usual care in patients undergoing abdominal cancer surgery. No significant difference was found in postoperative complications between prehabilitation and usual care groups. They concluded that prehabilitation programs for patients undergoing major abdominal cancer surgery remain heterogeneous in terms of their composition, duration, mode of administration, compliance, and outcome measures used to quantify their impact. Therefore, the findings and recommendations are limited. They recommended for future research to standardize these aspects prior to the evaluation of the effects of prehabilitation programs on a larger scale. ${ }^{30}$ Thus, it remains to be demonstrated whether a prehabilitation program reduces postoperative complications. ${ }^{31}$ Previous studies are biased towards selection of patients with low risk of postoperative complications, and it is unlikely that a prehabilitation program of a few weeks will give a clinically relevant improvement of cardiorespiratory fitness in these low-risk patients.

In summary, current studies addressing the effects of prehabilitation on overall postoperative complications in patients with colorectal cancer are inconclusive, opposing, and of low-to-moderate methodological quality and/or therapeutic validity (concept of Hoogeboom et al. ${ }^{32}$ ). More high-quality studies are needed to validate its use in the preoperative setting. ${ }^{6,33}$ Recently though, it has been demonstrated that personalized prehabilitation reduced the number of patients with postoperative complications by $51 \%$ in high-risk patients undergoing elective major abdominal surgery. ${ }^{24}$ Nevertheless, the selection of high-risk patients in this study of Barberan-Garcia et al. ${ }^{24}$ was not performed by the use of an objective test, such as a preoperative CPET. Based on the previous published work, the current study will be different in several ways: 1) we perform a validated procedure of preoperative risk stratification with each eligible patients, in order to include only patients with a higher risk for postoperative complications based on cardiorespiratory fitness, 2) we preoperatively measure cardiorespiratory fitness objectively by using a CPET to assess the VAT for risk stratification, 3) we design a personalized prehabilitation program based on the results of the CPET, 4) we train at a physical therapy practice close to the patient's home and not at the outpatient clinic of the hospital, as high-risk patients 
and their informal caregivers are less or even unable and/or less motivated to visit the hospital to train three times a week during the preoperative period, 5) we give an exact description of the content and execution of the prehabilitation program, 6) we select a patient group with a homogeneous diagnosis, that is patients with colorectal cancer or dysplasia only, and 7) we performed an adequate sample size calculation based on previous data on the effect of prehabilitation in patients undergoing elective hepatic resection, ${ }^{25,28}$ as well as on the reported complication rates in frail patients undergoing colorectal resection presented by Robinson et al. ${ }^{34}$

\section{Objectives}

The primary objective of this randomized controlled trial is to evaluate the hypothesis that a three-week prehabilitation program (intervention group) will reduce the number of postoperative complications from $50 \%$ to $20 \%$ in patients with a preoperative VAT $<11 \mathrm{~mL} /$ $\mathrm{kg} / \mathrm{min}$ who will undergo elective colorectal resection for colorectal cancer or dysplasia grade I, II, or III, when compared to usual care (control group).

The secondary objectives are 1) to observe whether changes in preoperative cardiorespiratory fitness occur in the intervention group (prehabilitation, single-arm study design), 2) to evaluate the effect of the prehabilitation program on length of stay, 3) to examine whether patients with a preoperative VAT $\geq 11 \mathrm{~mL} / \mathrm{kg} / \mathrm{min}$, and who will therefore not be included in the randomized controlled trial, have fewer postoperative complications than patients in the prehabilitation or usual care group (for the prehabilitation group, we will use the post-prehabilitation (PET data), 4) to investigate the value of a limited geriatric assessment in this patient group to perform preoperative risk stratification, and

5) to evaluate the cost-effectiveness of prehabilitation in high-risk patients by performing a cost-effectiveness analysis.

\section{Methods}

\section{Study design}

This study is a multicenter prospective randomized controlled trial. The trial has started in February 2014 and will run till patient inclusion is completed (probably at the end of 2018) at Medisch Spectrum Twente in Enschede and Ziekenhuisgroep Twente in Almelo, two large community teaching hospitals in the eastern part of the Netherlands. In this manuscript, the latest version of the study protocol (version 9, March 2017) is presented. The study is approved by the Medical Ethics Committee Twente in Enschede, the Netherlands (registration number P13-18), and is registered in the Netherlands Trial Register (NTR4032). Protocol amendments will be agreed and approved by the Medical Ethics Committee Twente. 


\section{Participants}

To be eligible to participate in this study, a patient must meet all of the following inclusion criteria: 1) $\geq 60$ years, 2) colorectal cancer or premalignant colorectal lesions (dysplasia grade I, II, or III) requiring colorectal resection, 3) undergoing elective colorectal resection at Medisch Spectrum Twente, Enschede, or at Ziekenhuisgroep Twente, Almelo, 4) having a life expectancy of more than six months as estimated by the surgeon, 5) has given informed consent to participate in this study, 6) a metabolic equivalents of task (MET) score $\leq 7$ on the veterans-specific activity questionnaire (VSAQ), 7) able to perform a CPET, 8) a VAT $<11 \mathrm{~mL} / \mathrm{kg} / \mathrm{min}$ as measured at the baseline CPET, and 9) willing to perform prehabilitation at a community physical therapy practice in the adherence area of both hospitals. All patients not meeting these criteria will not be considered for inclusion. Participants can leave the study at any time for any reason if they wish to do so, without any consequences. The investigator can decide to withdraw a participant from the study for urgent medical reasons.

\section{Recruitment}

All patients will be identified at the multi-disciplinary oncology meetings and will be evaluated at the outpatient clinic by the surgeon or surgical resident. As most postoperative complications are to be expected in patients with a low cardiorespiratory fitness, ${ }^{6}$ we aim to include only patients who are expected to be unfit in the pre-study assessments. To select patients with a potentially low cardiorespiratory fitness, all patients identified at the multi-disciplinary oncology meeting are asked to fill out the VSAQ. ${ }^{35}$ The VSAQ links physical activities to a particular MET score, based on the Compendium of Physical Activities. ${ }^{36}$ Snowden et al. ${ }^{14}$ found that patients with a VSAQ score > 7 METs are most likely not to have major postoperative complications. Therefore, we define a low perceived cardiorespiratory fitness as a VSAQ score $\leq 7$ METs.

Patients with a VSAQ score $\leq 7$ METs will receive very limited information about the study. They will be asked to participate in a study addressing the role of cardiorespiratory fitness (assessed with a CPET) on postoperative complications following colorectal resection. If informed consent is obtained (Appendix 6.1), the patient will be randomized to the intervention group (prehabilitation) or the usual care group, and they will undergo a CPET to obtain objective information about their cardiorespiratory fitness, as standard workup. Eventually, patients with a VAT $<11 \mathrm{~mL} / \mathrm{kg} / \mathrm{min}$ can participate in the study, whereas randomized patients with a VAT $\geq 11 \mathrm{~mL} / \mathrm{kg} / \mathrm{min}$ cannot participate in the study (in the intervention group, nor in the usual care group). See Figure 1 for a flow diagram of the study design.

Patients in the prehabilitation group and the usual care group will be informed differently. The patients in the prehabilitation group will be fully informed about the study in a patient 
information letter that explains the aims and expectations of this study, as well as the risks and benefits of participating. Patients in the usual care group will not receive information about the possible effects of prehabilitation to avoid the risk that these patients will initiate preoperative physical exercise training themselves. The usual care group will receive a patient information letter about the registration of perioperative data and the hypothesized relation of cardiorespiratory fitness with postoperative complications after colorectal resection. Patients in both the prehabilitation group and the usual care group will be contacted by the clinical research coordinator a few days later to answer their questions, and to inform them about the CPET date. This procedure is approved by the Medical Ethics Committee Twente.

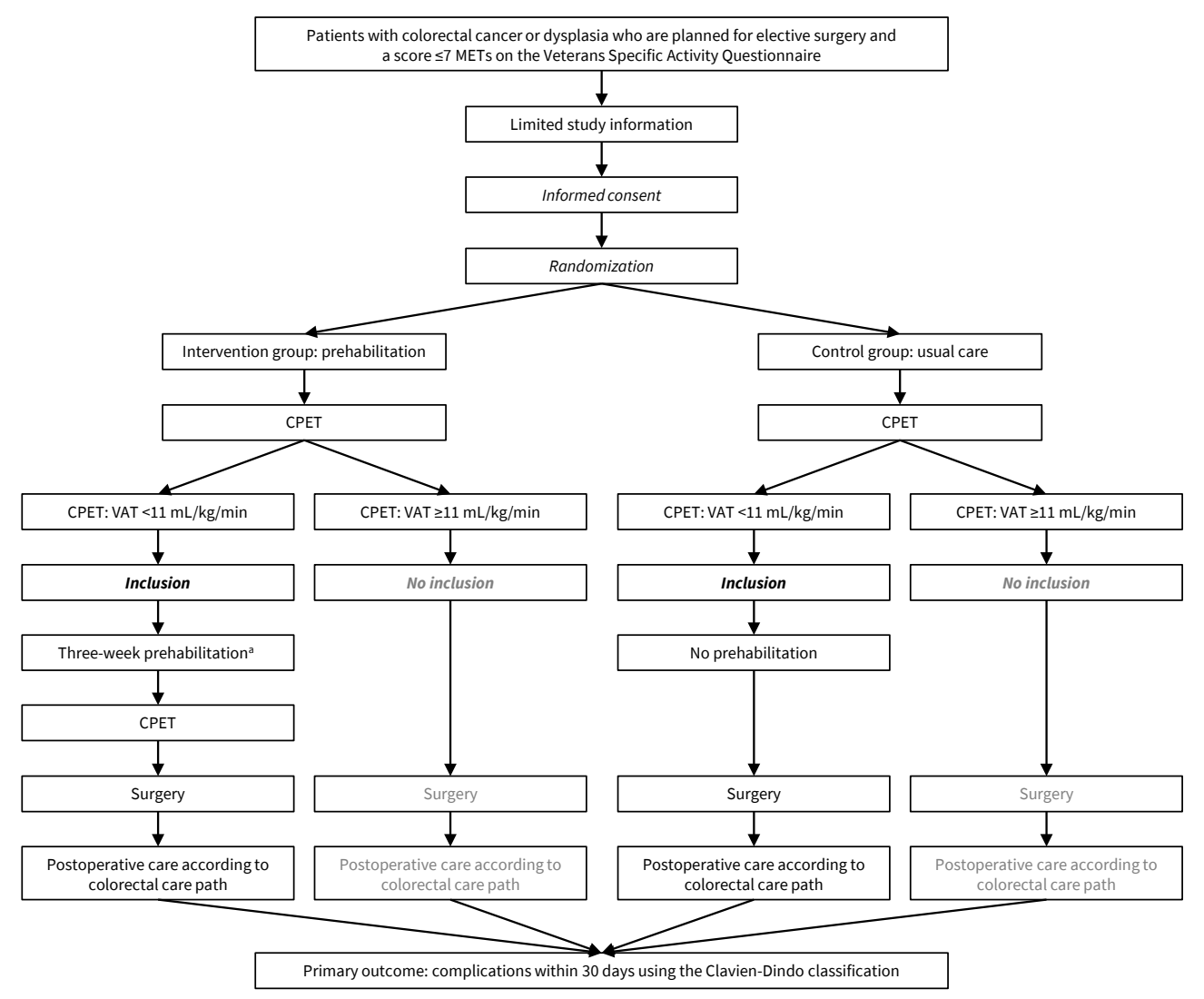

Figure 1 Flow diagram of the study design.

a: Patients with colon cancer or dysplasia grade I, II, or III will participate in the prehabilitation program in the time between contemplation of surgery and the procedure, whereas patients with rectal cancer, in case neoadjuvant therapy is needed, will complete the prehabilitation program prior to radiotherapy (in case of neoadjuvant radiotherapy, 5x5 Gy) or in the twelve-week period after neoadjuvant chemoradiotherapy (in week 10-12).

Abbreviations: CPET, cardiopulmonary exercise test; MET, metabolic equivalent of task; VAT, ventilatory anaerobic threshold. 


\section{Randomization}

To analyze the effect of a three-week prehabilitation program on postoperative complications, patients with a VSAQ score $\leq 7$ will be randomized in the intervention group (prehabilitation) or the control group (usual care) by using block-stratified randomization. ${ }^{37}$ Randomization is stratified by disease and treatment type: 1) patients with colon cancer, 2) patients with rectal cancer who will receive one week of neoadjuvant radiotherapy, and 3) patients with rectal cancer who will receive five weeks of neoadjuvant chemoradiotherapy. The clinical research coordinator of the surgical department will be responsible for the randomization. Participants, care providers, and outcome assessors will not be blinded after randomization. However, the data analysts will be blinded to group allocation and are independent to the intervention. After randomization, all participants will perform a CPET for final inclusion, as merely patients with a VAT $<11 \mathrm{~mL} /$ $\mathrm{kg} / \mathrm{min}$ can participate in the study.

\section{Interventions}

\section{Prehabilitation program}

Patients in the prehabilitation group will participate in a three-week (three sessions per week, nine sessions in total) supervised physical exercise training program prior to colorectal resection. The prehabilitation program is based on the program developed by Dunne et al. ${ }^{25,28}$ which has been shown to be feasible in sedentary healthy volunteers, ${ }^{28}$ and later demonstrated to deliver improvements in CPET scores and perceived health-related quality of life prior to hepatic resection. ${ }^{25}$ In the studies of Dunne et al. ${ }^{25,28}$ prehabilitation consisted of twelve interval training sessions over a four-week period, whereas in the present study patients will perform nine preoperative sessions of individually-tailored interval training (personalized prehabilitation) over a three-week period. In the literature, it has been shown that as little as six sessions of 'all-out' high-intensity interval training over two weeks is sufficient to enhance cardiorespiratory fitness and improve skeletal muscle oxidative capacity in untrained and recreationally active individuals. ${ }^{38,39}$ However, a comparison with our patients with colorectal cancer and a low cardiorespiratory fitness cannot be made, because their 'all-out' exercise program differs from our exercise intervention, as 'all-out' exercise may not be safe, tolerable, or appealing for our patient group.

Patients will prehabilitate on Monday, Wednesday, and Friday, or on Tuesday, Thursday, and Saturday to improve their physical fitness preoperatively. Each 60-minute training session consists of moderate-to-high intensity interval training to improve cardiorespiratory fitness, and resistance training to improve peripheral muscle strength (see Tables 1 and 2). The interval training program will be tailored to each individual patient, based on the results of the CPET. The training sessions will be supervised by a selected group of trained physical therapists in community physical therapy practices 
in the adherence area of the hospitals. Additionally, the patient performs unsupervised home exercises at a moderate exercise intensity (e.g., walking, cycling, or stair climbing) twice a week for at least 30 minutes, as checked by the physical therapist. We added these functional exercises to the prehabilitation program as de Vreede et al. ${ }^{40}$ demonstrated that functional task exercises are effective at improving functional task performance in healthy elderly women. Hence, home-based functional exercises that are of relevance for a patient may have an important role in helping patients to mobilize quickly postoperatively, to be physically active throughout the hospitalization period postoperatively, and to maintain independent physical functioning. Indeed, prehabilitation ideally should not focus merely on the preoperative period. Postoperatively, hospital culture and infrastructure should stimulate the patient to be physically active. However, in most hospitals, health care is entirely organized around the patient's bed, ${ }^{41}$ which invites patients to lie in bed, even without a medical reason. Bed-centered care probably also hampers the effectiveness of prehabilitation to improve postoperative outcomes.

Table 1 Content of the three-week interval training program.

\begin{tabular}{|c|c|c|}
\hline & Duration & Intensitya \\
\hline Warm-up & 7 minutes & $50 \%$ \\
\hline Interval session & 30 minutes & $\begin{array}{l}\text { Work interval: } 120 \% \\
\text { Recovery interval: } 50 \%\end{array}$ \\
\hline Week 1 , session 1,2 , and 3 & $\begin{array}{l}\text { Work interval: } 120 \text { seconds } \\
\text { Recovery interval: } 180 \text { seconds }\end{array}$ & \\
\hline Week 2 , session 4,5 , and 6 & $\begin{array}{l}\text { Work interval: } 140 \text { seconds } \\
\text { Recovery interval: } 160 \text { seconds }\end{array}$ & \\
\hline Week 3 , session 7,8 , and 9 & $\begin{array}{l}\text { Work interval: } 160 \text { seconds } \\
\text { Recovery interval } 140 \text { seconds }\end{array}$ & \\
\hline Cool-down & 3 minutes & $60 \%$ \\
\hline
\end{tabular}

a: Expressed as a percentage of the individually achieved work rate (in Watt) at the ventilatory anaerobic threshold obtained from the cardiopulmonary exercise test.

Patients with colon cancer or dysplasia will participate in the prehabilitation program in the time between contemplation of surgery and the procedure, whereas patients with rectal cancer, in case neoadjuvant therapy is needed, will complete the physical exercise training program prior to radiotherapy (in case of neoadjuvant radiotherapy, 5x5 Gy) or in the twelve-week period after neoadjuvant chemoradiotherapy (training in the last three weeks before surgery). 
Table 2 Content of the three-week peripheral resistance training program ${ }^{\mathrm{a}}$.

\begin{tabular}{llll}
\hline & Repetitions $^{\mathbf{b}}$ & Duration & Intensity $^{\mathbf{c}}$ \\
\hline Week 1, session 1, 2, and 3 & $3 \times 8$ repetitions & 20 minutes & $70 \%$ of 1RM \\
Week 2, session 4, 5, and 6 & $3 \times 8$ repetitions & 20 minutes & $76 \%$ of 1RM \\
Week 3, session 7, 8, and 9 & $3 \times 8$ repetitions & 20 minutes & $82 \%$ of 1RM \\
\hline
\end{tabular}

a: Peripheral resistance training of the large muscle groups of the lower and upper extremities using open and closed kinetic chain exercises (without physical therapy equipment or machines): crouching by means of squat exercises (primary muscle group: quadriceps femoris; secondary muscle groups: gluteal muscles, hamstring muscles, and gastrocnemius muscle), pulling by means of pulley exercises (primary muscle groups: latissimus dorsi muscle and rhomboid muscles; secondary muscle groups: biceps brachii muscle, rotator cuff, and trapezius muscle), pushing by means of pulley exercises (primary muscle group: pectoral muscles; secondary muscle groups: shoulder muscles, triceps brachii muscle), and lifting by means of pulley exercises (primary muscle groups: trunk muscles and shoulder muscles; secondary muscle groups: quadriceps femoris, gluteal muscles, and trapezius muscle)

b: Rest intervals of 60-90 seconds between each set of repetitions.

c: Based on the 10RM, the patient's 1RM was determined using the Oddvar Holten diagram.

Abbreviations: 1RM, one-repetition maximum; 10RM, ten-repetition maximum.

\section{Usual care group}

Patients in the control group receive usual care and will not participate in a prehabilitation program. In addition, no advice about physical exercise training is offered to them.

\section{Measurements}

Both the prehabilitation group and usual care group will participate in a series of outcome measure assessments at baseline, seven days postoperatively, and thirty days postoperatively, whereas the prehabilitation group will participate in a series of outcome measure assessments after the three-week prehabilitation program as well (see Table 3).

\section{Cardiopulmonary exercise test}

A CPET will be used at baseline to assess cardiorespiratory fitness, as indicated by the VAT, in order to select and thereupon advice/invite eligible patients to participate in the study. For patients in the prehabilitation group, a second CPET will be used to investigate the effect of the prehabilitation program on cardiorespiratory fitness (single-arm study design). Patients free from any absolute and/or relative exclusion criteria, as stated by the American Thoracic Society and American College of Chest Physicians position statement, ${ }^{42}$ will be able to perform the CPET. The following standardized pre-test instructions will be given to the patients: 1 ) consume the last (light) meal at least two hours before exercise testing, 2) adhere to usual use of medication, and 3) wear comfortable sporting clothes and shoes. The CPET will be performed under controlled conditions at the lung function department of both hospitals, using a calibrated electronically braked cycle ergometer (Ergoline, Ergoselect 100, Bitz, Germany at Medisch Spectrum Twente and Lode Excalibur Sport, Lode BV, Groningen, the Netherlands at Ziekenhuisgroep Twente). Seat height will be adjusted to the patient's leg length. During the CPET, patients breathe through a facemask (Hans Rudolph, Kansas City, MO, USA) connected to a Triple V volume transducer 
to perform breath-by-breath measurements of oxygen uptake, carbon dioxide production, respiratory flow, and volume parameters (Oxycon Pro, Jaeger, Hoechberg, Germany). In both hospitals the same respiratory gas analysis system will be used. Before each CPET at the Medisch Spectrum Twente and each morning at the Ziekenhuisgroep Twente, flowvolume calibration (three-litre syringe) and gas calibration (ambient air and a gas mixture of $16 \%$ oxygen and $5 \%$ carbon dioxide) will be performed, and ambient conditions are set. Twelve-lead electrocardiography will be used to continuously monitor heart rate during the test. Blood pressure (SunTech Tango+, SunTech Medical, Inc, Marrisville, NC, USA) will be automatically measured every three minutes to be aware of extreme hypertension or to detect hypotension. Oxygen saturation (Nonin 9600, Nonin Medical, Inc., Plymouth, $\mathrm{MN}, \mathrm{USA}$ ) is continuously recorded during the test by finger pulse oximetry.

After the test protocol is explained to the patient, the CPET starts with a two-minute rest period during which baseline cardiopulmonary data are collected. The patient then starts unloaded cycling at a pedaling frequency of $60-80$ rotations per minute for three minutes, where after the work rate will be increased every minute by 5 to 15 Watt, depending on the patient's physical fitness level and aimed at reaching a maximal effort within eight to twelve minutes. ${ }^{43}$ The CPET continues until the patient is unable to maintain a pedaling frequency above 60 rotations per minute due to volitional exhaustion and despite strong verbal encouragement. The CPET will be considered to be at or near the maximal level when the patient shows clinical signs of intense effort (e.g., unsteady biking, sweating, and clear unwillingness to continue exercising despite strong encouragement), when the patient is unable to maintain a pedaling frequency above 60 rotations per minute, and when at least one of the following criteria is met: a heart rate at peak exercise of $>95 \%$ of predicted (predicted peak heart rate [beats $/ \mathrm{min}$ ] $=208-0.7 \times$ age [years]) or a respiratory exchange ratio at peak exercise of $>1.10$. After termination of the CPET, the patient will complete a recovery phase of unloaded cycling at a low pedaling frequency for five minutes. A clear description of adverse events and early test termination will be given.

During the CPET, absolute values at peak exercise are calculated as the average value over the last 30 seconds prior to termination of the test. Peak heart rate is defined as the highest heart rate achieved during the CPET. Cardiorespiratory fitness will be assessed by determining the VAT, by evaluating the peak oxygen uptake in case the patient performed a maximal effort, and by calculating the oxygen uptake efficiency slope. ${ }^{44}$ The VAT will be detected by using the V-slope method, which is based on analyzing the slopes of oxygen uptake and carbon dioxide production. The VAT can be determined by using computerized regression analysis of these slopes. The point at which the linear slope of the relation between the carbon dioxide production and oxygen uptake changes, is called the VAT. ${ }^{45}$ When the V-slope is expected to be unreliable, the ventilatory equivalents will be used to determine the VAT. ${ }^{46}$ In this ventilatory equivalents method, the VAT will be defined 


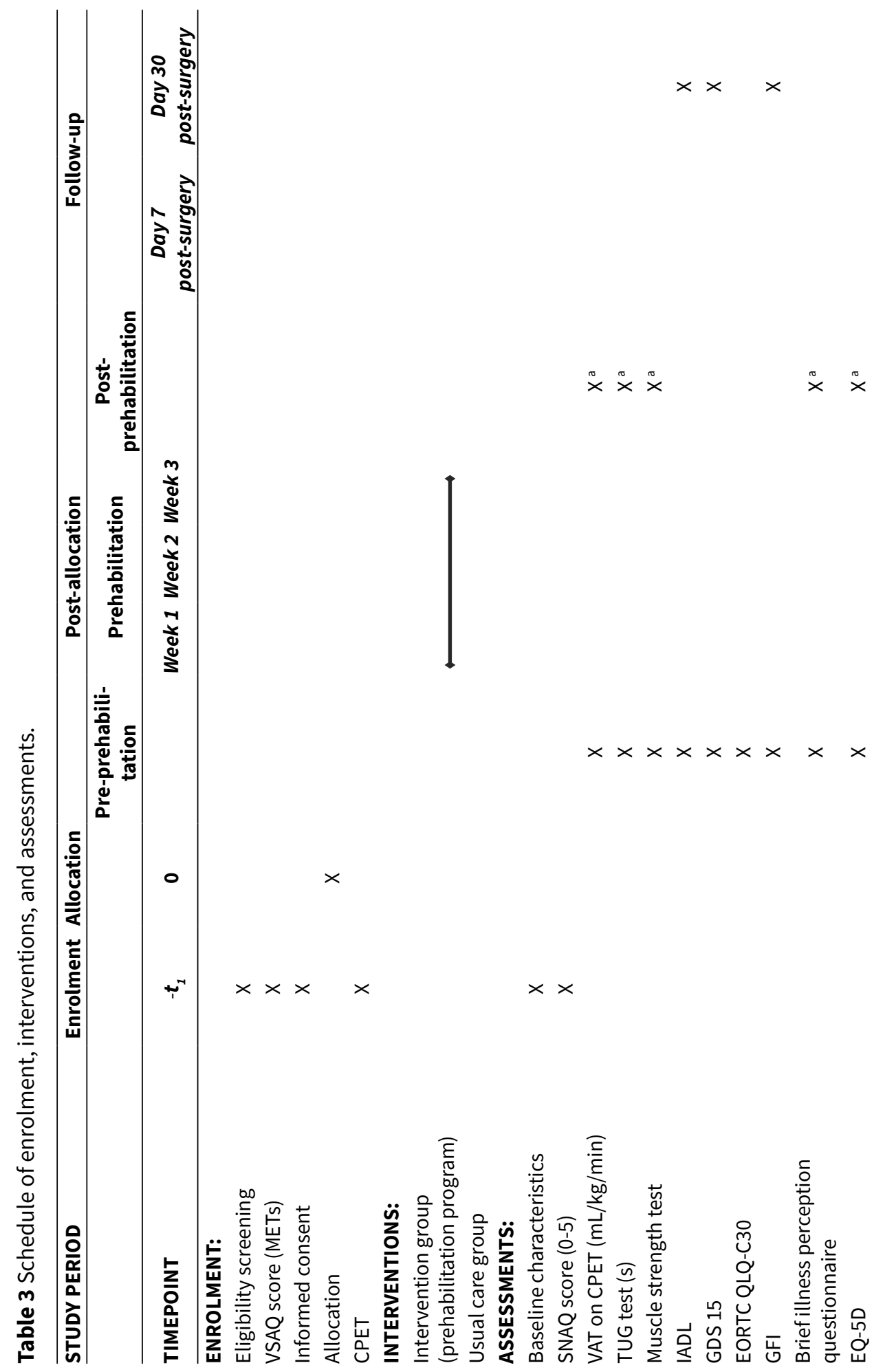




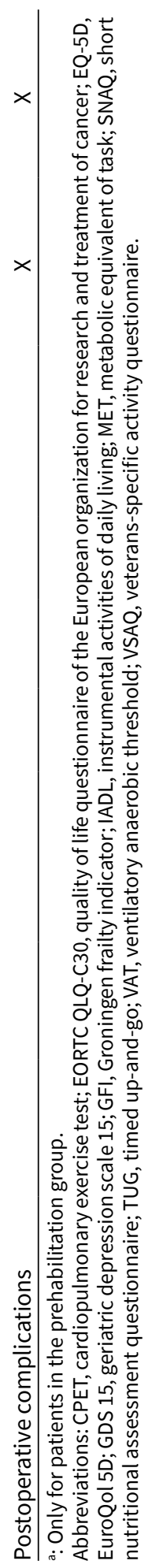


as the point at which the ventilatory equivalent for oxygen and the partial end-tidal oxygen tension reached a minimum and thereafter began to rise in a consistent manner, coinciding with an unchanged ventilatory equivalent for carbon dioxide and partial endtidal carbon dioxide tension. ${ }^{42}$ The inter-observer variability in a preoperatively measured VAT has been reported to be acceptable for experienced clinicians, ${ }^{7}$ and DeCato et al. ${ }^{47}$ recently reported short-term repeatability for CPET parameters in healthy participants in a similar test protocol, and they found no evidence for a learning effect and no significant differences in variability related to sex, age, fitness level, and diurnal factors.

\section{Muscle strength}

To investigate the effect of the prehabilitation program on muscle strength, a trained physical therapist will measure handgrip strength and quadriceps strength at baseline for both groups and after three weeks of prehabilitation for the prehabilitation group.

Handgrip strength (recorded in kilograms) will be assessed using the Jamar dynamometer (Sammons Preston, Rolyan, Bolingbrook, IL, USA)..$^{48}$ Handgrip strength has shown to be an indicator of skeletal muscle mass and a predictor of the risk of postoperative complications. ${ }^{49,50}$ The test will be performed in upright position with the elbows stretched in a straight line downwards, starting with the dominant hand. First, the handle position, in which the patient has maximum strength, will be determined. Patients will be asked to squeeze the handle as forcefully as possible for about two seconds. Hereafter, two more measurements will be performed (three grip measurements per hand). Each measurement will be followed by a pause of $15-20$ seconds. Mathiowetz ${ }^{48}$ compared the Jamar and Rolyan hydraulic dynamometers for measuring grip strength and showed that the Jamar and Rolyan dynamometers have acceptable concurrent validity with known weights, excellent inter-instrument reliability, and strong concurrent validity.

Quadriceps strength (recorded in Newton) will be measured with a hand-held dynamometer (MicroFET2, Hoggan Health Industries Inc., West Jordan, UT, USA), which has been shown to be a feasible, inexpensive, and portable test of the quadriceps muscle strength for use in healthy older people. ${ }^{51}$ Patients will be seated at the examination table with their lower limbs bent over the edge (knees and hips flexed at $90^{\circ}$ ). If necessary, additional stabilization will be provided at the patients' shoulders by a second examiner. The test will be performed three times per leg, starting with the dominant leg. The handheld dynamometer will be placed on the anterior aspect of the tibia at the level of the malleoli. The physical therapist will give resistance in the opposite direction. The patient must hold the leg in place. The resistance will gradually be increased in six seconds till maximum resistance/strength. The measurement will be stopped when the patient moves his leg. Each measurement will be followed by a pause of 30 seconds. 


\section{Functional mobility}

To test (changes in) functional mobility, the physical therapist will execute the timed upand-go (TUG) test at baseline for both groups and after three weeks of training for the prehabilitation group. The TUG test, a reliable and valid test, measures the time (recorded in seconds) a patient needs to stand up from a standard arm chair (approximate seat height of $46 \mathrm{~cm}$ ), walk three meters, turn, walk back to the chair, and sit down again..$^{52,53}$ The patient will perform the test once before being timed, in order to become familiar with the test and therefore minimize the learning effect.

In the literature, there are a few studies that demonstrated the use of the TUG test to identify patients at risk for impaired postoperative outcome..$^{52,54,55}$ Huisman et al..$^{55}$ showed that the TUG test was an independent predictor of the occurrence of major complications in a onco-geriatric surgical population. In this study, the predictive value of the TUG test on postoperative outcome will be determined. The TUG test is easy to perform, cheap, and does not need special equipment and might therefore be an alternative for the CPET.

\section{Nutritional status}

Assessment of nutritional status using the short nutritional assessment questionnaire (SNAQ) score ${ }^{56}$ is part of usual care for our patients undergoing colorectal resection and is evaluated at baseline for both the intervention and usual care group. The SNAQ is an easy, short, valid, and reproducible questionnaire for early detection of malnutrition. ${ }^{56}$ Patients with a SNAQ score of 2 require nutritional support, whereas patients with a SNAQ score $\geq 3$ require supervision by a dietician and nutritional support. In this study the SNAQ score will be used as a baseline characteristic.

\section{American Society of Anesthesiologists score}

The American Society of Anesthesiologists (ASA) score is the most widely used system to describe a patient's preoperative health status. The ASA score has five classes ranging from class I, a completely healthy patient, to class V, a moribund patient who is not expected to live 24 hours with or without surgery. ${ }^{57,58}$ The ASA score will be assessed at baseline for both groups.

\section{World Health Organization performance scale}

The World Health Organization (WHO) performance scale is a tool to assess how a patient's disease is progressing, how it affects activities of daily life, and it helps the clinician to determine appropriate treatment and prognosis. ${ }^{57}$ The WHO performance scale will be assessed at baseline in the prehabilitation group and in the usual care group.

\section{Charlson comorbidity index}

The Charlson comorbidity index ${ }^{59,60}$ will be scored at baseline for the prehabilitation group 
and usual care group. It can be a useful preoperative tool in predicting morbidity and mortality outcomes in patients with colorectal cancer. $^{61}$

\section{Questionnaires}

Every patient will visit an oncology nurse prior to colorectal resection, which is part of usual care. The oncology nurse will ask the patient to fill out several questionnaires: the instrumental activities of daily living (IADL), ${ }^{62-64}$ geriatric depression scale 15 (GDS 15), ${ }^{65,66}$ the quality of life questionnaire of the European organization for research and treatment of cancer (EORTC QLQ-C30), ${ }^{67,68}$ and the Groningen frailty indicator (GFI). ${ }^{69}$ The IADL will investigate whether a patient experiences difficulties in carrying out instrumental activities that are essential to independent living. The GDS 15 will be used to measure perceived depressive symptoms or emotional health, and has good reliability, validity, sensitivity, and specificity for older people. ${ }^{70}$ Patients will answer 'yes' or 'no' to fifteen questions depending on how they have felt over the past week. A score of 0-4 indicates normal mood or emotional health status, 5-9 indicates mild depression, and 10-15 indicates moderateto-severe depression. The EORTC QLQ-C30 is a reliable and valid measure of the perceived health-related quality of life of patients diagnosed with cancer in multicultural clinical research settings. ${ }^{67}$ The GFI is a short and easy-to-use instrument to measure frailty, which is defined as a loss of resources in several domains of functioning, which leads to a declining reserve capacity for dealing with stressors. ${ }^{69}$ The patient will be asked to fill out the IADL, GDS 15, and GFI for the second time thirty days after surgery in order to investigate the effect of surgery on the IADL, depression, quality of life, and frailty, in both the prehabilitation and usual care group.

A limited geriatric assessment will be performed at baseline for the prehabilitation group and the usual care group, measuring comorbidity with the Charlson comorbidity index, signs of depression using the GDS 15, and functional status using the IADL. We will investigate the value of the limited geriatric assessment to predict postoperative complications. In addition, the GFI will be used in order to examine whether a screening tool can replace this limited geriatric assessment.

Illness perception will be recorded in both groups by using the brief illness perception questionnaire, a quick and easy to use questionnaire. ${ }^{71}$ The face and content properties were found to be acceptable, and the reproducibility showed moderate-to-good reliability ${ }^{71}$. Illness perception is an important factor of health. Patients' personal thoughts about the symptoms they experience can be seen as one of the psychosocial factors by which variance in physical functioning in patients can be explained. ${ }^{71}$ The physical therapist will ask patients in the prehabilitation group to fill out the brief illness perception questionnaire again after the three-week training period, in order to investigate the effect of the prehabilitation program on illness perception. 
Health status will be evaluated by using the EuroQol 5D (EQ-5D). ${ }^{72}$ The EQ-5D questionnaire is a short profile measure of perceived health-related quality of life, and has five domains: 1) perceived mobility, 2) self-care, 3) usual activities, 4) pain/discomfort, and 5) anxiety/ depression. Each domain is scored by patients according to three levels (no problems, some problems, extreme problems), for which quality weights (to enable calculation of quality adjusted life years for use in economic evaluations) are available. ${ }^{73}$ The EQ-5D is widely used in oncology and it has shown a reasonable degree of reliability, content validity, construct validity, and responsiveness in the majority of available studies. ${ }^{74}$ The physical therapist will ask patients in the prehabilitation group to fill out the EQ-5D again after the three-week training period, in order to investigate the effect of the prehabilitation program on health status.

\section{Study outcomes}

\section{Primary study parameter}

The primary study parameter is the number of overall postoperative complications within thirty days after surgery in the prehabilitation group and usual care group. Complications will be divided in surgical and non-surgical complications. Surgical complications will be scored as anastomotic leakage, perineal wound complication, rectal stump abscess, intra-abdominal abscess, fistula, sepsis, ileus, abdominal wound complication, intestinal necrosis, stoma complication, urological complication, bleeding, iatrogenic intestinal injury, or iatrogenic vascular injury. Non-surgical complications will be scored as cardiovascular, pulmonary, thromboembolic, renal, or neurological. Complications will be recorded and graded by using the Clavien-Dindo classification. ${ }^{75,76}$ Readmissions within thirty days will also be recorded.

\section{Secondary study parameters}

Secondary study parameters are 1) cardiorespiratory fitness, as indicated by the VAT, after the prehabilitation program in the intervention group just before surgery, 2) length of stay, 3) postoperative complications within thirty days after surgery in patients with a preoperative VAT $\geq 11 \mathrm{~mL} / \mathrm{kg} / \mathrm{min}$ and who will therefore not be included in the randomized controlled trial compared to patients in the prehabilitation or usual care group (for the prehabilitation group, we will use the post-prehabilitation CPET data), 4) the value of a limited geriatric assessment in this patient group to predict (in combination with VAT) postoperative complications, and 5) cost-effectiveness of prehabilitation.

\section{Other study parameters}

Preoperative factors that could be associated with postoperative complications will be recorded, these include: age, sex, body height, body mass, body mass index, nutritional status, smoking status, location and type of the tumor, presence or absence of metastases, CPET parameters other than the VAT, WHO performance score, ASA score, ${ }^{77}$ Charlson 
comorbidity index,${ }^{59}$ type of surgical resection, muscle strength, and the TUG test.

\section{Safety}

All adverse events reported spontaneously by participating patients or observed by the investigator or his staff will be recorded.

\section{Data analysis}

Sample size calculation

Nowadays, there is $33 \%$ morbidity and $3.8 \%$ mortality in patients undergoing colorectal surgery in the Netherlands. ${ }^{3,78}$ We expect the high-risk group (patients with a VAT $<11 \mathrm{~mL}$ / $\mathrm{kg} / \mathrm{min}$ ) to have a morbidity rate of $50 \%$. Dunne et al. stated that a $10 \%$ improvement in cardiorespiratory fitness would move $30 \%$ of the patients from high to low operative risk. ${ }^{25,28}$ Robinson et al. ${ }^{34}$ showed that $21 \%$ of the non-frail colorectal patients had a postoperative complication, versus $40 \%$ in the pre-frail group and $58 \%$ in frail patients. We hypothesized a complication rate of $20 \%$ in the prehabilitation group.

For the sample size calculation we used the computer program PS Power and Sample Size Calculations version 3.0, January 2009 (Copyright $\odot 1997-2009$ by William D. Dupont and Walton D. Plummer). ${ }^{79,80}$ Using an alpha of 0.0492 (due to one interim analysis) and a power of 0.80 , we need 39 patients in each group to find statistically significant results. Taking into account 10\% drop out, we need 43 patients in each group.

\section{Procedures for data checking and entering}

Data (coded on study code) will be entered in the Statistical Package for the Social Sciences for Windows (version 23.0; IBM, SPSS Inc., Chicago, IL, USA). All data are held in an encrypted format and will be stored in a secured locked room. All variables will be checked for the number of missing, impossible or improbable values, prior to statistical analysis. In case of improbable or impossible values, the patient's data file will be checked by the data manager. Descriptive statistics will be calculated for all variables, and distributional assumptions will be checked. The study coordinator, chief investigator, and a blinded data analyst will have access to the final trial dataset. Due to the low-risk nature of the intervention, the Medical Ethics Committee determined that a data monitoring committee is not required.

\section{Statistical analysis}

Nominal variables will be presented as numbers with percentages. Continuous variables will be presented as mean $\pm S D$, or as median and interquartile range, as appropriate. Data will be presented in tables and figures. All tests will be performed on the intention to treat population, and on the per-protocol population. Data distribution will be checked with help of an epidemiologist. 
For categorical variables, chi-squared tests or Fisher's Exact tests, as appropriate, will be performed to analyze the difference between the intervention and usual care group. For continuous variables this will be done by independent samples t-tests or Mann-Whitney $\mathrm{U}$ tests, as appropriate. A repeated measurements analysis (mixed models in SPSS) will be performed to assess changes over time in continuous variables. A cost-effectiveness analysis will be performed, with the use of quality adjusted life years. Withdrawn patients will be followed by checking the patient's data file. We will use the intention to treat analysis to avoid various misleading artifacts.

When half of the patients needed that are calculated (43 patients) are included, a blinded independent data analyst will perform an interim analysis with a stopping rule according to O'Brien-Fleming. ${ }^{81}$ If this interim analysis shows a significant difference in postoperative complications between the prehabilitation group and the usual care group (at $p<0.0054$ ), this data analyst will contact the Medical Ethics Committee, and debate the outcome of the interim analysis and its eventual repercussions. Thereupon this committee will advise the principal investigator of the research team (JK) to decide to stop the inclusion in case of superiority. If the interim analysis shows no or minimal difference $(<10 \%$ on overall complications), the study will be stopped due to futility. Because of the interim analysis, the final $P$-value that is considered to be significant is reduced to $p<0.0492$.

\section{Discussion}

Surgery is an important treatment modality in patients with colorectal cancer. Unfortunately, over $30 \%$ of patients develop a postoperative complication after elective colorectal resection. ${ }^{3}$ Older patients, especially frail patients, with a poor cardiorespiratory fitness are more prone to postoperative complications and require specific preoperative risk stratification. Optimizing preoperative physical fitness (e.g., cardiorespiratory fitness, muscle strength, and functional mobility) through prehabilitation may improve postoperative outcomes in patients undergoing major abdominal surgery. ${ }^{6}$ Currently, there is a lack of information in the literature about the effect of preoperative exercise training on overall postoperative complications in high-risk older patients with colorectal cancer. Most of the studies so far were rather underpowered, heterogeneous, and biased towards selection of patients with low risk of postoperative complications. ${ }^{6,29,30}$ The primary aim of our current randomized controlled trial is to measure the effect of a threeweek prehabilitation program on postoperative complications in patients with a poor cardiorespiratory fitness, as indicated by a VAT $<11 \mathrm{~mL} / \mathrm{kg} / \mathrm{min}$, who will undergo elective colorectal resection for colorectal cancer or dysplasia grade I, II, or III.

Postoperative complications can extend length of hospital stay, and may lead to 
repeated hospital admissions, chronic ill health, and a decrease or even permanent loss in functional capacity and health-related quality of life. ${ }^{6}$ Patients with a higher cardiorespiratory fitness might be more resilient to cope with the stress associated with major abdominal surgery, with subsequent better postoperative outcomes. Optimizing preoperative cardiorespiratory fitness may therefore not only decrease postoperative complications, but may also decrease associated hospital costs, enhance the patient's quality of life, and promote independent functioning in daily life. Our intervention fits well with the 'prevention of limitations to function' view in the elderly, of the Health Council of the Netherlands, ${ }^{82}$ as well as with the recently proposed new schemes of the international classification of functioning, disability, and health (ICF) to promote functioning and health. ${ }^{83}$

According to the Dutch guidelines, patients will ideally be operated within five weeks from time of pathologic-anatomic diagnosis, and at latest within seven weeks. ${ }^{3}$ The period between the decision for surgery and the actual surgery is limited, and therefore the valuable available time for prehabilitation is short. Moreover, some patients experience (severe) complaints of their illness, and therefore resection cannot be postponed for several weeks. However, given the need for prehabilitation interventions to be both effective and time-efficient, Weston et al. ${ }^{84}$ indicated that carefully designed and supervised high-intensity interval training programs might be a promising perioperative strategy for enhancing cardiorespiratory fitness within a short period.

The present study, like all studies do, bares some limitations. Firstly, merely patients in the prehabilitation group will perform a second CPET to investigate the effect of the prehabilitation program on cardiorespiratory fitness. Consequently, we are not able to investigate the changes in cardiorespiratory fitness in the preoperative waiting period in the usual care group. However, the waiting period in patients in the usual care group was kept as short as possible to prevent these patients to improve their level of fitness by organizing their own preoperative exercise training program. Moreover, a study in patients planned for elective hepatic resection for colorectal liver metastases reported no significant differences in CPET variables in the control group after a four-week waiting period. ${ }^{25}$ Secondly, the prehabilitation program in the present study consists only of a preoperative exercise training program, while it was recently indicated that studies evaluating a prehabilitation program combined with a nutritional intervention before elective major surgery in adults are producing encouraging early results. ${ }^{31}$ However, definitive clinical evidence of physical and nutritional prehabilitation is currently very limited. ${ }^{31,85,86}$ Thirdly, up till now, patient inclusion appears to be challenging. Not all patients are able and/or willing to attend the prehabilitation program due to personal, logistical, and time limitations (e.g., restricted time availability between the first outpatient visit and surgery, living too far away from the community physical therapy 
practice, and/or no traveling opportunities, planned vacation prior to surgery, not interested in participation). Moreover, at the start of the present study patients in both groups were informed about the possible effects of prehabilitation. We experienced that some patients in the usual care group initiated preoperative physical exercise training themselves, possibly resulting in the risk that we will not be able to detect a difference between the prehabilitation and usual care group. Therefore, we made an amendment to the study protocol (approved in April 2016 by the Medical Ethics Committee Twente) in which patients in the prehabilitation group and the usual care group will be informed differently. The patients in the usual care group will not receive information about the possible effects of prehabilitation and their elective colorectal resection was planned at the earliest convenience. Fourthly, in the present study a geriatric assessment will be completed at baseline only. Baseline data as predictors of postoperative outcome may be confounded by the effect of the prehabilitation program. Therefore, only data from patients in the usual care group will be used to investigate the value of a limited geriatric assessment to perform preoperative risk stratification. However, the power for this secondary objective might be inadequate, as the sample size calculation was not based on this objective.

We believe that preoperative exercise training in patients 'merely' undergoing elective colorectal resection will be only cost-effective and sustainably implementable in usual care pathways when performed in high-risk patients (those with a low cardiorespiratory fitness, e.g., a VAT $<11 \mathrm{~mL} / \mathrm{kg} / \mathrm{min}$ ). Hence, an adequate preoperative risk stratification protocol, such as the protocol used in the current study (a CPET for patients with a VSAQ score $\leq 7$ METs, of which those patients with a VAT $<11 \mathrm{~mL} / \mathrm{kg} / \mathrm{min}$ at the CPET will be eligible for and advised to participate in prehabilitation), will probably lead to a feasible sustainable implementation of the prehabilitation concept. For patients undergoing elective colorectal resection and (neo)adjuvant chemotherapy and/or radiotherapy, prehabilitation might be cost-effective in all patients. Cost-effectiveness should be expected, and therefore evaluated, in terms of, among other things, fewer postoperative complications, fewer reoperations, less intense clinical care, a shorter length of stay, a more effective surgical planning (process-optimization), fewer readmissions, less intense rehabilitation, shorter rehabilitation period, and earlier resumption of work.

The strengths of our present study are the selection of high-risk patients, the clear description of the study design and prehabilitation program, the 1:1 randomization between the prehabilitation and usual care group, the use of validated measurement instruments, and the adjustment of the physical exercise training program to the cardiorespiratory fitness of the individual patient based upon the results of the CPET. Moreover, prehabilitation will be performed in a community physical therapy practice in the catchment of the patient's home. In this way we will try to reach the frailest patients 
who are not capable to visit the hospital each time.

\section{Trial status}

The trial started patient inclusion in February 2014 and is expected to be completed by the end of 2018. 


\section{References}

1. Haggar FA, Boushey RP. Colorectal cancer epidemiology: incidence, mortality, survival, and risk factors. Clin Colon Rectal Surg 2009; 22(4): 191-7.

2. Integraal Kankercentrum Nederland (IKNL). Cijfers over kanker. February 4, 2016; Available at: http://cijfersoverkanker.nl/selecties/Dataset_1/img58245726017da. Accessed November 10, 2016.

3. Dutch Institute for Clinical Auditing (DICA) Leiden. DICA jaarrapportage 2014: Dutch Surgical Colorectal Audit. 2014; Available at: http://www.clinicalaudit.nl/jaarrapportage/2014/dsca. html. Accessed December 12, 2017.

4. Hulzebos EH, van Meeteren NL. Making the elderly fit for surgery. Br J Surg 2016; 103(4): 463.

5. Dutch Institute for Clinical Auditing (DICA) Leiden. DICA jaarrapportage 2016: Dutch Surgical Colorectal Audit. 2016; Available at: http://dica.nl/jaarrapportage-2016/dsca. Accessed December 12, 2017.

6. Moran J, Guinan E, McCormick P, et al. The ability of prehabilitation to influence postoperative outcome after intra-abdominal operation: A systematic review and meta-analysis. Surgery 2016; 160(5): 1189-1201.

7. Sinclair RC, Danjoux GR, Goodridge V, Batterham AM. Determination of the anaerobic threshold in the preoperative assessment clinic: inter-observer measurement error. Anaesthesia 2009; 64(11): 1192-5.

8. Kothmann E, Danjoux G, Owen SJ, Parry A, Turley AJ, Batterham AM. Reliability of the anaerobic threshold in cardiopulmonary exercise testing of patients with abdominal aortic aneurysms. Anaesthesia 2009; 64(1): 9-13.

9. Older P, Hall A. Clinical review: how to identify high-risk surgical patients. Crit Care $2004 ; \mathbf{8}(5)$ : 369-72.

10. Junejo MA, Mason JM, Sheen AJ, et al. Cardiopulmonary exercise testing for preoperative risk assessment before hepatic resection. Br J Surg 2012; 99(8): 1097-104.

11. Ausania F, Snowden CP, Prentis JM, et al. Effects of low cardiopulmonary reserve on pancreatic leak following pancreaticoduodenectomy. Br J Surg 2012; 99(9): 1290-4.

12. Hightower CE, Riedel BJ, Feig BW, et al. A pilot study evaluating predictors of postoperative outcomes after major abdominal surgery: Physiological capacity compared with the ASA physical status classification system. Br J Anaesth 2010; 104(4): 465-71.

13. Hartley RA, Pichel AC, Grant SW, et al. Preoperative cardiopulmonary exercise testing and risk of early mortality following abdominal aortic aneurysm repair. Br J Surg 2012; 99(11): 153946.

14. Snowden CP, Prentis JM, Anderson HL, Roberts DR, Randles D, Renton M, Manas DM. Submaximal cardiopulmonary exercise testing predicts complications and hospital length of stay in patients undergoing major elective surgery. Ann Surg 2010; 251(3): 535-41. 
15. Prentis JM, Manas DM, Trenell MI, Hudson M, Jones DJ, Snowden CP. Submaximal cardiopulmonary exercise testing predicts 90-day survival after liver transplantation. Liver Transpl 2012; 18(2): 152-9.

16. Prentis JM, Trenell MI, Jones DJ, Lees T, Clarke M, Snowden CP. Submaximal exercise testing predicts perioperative hospitalization after aortic aneurysm repair. J Vasc Surg 2012; 56(6): 1564-70.

17. Wilson RJ, Davies S, Yates D, Redman J, Stone M. Impaired functional capacity is associated with all-cause mortality after major elective intra-abdominal surgery. Br J Anaesth 2010; 105(3): 297-303.

18. Hennis PJ, Meale PM, Hurst RA, et al. Cardiopulmonary exercise testing predicts postoperative outcome in patients undergoing gastric bypass surgery. Br J Anaesth 2012; 109(4): 566-71.

19. Older P, Hall A, Hader R. Cardiopulmonary exercise testing as a screening test for perioperative management of major surgery in the elderly. Chest 1999; 116(2): 355-62.

20. Smith TB, Stonell C, Purkayastha S, Paraskevas P. Cardiopulmonary exercise testing as a risk assessment method in non cardio-pulmonary surgery: a systematic review. Anaesthesia 2009; 64(8): 883-93.

21. Chandrabalan VV, McMillan DC, Carter R, Kinsella J, McKay CJ, Carter CR, Dickson EJ. Preoperative cardiopulmonary exercise testing predicts adverse post-operative events and non-progression to adjuvant therapy after major pancreatic surgery. HPB (Oxford) 2013; 15(11): 899-907.

22. Older P, Smith R, Courtney P, Hone R. Preoperative evaluation of cardiac failure and ischemia in elderly patients by cardiopulmonary exercise testing. Chest 1993; 104(3): 701-4.

23. O'Doherty AF, West M, Jack S, Grocott MP. Preoperative aerobic exercise training in elective intra-cavity surgery: a systematic review. Br J Anaesth 2013; 110(5): 679-89.

24. Barberan-Garcia A, Ubre M, Roca J, et al. Personalized Prehabilitation in High-risk Patients Undergoing Elective Major Abdominal Surgery: A Randomized Blinded Controlled Trial. Ann Surg. 2018; 267(1): 50-56.

25. Dunne DF, Jack S, Jones RP, et al. Randomized clinical trial of prehabilitation before planned liver resection. Br J Surg 2016; 103(5): 504-12.

26. Jack S, West M, Grocott MP. Perioperative exercise training in elderly subjects. Best Pract Res Clin Anaesthesiol 2011; 25(3): 461-72.

27. Snowden CP, Prentis J, Jacques B, Anderson H, Manas D, Jones D, Trenell M. Cardiorespiratory fitness predicts mortality and hospital length of stay after major elective surgery in older people. Ann Surg 2013; 257(6): 999-1004.

28. Dunne DF, Jones RP, Poston GJ, Malik HZ, Palmer DH, Jack S, Fenwick SW. A prehabilitation program for liver surgery. HPB 2012; 14(Suppl. 2): 107-287.

29. Bruns ER, van den Heuvel $B$, Buskens $C J$, et al. The effects of physical prehabilitation in elderly patients undergoing colorectal surgery: a systematic review. Colorectal Dis 2016; 18(8): O26777. 
30. Hijazi Y, Gondal U, Aziz O. A systematic review of prehabilitation programs in abdominal cancer surgery. Int J Surg 2017; 39: 156-62.

31. West MA, Wischmeyer PE, Grocott MPW. Prehabilitation and Nutritional Support to Improve Perioperative Outcomes. Curr Anesthesiol Rep 2017; 7(4): 340-9.

32. Hoogeboom TJ, Oosting E, Vriezekolk JE, et al. Therapeutic validity and effectiveness of preoperative exercise on functional recovery after joint replacement: a systematic review and meta-analysis. PLoS One 2012; 7(5): e38031.

33. Pouwels S, Hageman D, Gommans LN, Willigendael EM, Nienhuijs SW, Scheltinga MR, Teijink JA. Preoperative exercise therapy in surgical care: a scoping review. J Clin Anesth 2016; 33: 476-90.

34. Robinson TN, Wu DS, Pointer L, Dunn CL, Cleveland JC,Jr, Moss M. Simple frailty score predicts postoperative complications across surgical specialties. Am J Surg 2013; 206(4): 544-50.

35. Myers J, Do D, Herbert W, Ribisl P, Froelicher VF. A nomogram to predict exercise capacity from a specific activity questionnaire and clinical data. Am J Cardiol 1994; 73(8): 591-6.

36. Ainsworth BE, Haskell WL, Leon AS, Jacobs DR,Jr, Montoye HJ, Sallis JF, Paffenbarger RS,Jr. Compendium of physical activities: classification of energy costs of human physical activities. Med Sci Sports Exerc 1993; 25(1): 71-80.

37. Press WH, Teukolsky S, Vetterling WT, Flannery B.P. Numerical Recipes in C: the Art of Scientific Computing. Cambridge University Press; 1992. p.280.

38. Gibala MJ, Jones AM. Physiological and performance adaptations to high-intensity interval training. Nestle Nutr Inst Workshop Ser 2013; 76: 51-60.

39. Gibala MJ, Little JP, Macdonald MJ, Hawley JA. Physiological adaptations to low-volume, high-intensity interval training in health and disease. J Physiol 2012; 590(5): 1077-84.

40. de Vreede PL, Samson MM, van Meeteren NL, Duursma SA, Verhaar HJ. Functional-task exercise versus resistance strength exercise to improve daily function in older women: a randomized, controlled trial. J Am Geriatr Soc 2005; 53(1): 2-10.

41. Punt IM, Bongers BC, van Beijsterveld CA, Hulzebos HJ, Dronkers JJ, van Meeteren NL. Surgery: moving people, improving outcomes. Geriatrics. Hyderabad, India: Avid Science. 2016. Chapter 1:2-30.

42. American Thoracic Society, American College of Chest Physicians. ATS/ACCP Statement on cardiopulmonary exercise testing. Am J Respir Crit Care Med 2003; 167(2): 211-77.

43. Wasserman K, Hansen JE, Sue DY, Casaburi R, Whipp BJ. Principles of exercise testing and interpretation, including pathophysiology and clinical applications. 3rd ed. USA: Lippincott Williams \& Wilkins. 2005.

44. Bongers BC, Berkel AE, Klaase JM, van Meeteren NL. An evaluation of the validity of the preoperative oxygen uptake efficiency slope as an indicator of cardiorespiratory fitness in elderly patients scheduled for major colorectal surgery. Anaesthesia 2017; 72(10): 1206-16.

45. Beaver WL, Wasserman K, Whipp BJ. A new method for detecting anaerobic threshold by gas exchange. J Appl Physiol (1985) 1986; 60(6): 2020-7.

46. Borley NR, Achan V. Respiratory physiology. Instant physiology. Blackwell Science; 2000. 
47. DeCato TW, Bradley SM, Wilson EL, Hegewald MJ. Repeatability and Meaningful Change of CPET Parameters in Normal Subjects. Med Sci Sports Exerc 2018; 50(3): 589-595.

48. Mathiowetz V. Comparison of Rolyan and Jamar dynamometers for measuring grip strength. Occup Ther Int 2002; 9(3): 201-9.

49. Mahalakshmi VN, Ananthakrishnan N, Kate V, Sahai A, Trakroo M. Handgrip strength and endurance as a predictor of postoperative morbidity in surgical patients: can it serve as a simple bedside test? Int Surg 2004; 89(2): 115-21.

50. Kerr A, Syddall HE, Cooper C, Turner GF, Briggs RS, Sayer AA. Does admission grip strength predict length of stay in hospitalised older patients? Age Ageing 2006; 35(1): 82-4.

51. Martin HJ, Yule V, Syddall HE, Dennison EM, Cooper C, Aihie Sayer A. Is hand-held dynamometry useful for the measurement of quadriceps strength in older people? A comparison with the gold standard Bodex dynamometry. Gerontology 2006; 52(3): 154-9.

52. Podsiadlo D, Richardson S. The timed "Up \& Go": a test of basic functional mobility for frail elderly persons. J Am Geriatr Soc 1991; 39(2): 142-8.

53. Mathias S, Nayak US, Isaacs B. Balance in elderly patients: the "get-up and go" test. Arch Phys Med Rehabil 1986; 67(6): 387-9.

54. Robinson TN, Wallace JI, Wu DS, et al. Accumulated frailty characteristics predict postoperative discharge institutionalization in the geriatric patient. J Am Coll Surg 2011; 213(1): 37,42; discussion 42-4.

55. Huisman MG, van Leeuwen BL, Ugolini G, et al. "Timed Up \& Go": a screening tool for predicting 30-day morbidity in onco-geriatric surgical patients? A multicenter cohort study. PLoS One 2014; 9(1): e86863.

56. Kruizenga HM, Seidell JC, de Vet HC, Wierdsma NJ, van Bokhorst-de van der Schueren,M.A. Development and validation of a hospital screening tool for malnutrition: the short nutritional assessment questionnaire (SNAQ). Clin Nutr 2005; 24(1): 75-82.

57. Oken MM, Creech RH, Tormey DC, Horton J, Davis TE, McFadden ET, Carbone PP. Toxicity and response criteria of the Eastern Cooperative Oncology Group. Am J Clin Oncol 1982; 5(6): 64955.

58. Dripps RD, Lamont A, Eckenhoff JE. The role of anesthesia in surgical mortality. JAMA 1961; 178: 261-6.

59. Charlson ME, Pompei P, Ales KL, Mackenzie CR. A new method of classifying prognostic comorbidity in longitudinal studies: development and validation. J Chronic Dis 1987; 40(5): 373-83.

60. Barnett S, Moonesinghe SR. Clinical risk scores to guide perioperative management. Postgrad Med J 2011; 87(1030): 535-41.

61. Ouellette JR, Small DG, Termuhlen PM. Evaluation of Charlson-Age Comorbidity Index as predictor of morbidity and mortality in patients with colorectal carcinoma. J Gastrointest Surg 2004; 8(8): 1061-7.

62. Lawton MP, Brody EM. Assessment of older people: self-maintaining and instrumental activities of daily living. Gerontologist 1969; 9(3): 179-86. 
63. Lawton MP, Moss M, Fulcomer M, Kleban MH. A research and service oriented multilevel assessment instrument. J Gerontol 1982; 37(1): 91-9.

64. Lawton MP. Scales to measure competence in everyday activities. Psychopharmacol Bull 1988; 24(4): 609-14.

65. Sheikh JI, Yesavage JA. Geriatric depression scale (GDS): Recent evidence and development of a shorter version. Clin Gerontol 1986; 5: 165-73.

66. Yesavage JA, Brink TL, Rose TL, Lum O, Huang V, Adey M, Leirer VO. Development and validation of a geriatric depression screening scale: a preliminary report. J Psychiatr Res 1982; 17(1): 37-49.

67. Aaronson NK, Ahmedzai S, Bergman B, et al. The European Organization for Research and Treatment of Cancer QLQ-C30: a quality-of-life instrument for use in international clinical trials in oncology. J Natl Cancer Inst 1993; 85(5): 365-76.

68. Fayers P, Bottomley A, EORTC Quality of Life Group, Quality of Life Unit. Quality of life research within the EORTC-the EORTC QLQ-C30. European Organisation for Research and Treatment of Cancer. Eur J Cancer 2002; 38(Suppl 4): S125-33.

69. Schuurmans H, Steverink N, Lindenberg S, Frieswijk N, Slaets JP. Old or frail: what tells us more? J Gerontol A Biol Sci Med Sci 2004; 59(9): M962-5.

70. McDowell I, Newell C. Measuring health: A guide to rating scales and questionnaires. (Second ed.) 1996. New York: Oxford University Press.

71. de Raaij EJ, Schroder C, Maissan FJ, Pool JJ, Wittink H. Cross-cultural adaptation and measurement properties of the Brief Illness Perception Questionnaire-Dutch Language Version. Man Ther 2012; 17(4): 330-5.

72. EuroQol Group. EuroQol--a new facility for the measurement of health-related quality of life. Health Policy 1990; 16(3): 199-208.

73. Dowson HM, Ballard K, Gage H, Jackson D, Williams P, Rockall TA. Quality of life in the first 6 weeks following laparoscopic and open colorectal surgery. Value Health 2013; 16(2): 367-72.

74. Schwenkglenks M, Matter-Walstra K. Is the EQ-5D suitable for use in oncology? An overview of the literature and recent developments. Expert Rev Pharmacoecon Outcomes Res 2016; 16(2): 207-19.

75. Clavien PA, Barkun J, de Oliveira ML, et al. The Clavien-Dindo classification of surgical complications: five-year experience. Ann Surg 2009; 250(2): 187-96.

76. Dindo D, Demartines N, Clavien PA. Classification of surgical complications: a new proposal with evaluation in a cohort of 6336 patients and results of a survey. Ann Surg 2004; 240(2): 205-13.

77. Daabiss M. American Society of Anaesthesiologists physical status classification. Indian J Anaesth 2011; 55(2): 111-5.

78. Grossmann I, Klaase JM, Avenarius JK, de Hingh IH, Mastboom WJ, Wiggers T. The strengths and limitations of routine staging before treatment with abdominal CT in colorectal cancer. BMC Cancer 2011; 11: 433. 
79. Dupont WD, Plummer WD,Jr. Power and sample size calculations for studies involving linear regression. Control Clin Trials 1998; 19(6): 589-601.

80. Dupont WD, Plummer WD,Jr. Power and sample size calculations. A review and computer program. Control Clin Trials 1990; 11(2): 116-28.

81. O'Brien PC, Fleming TR. A multiple testing procedure for clinical trials. Biometrics 1979; 35(3): 549-56.

82. Health Council of the Netherlands. Prevention in the elderly: Focus on functioning in daily life. The Hague: Health Council of the Netherlands, 2009; publication no. 2009/07. Available at https://www.gezondheidsraad.nl/sites/default/files/200907r.pdf. Accessed March, 10, 2017.

83. Heerkens YF, de Weerd M, Huber M, et al. Reconsideration of the scheme of the international classification of functioning, disability and health: incentives from the Netherlands for a global debate. Disabil Rehabil 2017: 1-9.

84. Weston M, Weston KL, Prentis JM, Snowden CP. High-intensity interval training (HIT) for effective and time-efficient pre-surgical exercise interventions. Perioper Med (Lond) 2016; 5: 2,015-0026-8. eCollection 2016.

85. Bruns ERJ, Argillander TE, Van Den Heuvel B, et al. Oral Nutrition as a Form of Preoperative Enhancement in Patients Undergoing Surgery for Colorectal Cancer: A Systematic Review. Surg Infect (Larchmt) 2018; 19(1): 1-10.

86. Looijaard SM, Slee-Valentijn MS, Otten RH, Maier AB. Physical and Nutritional Prehabilitation in Older Patients With Colorectal Carcinoma: A Systematic Review. J Geriatr Phys Ther 2018; 41(4): 236-244. 


\title{
Appendix
}

\section{Appendix 6.I. Patient Consent Form}

\author{
Medical scientific research

\section{Registration of data and/or prehabilitation in patients with colorectal cancer or dysplasia undergoing elective colorectal resection}

I was asked to give informed consent to participate in the above mentioned medicalscientific study.

Name:

Date of birth:

- I have read the patient information form. I fully understand the information and I was able to ask questions. My questions have been answered to my satisfaction.

- I know that this research will not contribute to the detection or treatment of my disease.

- I had enough time to decide whether to participate.

- I know that participation is voluntary.

- I know that I may decide at any time not to participate after all or to withdraw from the study. I do not need to give a reason for this. My treatment will not be different.

- I give permission for my GP and treating specialists (if applicable) to be informed about my participation in this study.

- I know that some people can access my data. These people are listed in this information sheet.

- I consent to my data being used in the way and for the purpose stated in the information sheet.

- I consent to my data being stored at the research location for another 15 years after this study.

I want to participate in this study.

Name of study participant:

Signature:

Date: __ $/ \ldots / \ldots$ 
I hereby declare that I have fully informed this study participant about this study.

If information comes to light during the course of the study that could affect the study participant's consent, I will inform him/her of this in a timely fashion.

Name of investigator (or his/her representative):

Signature:

Date: __- / _- / _

The study participant will receive the full information sheet, together with a copy of the signed consent form. 



\section{Abstract}

Background: Patients with a low preoperative aerobic fitness undergoing colorectal surgery have an increased risk of postoperative complications. It remains, however, to be demonstrated whether prehabilitation in these patients reduces postoperative complications. The objective of this study is to assess the effects of a 3-week communitybased exercise program on 30-day postoperative complications in high-risk patients scheduled for elective colorectal resection for (pre)malignancy.

Methods: This two-center, prospective, single-blinded randomized clinical trial was carried out in two large teaching hospitals in the Netherlands. Patients ( $\geq 60$ years) with colorectal (pre)malignancy scheduled for elective colorectal resection and with a score $\leq 7$ metabolic equivalents on the veterans-specific activity questionnaire were randomly assigned to the prehabilitation group or the usual care group by using block-stratified randomization. An oxygen uptake at the ventilatory anaerobic threshold $<11 \mathrm{~mL} / \mathrm{kg} / \mathrm{min}$ at the baseline cardiopulmonary exercise test was the final inclusion criterion. Inclusion was based on a power analysis. Patients in the prehabilitation group participated in a personalized 3-week (three sessions per week, nine sessions in total) supervised exercise program given in community physical therapy practices prior to colorectal resection. Patients in the reference group received usual care. The primary outcome was the number of patients with one or more complications within 30 days of surgery, graded according to the Clavien-Dindo classification. Data were analyzed on an intention-to-treat basis.

Results: Between February 2014 and December 2018, 57 patients ( 30 males and 27 females; mean age 73.6 years (standard deviation 6.1 ), range 61 to 88 years) were randomized to either prehabilitation ( $n=28$ ) or usual care $(n=29)$. The rate of postoperative complications was lower in the prehabilitation group ( $n=12,42.9 \%)$ than in the usual care group ( $n=21$, $72.4 \%$, relative risk $0.59,95 \%$ confidence interval 0.37 to $0.96, p=0.024$ ).

Conclusion: Exercise prehabilitation reduced postoperative complications in highrisk patients scheduled to undergo elective colon resection for (pre)malignancy. Prehabilitation should be considered as usual care in high-risk patients scheduled for elective colon, and probably also rectal, surgery. 


\section{Background}

Colorectal cancer is the third most common cancer worldwide, with an estimated 1.8 million new cases diagnosed and 881,000 deaths associated with the disease in $2018 .{ }^{1}$ Surgical resection of the tumor remains the cornerstone of curative treatment. ${ }^{2}$ However, approximately one-third of patients who undergo colorectal resection experience postoperative complications, ${ }^{3,4}$ which can delay recovery, prolong hospitalization, cause unplanned hospital readmission and chronic illness, and severely impair short- and longterm physical functioning and health-related quality of life. ${ }^{5}$ Reducing complications would considerably reduce the patient burden and costs. ${ }^{3}$ More than $65 \%$ of patients with colorectal cancer are older than 65 years. ${ }^{6}$ In the elderly, the physiological function and reserves of multiple organ systems gradually decline, which affects their tolerance to surgery, ${ }^{7,8}$ with patients with a low preoperative aerobic fitness (oxygen uptake $\left(\mathrm{VO}_{2}\right)$ at the ventilatory anaerobic threshold (VAT) $<11 \mathrm{~mL} / \mathrm{kg} / \mathrm{min}$ ) being particularly at increased risk of postoperative complications. ${ }^{9,10}$ These high-risk, less physically fit patients might therefore benefit from preoperative exercise training (prehabilitation) to optimize their aerobic fitness in order to reduce their risk of morbidity and to facilitate a prompt recovery of physical functioning. ${ }^{11,12}$

To date, studies have provided inconclusive evidence that prehabilitation reduces postoperative complications, as most studies tended to be underpowered, heterogeneous, and biased towards patients with a low risk of postoperative complications., ${ }^{5,12-15} \mathrm{~A}$ recent systematic review of Thomas and colleagues ${ }^{12}$ found that prehabilitation before major intra-abdominal cancer surgery ${ }^{16,17}$ improved postoperative outcomes. They concluded that future prehabilitation research should focus more on the adequate selection of high-risk surgical patients and provide personalized, and probably multimodal, (partly) supervised prehabilitation at home or in a community-based setting with the objective monitoring of a patient's progress. ${ }^{12} \mathrm{Here}$, we describe a randomized clinical trial to study the effects of a 3-week community-based exercise program on 30-day postoperative complications in high-risk patients $\left(\mathrm{VO}_{2}\right.$ at the $\left.\mathrm{VAT}<11 \mathrm{~mL} / \mathrm{kg} / \mathrm{min}\right)$ scheduled for elective colorectal resection for (pre)malignancy.

\section{Methods}

\section{Study design and participants}

This prospective, single-blinded, randomized clinical trial was carried out at two large Dutch teaching hospitals (Medisch Spectrum Twente in Enschede and Ziekenhuisgroep Twente in Almelo). Trial methodology and experimental intervention were designed conform state-of-the-art recommendations (using the Cochrane risk of bias tool and 
CONTENT scale, respectively), as well as by using a clinical decision rule to select the right (high-risk) patients for prehabilitation; ${ }^{18}$ this way, methodological risk of bias is minimized ${ }^{19}$ and therapeutic validity is ensured. ${ }^{20}$ The trial started in February 2014 and inclusion was completed in December 2018. Patients with colorectal cancer or premalignant colorectal lesions (polyps with grade I-III dysplasia that could not be removed endoscopically) scheduled for elective colorectal resection were recruited. Eligible patients were $\geq 60$ years, had a life expectancy $>6$ months as estimated by the surgeon, had a metabolic equivalent of task (MET) score $\leq 7$ on the veterans-specific activity questionnaire (VSAQ), were willing to perform community-based prehabilitation at a physical therapy practice in the catchment area of both hospitals, and were able to perform a progressive cardiopulmonary exercise test (CPET). The VSAQ was used to preselect those patients with a potentially low preoperative aerobic fitness. ${ }^{21}$ We obtained written informed consent from all patients who met the inclusion criteria and were willing to participate. For definite inclusion, patients also had to have a low preoperative aerobic fitness (high risk for postoperative complications) at the baseline CPET, defined as an $\mathrm{VO}_{2}$ at the VAT $<11 \mathrm{~mL} / \mathrm{kg} / \mathrm{min}$. Patients in the prehabilitation group and in the usual care group were all treated within an enhanced recovery pathway according to the enhanced recovery after surgery (ERAS) protocol.22 The study was approved by the local medical ethics committee Twente in Enschede and by the institutional review boards of Ziekenhuisgroep Twente (ZGT), the Netherlands (registration number P13-18), and was registered in the Netherlands Trial Registry (NTR4032). The trial followed the Consolidated Standards of Reporting Trials (CONSORT) reporting guideline ${ }^{23}$ and was conducted according with the ethical standards of the Helsinki Declaration of 1975. A complete overview of the study protocol has been published. ${ }^{24}$

\section{Randomization}

All patients were identified at multidisciplinary oncology meetings and were enrolled at the outpatient clinic by the surgeon via the following procedure: patients with a VSAQ score $\leq 7$ METs were invited to participate in the study and, after providing their informed consent, they were randomly assigned to the prehabilitation group or the usual care group by block-stratified randomization. ${ }^{25} \mathrm{After}$ randomization, all participants performed a CPET to verify study eligibility $\left(\mathrm{VO}_{2}\right.$ at the VAT $\left.<11 \mathrm{~mL} / \mathrm{kg} / \mathrm{min}\right)$. A research nurse who did not help recruit patients or perform data analyses performed the randomization by using sealed opaque envelopes, based on computer generated randomization lists. Randomization was stratified by disease and treatment type: 1) patients with colon cancer or premalignancy, 2) patients with rectal cancer who would receive 1 week of neoadjuvant radiotherapy, and 3) patients with rectal cancer who would receive 5 weeks of neoadjuvant chemoradiotherapy. Participants were unaware of the study hypothesis: they knew they were allocated to one of two groups to assess the effects of physical fitness on postoperative outcome, but they did not know that one program was developed as 
an intervention and the other as a control. Subsequently, patients in the prehabilitation group were informed about all aspects of the study. No advice about preoperative exercise training was offered to patients in the usual care group in order to avoid the risk that these patients initiated preoperative exercise interventions themselves, and they were planned for surgery at the earliest convenience (with explicit permission from the medical ethics committee). Participants and care providers were not masked to randomization, because of the type of intervention. Data collection and analysis were performed blinded to the group allocation of the patients.

\section{Intervention}

Ideally, the baseline assessment of the patients was performed within a week of their first visit at the outpatient clinic. Patients in the prehabilitation group were reassessed after the preoperative exercise program a few days before the surgical procedure. Patients in the usual care group underwent surgery as soon as possible (normally within 2-3 weeks). Usual care consisted of nutritional counseling and advice on smoking cessation.

Patients in the prehabilitation group participated in a personalized 3-week (3 sessions per week, 9 sessions in total) supervised exercise program prior to colorectal resection. These exercises were executed and/or supervised by a group of trained physical therapists, under the guidance of $\mathrm{HK}$ and $\mathrm{PW}$, in community physical therapy practices in the catchment area of both hospitals. Patients with colon (pre)malignancy participated in the prehabilitation program during the period between the decision to undergo surgery and the actual procedure, whereas patients with rectal cancer due to receive neoadjuvant therapy completed the prehabilitation program prior to radiotherapy (in case of neoadjuvant radiotherapy, 5x5 Gy) or in the 12-week period after completing neoadjuvant chemoradiotherapy (in week 10-12). Each 60-minute training session consisted of moderate-to-high intensity interval training on a cycle ergometer (TechnoGym, Bike Med, Gambettola, Italy) to improve aerobic fitness (40 minutes), and resistance training to improve peripheral muscle strength (20 minutes). Detailed information about the intervention can be found in the Appendix 7.1.

\section{Outcomes}

The primary endpoint was the number of patients with one or more complications within 30 days of surgery. Complications were divided into surgical and non-surgical complications and graded according to the Clavien-Dindo classification..$^{26,27}$ Intermediate outcome measures were change in preoperative aerobic fitness (the $\mathrm{VO}_{2}$ at the VAT) in the prehabilitation group, length of hospital stay, and unplanned readmissions within 30 and 90 days after surgery.

At baseline, participants in both groups underwent a progressive CPET (Oxycon Pro, 
Jaeger, Hoechberg, Germany in both hospitals) on a calibrated electronically braked cycle ergometer (Ergoline, Ergoselect 100, Bitz, Germany at Medisch Spectrum Twente and Lode Excalibur Sport, Lode BV, Groningen, the Netherlands at Ziekenhuisgroep Twente). Throughout the CPET, breath-by-breath measurement of $\mathrm{VO}_{2}$, carbon dioxide production, respiratory flow, and volume parameters, and twelve-lead electrocardiography was performed to verify study eligibility, to assess baseline aerobic fitness (e.g., the $\mathrm{VO}_{2}$ at the VAT), to check for potential contraindications, and to personalize the interval training program. ${ }^{24}$ Additional information concerning the interpretation of the CPET data can be found in Appendix 7.2.

Muscle strength assessment included handgrip strength and quadriceps strength. ${ }^{24}$ In addition to clinical history and physical examination, the following descriptive tests were also included (see for detailed information our study protocol):24 timed-up-and-go test, short nutritional assessment questionnaire (SNAQ), and the Groningen frailty indicator. Only the prehabilitation group performed the second CPET after the 3-week exercise program.

\section{Sample size calculation}

On the basis of morbidity rates in colorectal patients reported in the Dutch ColoRectal Audit $(30 \%)^{2}$ and the literature (21\% in non-frail patients, $40 \%$ in pre-frail patients, and $58 \%$ in frail patients), ${ }^{28}$ we hypothesized that the complication rate in high-risk patients $\left(\mathrm{VO}_{2}\right.$ at the $\left.\mathrm{VAT}<11 \mathrm{~mL} / \mathrm{kg} / \mathrm{min}\right)$ undergoing colorectal surgery without prehabilitation would be around $50 \%$, and after prehabilitation about $20 \%$. To detect a statistically significant difference between groups, we calculated that 43 patients in each group would be required ( $\alpha$ of 0.0492 , due to one interim analysis, $B$ of $80 \%$, taking a $10 \%$ drop out rate into account). Our interim analysis (date July 11, 2017), with a stopping rule according to O'Brien-Fleming that aimed to test if the study should be stopped due to superiority or futility, ${ }^{29}$ showed a significantly lower incidence of complications (33\% versus $73 \% ; p=0.0096)$ in patients in the prehabilitation group $(n=21)$ than in patients in the usual care group ( $n=22)$. These data were used to recalculate our sample size, with explicit permission from the Medical Ethics Committee. Now, 27 patients in each group were required to detect statistically significant differences between groups ( $\alpha$ of $0.0492, B$ of $80 \%$, taking a $10 \%$ dropout rate into account). See Appendix 7.3 for more information.

\section{Statistical analysis}

Data (coded on study code) were analyzed with the Statistical Package for the Social Sciences for Windows (version 23.0; IBM, SPSS Inc., Chicago, IL, USA). For continuous variables, independent samples t-tests or Mann-Whitney $U$ tests, as appropriate, were performed to analyze differences between the two groups. For categorical variables this was done with chi-squared tests or Fisher's exact tests, as appropriate. Variables were 
tested for their association with postoperative complications $(p<0.05)$, using the t-test, Mann Whitney $U$ test, Fisher's exact test or chi-squared test, as appropriate. A repeated measurements analysis (mixed models) was performed to assess changes over time in continuous variables. A multivariable logistic regression analysis was performed to identify independent predictors of a postoperative complication, using a forward stepwise procedure ( $p$ in $0.05, p$ out 0.10 ). If there was multicollinearity between variables, the variable that produced the best model fit (based on the -2 log likelihood) was included in the model. A new logistic regression model was made (method: enter) incorporating the selected significant variables, to utilize the maximum number of observations. Receiver operator curve (ROC) analysis was used to assess the independent ability of predictive variables to discriminate between patients with and without a postoperative complication. All tests were performed on both the intention-to-treat population and the per-protocol population. Because of the interim analysis, the final $p$-value considered to be statistically significant was reduced to $<0.0492$ (2-sided).

\section{Results}

Between February 1, 2014 and the final inclusion at December 31, 2018, 682 potential participants were assessed for study participation. Of these, 132 met the inclusion criteria, of which 74 (56\%) gave their consent to participate (Figure 1). Of these 74 patients, 17 were excluded from the analysis ( $n=12$ had a VO $\mathrm{V}_{2}$ at the VAT $>11 \mathrm{~mL} / \mathrm{kg} / \mathrm{min}$ and $n=5$ did not undergo surgery). Of the remaining 57 patients (30 males, 27 females; mean age 73.6 years (standard deviation (SD) 6.1), range 61 to 88 years), 28 were randomized to the prehabilitation group and 29 to the usual care group. Of the 57 patients, 3 had rectal cancer, of which 2 received neoadjuvant chemoradiation.

Baseline characteristics of study participants are presented in Table 1 and were largely similar between both groups, except for time between inclusion and surgery $(p<0.001)$.

Median time between the first visit at the outpatient clinic and the baseline CPET was 4 days (interquartile range (IQR) 3-6). Mean time between the second CPET and surgery for patients in the prehabilitation group was 2.4 days (SD 1.6). In the prehabilitation group, $\mathrm{VO}_{2}$ at the VAT had improved by $10.1 \%(+0.97 \mathrm{~mL} / \mathrm{kg} / \mathrm{min}(n=24), 95 \%$ confidence interval (Cl) 0.3 to $1.6 ; p=0.006)$ and $\mathrm{VO}_{2 \text { eak }}$ had improved by $8.8 \%(+1.3 \mathrm{~mL} / \mathrm{kg} / \mathrm{min}(n=16), 95 \% \mathrm{Cl}$ -0.006 to $2.6 ; p=0.051$ ) after the 3-week program; see Figure 2 . Ten (36\%) patients showed no or minimal improvement $\left(<+0.5 \mathrm{~mL} / \mathrm{kg} / \mathrm{min} \mathrm{VO}_{2}\right.$ at the VAT) at the second CPET. Quadriceps strength had increased on average by $2.1 \%$ ( +6.6 Newton ( $n=23), 95 \% \mathrm{Cl}-21.4$ to $8.2 ; p=0.37$ ). None of the patients reported adverse events during the exercise program and none were observed by the physical therapists. Patients attended 8.1 (SD 2.4) of the nine supervised exercise training sessions (90\%). 


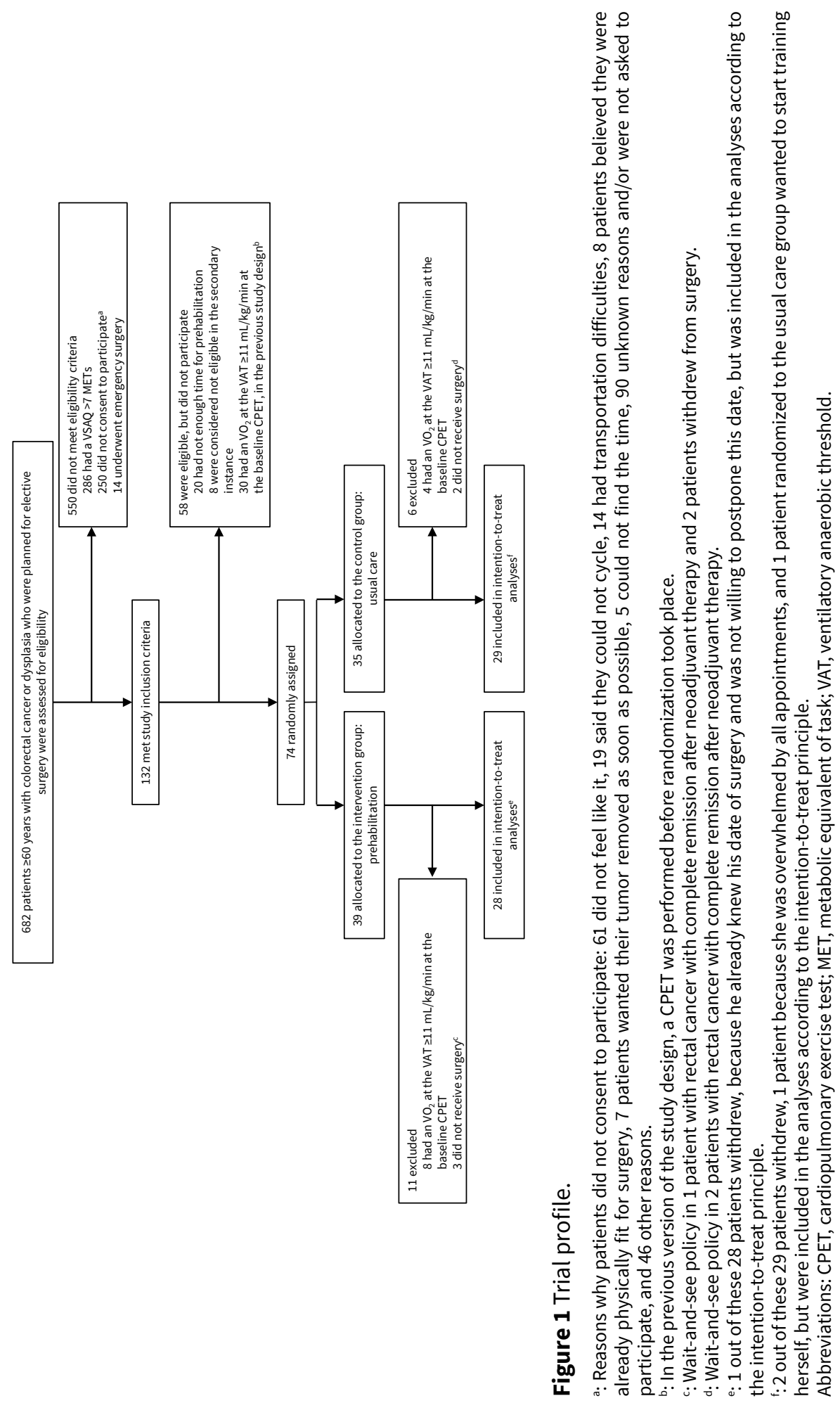


Table 1 Baseline characteristics.

\begin{tabular}{|c|c|c|}
\hline Parameter & $\begin{array}{l}\text { Prehabilitation group } \\
(n=28)\end{array}$ & $\begin{array}{l}\text { Usual care group } \\
\qquad(n=29)\end{array}$ \\
\hline Age (years) & $74(7)$ & $73(6)$ \\
\hline Sex ratio $(M: F)$ & $16(57 \%): 12(43 \%)$ & $14(48 \%): 15(52 \%)$ \\
\hline Body mass index $\left(\mathrm{kg} / \mathrm{m}^{2}\right)$ & $29.8(4.1)$ & $30.5(4.9)$ \\
\hline Smokinga & $1(4 \%)$ & $6(21 \%)$ \\
\hline \multicolumn{3}{|l|}{ Age-adjusted Charlson comorbidity index ${ }^{b}$} \\
\hline $2-3$ & $12(43 \%)$ & $8(28 \%)$ \\
\hline $4-5$ & $12(43 \%)$ & $14(48 \%)$ \\
\hline $6+$ & $4(14 \%)$ & $7(24 \%)$ \\
\hline \multicolumn{3}{|l|}{ ASA score } \\
\hline I & $0(0 \%)$ & $1(3 \%)$ \\
\hline II & $21(75 \%)$ & $24(83 \%)$ \\
\hline III & $7(25 \%)$ & $4(14 \%)$ \\
\hline $\mathrm{VO}_{2}$ at the VAT $(\mathrm{mL} / \mathrm{kg} / \mathrm{min})^{\mathrm{c}}$ & $9.6(1.2)$ & $9.1(1.1)$ \\
\hline $\mathrm{VO}_{2 \text { peak }}(\mathrm{mL} / \mathrm{kg} / \mathrm{min})^{\mathrm{d}}$ & $14.7(3.1)$ & $14.4(2.8)$ \\
\hline Hemoglobin level (mmol/L)e & $7.9(1.1)$ & $7.8(1.2)$ \\
\hline Timed up-and-go test $(\mathrm{s})^{\mathrm{f}}$ & $9.7(2.2)$ & $9.6(4.0)$ \\
\hline MET score on VSAQ & $4.6(1.4)$ & $4.3(1.3)$ \\
\hline SNAQ scoreg & $0.4(1.1)$ & $0.4(0.8)$ \\
\hline GFI score & $3(2)$ & $3(3)$ \\
\hline \multicolumn{3}{|l|}{ Surgical procedure } \\
\hline Right hemicolectomy & $15(54 \%)$ & $12(41 \%)$ \\
\hline Transverse hemicolectomy & $1(4 \%)$ & $1(3 \%)$ \\
\hline Left hemicolectomy & $2(7 \%)$ & $3(10 \%)$ \\
\hline Sigmoid colectomy & $9(32 \%)$ & $9(31 \%)$ \\
\hline APR & $1(4 \%)$ & $0(0 \%)$ \\
\hline LAR & $0(0 \%)$ & $1(3 \%)$ \\
\hline Subtotal colectomy & $0(0 \%)$ & $1(3 \%)$ \\
\hline Other & $0(0 \%)$ & $2(7 \%)$ \\
\hline \multicolumn{3}{|l|}{ Type of surgery } \\
\hline Open & $0(0 \%)$ & $2(7 \%)$ \\
\hline Laparoscopic & $23(82 \%)$ & $21(72 \%)$ \\
\hline Conversion to open & $5(18 \%)$ & $6(21 \%)$ \\
\hline Time between inclusion and surgery (days) & $34.6(28.8)$ & $19.0(10.2)$ \\
\hline
\end{tabular}

Data are number of patients (\%), median (IQR) or mean (SD).

a: 4 missing in the prehabilitation group, so in this case $n=24$.

b: Each decade of age over 40 adds 1 point to risk (50-59 years, 1 point; 60-69 years, 2 points; $70-79$ years, 3 points) and these points for age are added to the score from the Charlson comorbidity index (e.g., 0, 1, 2, 3, et cetera). ${ }^{41}$ c: 1 missing in the usual care group, so in this case $n=28$.

$\mathrm{d}: 10$ patients (36\%) in the prehabilitation group and 11 patients $(38 \%)$ in the usual care group did not meet the criteria for a valid maximal effort at the baseline CPET, and 1 patient in the usual care group did not performed a CPET, so in this case $n=18$ and $n=17$, respectively.

e: 2 missing in the prehabilitation group, so in this case $n=26$. Hemoglobin level was assessed within three months before surgery. 
f: 1 missing in the prehabilitation group and 7 missing in the usual care group, so in this case $n=27$ and $n=22$, respectively.

g: 1 missing in the prehabilitation group and 3 missing in the usual care group, so in this case $n=27$ and $n=26$, respectively.

h: 2 missing in the prehabilitation group and 4 missing in the usual care group, so in this case $n=26$ and $n=25$, respectively.

Abbreviations: APR, abdominal perineal resection; ASA, American Society of Anesthesiologists; GFI, Groningen frailty indicator; LAR, low anterior resection; MET, metabolic equivalent of task; SNAQ, short nutritional assessment questionnaire; VAT, ventilatory anaerobic threshold; $\mathrm{VO}_{2}$, oxygen uptake; $\mathrm{VO}_{2 \text { peak }}$, oxygen uptake at peak exercise; VSAQ, veterans-specific activity questionnaire.
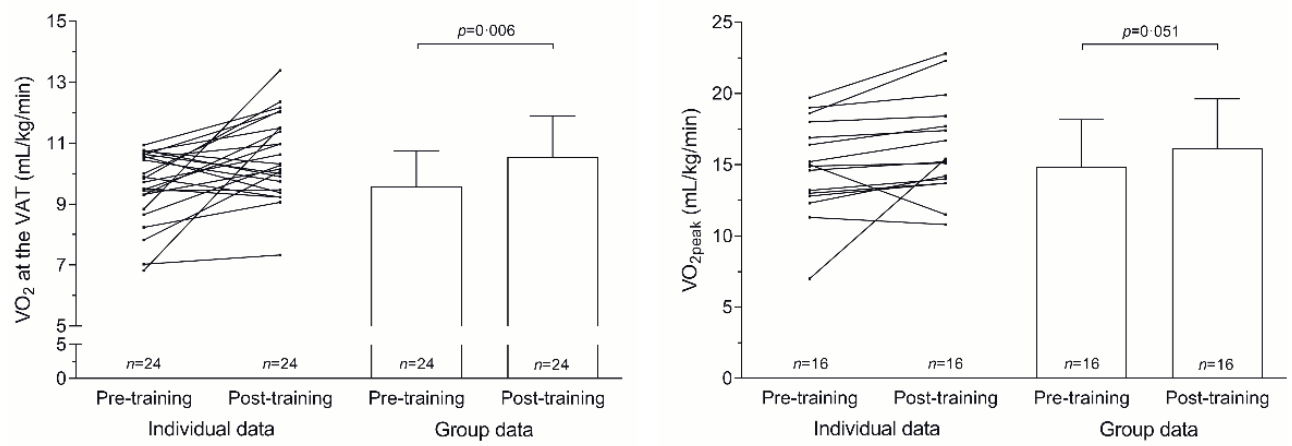

Figure 2 Pre- and post-training $\mathrm{VO}_{2}$ at the $\mathrm{VAT}$ and $\mathrm{VO}_{2 \text { eak }}$ in patients randomized for prehabilitation.

$n=24$ for $\mathrm{VO}_{2}$ at the VAT, as 4 patients did not perform a post-training CPET.

$n=16$ for $\mathrm{VO}_{2 \text { peak }}$, as 4 patients did not perform a post-training CPET and 8 patients did not perform a maximal effort on both CPETs.

Abbreviations: CPET, cardiopulmonary exercise test; VAT, ventilatory anaerobic threshold; $\mathrm{VO}_{2}$, oxygen uptake; $\mathrm{VO}_{2 \text { peak }}$, oxygen uptake at peak exercise.

The overall complication rate in the full cohort was 58\%. One patient randomized to prehabilitation died as a result of complications arising from a leaking anastomosis. However, this patient had withdrawn from the study directly after randomization because his surgery had been planned during the study period and he did not want to postpone surgery. Both patients with rectal cancer who received neoadjuvant chemoradiation and who were randomized to the prehabilitation group had grade 2 complications. The third patient with rectal cancer, who did not receive neoadjuvant therapy, was randomized to the usual care group and also had a postoperative complication (grade 1).

The complication rate in patients in the prehabilitation group ( $n=12,42.9 \%$ ) was significantly lower than that of patients in the usual care group $(n=21,72.4 \%, p=0.024$, Table 2). There were no differences in the type of complications between the two groups. Analysis showed that prehabilitation had a protective role against overall postoperative complications (relative risk $0.59,95 \% \mathrm{Cl} 0.37$ to 0.96 ). No differences in hospital readmission rates were found between the two groups $(n=4,14.3 \%$ in the prehabilitation 
group vs. $n=5,17.2 \%$ in the usual care group; $p>0.99$ ).

In the univariable analysis of all baseline characteristics (see Table 1), hemoglobin level $(p=0.002)$, age-adjusted Charlson score $(p=0.024)$, and SNAQ score $(p=0.084)$ were associated with postoperative complications. In the final multivariable model including hemoglobin level and prehabilitation, a higher preoperative hemoglobin level (OR 0.37, $95 \% \mathrm{Cl} 0.18$ to $0.75 ; p=0.006$ ) and prehabilitation (OR $0.21,95 \% \mathrm{Cl} 0.06$ to $0.79 ; p=0.021$ ) were individually associated with a decreased 30 -day risk of postoperative complications. Patients with a $1.0 \mathrm{mmol} / \mathrm{L}$ higher hemoglobin level were three times less likely to have a postoperative complication than patients with a lower hemoglobin level. ROC analysis for predicting patients with a postoperative complication based on the hemoglobin level and prehabilitation gave an AUC of 0.77 ( $95 \% \mathrm{Cl} 0.65$ to $0.89 ; p=0.001$; Figure 3 ).

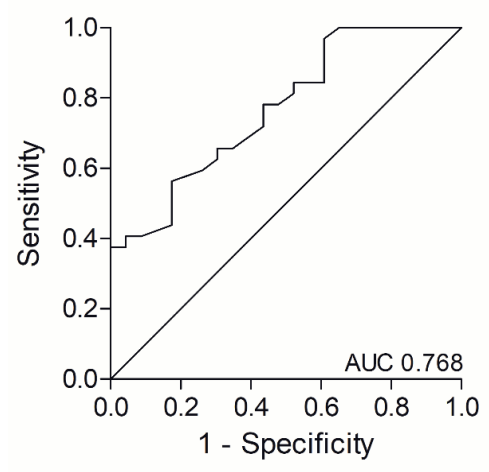

Figure $3 \mathrm{ROC}$ analysis for predicting patients with a postoperative complication, based on the preoperative hemoglobin level and prehabilitation.

Abbreviations: AUC, area under the curve; ROC, receiver operator curve. 


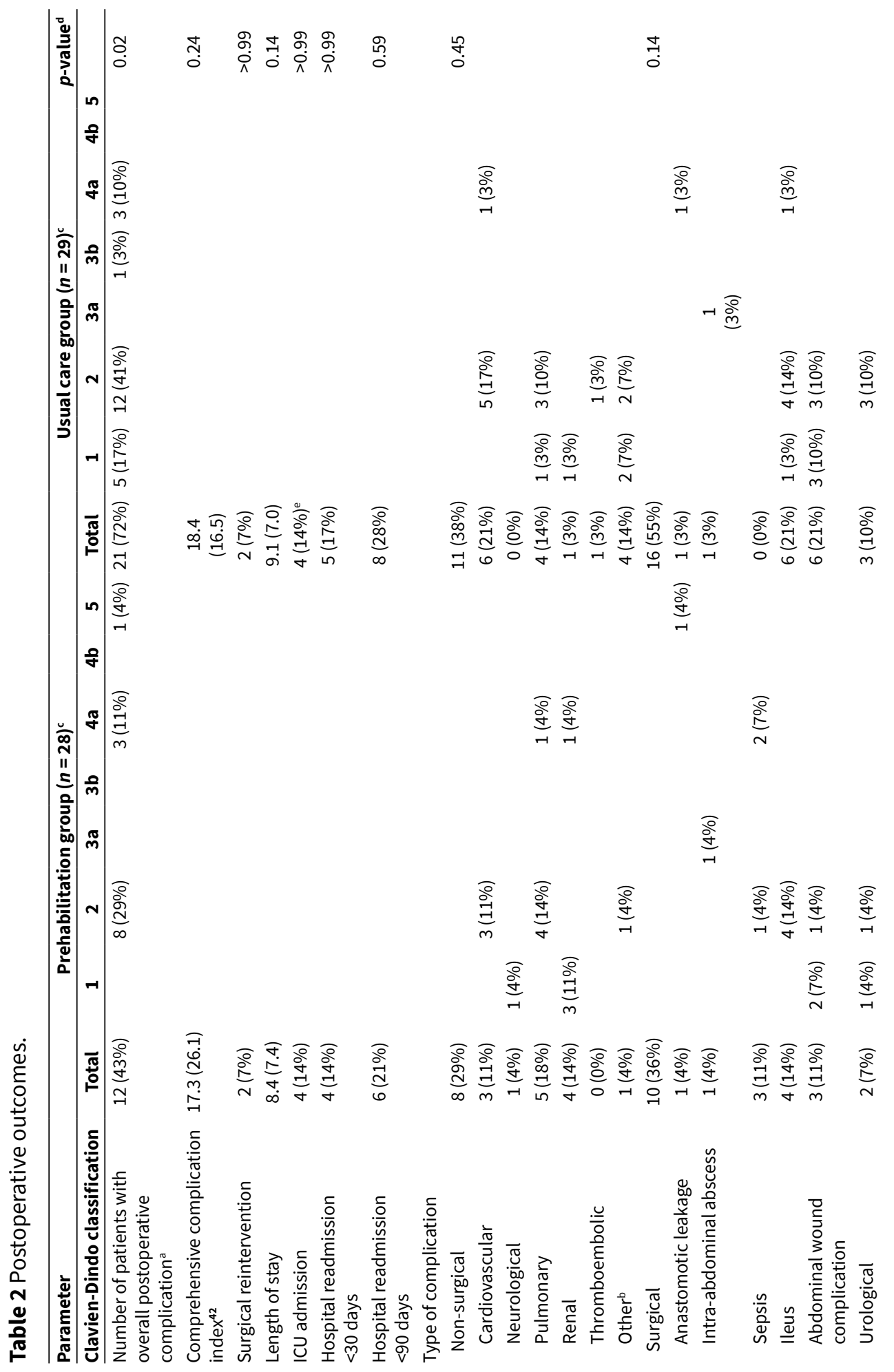




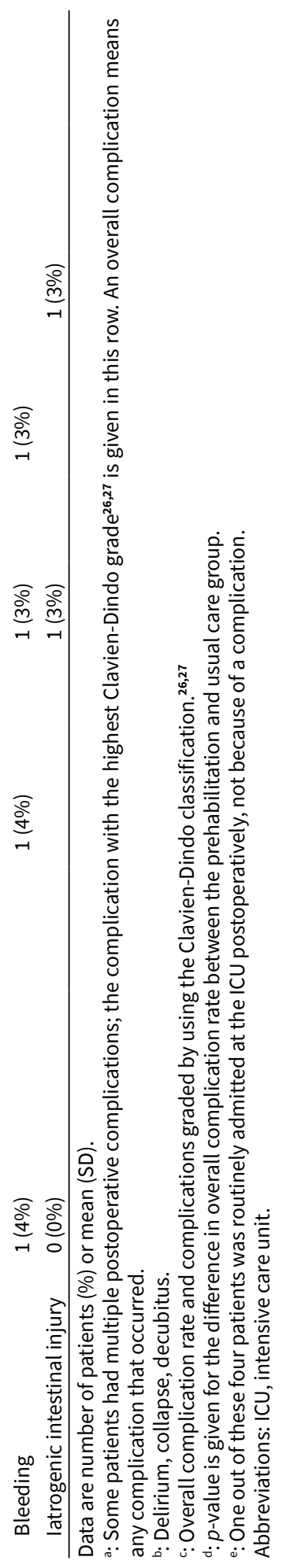




\section{Discussion}

To the best of our knowledge, this is the first state-of-the-art randomized clinical trial to evaluate the effects of a 3-week community-based and personalized prehabilitation program for patients scheduled for surgery for colorectal (pre)malignancy who were considered to be at high risk of postoperative complications, as established with a preoperative clinical decision rule. Within a relatively short period of 3 weeks, prehabilitation improved aerobic fitness by approximately $10 \%$ and patients subsequently experienced an almost 50\% decrease in the incidence of postoperative complications. The program thus appears to make patients more resistant to the potentially negative consequences of some or all ingredients and perceptions of the perioperative trajectory, so that they experience fewer postoperative complications.

With regard to the overall complication rate in this study (58\%) one has to bear in mind that the population under study is that of a high-risk population, known to demonstrate incidences of about $50 \%$ or even more. For instance, comparable studies like that of West et al. ${ }^{9}$ showed an overall morbidity rate after major colorectal surgery of $65 \%$ in patients with a low preoperative aerobic fitness (oxygen consumption at the ventilatory anaerobic threshold $<11 \mathrm{~mL} / \mathrm{kg} / \mathrm{min}$ ). Moreover, Barberan-Garcia et al. ${ }^{17}$ found an overall incidence of complications of $46 \%$ in high-risk patients (age $>70$ years, ASA score III/IV, and Duke activity status index score $\leq 46$ ) undergoing major abdominal surgery. Although prehabilitation cannot prevent all complications, the impact of a postoperative complication might also be reduced in patients with a higher physical fitness, ${ }^{12,30}$ as previously indicated in coronary artery bypass graft surgery and pancreatic surgery. ${ }^{31,32}$

Barberan-Garcia and colleagues ${ }^{17}$ reported that prehabilitation prior to elective major abdominal surgery significantly improved preoperative aerobic fitness and significantly reduced the number of high-risk patients with postoperative complications (RR $0.5,95 \% \mathrm{Cl}$ 0.3 to 0.8 ), as compared to usual care. However, they selected high-risk patients based on age, ASA score, and Duke activity status index score and not on formal (cardiopulmonary) exercise testing. Moreover, Carli et al. ${ }^{15}$ recently showed that prehabilitation in (pre)frail patients (based on the Fried frailty index) ${ }^{33}$ who underwent colorectal cancer resection did not reduce postoperative complications. Patients with a low preoperative aerobic fitness would be expected to benefit the most from prehabilitation. ${ }^{11,12}$ Therefore, after making a preselection of potentially high-risk patients (e.g., with the VSAQ), further selection of high-risk patients on the basis of CPET findings might be the risk screening strategy of choice. In our study, patients with a lower preoperative hemoglobin level were at greater risk of postoperative complications. This suggests that the preoperative hemoglobin level should also be taken into consideration when stratifying patients by risk. Pre-optimization of iron deficiency anaemia by means of iron supplements in surgical patients undergoing 
major abdominal surgery increases preoperative hemoglobin concentrations, decreases the need for transfusion, ${ }^{34}$ and results in a shorter hospital stay. ${ }^{35}$ Use of erythropoietin is not recommended, because it potentially increases the risk of thrombosis, mortality, ${ }^{36}$ and tumor growth. ${ }^{37}$

Our study followed current recommendations regarding the use of a clinical decision rule to minimize the risk of bias and to ensure therapeutic validity. ${ }^{18-20}$ Nevertheless, our prehabilitation approach could be improved. First, patient inclusion was challenging, and we had a low participation rate (56\%) among patients who met (preliminary) inclusion criteria. The main reasons given for non-participation were no inclination to participate, inability to perform cycling exercises, considered self to be physically fit, or desire for surgery as soon as possible (Figure 1). This suggests that it is important to present preoperative risk stratification and prehabilitation as an integral part of the perioperative care package for high-risk patients, in which patients, their relatives, and their (in)formal caregivers should be adequately informed about the importance of physical activity and physical fitness before and after surgery in relation to surgical outcomes. Moreover, the context of delivering prehabilitation should be well-considered, as patients prefer homebased preoperative physical exercise training, supervised once a week, ${ }^{38}$ ideally by a dedicated and competent community physical therapist. Second, our prehabilitation program was a unimodal program, focusing on exercise, whereas a recent systematic review by Thomas and colleagues ${ }^{12}$ reported that multimodal programs including exercise training, nutritional support, psychological support, and the interaction between these components might be most effective and should be considered in further research. However, definitive clinical evidence of the feasibility and cost-effectiveness of multimodal prehabilitation is currently very limited, and should be investigated further. ${ }^{12,39}$ Third, we did not objectively assess whether and how a patient's physical fitness (aerobic and muscular fitness) improved during prehabilitation. Instead, we adjusted the program each week in a standardized way. When measured objectively and on multiple occasions, training intensity can be adjusted according to the improvement in physical fitness, to maintain an effective training stimulus. Moreover, frequent monitoring of progress is essential to identify non-responders or non-compliant individuals as soon as possible, because of the short time window available for the intervention before surgery. ${ }^{12}$ Strous and colleagues recently showed that prehabilitation programs can safely be extended because a prolonged treatment delay did not lead to poorer overall or cancer-free survival in patients with primary colorectal cancer who underwent curative surgical treatment. ${ }^{40} \mathrm{In}$ the present study, 10 of the 28 patients in the prehabilitation group were considered post hoc to be non-responders $(n=7)$ or minimal responders $(<+0.5 \mathrm{~mL} / \mathrm{kg} / \mathrm{min}, n=3)$, based on the $\mathrm{VO}_{2}$ at the VAT at the second CPET. Of these 10 patients, 6 had a postoperative complication (60\%), whereas this was $43 \%$ of the total prehabilitation group and $72 \%$ of the usual care group. Ideally, aerobic fitness should improve in all high-risk patients, 
and achieving this will be a challenge for future research. If we had monitored progress in order to adjust the intervention as needed, we might have seen a greater effect of prehabilitation.

The study had some other limitations. Although adequately powered, the patient group was small and selective. Moreover, the ROC analysis was performed in a small cohort and might therefore not be very robust. Since only 3 patients with rectal cancer were included in the study, their findings possibly cannot be generalized to this patient group. Lastly, the decision not to include the 5 patients who were randomized but who ultimately did not undergo surgery in the intention-to-treat analysis is debatable.

In future research, attempts should be made to ensure that all eligible high-risk patients are willing and able to participate in prehabilitation programs. Moreover, as mentioned before, prehabilitation should ideally be performed in high-risk patients selected with a preoperative CPET, and must be multimodal, personalized, (partly) supervised, and homeor community-based, with frequent objective monitoring of progress and subsequent adjustment of training frequency and/or intensity. We suggest using a stepped wedge design, with modern data techniques, to implement and evaluate prehabilitation in reallife care of high-risk patients undergoing colorectal surgery.

In conclusion, our study showed that community-based exercise prehabilitation reduced the risk of postoperative complications in high-risk patients scheduled for elective colon resection for (pre)malignancy. Probably, this is also true for high-risk patients undergoing rectal resection; however, the small number of patients with rectal cancer included in this study makes it impossible to draw conclusions about the effectiveness of prehabilitation in this specific patient group. Prehabilitation should be considered as usual care in highrisk patients scheduled for elective colon, and probably also rectal, surgery. 


\section{References}

1. Bray F, Ferlay J, Soerjomataram I, Siegel RL, Torre LA, Jemal A. Global cancer statistics 2018: GLOBOCAN estimates of incidence and mortality worldwide for 36 cancers in 185 countries. CA Cancer J Clin 2018; 68(6): 394-424.

2. Dutch institute for clinical auditing (DICA) Leiden. DICA jaarrapportage 2018: Dutch ColoRectal Audit (DCRA). Available at: https://dica.nl/jaarrapportage-2018/dcra. Last accessed April 20, 2020.

3. Govaert JA, Fiocco M, van Dijk WA, et al. Costs of complications after colorectal cancer surgery in the Netherlands: Building the business case for hospitals. Eur J Surg Oncol 2015; 41(8): 1059-67.

4. Tevis SE, Kennedy GD. Postoperative Complications: Looking Forward to a Safer Future. Clin Colon Rectal Surg 2016; 29(3): 246-52.

5. Moran J, Guinan E, McCormick P, et al. The ability of prehabilitation to influence postoperative outcome after intra-abdominal operation: A systematic review and meta-analysis. Surgery 2016; 160(5): 1189-201.

6. Integraal Kankercentrum Nederland (IKNL). Cijfers over kanker. February 2, 2019; Available at: https://www.cijfersoverkanker.nl/selecties/dataset_2/img5d2314aa65ca5. Accessed July, 8, 2019.

7. Santa Mina D, Scheede-Bergdahl C, Gillis C, Carli F. Optimization of surgical outcomes with prehabilitation. Appl Physiol Nutr Metab 2015; 40(9): 966-9.

8. Hulzebos EH, van Meeteren NL. Making the elderly fit for surgery. Br J Surg 2016; 103(4): 463.

9. West MA, Asher R, Browning M, et al. Validation of preoperative cardiopulmonary exercise testing-derived variables to predict in-hospital morbidity after major colorectal surgery. $\mathrm{Br} \mathrm{J}$ Surg 2016; 103(6): 744-52.

10. Moran J, Wilson F, Guinan E, McCormick P, Hussey J, Moriarty J. Role of cardiopulmonary exercise testing as a risk-assessment method in patients undergoing intra-abdominal surgery: a systematic review. Br J Anaesth 2016; 116(2): 177-91.

11. Bongers BC, Punt IM, van Meeteren NL. On "Prehabilitation: The emperor's new clothes or a new arena for physical therapists?" Lundberg M, Archer KR, Larsson C, Rydwik E. Phys Ther. 2018;12:127-130. Phys Ther 2019; 99(7): 953-4.

12. Thomas G, Tahir MR, Bongers BC, Kallen VL, Slooter GD, van Meeteren NL. Prehabilitation before major intra-abdominal cancer surgery: A systematic review of randomised controlled trials. Eur J Anaesthesiol 2019; 36(12): 933-45.

13. Bruns ER, van den Heuvel $B$, Buskens $C J$, et al. The effects of physical prehabilitation in elderly patients undergoing colorectal surgery: a systematic review. Colorectal Dis 2016; 18(8): O26777.

14. Hijazi Y, Gondal U, Aziz O. A systematic review of prehabilitation programs in abdominal cancer surgery. Int J Surg 2017; 39: 156-62. 
15. Carli F, Bousquet-Dion G, Awasthi R, et al. Effect of Multimodal Prehabilitation vs Postoperative Rehabilitation on 30-Day Postoperative Complications for Frail Patients Undergoing Resection of Colorectal Cancer: A Randomized Clinical Trial. JAMA Surg 2020; 155(3): 233-242.

16. Dronkers JJ, Lamberts $\mathrm{H}$, Reutelingsperger IM, Naber RH, Dronkers-Landman CM, Veldman $A$, van Meeteren NL. Preoperative therapeutic programme for elderly patients scheduled for elective abdominal oncological surgery: a randomized controlled pilot study. Clin Rehabil 2010; 24(7): 614-22.

17. Barberan-Garcia A, Ubre M, Roca J, et al. Personalized Prehabilitation in High-risk Patients Undergoing Elective Major Abdominal Surgery: A Randomized Blinded Controlled Trial. Ann Surg 2018; 267(1): 50-6.

18. McGinn TG, Guyatt GH, Wyer PC, Naylor CD, Stiell IG, Richardson WS. Users' guides to the medical literature: XXII: how to use articles about clinical decision rules. Evidence-Based Medicine Working Group. JAMA 2000; 284(1): 79-84.

19. Higgins JP, Altman DG, Gotzsche PC, et al. The Cochrane Collaboration's tool for assessing risk of bias in randomised trials. BMJ 2011; 343: d5928.

20. Hoogeboom TJ, Oosting E, Vriezekolk JE, et al. Therapeutic validity and effectiveness of preoperative exercise on functional recovery after joint replacement: a systematic review and meta-analysis. PLoS One 2012; 7(5): e38031.

21. Snowden CP, Prentis JM, Anderson HL, Roberts DR, Randles D, Renton M, Manas DM. Submaximal cardiopulmonary exercise testing predicts complications and hospital length of stay in patients undergoing major elective surgery. Ann Surg 2010; 251(3): 535-41.

22. Gustafsson UO, Scott MJ, Schwenk W, et al. Guidelines for perioperative care in elective colonic surgery: Enhanced Recovery After Surgery (ERAS $\left.\left({ }^{\circledast}\right)\right)$ Society recommendations. World J Surg 2013; 37(2): 259-84.

23. Moher D, Hopewell S, Schulz KF, et al. CONSORT 2010 explanation and elaboration: updated guidelines for reporting parallel group randomised trials. BMJ 2010; 340: c869.

24. Berkel AEM, Bongers BC, van Kamp MS, et al. The effects of prehabilitation versus usual care to reduce postoperative complications in high-risk patients with colorectal cancer or dysplasia scheduled for elective colorectal resection: study protocol of a randomized controlled trial. BMC Gastroenterol 2018; 18(1): 29,018-0754-6.

25. Press WH, Teukolsky S, Vetterling WT, et al. Numerical Recipes in C: the Art of Scientific Computing. Cambridge University Press; 1992:280.

26. Clavien PA, Barkun J, de Oliveira ML, et al. The Clavien-Dindo classification of surgical complications: five-year experience. Ann Surg 2009; 250(2): 187-96.

27. Dindo D, Demartines N, Clavien PA. Classification of surgical complications: a new proposal with evaluation in a cohort of 6336 patients and results of a survey. Ann Surg 2004; 240(2): 205-13.

28. Robinson TN, Wu DS, Pointer L, Dunn CL, Cleveland JC,Jr, Moss M. Simple frailty score predicts postoperative complications across surgical specialties. Am J Surg 2013; 206(4): 544-50. 
29. O'Brien PC, Fleming TR. A multiple testing procedure for clinical trials. Biometrics 1979; 35(3): 549-56.

30. Bongers BC, Dejong CHC, den Dulk M. Enhanced recovery after surgery programmes in older patients undergoing hepatopancreatobiliary surgery: what benefits might prehabilitation have? Eur J Surg Oncol 2021; 47(3 Pt A): 551-559.

31. Hulzebos EH, Helders PJ, Favie NJ, De Bie RA, Brutel de la Riviere A, Van Meeteren NL. Preoperative intensive inspiratory muscle training to prevent postoperative pulmonary complications in high-risk patients undergoing CABG surgery: a randomized clinical trial. JAMA 2006; 296(15): 1851-7.

32. Van Beijsterveld CAFM, Bongers BC, Den Dulk M, Van Kuijk SMJ, Dejong CHC, Van Meeteren NLU. Exploring the relation between preoperative physical functioning and the impact of major complications in patients following pancreatic resection. HPB (Oxford) 2020; 22(5): 71627.

33. Fried LP, Tangen CM, Walston J, et al. Frailty in older adults: evidence for a phenotype. $J$ Gerontol A Biol Sci Med Sci 2001; 56(3): M146-56.

34. Munting KE, Klein AA. Optimisation of preoperative anaemia in patients before elective major surgery - why, who, when and how? Anaesthesia 2019; 74(Suppl 1): 49-57.

35. Froessler B, Palm P, Weber I, Hodyl NA, Singh R, Murphy EM. The Important Role for Intravenous Iron in Perioperative Patient Blood Management in Major Abdominal Surgery: A Randomized Controlled Trial. Ann Surg 2016; 264(1): 41-6.

36. Unger EF, Thompson AM, Blank MJ, Temple R. Erythropoiesis-stimulating agents--time for a reevaluation. N Engl J Med 2010; 362(3): 189-92.

37. Tankiewicz-Kwedlo A, Hermanowicz J, Surazynski A, et al. Erythropoietin accelerates tumor growth through increase of erythropoietin receptor (EpoR) as well as by the stimulation of angiogenesis in DLD-1 and Ht-29 xenografts. Mol Cell Biochem 2016; 421(1-2): 1-18.

38. Ferreira V, Agnihotram RV, Bergdahl A, van Rooijen SJ, Awasthi R, Carli F, Scheede-Bergdahl C. Maximizing patient adherence to prehabilitation: what do the patients say? Support Care Cancer 2018; 26(8): 2717-23.

39. Looijaard SMLM, Slee-Valentijn MS, Otten RHJ, Maier AB. Physical and Nutritional Prehabilitation in Older Patients With Colorectal Carcinoma: A Systematic Review. J Geriatr Phys Ther 2018; 41(4): 236-44.

40. Strous MTA, Janssen-Heijnen MLG, Vogelaar FJ. Impact of therapeutic delay in colorectal cancer on overall survival and cancer recurrence - is there a safe timeframe for prehabilitation? Eur J Surg Oncol 2019; 45(12): 2295-301.

41. Charlson M, Szatrowski TP, Peterson J, Gold J. Validation of a combined comorbidity index. J Clin Epidemiol 1994; 47(11): 1245-51.

42. Slankamenac K, Graf R, Barkun J, Puhan MA, Clavien PA. The comprehensive complication index: a novel continuous scale to measure surgical morbidity. Ann Surg 2013; 258(1): 1-7. 


\section{Appendix}

\section{Appendix 7.I}

\section{Methods}

\section{Intervention}

Each 60-minute training session consisted of moderate-to-high intensity interval training on a cycle ergometer (TechnoGym, Bike Med, Gambettola, Italy) to improve aerobic fitness (40 minutes), and resistance training to improve peripheral muscle strength (20 minutes). CPET results were used to personalize training. The interval training component included a 7-minute warm-up at 50\% of the work rate achieved at the VAT at the baseline CPET, 30 minutes of interval training, and a 3-minute cool-down at 50\% of the work rate achieved at the VAT. Interval training consisted of six 5-minute intervals in which moderate-to-highintensity exercise at $120 \%$ of the work rate achieved at the VAT was alternated with active recovery at $50 \%$ of the work rate achieved at the VAT. In the first week, exercise lasted 120 seconds and recovery lasted 180 seconds, which progressed to 140 and 160 seconds, respectively, in the second week, and 160 and 140 seconds in the third week. The duration of exercise was increased according to the participant's perceived exertion to maximize the training effect. Participants were asked to maintain a pedaling frequency throughout the interval training sessions of 60 to 80 revolutions/min.

Peripheral resistance training of the large muscle groups of the lower and upper extremities consisted of squat exercises (primary muscle group, quadriceps femoris; secondary muscle groups, gluteal muscles, hamstring muscles, and gastrocnemius muscle), and exercises with a pulley to train pulling (primary muscle groups, latissimus dorsi muscle and rhomboid muscles; secondary muscle groups, biceps brachii muscle, rotator cuff, and trapezius muscle), pushing (primary muscle group, pectoral muscles; secondary muscle groups, shoulder muscles, triceps brachii muscle), and lifting (primary muscle groups, trunk muscles and shoulder muscles; secondary muscle groups, quadriceps femoris, gluteal muscles, and trapezius muscle). Participants performed three sets of eight repetitions for each muscle group, with rest intervals of 60 to 90 seconds, at a personalized intensity of $70 \%, 76 \%$, and $82 \%$ of their baseline one-repetition maximum in the first, second, and third week, respectively. At baseline, each patient's one-repetition maximum was estimated from the patient's 10-repetition maximum using the Oddvar Holten diagram. ${ }^{1}$

Additionally, patients performed unsupervised exercises at home at a moderate exercise intensity (e.g., walking, cycling, sit-to-stand exercises, or stair climbing) twice a week for at least 30 minutes. This was monitored by means of a patient diary checked by the community physical therapist. 


\section{Reference appendix 7.I}

1. Hertling D, Kessler RM. Management of common musculoskeletal disorders: physical therapy principles and methods. Philadelphia: Lippincott Williams \& Wilkins, 2006. 


\section{Appendix 7.2}

\section{Methods}

\section{Outcomes}

During the CPET, absolute values at peak exercise were calculated as the average value over the last 30 seconds prior to test termination. Peak heart rate was defined as the highest heart rate achieved during the CPET. A CPET was considered to be at or near the maximal level when at least one of the following criteria was met: a heart rate at peak exercise of $>95 \%$ of predicted (predicted peak heart rate [beats $/ \mathrm{min}$ ] $=208-0.7 \times$ age [years]) and/ or a respiratory exchange ratio at peak exercise of $>1.10$. Aerobic fitness was assessed by determining the $\mathrm{VO}_{2}$ at the VAT and at peak exercise $\left(\mathrm{VO}_{2 \text { peak }}\right)$. The VAT was detected by using the V-slope method ${ }^{1}$ and verified with the ventilatory equivalents method. ${ }^{2}$

\section{References appendix $\mathbf{7 . 2}$}

1. Beaver WL, Wasserman K, Whipp BJ. A new method for detecting anaerobic threshold by gas exchange. J Appl Physiol (1985) 1986; 60(6): 2020-7.

2. American Thoracic Society, American College of Chest Physicians. ATS/ACCP Statement on cardiopulmonary exercise testing. Am J Respir Crit Care Med 2003; 167(2): 211-77. 


\section{Appendix 7.3}

\section{Methods}

\section{Sample size calculation}

Our interim analysis showed a significantly lower incidence of complications ( $33 \%$ versus $73 \% ; p=0.0096)$ in patients in the prehabilitation group $(n=21)$ than in patients in the usual care group ( $n=22$ ). Although the significance ( $p$-value) of this difference was not $<0.0054$, the difference was obvious and clinically very relevant. Moreover, patient inclusion was challenging. It took over three years to include half of the patients (43 patients) needed for the interim analysis. Therefore, we deliberated with the medical ethics committee about the repercussions of our interim analysis. The medical ethics committee proposed a new sample size calculation. Therefore, we recalculated our sample size with the hypothesis of a complication rate of $33 \%$ in the prehabilitation group and a complication rate of $73 \%$ in the usual care group, and now needed 27 patients in each group to detect statistically significant differences between groups ( $\alpha$ of 0.0492 , $ß$ of $80 \%$, taking a $10 \%$ dropout rate into account). If the interim analysis would have shown no or minimal difference $(<10 \%$ on overall complications), the study would have stopped due to futility. 



\section{Discussion}

\section{Preface}

Colorectal cancer is the third most common cancer worldwide, with a total estimated caseload of 1.8 million per year. ${ }^{1}$ In the Netherlands, the incidence of colorectal cancer was 14,000 till almost 16,000 per year in $2014-2018 .^{2}$ In 2020, the incidence was 12,000 , which is lower than the previous years due to the national colorectal screening program and the covid-19 pandemic. ${ }^{3}$ Surgical resection of the tumor remains the cornerstone of curative treatment. ${ }^{4}$ Despite substantial improvements in perioperative outcomes in the last decades, such as the introduction of minimal invasive surgical techniques within enhanced recovery after surgery (ERAS) programs, the national mandatory quality registry for colorectal cancer (Dutch ColoRectal Audit, DCRA), the national colorectal screening program, and concentration of care, the overall complication rate after colorectal surgery remains significant with percentages up to $30 \% .^{4-6}$ What is of special interest here is that patients with a complicated postoperative course tend to be less physically fit preoperatively. ${ }^{7,8}$ Moreover, less physically fit patients are known to be more vulnerable for all sorts of complications and their consequences, amongst others due to a reduced ability to meet the increased oxygen demand during and after surgery. ${ }^{9}$ Therefore, preoperative risk assessment remains of high importance to timely identify patients with an increased risk of postoperative complications. By doing so, modifiable risk factors can be detected in time and intervened upon as soon as possible, as it is known that perioperative outcome can be improved by optimization of these modifiable risk factors. Therefore, the aim of this thesis was to find tools to screen, assess, and diagnose highrisk patients with colorectal cancer throughout the preoperative period (with the focus on physical fitness in the preoperative phase) and to investigate if postoperative outcomes of these patients can be improved with a targeted community-based and physical exercise training focused prehabilitation program. In this chapter, the main results of this thesis will be aggregated, interpreted, and discussed ('what's new') in the context of the existing clinical practice and literature ('what's known'). At last, recommended steps to take in the (near) future ('what's next') will be discussed.

\section{What's known?}

Diagnosis, hospitalization, and colorectal resection of a tumor can have a considerable impact on a patient and also on his or her close relatives and (in)formal support system. Approximately one-third of patients who undergo colorectal resection experience diverse postoperative complications. ${ }^{5,6}$ Complications and their consequences can delay recovery, prolong hospitalization, cause unplanned hospital readmission and chronic illness, and severely impair short- and long-term physical functioning and health-related quality of life. ${ }^{10}$ Reducing complications (tertiary prevention) would considerably improve benefits of diagnosis, hospitalization, and surgery, and at the same time reduce costs. After colorectal 
cancer surgery in the Netherlands, minor and severe complications were respectively associated with $26 \%(\sim+€ 2,400)$ and 196\% ( +€18,000) higher costs (primary admission and after discharge up to 90 days) as compared to patients without complications. ${ }^{5}$ In recent years, preoperative risk screening and assessment has gained more and more interest to identify patients at high and low risk of postoperative complications. Known risk factors that could assist in identifying patients with a potential risk for an impaired postoperative course, which are already regularly used as case-mix correction factors in the DCRA, are sex, body mass index (BMI), age, Charlson comorbidity score, American Society of Anesthesiologists (ASA) classification, preoperative tumor complications (e.g. fistula, obstruction, perforation, anaemia), urgency of the resection, tumor location, extensive resection due to locally advanced tumors or metastases, pathologic tumor ( $\mathrm{pT}$ ) stage, and pathologic metastasis (pM) stage. ${ }^{11}$ However, a patient's body composition and preoperative aerobic fitness might be additional or even more powerful preoperative risk assessment tools to diagnose and thereupon identify high-risk patients and to predict and, even more importantly, adjust their postoperative outcome after major abdominal surgery.

The computed tomography (CT) scan, which is part of usual care in the diagnostic workup of colorectal cancer patients, can be used to assess specific derivate indicators of a patient's body composition (i.e., the relative proportions of subcutaneous adipose tissue, visceral adipose tissue, skeletal muscle quantity and quality). Heymsfield and colleagues were, between 1979 and 1981, to our knowledge the first to report the use of the CT scan to measure skeletal muscle mass, visceral organ volumes, and visceral adipose tissue. ${ }^{12}$ In the recent years, body composition has extensively been studied as a predictor for outcomes following oncological treatment. ${ }^{13}$ As an indicator of body composition, sarcopenia (i.e. the involuntary, age-dependent, depletion of skeletal muscle mass and reduction of skeletal muscle function) ${ }^{14}$ was studied massively and was found to be associated with more infectious complications, ${ }^{15}$ higher mortality, ${ }^{16}$ and decreased survival ${ }^{17}$ after colorectal cancer surgery. Furthermore, a lower skeletal muscle radiation attenuation was found to be a significant predictor of surgical complications after colectomy for colon cancer, ${ }^{18}$ and was an independent prognostic determinant of survival of patients with cancer. ${ }^{19}$ Moreover, sarcopenic obesity, in which (severe) obesity and low muscle mass occur simultaneously, ${ }^{13}$ was associated with severe postoperative complications after open colon cancer resection, ${ }^{20}$ and was found to be an independent predictor of survival of patients with respiratory or gastrointestinal tract tumors. ${ }^{13}$

In the literature another powerful preoperative risk assessment tool that has consistently been found to be able to independently predict postoperative outcome of patients undergoing intra-abdominal surgery, is preoperative aerobic fitness. ${ }^{7}$ Patients with a low preoperative aerobic fitness (oxygen uptake $\left(\mathrm{VO}_{2}\right)$ at the ventilatory anaerobic threshold 
(VAT) $<11 \mathrm{~mL} / \mathrm{kg} / \mathrm{min}$, or a $\mathrm{VO}_{2}$ at peak exercise $\left.\left(\mathrm{VO}_{2 \text { peak }}\right)<18 \mathrm{~mL} / \mathrm{kg} / \mathrm{min}\right)$ as assessed by cardiopulmonary exercise testing (CPET)) were found to be particularly at increased risk of an impaired postoperative outcome. ${ }^{7,8}$

Hospitalization and surgery cause changes in hormonal, hematological, metabolic, and immunological metabolism. The magnitude of this perioperative metabolic activation depends on the magnitude of the precipitation of diagnosis, hospitalization, and specifically of the impact of the surgical procedure. Additionally, it also depends on the patient's ability to adapt to these stressors in order to biologically cope with this complex of allostatic stressors via homeostatic and allostatic counteractions. For instance, the complex of metabolic stress responses are necessary to successfully overcome a diagnosis, hospitalization, and particularly surgical trauma. ${ }^{9}$ When high-risk patients are diagnosed and modifiable risk factors are determined, targeted preventive strategies could be considered and motivated and, upon shared decision with the patient, initiated to improve his/her capacity to physically - and probably also mentally and socially - cope with the consequences of diagnosis, hospitalization, and surgery, with the ultimate goal to improve their postoperative outcome. These high-risk patients might benefit from preoperative physical exercise training, a paramount part of prehabilitation.

To date, exercise prehabilitation programs have been shown to improve physical fitness before hospitalization and surgery. However, evidence that this reduces postoperative complications still seems inconclusive..$^{10,21-24}$ When focused on adequate diagnosis and selection of high-risk surgical patients, the systematic review of Thomas and colleagues ${ }^{21}$ found that prehabilitation of patients before hospitalization and surgery for major intraabdominal cancer improved postoperative outcomes.

In the seventies, eighties, and nineties of the previous century, prehabilitation gained interest, especially in patients undergoing cardiac surgery. Studies showed that preoperative respiratory physical therapy (inspiratory muscle training) may have advantages over postoperative care in patients undergoing cardiac surgery, especially in reducing (some) postoperative pulmonary complications..$^{25-28}$ Until 2006 however, most studies performed were often of low methodological quality and low therapeutic quality (i.e. inadequate rationale, inadequate patient selection [inclusion of relatively healthy patients rather than high-risk patients], inadequate content and context). ${ }^{29-32}$ Probably the first adequately powered trial focusing on high-risk coronary artery bypass graft (CABG) patients demonstrated the potential of a preoperative inspiratory muscle training program, lowering the postoperative pulmonary complications with almost $60 \% .{ }^{29} \mathrm{In}$ the recent years, there is an almost exponentially increasing interest in the scientific research of prehabilitation programs to optimize patient- and treatment-related outcomes after major surgery (Figure 1). It was learned that preoperative interventions should particularly 
be tailored to high-risk patients. ${ }^{21,33,34}$ Moreover, to maximize participation rate, adherence (both known to be challenging), and effectiveness in high-risk patients, a prehabilitation program must ideally be performed within the patient's own living situation and probably supported by relatives and/or (in)formal caregivers. ${ }^{35}$ For the right clinical validity of physical exercise training interventions, also in the context of prehabilitation, Hoogeboom et al. ${ }^{31,32}$ developed a rating scale, the CONTENT-scale (and, more recently, the i-CONTENTscale) that is nowadays more and more used to develop a remedy with more adequate potential in advance.

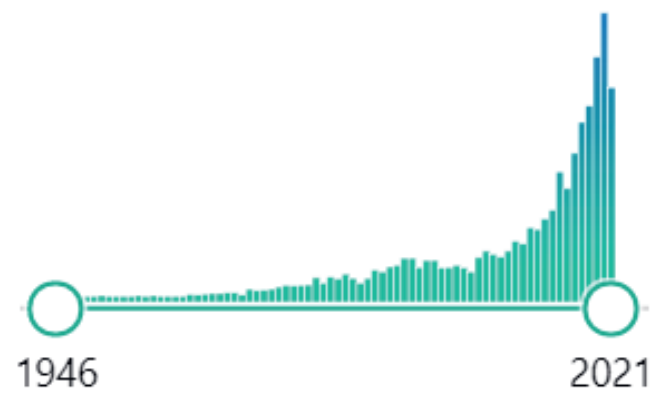

Figure 1 Exponentially grow in results on PubMed keyword 'Prehabilitation' per year.

\section{What's new?}

In this thesis, we first tried to find and validate tools to select high-risk patients with colorectal cancer at the earliest point in time of the preoperative period (Chapter 2-5). To gain more insight in the 'traditional' factors that are associated with postoperative complications and pathologic complete response ( $\mathrm{PCR}$ ) in patients with locally advanced rectal cancer treated with neoadjuvant chemoradiation followed by surgery, we retrospectively aimed to identify and describe these prognostic factors (Chapter 2). This, in order to help patients, their relatives, and the clinicians with determining prognosis and, thereupon, eventually select the proper treatment choices via shared decisions on interventions like prehabilitation. We found that age, intra- or postoperative blood transfusions, and pathologic tumor (ypT) stage predicted postoperative complications and/or postoperative grade 3-5 complications. No independent factors predicting pCR were found on the base of this approach.

In Chapter 3, the associations between body composition as assessed by using the CT scan, possibly a more powerful preoperative risk assessment tool, and postoperative outcome after treatment of locally advanced rectal cancer were evaluated. We found that radiation attenuation was associated with overall and grade 3-5 postoperative morbidity. Moreover, sarcopenic obesity was associated with overall complications. This study underscored the importance of assessing body composition in the context of the diagnosis of patients with 
high risk of worse outcome after surgery. These patients might benefit from preoperative optimization (prophylactic prehabilitation) to optimize perioperative health status and to consequently possibly prevent a dismal course. Since taking part in a CT scan is a usual ingredient of care in the work-up of all patients with rectal cancer, the advantage of using body composition as a risk stratification tool is that no additional test is needed when considering body composition with regard to the preoperative screening, assessment, and interpretation of risks.

As described before, aerobic (cardiorespiratory) fitness, most commonly indicated by $\mathrm{VO}_{2 \text { peak }}$ and/or $\mathrm{VO}_{2}$ at the VAT on the CPET, is a widely recognized important measure in the evaluation of the risk of perioperative complications in major abdominal surgery. The measurement of $\mathrm{VO}_{2 \text { eak }}$, however, can be strongly influenced by the patient's motivation to deliver a maximal effort, and might be invalid in patients whose performance may be limited because of pain or fatigue rather than cardiorespiratory deficiency. Besides $\mathrm{VO}_{2 \text { peak}}$, also the $\mathrm{VO}_{2}$ at the VAT cannot always be determined, and its values has been reported to depend on the choice of ergometer, exercise protocol, and method of detection. ${ }^{36-39}$ Additionally, only focusing on physiological parameters at specific time points leads to the loss of a significant amount of data generated during a CPET that might better reflect the continuous, dynamic cardiopulmonary responses throughout the test. Due to these clinical limitations when determining aerobic fitness using $\mathrm{VO}_{2 \text { peak }}$ and/or $\mathrm{VO}_{2}$ at the VAT, alternative objective (sub)maximal variables of aerobic fitness (e.g., the oxygen uptake efficiency slope, OUES) might be useful for preoperative risk assessment. However, the validity of the OUES as a patient's effort-independent alternative measure of preoperative aerobic fitness, and its ability to predict postoperative outcomes, were still unknown. Therefore, we aimed to investigate the validity of the OUES as an effort-independent indicator of aerobic fitness of elderly patients scheduled for colorectal surgery, by correlating it with $\mathrm{VO}_{2 \text { peak }}$ and the $\mathrm{VO}_{2}$ at the VAT and assessing its stability throughout the last part of the CPET (Chapter 4). The OUES was calculated up to different exercise intensities, using $100 \%, 90 \%$, and $80 \%$ of the exercise data. We showed that the OUES provides an objective and valid measure of aerobic fitness of these patients, as indicated by the high correlation coefficients with $\mathrm{VO}_{2 \text { peak }}\left(0.816\right.$ to 0.825 , all $p<0.001$ ) and the $\mathrm{VO}_{2}$ at the VAT $(0.793$ to 0.805 , all $p<0.001)$. Moreover, its ability to predict cut-off points for $\mathrm{VO}_{2 \text { peak }}$ and $\mathrm{VO}_{2}$ at the VAT might help to diagnose and discriminate (respectively in the context of the clinic and that of research) patients at higher risk of postoperative morbidity.

Since the CPET is relatively expensive, time-consuming, and requires well-trained personnel for an adequate interpretation of its results, we were also interested in whether body composition parameters on CT scan could help to diagnose and - in the context of research - select patients with a potentially low preoperative aerobic fitness. By doing so, we might be able to appropriately refer the right patients for performing 
a CPET. Therefore, in Chapter 5, we evaluated the association between preoperative body composition and aerobic fitness of patients scheduled for colorectal surgery. We found that body composition variables of the included 78 patients were moderately associated with preoperative CPET-derived aerobic fitness. A higher ASA score and a higher visceral adipose tissue mass were associated with an increased risk of a VAT $\leq 11.1$ $\mathrm{mL} / \mathrm{kg} / \mathrm{min}$ as a cut-off to classify patients as having an increased risk for postoperative morbidity, based on the study of West and colleagues. ${ }^{8}$ We concluded that the routinely performed abdominal CT scan cannot replace the CPET for preoperative diagnosis and/ or risk assessment; however, it may contribute to the diagnosis, and (pre)selection of less physically fit patients and thereupon shared decisions for taking part in prehabilitation interventions.

Next, we were interested whether these high-risk, less physically fit patients would benefit from taking part in preoperative exercise training as a part of prehabilitation in order to reduce the risks of developing postoperative complications. Therefore, we performed an adequately powered randomized controlled trial to evaluate the effects of a three-week prehabilitation program on 30-day postoperative complications with patients with a VAT $<11 \mathrm{~mL} / \mathrm{kg} / \mathrm{min}$ planned for elective colorectal resection for colorectal cancer or dysplasia (Chapter 7). In Chapter 6 the study protocol was extensively described, providing those who are interested or involved in prehabilitation an early-stage insight in the study procedures at the beforehand. In Chapter 7, by executing this protocol we showed that communitybased exercise prehabilitation reduced the risk of postoperative complications (with a RR of 0.59 ) of high-risk patients scheduled for elective colon resection for (pre)malignancy. That is: within a relatively short period of three weeks, prehabilitation carried out by these patients under supervision of a well-trained physical therapist improved aerobic fitness by on average approximately $10 \%$ and the patients in the prehabilitation group demonstrated a more than $40 \%$ absolute reduction of the incidence of postoperative complications when compared to the complication rate of the usual care group. We also showed by post hoc analysis that patients, situated in both the prehabilitation as well as the usual care group, with a lower preoperative hemoglobin level were at a greater risk of postoperative complications. Therefore, we suggested that, in the future, preoperative hemoglobin level should also be taken into consideration when diagnosing the risk-profile of patients, and thereupon optimization of preoperative hemoglobin level should in our opinion definitely be considered. In this study, we experienced that patient inclusion in the trial was extremely challenging as 57 patients out of 132 eligible patients were included over a period of 4.5 years. In our study, we experienced a range of reasons why patients were either unable or declined to participate, e.g., no inclination to participate, inability to perform cycling exercises, considered self to be appropriately physically fit already, or the desire for surgery as soon as possible. We have learned from these reasons during the trial and from the in recent years vastly growing amount of prehabilitation experiences 
and literature. Therefore, we concluded that in order to maximize participation rate and adherence, a preoperative exercise program might best be integrated in the perioperative trajectory and performed in the patient's pre-existent living context, thereby (partly) supervised by a competent community physical therapist and involving the patient's informal support system. ${ }^{21,33}$ Moreover, as a side-effect of executing this study, hospital logistics were optimized (e.g., a shorter period between time of diagnosis and first visit at the outpatient surgical clinic) and a more optimal collaboration between all disciplines was realized. We consider these side effects as essential for the proper inclusion of patients in trials as these, and probably also in the real life daily clinical context of prehabilitation. We concluded that from the moment these study results were obvious, prehabilitation should preferably be implemented and thereby considered as an instantaneous part of usual care with high-risk patients scheduled for elective colon surgery. Probably the same considerations should account for those patients that are diagnosed with rectal cancer and preparing for surgery, despite the small number of rectal cancer patients $(n=3)$ included here.

The findings of this thesis can be used to adequately diagnose and advise patients at high risk about their regretfully more substantial chances of an impaired postoperative course and the options to optimize their modifiable risk factors, and to consequently improve their chances lowering their risks of adverse postoperative outcomes with help of a personalized exercise prehabilitation program that is heavily based on evidence such as presented here in combination with good clinical practice.

\section{What's next?}

Now that we have learned more about body composition (derived from CT scans) and aerobic fitness (measured with CPET) as possible risk assessment tools to diagnose, advise and, if appropriate, select high-risk patients with colorectal cancer throughout the preoperative period and the positive effects of prehabilitation in reducing the risk of postoperative complications with these high-risk patients, there are a few next steps to take. A next step is to implement risk assessment with body composition analyzed at the third lumbar vertebra (L3) level of the CT scan and use it as a case-mix correction factor in the DCRA to make a more accurate comparison of outcome among various hospitals. Moreover, prehabilitation programs should be further improved, and prehabilitation as part of the new/innovative standard care should be implemented and obsolete ingredients (e.g., as still frequently by formal and informal caregivers advocated passive coping styles as (bed) rest) should be de-implemented. Another step is to focus on the medium- and long-term effects of prehabilitation and performing a benefit-cost-effectiveness analysis in combination with a profit and loss analysis of prehabilitation for colorectal surgery. Future research should also address the prognostic value of the OUES for different postoperative outcomes in major abdominal surgery. 
In our research, the prehabilitation program was a unimodal program (physical exercise training). In future research, multimodal programs that consider for example physical exercise training, nutritional support, psychological support, smoking and alcohol cessation interventions, optimization of preoperative hemoglobin level, and the interaction between these components should be considered, since these programs might be more or even most effective. ${ }^{9,21}$ Currently, definitive clinical evidence of multimodal prehabilitation is very limited, ${ }^{21,40}$ but at the beforehand its potential seems logical on the base of the ample room for further improvement shown in this thesis. We infer that to further improve the (effects of the) prehabilitation program, prehabilitation should be personalized, (partly) supervised, home- or community-based, and progression should be objectively monitored and training intensity should be adjusted accordingly. Also, in future research, attempts should be made to ensure that all eligible high-risk patients are willing and able to participate in prehabilitation programs, when and where necessary supported by their relatives and caregivers, to create a proactive culture throughout the continuum of care at home as well as in the care setting to keep all patients as active as possible, physically, mentally, and socially. ${ }^{41}$

Not only the effect of prehabilitation on the incidence of postoperative complications should be evaluated, but also the impact of a postoperative complication in individual patients. Patients with a higher physical fitness level might of course still experience postoperative complications, but the impact of a postoperative complication might be mitigated compared to patients with a lower level of physical fitness. ${ }^{9,21,29,42}$ Patients with a higher physical fitness level in that line of reasoning may biologically cope better with the stressors of the perioperative process and of a complication. Outcome measures should be developed in which not just the complication as such is scored, but all the more the complication and its impact is estimated, for example by combining a complication score for each individual patient with its attributed impact on the - extra - use of resources, length of hospital stay, time to recovery of physical functioning, and time to return to normal activities and participation.

Community-based perioperative care networks and in-hospital prehabilitation departments should be established, in which trained and competent healthcare professionals, along with the patient and the patient's informal support system, aim to perform a multidisciplinary preoperative risk assessment and to assist the patient to get fit for surgery. The ultimate goal will be to achieve the quadruple aim ${ }^{43}$ : improving the patient performance outcomes and their perceptions and experiences about the care provided (including quality and satisfaction), improving the health of a patient and or populations of patients, reducing the per capita cost of health care, and improving the perceptions of the - contributions of healthcare professionals in these processes. 
Based on the findings of this thesis, we would suggest that prehabilitation should be stepwise implemented, monitored, and evaluated in usual care in adequately diagnosed high-risk patients that decide to participate in prehabilitation prior to elective colorectal surgery.

\section{Final conclusions}

The studies described in this thesis highlight the role of body composition as a tool to preoperatively diagnose and/or select patients scheduled for colorectal surgery who are at risk for postoperative complications. Moreover, body composition was found to be moderately associated with aerobic fitness of these patients. We also found that the OUES might be an objective and valid CPET measure of aerobic fitness in elderly patients scheduled for major colorectal surgery, which might - besides the $\mathrm{VO}_{2}$ at the VAT - also help to discriminate patients at higher risk of postoperative morbidity. Finally, we showed that a three-week community-based exercise prehabilitation program substantially reduced the risk of postoperative complications in high-risk patients scheduled for elective colon resection for (pre)malignancy. Future research in the field of prehabilitation should focus on developing and implementing tailored (multimodal) prehabilitation programs for the right patients, in the right context, and using the right outcome measures. 


\section{References}

1. Bray F, Ferlay J, Soerjomataram I, Siegel RL, Torre LA, Jemal A. Global cancer statistics 2018: GLOBOCAN estimates of incidence and mortality worldwide for 36 cancers in 185 countries. CA Cancer J Clin 2018; 68(6): 394-424.

2. Integraal Kankercentrum Nederland (IKNL). Nederlandse Kanker Registratie cijfers. Available at: https://www.iknl.nl/nkr-cijfers. Last accessed May 4, 2021.

3. Integraal Kankercentrum Nederland (IKNL). Nederlands Kanker Registratie cijfers. Cijfers darmkanker 2020; Available at: https://iknl.nl/nieuws/2021/cijfers-darmkanker-2020. Last accessed May 4, 2021.

4. Dutch institute for clinical auditing (DICA) leiden. DICA jaarrapportage 2018: Dutch ColoRectal Audit (DCRA). Available at: https://dica.nl/jaarrapportage-2018/dcra. Last accessed April 20, 2020.

5. Govaert JA, Fiocco M, van Dijk WA, et al. Costs of complications after colorectal cancer surgery in the Netherlands: Building the business case for hospitals. Eur J Surg Oncol 2015; 41(8): 1059-67.

6. Tevis SE, Kennedy GD. Postoperative Complications: Looking Forward to a Safer Future. Clin Colon Rectal Surg 2016; 29(3): 246-52.

7. Moran J, Wilson F, Guinan E, McCormick P, Hussey J, Moriarty J. Role of cardiopulmonary exercise testing as a risk-assessment method in patients undergoing intra-abdominal surgery: a systematic review. Br J Anaesth 2016; 116(2): 177-91.

8. West MA, Asher R, Browning M, et al. Validation of preoperative cardiopulmonary exercise testing-derived variables to predict in-hospital morbidity after major colorectal surgery. $\mathrm{Br} J$ Surg 2016; 103(6): 744-52.

9. Bongers $\mathrm{BC}$, Dejong $\mathrm{CHC}$, den Dulk M. Enhanced recovery after surgery programmes in older patients undergoing hepatopancreatobiliary surgery: what benefits might prehabilitation have? Eur J Surg Oncol 2020; https://doi.org/10.1016/j.ejso.2020.03.211.

10. Moran J, Guinan E, McCormick P, et al. The ability of prehabilitation to influence postoperative outcome after intra-abdominal operation: A systematic review and meta-analysis. Surgery 2016; 160(5): 1189-201.

11. Dutch institute for clinical auditing (DICA) leiden. Factsheet indicatoren Dutch ColoRectal Audit (DCRA) 2019. Available at: https://dica.nl/dcra/documenten. Last accessed June 8, 2020.

12. Heymsfield SB, Wang Z, Baumgartner RN, Ross R. Human body composition: advances in models and methods. Annu Rev Nutr 1997; 17: 527-58.

13. Prado CM, Lieffers JR, McCargar LJ, Reiman T, Sawyer MB, Martin L, Baracos VE. Prevalence and clinical implications of sarcopenic obesity in patients with solid tumours of the respiratory and gastrointestinal tracts: a population-based study. Lancet Oncol 2008; 9(7): 629-35.

14. Rolland Y, Abellan van Kan G, Gillette-Guyonnet S, Vellas B. Cachexia versus sarcopenia. Curr Opin Clin Nutr Metab Care 2011; 14(1): 15-21. 
15. Lieffers JR, Bathe OF, Fassbender K, Winget M, Baracos VE. Sarcopenia is associated with postoperative infection and delayed recovery from colorectal cancer resection surgery. $\mathrm{Br} J$ Cancer 2012; 107(6): 931-6.

16. Reisinger KW, van Vugt JL, Tegels JJ, et al. Functional compromise reflected by sarcopenia, frailty, and nutritional depletion predicts adverse postoperative outcome after colorectal cancer surgery. Ann Surg 2015; 261(2): 345-52.

17. Miyamoto Y, Baba Y, Sakamoto Y, et al. Sarcopenia is a Negative Prognostic Factor After Curative Resection of Colorectal Cancer. Ann Surg Oncol 2015; 22(8): 2663-8.

18. Sabel MS, Terjimanian M, Conlon AS, et al. Analytic morphometric assessment of patients undergoing colectomy for colon cancer. J Surg Oncol 2013; 108(3): 169-75.

19. Martin L, Birdsell L, Macdonald N, et al. Cancer cachexia in the age of obesity: skeletal muscle depletion is a powerful prognostic factor, independent of body mass index. J Clin Oncol 2013; 31(12): 1539-47.

20. Boer BC, de Graaff F, Brusse-Keizer M, Bouman DE, Slump CH, Slee-Valentijn M, Klaase JM. Skeletal muscle mass and quality as risk factors for postoperative outcome after open colon resection for cancer. Int J Colorectal Dis 2016; Jun;31(6): 1117-24.

21. Thomas G, Tahir MR, Bongers BC, Kallen VL, Slooter GD, van Meeteren NL. Prehabilitation before major intra-abdominal cancer surgery: A systematic review of randomised controlled trials. Eur J Anaesthesiol 2019; 36(12): 933-45.

22. Bruns ER, van den Heuvel $B$, Buskens $C J$, et al. The effects of physical prehabilitation in elderly patients undergoing colorectal surgery: a systematic review. Colorectal Dis 2016; 18(8): O26777.

23. Hijazi Y, Gondal U, Aziz O. A systematic review of prehabilitation programs in abdominal cancer surgery. Int J Surg 2017; 39: 156-62.

24. Waterland JL, McCourt O, Edbrooke L, Granger CL, Ismail H, Riedel B, Denehy L. Efficacy of Prehabilitation Including Exercise on Postoperative Outcomes Following Abdominal Cancer Surgery: A Systematic Review and Meta-Analysis. Front Surg 2021; 8: 628848.

25. Vraciu JK, Vraciu RA. Effectiveness of breathing exercises in preventing pulmonary complications following open heart surgery. Phys Ther 1977; 57(12): 1367-71.

26. Castillo R, Haas A. Chest physical therapy: comparative efficacy of preoperative and postoperative in the elderly. Arch Phys Med Rehabil 1985; 66(6): 376-9.

27. Rajendran AJ, Pandurangi UM, Murali R, Gomathi S, Vijayan VK, Cherian KM. Preoperative short-term pulmonary rehabilitation for patients of chronic obstructive pulmonary disease undergoing coronary artery bypass graft surgery. Indian Heart J 1998; 50(5): 531-4.

28. Weiner P, Zeidan F, Zamir D, Pelled B, Waizman J, Beckerman M, Weiner M. Prophylactic inspiratory muscle training in patients undergoing coronary artery bypass graft. World J Surg 1998; 22(5): 427-31.

29. Hulzebos EH, Helders PJ, Favie NJ, De Bie RA, Brutel de la Riviere A, Van Meeteren NL. Preoperative intensive inspiratory muscle training to prevent postoperative pulmonary 
complications in high-risk patients undergoing CABG surgery: a randomized clinical trial. JAMA 2006; 296(15): 1851-7.

30. Pasquina $P$, Tramèr $M R$, Walder $B$. Prophylactic respiratory physiotherapy after cardiac surgery: systematic review. BMJ 2003; 327(7428): 1379.

31. Hoogeboom TJ, Oosting E, Vriezekolk JE, et al. Therapeutic validity and effectiveness of preoperative exercise on functional recovery after joint replacement: a systematic review and meta-analysis. PLoS One 2012; 7(5): e38031.

32. Hoogeboom TJ, Kousemaker MC, van Meeteren NL, et al. i-CONTENT tool for assessing therapeutic quality of exercise programs employed in randomised clinical trials. Br J Sports Med 2020; doi: 10.1136/bjsports-2019-101630.

33. Bongers BC, Punt IM, van Meeteren NL. On "Prehabilitation: The emperor's new clothes or a new arena for physical therapists?" Lundberg M, Archer KR, Larsson C, Rydwik E. Phys Ther. 2018;12:127-130. Phys Ther 2019; 99(7): 953-4.

34. Tew GA, Ayyash R, Durrand J, Danjoux GR. Clinical guideline and recommendations on preoperative exercise training in patients awaiting major non-cardiac surgery. Anaesthesia 2018; 73(6): 750-68.

35. Bongers BC, Dronkers JJ, Hulzebos HJ, Hoogeboom TJ, Buhre WF, van Meeteren NL. Optimizing perioperative physical therapy care in major elective surgery to improve surgical outcome in high-risk patients: the Better in, Better out ${ }^{\mathrm{TM}}$ concept. Ned Tijdschr Anesthesiol 2016;29:134-9.

36. Meyer K, Hajric R, Westbrook S, et al. Ventilatory and lactate threshold determinations in healthy normals and cardiac patients: methodological problems. Eur J Appl Physiol Occup Physiol 1996; 72(5-6): 387-93.

37. Vanhees L, Lefevre J, Philippaerts R, Martens M, Huygens W, Troosters T, Beunen G. How to assess physical activity? How to assess physical fitness? Eur J Cardiovasc Prev Rehabil 2005; 12(2): 102-14.

38. Akkerman M, van Brussel M, Hulzebos E, Vanhees L, Helders PJ, Takken T. The oxygen uptake efficiency slope: what do we know? J Cardiopulm Rehabil Prev 2010; 30(6): 357-73.

39. Shimizu M, Myers J, Buchanan N, Walsh D, Kraemer M, McAuley P, Froelicher VF. The ventilatory threshold: method, protocol, and evaluator agreement. Am Heart J 1991; 122(2): 509-16.

40. Looijaard SMLM, Slee-Valentijn MS, Otten RHJ, Maier AB. Physical and Nutritional Prehabilitation in Older Patients With Colorectal Carcinoma: A Systematic Review. J Geriatr Phys Ther 2018; 41(4): 236-44.

41. Hulzebos EH, van Meeteren NL. Making the elderly fit for surgery. Br J Surg 2016; 103(4): 463.

42. Van Beijsterveld CAFM, Bongers BC, Den Dulk M, Van Kuijk SMJ, Dejong CHC, Van Meeteren NLU. Exploring the relation between preoperative physical functioning and the impact of major complications in patients following pancreatic resection. HPB (Oxford) 2020; 22(5): 71627.

43. Bodenheimer T, Sinsky C. From triple to quadruple aim: care of the patient requires care of the provider. Ann Fam Med 2014; 12(6): 573-6. 



\section{Summary}

The number of the elderly in the population is rapidly growing, as overall life expectancy continues to increase. With the rise of the aging population, the incidence of cancer will also increase. In colorectal cancer, surgical resection of the tumor, with or without (neo)adjuvant treatment, remains the foundation of curative treatment. In perioperative care, there is an increased focus on prevention, for example on reducing (the impact of) postoperative complications and on preventing a delayed or even permanent loss of (physical) functioning. Despite substantial improvements in perioperative outcomes in the last decades, the overall complication rate after colorectal surgery remains significant with percentages up to $30 \%$. A complicated postoperative course may delay or even hamper recovery, prolongs hospitalization, causes unplanned hospital readmission and chronic illness, severely impairs short- and long-term physical functioning and healthrelated quality of life, and diminishes overall and cancer-specific survival. Patients with a complicated postoperative course tend to be less physically fit preoperatively. Patients at risk for postoperative complications (i.e., high-risk patients) might benefit from prehabilitation. Therefore, further improvement of the perioperative process is warranted, in which the preoperative phase provides an opportunity. A patient's body composition and preoperative aerobic fitness might be used as preoperative risk assessment tools to diagnose and thereupon identify high-risk patients. Preoperative risk assessment remains of high importance to further improve outcome and adapt patient-tailored prophylactic treatment strategies (e.g., by selecting high-risk patients who may benefit from preoperative preventive (prehabilitation) interventions). Chapter $\mathbf{1}$ introduces the objective of this thesis forthcoming out of the abovementioned rationale, that is to find tools to screen, assess, and diagnose high-risk patients with colorectal cancer throughout the preoperative period (with the focus on physical fitness in the preoperative phase) and to investigate whether postoperative outcomes in these patients can be improved with a community-based exercise prehabilitation program.

The known 'traditional' risk factors that could assist in identifying patients with a potential risk for an impaired postoperative course, which are already regularly used as case-mix correction factors in the Dutch ColoRectal Audit (DCRA), are sex, body mass index (BMI), age, Charlson comorbidity score, American Society of Anesthesiologists (ASA) classification, preoperative tumor complications (e.g., fistula, obstruction, perforation, anaemia), urgency of the resection, tumor location, extensive resection due to locally advanced tumors or metastases, pathologic tumor ( $\mathrm{pT}$ ) stage, and pathologic metastasis (pM) stage. To gain more insight in the 'traditional' factors that are associated with postoperative complications and pathologic complete response ( $p C R$ : the absence of viable adenocarcinoma cells in the surgical specimen, including primary tumor and lymph nodes) in patients with locally advanced rectal cancer treated with neoadjuvant 
chemoradiation followed by surgery, we retrospectively aimed to identify and describe these prognostic factors in chapter 2 . Outcomes of this study can be used to help patients, their relatives, and the clinicians with determining prognosis and, thereupon, eventually select the proper treatment choices via shared decisions on interventions like prehabilitation. This study found that the postoperative complication rate was significant $(69 \%, n=68)$. Complications as an ileus and (perineal or abdominal) wound complications were most manifest. Age, intra- or postoperative blood transfusions, and PT stage predicted postoperative complications and/or postoperative grade 3-5 (severe) complications. The $\mathrm{pCR}$ rate was $22 \%(n=22)$. No independent factors predicting $\mathrm{pCR}$ were found on the base of this approach.

Besides the abovementioned 'traditional' risk factors, a patient's body composition and preoperative aerobic fitness might be additional or even more powerful preoperative risk assessment tools to diagnose and thereupon identify high-risk patients. Therefore, in chapter 3, the associations between body composition (i.e., muscle mass, radiation attenuation, and sarcopenic obesity) as assessed by using the computed tomography (CT) scan and postoperative outcome after treatment of locally advanced rectal cancer were evaluated. We found that radiation attenuation, also known as muscle density or myosteatosis, was associated with overall and grade 3-5 postoperative morbidity. Moreover, sarcopenic obesity was associated with overall complications. This study underscored the importance of assessing body composition in the context of the diagnosis of patients with high risk of worse outcome after surgery. The herewith identified high-risk patients might benefit from preoperative optimization (prophylactic prehabilitation) to optimize perioperative health status and to consequently prevent a dismal course. Since imaging body composition via a CT scan is a usual ingredient of care in the work-up of all patients with rectal cancer, the advantage of using this parameter as a risk assessment tool is that no additional test is needed when considering body composition with regard to the preoperative screening, assessment, and interpretation of patient-related risk factors. As such, this test provides a relatively low-cost improvement of the perioperative trajectory.

Moreover, preoperative aerobic fitness, assessed by cardiopulmonary exercise testing (CPET), is a powerful preoperative risk assessment tool that has consistently been found to be able to independently predict postoperative outcome in patients undergoing intraabdominal surgery. Patients with a low preoperative aerobic fitness (oxygen uptake $\left(\mathrm{VO}_{2}\right)$ at the ventilatory anaerobic threshold (VAT) $<11 \mathrm{~mL} / \mathrm{kg} / \mathrm{min}$ ) are particularly at increased risk of postoperative complications. $\mathrm{VO}_{2}$ at peak exercise $\left(\mathrm{VO}_{2 \text { peak }}\right)$ and $\mathrm{VO}_{2}$ at the VAT are the most commonly used preoperative cardiopulmonary exercise test measures in studies recorded before major elective abdominal surgery. Since there are several clinical limitations when determining aerobic fitness using $\mathrm{VO}_{2 \text { peak }}$ and/or $\mathrm{VO}_{2}$ at the VAT, and since assessing only these two measures lead to substantial loss of physiological data, 
alternative objective (sub)maximal variables of aerobic fitness (e.g., the oxygen uptake efficiency slope [OUES]) might be useful for preoperative risk assessment. However, the validity of the OUES as a patient's effort-independent alternative measure of preoperative aerobic fitness, and its ability to predict postoperative outcomes of individual patients, were still unknown. Therefore, in chapter 4, we aimed to investigate the validity of the OUES as an effort-independent indicator of aerobic fitness of elderly patients scheduled for colorectal surgery, by correlating it with $\mathrm{VO}_{2 \text { eak }}$ and the $\mathrm{VO}_{2}$ at the VAT and by assessing its stability throughout the last part of the CPET. We showed that the OUES provides an objective and valid measure of aerobic fitness of these patients, as indicated by the high correlation coefficients with $\mathrm{VO}_{2 \text { peak }}$ and the $\mathrm{VO}_{2}$ at the VAT. Moreover, its ability to predict cut-off points for $\mathrm{VO}_{2 \text { peak }}$ and $\mathrm{VO}_{2}$ at the VAT might help to diagnose and discriminate (respectively in the context of the clinic and that of research) patients at higher and lower risk of postoperative morbidity.

Since the CPET is relatively expensive, time-consuming, and requires well-trained personnel for an adequate interpretation of its results, we were also interested in whether body composition parameters on CT scan could help to identify patients with a potentially low preoperative aerobic fitness. By doing so, we might be able to appropriately refer the right patients for performing a CPET. Therefore, in chapter $\mathbf{5}$, we evaluated the association between preoperative body composition and aerobic fitness of patients scheduled for colorectal surgery. We found that body composition variables of the included 78 patients were moderately associated with preoperative CPET-derived aerobic fitness. A higher ASA score and a higher visceral adipose tissue mass were associated with an increased risk of a $\mathrm{VO}_{2}$ at the VAT $\leq 11.1 \mathrm{~mL} / \mathrm{kg} / \mathrm{min}$ as a cut-off to classify patients as having an increased risk for postoperative morbidity. We concluded that the routinely performed abdominal CT scan cannot replace the CPET for preoperative diagnosis and/or risk assessment. However, it may contribute to the diagnosis, and gross preselection of the probably less physically fit fraction of patients which, after additional CPET or other valid fitness measurements, should be more exactly diagnosed for their perioperative health risks.

Next, we were interested whether the high-risk, less aerobically fit patients would benefit from taking part in preoperative exercise training as a part of prehabilitation in order to reduce the risks of developing postoperative complications. Previously was shown that a physical exercise training program prior to surgery (prehabilitation) can improve a patient's aerobic fitness. It remained to be seen whether prehabilitation also reduces postoperative complications, as most of the studies so far were rather underpowered, heterogeneous, and biased towards selection of patients with low risk of postoperative complications. However, prehabilitation might improve postoperative outcomes when the focus is more on the adequate selection of high-risk surgical patients and when the prehabilitation program is personalized, and probably multimodal, (partly) supervised 
at home or in a community-based setting, and when a patient's progress is objectively monitored. Therefore, we performed an adequately powered randomized controlled trial to evaluate the effects of a three-week community-based exercise prehabilitation program on 30-day postoperative complications with patients with a $\mathrm{VO}_{2}$ at the VAT $<11$ $\mathrm{mL} / \mathrm{kg} / \mathrm{min}$ planned for elective colorectal resection for colorectal cancer or dysplasia. In chapter $\mathbf{6}$ the study protocol was extensively described. In chapter $\mathbf{7}$, by executing this protocol, we showed that community-based exercise prehabilitation, as we hypothesized at the beforehand, reduced the risk of postoperative complications of high-risk patients scheduled for elective colon resection for (pre)malignancy. Probably, this is also true for high-risk patients undergoing rectal resection; however, the small number of patients with rectal cancer included in this study makes it impossible to draw conclusions about the effectiveness of prehabilitation for this specific patient group. Within a relatively short period of on average three weeks, prehabilitation improved aerobic fitness by approximately $10 \%$ and patients subsequently experienced a more than $40 \%$ decrease in the incidence of postoperative complications. We also found that patients with a lower preoperative hemoglobin level were at greater risk of postoperative complications. Consequently, we advised to have prehabilitation considered in each and every hospital and/or clinical guideline as usual care in high-risk patients scheduled for elective colon, and probably also rectal, surgery.

In chapter 8, the general discussion, the main results of this thesis are aggregated, interpreted, and discussed ('what's new') in the context of the existing clinical practice and literature ('what's known'). Furthermore, recommended steps to take in the (near) future ('what's next') are discussed. In what's known, we described the known risk factors that could assist in identifying patients with a potential risk for an impaired postoperative course after colorectal surgery, such as age, sex, and Charlson comorbidity score. Moreover, we described the outcomes of previous studies that aimed to investigate the role of body composition and aerobic fitness in predicting postoperative outcome. We know that reducing complications would considerably reduce the patient burden and costs. High-risk patients might benefit from preoperative physical exercise training. To date, exercise prehabilitation programs have been shown to improve physical fitness before hospitalization and surgery. However, evidence that this reduces postoperative complications still seems inconclusive. In what's new, we described the findings of our research projects. In what's next, we recommended to take the following steps in the near future: firstly, to implement risk assessment with aerobic fitness and body composition analyzed at the third lumbar vertebra (L3) level of the CT scan and use them as case-mix correction factors in the DCRA to make a more accurate comparison of outcome among various hospitals. Secondly, to further improve prehabilitation programs and implement prehabilitation as part of the new/innovative standard care. Thirdly, to focus on the effect of prehabilitation on the impact of a postoperative complication in individual patients and 
focus on the medium- and long-term effects of prehabilitation. Fourthly, to perform a costeffectiveness analysis. Fifthly, to investigate the prognostic value of the OUES for different postoperative outcomes in major abdominal surgery. And finally, to establish communitybased perioperative care networks and in-hospital prehabilitation departments. 


\section{Samenvatting}

De levensverwachting blijft stijgen en daarmee groeit het aantal ouderen in de bevolking snel. Met de vergrijzing van de bevolking zal ook de incidentie van kanker toenemen. Darmkanker staat op de derde plek van meest voorkomende kankers wereldwijd. Bij darmkanker is radicale resectie van de tumor, al dan niet met (neo)adjuvante behandeling, de hoeksteen van curatieve behandeling. Dit betreft over het algemeen een zware behandeling die een aanzienlijke impact op de patiënt heeft. De laatste jaren is niet alleen steeds meer aandacht voor het voorkómen en vroegtijdig opsporen van kanker (primaire en secundaire preventie), maar ook voor preventie in de perioperatieve zorg (tertiaire preventie), bijvoorbeeld aandacht voor het verminderen van (de impact van) postoperatieve complicaties en het voorkomen van een vertraagd of zelfs blijvend verlies van (fysiek) functioneren. Ondanks substantiële verbeteringen in de perioperatieve zorg in de afgelopen decennia, blijft het aantal complicaties in de gehele patiëntengroep na colorectale chirurgie aanzienlijk met percentages tot $30 \%$. Bij oudere, kwetsbare en niet fitte patiënten is dit percentage zelfs nog hoger, met percentages van ruim boven de $50 \%$. Een gecompliceerd postoperatief beloop kan het herstel vertragen of zelfs belemmeren, de ziekenhuisopnameduur verlengen, ongeplande heropname(s) en chronische ziekte(n) veroorzaken, het fysiek functioneren op korte en lange termijn en de gezondheid gerelateerde kwaliteit van leven ernstig schaden. Daarnaast kunnen postoperatieve complicaties de algehele en kanker-specifieke overleving verminderen. Patiënten met een gecompliceerd postoperatief beloop zijn preoperatief doorgaans fysiek minder fit. Juist deze patiënten hebben daardoor een verhoogd risico op postoperatieve complicaties (zogenaamde hoog-risicopatiënten) en kunnen baat hebben bij prehabilitatie. Daarmee biedt de preoperatieve fase kansen om een patiënt in een zo optimaal mogelijke conditie te brengen, om op die manier de kans op een gecompliceerd beloop te verkleinen. Momenteel is men met de bekende 'traditionele' risicofactoren onvoldoende in staat deze hoog-risicopatiënten adequaat te diagnosticeren en identificeren. Voor adequate risicoschatting zouden mogelijk de lichaamssamenstelling (zoals skeletspiermassa) en preoperatieve aerobe fitheid van een patiënt als instrumenten kunnen worden gebruikt. Preoperatieve risicoschatting is van cruciaal belang om de uitkomst van zorg verder te verbeteren, om modificeerbare risicofactoren te identificeren en optimaliseren en om preventieve interventies te initiëren, bijvoorbeeld door hoog-risicopatiënten te selecteren die baat kunnen hebben bij het preoperatief optimaliseren van de fysieke fitheid. Hoofdstuk 1 introduceert het doel van dit proefschrift dat voortkomt uit de bovengenoemde redenering, namelijk het vinden van instrumenten voor het screenen, beoordelen en diagnosticeren van hoog-risicopatiënten met een colorectale tumor gedurende de preoperatieve periode, met de nadruk op fysieke fitheid, en om te onderzoeken of de postoperatieve resultaten bij deze patiënten kunnen worden verbeterd met een prehabilitatieprogramma uitgevoerd in de eerstelijnszorg. 
De bekende 'traditionele' risicofactoren die kunnen helpen bij het identificeren van patiënten met een potentieel risico op een gestoord postoperatief beloop welke ook worden gebruikt als case-mix correctiefactoren in de Dutch ColoRectal Audit (DCRA), zijn geslacht, body mass index (BMI), leeftijd, Charlson comorbiditeitsscore, classificatie van de American Society of Anesthesiologists (ASA), preoperatieve tumorcomplicaties (bijvoorbeeld fistels, obstructie, perforatie, bloeding), urgentie van de resectie, tumorlocatie, uitgebreide resectie vanwege lokaal gevorderde tumoren of metastasen, pathologische tumor (pT) stadium en pathologische metastase (pM) stadium. Om meer inzicht te krijgen in deze 'traditionele' factoren die geassocieerd zijn met postoperatieve complicaties en pathologische complete respons ( $\mathrm{PCR}$ : de afwezigheid van levensvatbare adenocarcinoomcellen in het operatiepreparaat, inclusief primaire tumor en lymfeklieren) bij patiënten met lokaal gevorderd rectumcarcinoom behandeld met neoadjuvante chemoradiatie gevolgd door chirurgie, is retrospectief onderzocht of deze prognostische factoren geïdentificeerd konden worden in hoofdstuk 2. De resultaten van deze studie kunnen worden gebruikt om patiënten, hun naasten en clinici te helpen bij het bepalen van en informeren over de prognose na de operatie, alsook om vervolgens te komen tot een gezamenlijke besluitvorming over de behandeling ('shared decision-making') en over het initiëren van eventuele preventieve interventies zoals prehabilitatie. Uit deze studie bleek dat de postoperatieve complicaties significant waren $(69 \%, n=68)$. Complicaties zoals een ileus en (perineale of abdominale) wondcomplicaties waren het meest voorkomend. Leeftijd, intra- of postoperatieve bloedtransfusies en pT-stadium voorspelden postoperatieve complicaties en/of postoperatieve graad 3-5 (ernstige) complicaties. Het pCR-percentage was $22 \%(n=22)$. In deze studie werden geen onafhankelijke factoren gevonden die $\mathrm{PCR}$ voorspelden.

Naast de bovengenoemde 'traditionele' risicofactoren, kunnen de lichaamssamenstelling en aerobe fitheid van een patiënt aanvullende of zelfs krachtigere preoperatieve risicoidentificatie instrumenten zijn om hoog-risicopatiënten te diagnosticeren en identificeren. Daarom zijn in hoofdstuk $\mathbf{3}$ de associaties onderzocht tussen lichaamssamenstelling (skeletspiermassa, skeletspierdichtheid en sarcopene obesitas) bepaald met behulp van de computertomografie (CT) scan en postoperatieve uitkomst na de behandeling van een lokaal gevorderd rectumcarcinoom. De resultaten lieten zien dat skeletspierdichtheid, ook bekend als myosteatose, geassocieerd was met het optreden van complicaties (graad 1-5, alle complicaties) en graad 3-5 (ernstige) postoperatieve morbiditeit. Bovendien was sarcopene obesitas geassocieerd met het optreden van complicaties. De lichaamssamenstelling van een patiënt zou derhalve gebruikt kunnen worden om patiënten met een hoog risico op een slechtere uitkomst na een operatie te identificeren. Deze patiënten zouden vervolgens baat kunnen hebben bij preoperatieve interventies (prehabilitatie) om hun gezondheidstoestand te optimaliseren en een ongunstig beloop te voorkomen. Het voordeel van het gebruiken van lichaamssamenstelling als risico- 
identificatie instrument is dat er geen aanvullende tests of onderzoeken nodig zijn, aangezien een CT-scan behoort tot de standaard diagnostische work-up van patiënten met rectumkanker. Daarmee is het een relatief goedkope methode om mogelijk verbetering van het perioperatieve traject te bewerkstelligen.

Daarnaast is preoperatieve aerobe fitheid, beoordeeld met een cardiopulmonale inspanningstest (CPET), een krachtig preoperatief instrument voor risico-identificatie. Uit de literatuur blijkt aerobe fitheid een belangrijke voorspeller te zijn van de postoperatieve uitkomst van patiënten die een intra-abdominale operatie ondergaan. Vooral patiënten met een lage preoperatieve aerobe fitheid (zuurstofopname $\left(\mathrm{VO}_{2}\right)$ op de ventilatoire anaerobe drempel (VAT) $<11 \mathrm{ml} / \mathrm{kg} / \mathrm{min}$ ) lopen een verhoogd risico op postoperatieve complicaties. $\mathrm{VO}_{2}$ op de $\mathrm{VAT}$ en $\mathrm{VO}_{2}$ op maximale inspanning $\left(\mathrm{VO}_{2 \text { piek }}\right)$ zijn de meest gebruikte preoperatieve cardiopulmonale inspanningstestparameters in onderzoeken die zijn uitgevoerd voorafgaand aan grote electieve abdominale chirurgie. Aangezien er verschillende klinische beperkingen zijn bij het bepalen van aerobe fitheid met behulp van $\mathrm{VO}_{2}$ op de $\mathrm{VAT}$ en/of $\mathrm{VO}_{2 \text { piek }}$ (de patiënt is bijvoorbeeld door pijn of vermoeidheid niet in staat een maximale inspanning te leveren, waardoor een variabele niet bepaald kan worden) en omdat het beoordelen van alleen deze twee parameters leidt tot een aanzienlijk verlies van fysiologische gegevens, kunnen alternatieve objectieve (sub) maximale variabelen van aerobe fitheid (bijvoorbeeld de "oxygen uptake efficiency slope" [OUES]) nuttig zijn voor preoperatieve risico-identificatie. De validiteit van de OUES als een inspanningsonafhankelijke alternatieve parameter voor preoperatieve aerobe fitheid van een patiënt, en het vermogen om postoperatieve uitkomsten van individuele patiënten te voorspellen, waren echter nog onbekend. Daarom is in hoofdstuk $\mathbf{4}$ de validiteit van de OUES onderzocht als een inspanningsonafhankelijke parameter van de aerobe fitheid van oudere patiënten die gepland stonden voor colorectale chirurgie door naar de relatie te kijken met de bekende en veelgebruikte aerobe fitheidsparameters $\left(\mathrm{VO}_{2}\right.$ op de VAT en $\mathrm{VO}_{2 \text { piek }}$ ) en door de stabiliteit ervan te beoordelen gedurende het hele laatste deel van de CPET. Resultaten toonden aan dat de OUES een objectieve en valide parameter is voor de aerobe fitheid van deze patiënten, zoals blijkt uit de hoge correlatiecoëfficiënten met $\mathrm{VO}_{2}$ op de VAT en de $\mathrm{VO}_{2 \text { piek }}$. Daarnaast bleek de OUES in staat om afkappunten voor $\mathrm{VO}_{2} \mathrm{op}$ de VAT en $\mathrm{VO}_{2 \text { piek }}$ te voorspellen, en daarmee kan de OUES helpen bij het diagnosticeren en discrimineren (respectievelijk in de context van de kliniek en die van onderzoek) van patiënten met een hoger en lager risico op postoperatieve morbiditeit.

Aangezien de CPET relatief duur en tijdrovend is en goed opgeleid personeel vergt voor een adequate interpretatie van de resultaten, is in dit proefschrift ook onderzocht of lichaamssamenstelling gemeten op de CT-scan zou kunnen helpen bij het identificeren van patiënten met een mogelijk lage preoperatieve aerobe fitheid. Indien mogelijk kunnen zo de juiste patiënten doorverwezen worden voor het uitvoeren van een CPET. 
Daarom is in hoofdstuk $\mathbf{5}$ de associatie tussen preoperatieve lichaamssamenstelling en aerobe fitheid van patiënten die gepland stonden voor colorectale chirurgie geëvalueerd. De resultaten lieten zien dat de verschillende parameters van lichaamssamenstelling van de geïncludeerde 78 patiënten matig geassocieerd waren met preoperatieve aerobe fitheidsparameters afgeleid vanuit de CPET. Een hogere ASA-score en een hogere viscerale vetmassa waren geassocieerd met een verhoogd risico op een lage preoperatieve aerobe fitheid (dat wil zeggen een $\mathrm{VO}_{2}$ op de VAT $\leq 11,1 \mathrm{ml} / \mathrm{kg} / \mathrm{min}$, welke gebruikt wordt als afkapwaarde om patiënten te classificeren met een verhoogd risico op postoperatieve morbiditeit). Geconcludeerd kon worden dat de routinematig uitgevoerde abdominale CT-scan de CPET voor preoperatieve diagnostiek en/of risicoschatting niet kan vervangen. Het kan echter wel bijdragen aan de diagnose en grove voorselectie van de waarschijnlijk minder fysiek fitte patiënten die vervolgens nauwkeuriger moeten worden geanalyseerd om hun perioperatieve gezondheidsrisico's in kaart te brengen, bijvoorbeeld met behulp van een CPET.

Vervolgens is in een studie onderzocht of de hoog-risicopatiënten, degenen met een lage preoperatieve aerobe fitheid $\left(\mathrm{VO}_{2}\right.$ op de $\left.\mathrm{VAT}<11 \mathrm{ml} / \mathrm{kg} / \mathrm{min}\right)$, baat zouden hebben bij deelname aan een preoperatief trainingsprogramma (prehabilitatie) om het risico op het ontwikkelen van postoperatieve complicaties te verminderen. Eerder werd aangetoond dat prehabilitatie de aerobe fitheid van een patiënt kan verbeteren. Het bleef echter onduidelijk of prehabilitatie ook postoperatieve complicaties kon verminderen, aangezien de meeste onderzoeken tot nu toe een te lage statistische power hadden, heterogeen waren en vooral patiënten met een laag risico op postoperatieve complicaties includeerden. Uit een recente review bleek echter dat prehabilitatie de postoperatieve resultaten wel degelijk kan verbeteren wanneer de nadruk meer ligt op de adequate identificatie en vervolgens selectie van hoog-risicopatiënten in de studie, wanneer het prehabilitatieprogramma gepersonaliseerd is, en waarschijnlijk multimodaal, wanneer het programma (deels) onder toezicht thuis of in een eerstelijnspraktijk wordt uitgevoerd, en wanneer de vooruitgang van een patiënt objectief wordt gemonitord. Daarom is een gerandomiseerde gecontroleerde studie opgezet en uitgevoerd om de effecten van een drie weken durend prehabilitatieprogramma (uitgevoerd in de eerstelijn) te evalueren op de 30 dagen postoperatieve complicaties van hoog-risicopatiënten (dat wil zeggen patiënten met een $\mathrm{VO}_{2}$ op de VAT $<11 \mathrm{ml} / \mathrm{kg} / \mathrm{min}$ ) die gepland zijn voor een electieve colorectale resectie voor colorectale kanker of dysplasie. In hoofdstuk 6 is het onderzoeksprotocol uitgebreid beschreven. Hoofdstuk $\mathbf{7}$ laat zien, door dit protocol uit te voeren, dat prehabilitatie, zoals op voorhand verwacht, het risico op postoperatieve complicaties verminderde bij hoog-risicopatiënten die gepland waren voor een electieve colonresectie voor (pre)maligniteit. Waarschijnlijk geldt dit ook voor hoog-risicopatiënten die een rectumresectie ondergaan; het kleine aantal patiënten met rectumkanker dat in deze studie is opgenomen, maakt het echter onmogelijk om conclusies te trekken over de 
effectiviteit van prehabilitatie voor deze specifieke patiëntengroep. Binnen een relatief korte periode van gemiddeld drie weken verbeterde prehabilitatie de aerobe fitheid met ongeveer $10 \%$ en werd een reductie van bijna $50 \%$ gevonden in het optreden van complicaties bij de groep die fysiek getraind had. Daarnaast bleek dat patiënten met een lager preoperatief hemoglobinegehalte een groter risico hadden op postoperatieve complicaties. Het is daarom aan te bevelen om prehabilitatie in elk ziekenhuis en/ of klinische richtlijn als gebruikelijke zorg te overwegen bij hoog-risicopatiënten die ingepland zijn voor electieve colon- en waarschijnlijk ook rectumchirurgie.

In hoofdstuk 8, de algemene discussie, zijn in de context van de bestaande klinische praktijk en literatuur ('wat is bekend') de belangrijkste resultaten van dit proefschrift verzameld, geïnterpreteerd en besproken ('wat is nieuw'). Verder zijn aanbevolen stappen voor de (nabije) toekomst ('wat volgt') besproken. In 'wat is bekend', zijn de bekende 'traditionele' risicofactoren beschreven die kunnen helpen bij het identificeren van patiënten met een verhoogd risico op een gestoord postoperatief beloop na colorectale chirurgie, zoals leeftijd, geslacht en Charlson-comorbiditeitsscore. Bovendien zijn de resultaten van eerdere studies beschreven die de rol van lichaamssamenstelling en aerobe fitheid op het voorspellen van postoperatieve uitkomst hebben onderzocht. Het verminderen van complicaties en de impact daarvan bevordert niet alleen de kwaliteit van leven van de patiënt op korte en lange termijn, maar bespaart ook enorm veel kosten. Hoog-risicopatiënten kunnen baat hebben bij preoperatieve training. Momenteel is aangetoond dat prehabilitatieprogramma's de fysieke fitheid voorafgaand aan een ziekenhuisopname en operatie verbeteren. Het bewijs dat dit postoperatieve complicaties vermindert, lijkt echter nog steeds niet overtuigend. In 'wat is nieuw', zijn de bevindingen van de onderzoeksprojecten uit dit proefschrift beschreven. In 'wat volgt' zijn een aantal aanbevelingen gedaan voor de nabije toekomst. Ten eerste werd aanbevolen om risicoidentificatie met aerobe fitheid en lichaamssamenstelling (geanalyseerd op het niveau van de derde lumbale wervel (L3) op de CT-scan) te implementeren en deze te gebruiken als case-mix correctiefactoren in de DCRA om een nauwkeurigere vergelijking van de uitkomst tussen verschillende ziekenhuizen te maken. Ten tweede werd beschreven dat het verder verbeteren van prehabilitatieprogramma's en het implementeren van prehabilitatie als onderdeel van de nieuwe, innovatieve standaardzorg een vervolgstap kan zijn. Ten derde werd aanbevolen in toekomstig onderzoek meer te focussen op de middellange en langetermijneffecten van prehabilitatie en om niet alleen te focussen op het effect van prehabilitatie op het optreden van complicaties, maar ook op de impact van complicaties. Ten vierde is het uitvoeren van een kosteneffectiviteitsanalyse een aanbeveling. Ten vijfde werd aanbevolen de prognostische waarde van de OUES voor verschillende postoperatieve uitkomsten bij grote buikoperaties te onderzoeken. En tot slot is aanbevolen om perioperatieve zorgnetwerken en prehabilitatie-afdelingen in het ziekenhuis of in de eerstelijn op te zetten. 



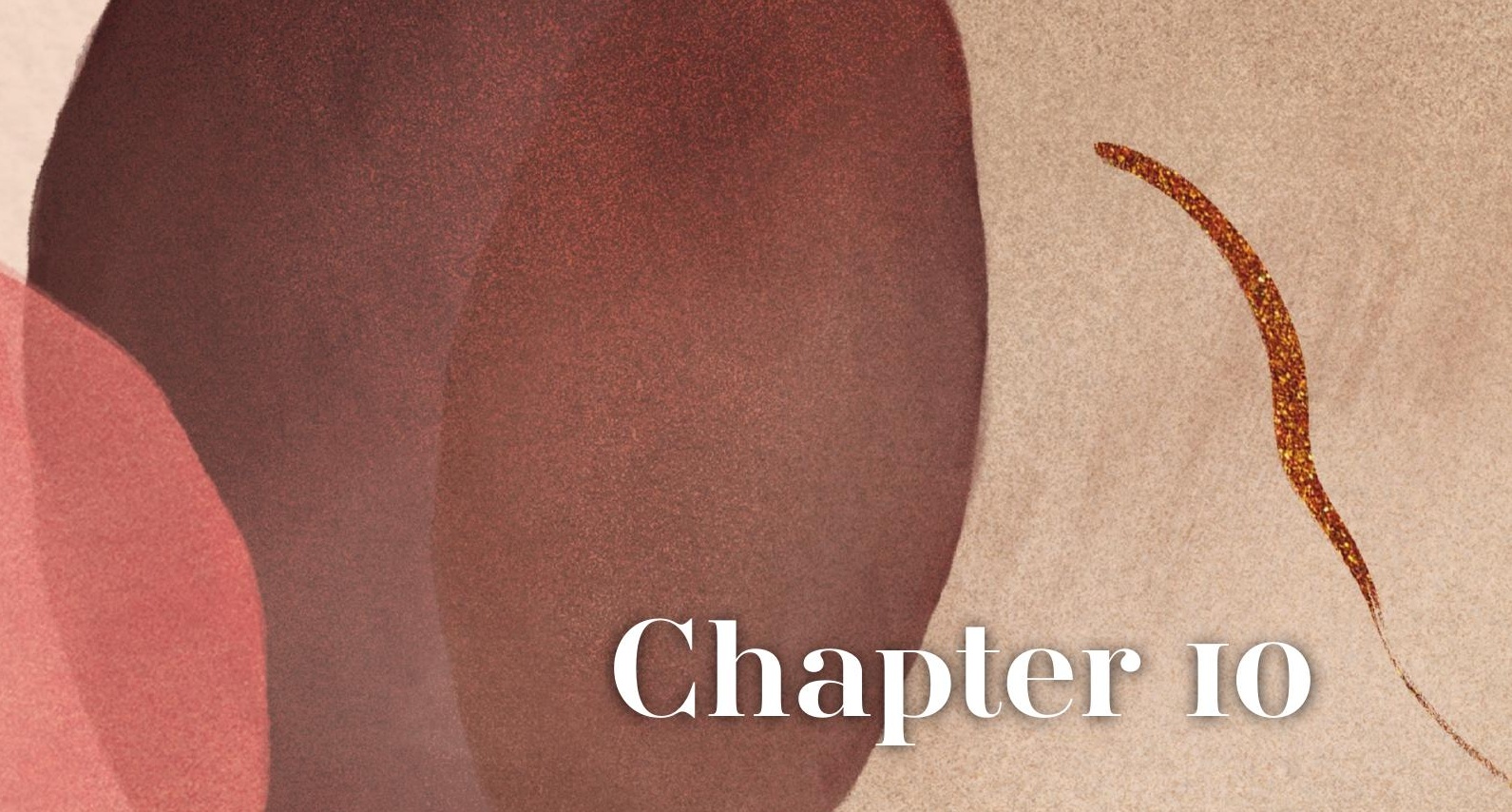

Impacl paragraph 


\section{Impact paragraph}

Colorectal cancer is the third most common cancer worldwide. With the further rise of a gradually aging population and without taken the further development and adoption of additional preventive precautions into account, the incidence of cancer is expected to increase. ${ }^{1}$ Such an increase may result in an expansion of health care demands, paralleled with increasing costs. Nowadays, the focus of public health interventions, more and more in coalition with interventions from the healthcare system, is not only on primary prevention of diseases like cancer (e.g., elimination of environmental pollution/ toxins, antismoking policies, healthy nutrition programs, educational activities that promote the healthy lifestyles), but also on secondary prevention (e.g., comprehensive screening strategies $)^{1}$ and tertiary prevention. As such, the focus in perioperative care is on the prevention of (the impact and consequences of) postoperative complications and prevention of a delayed recovery or even permanent loss of (physical) functioning known under the term "hospitalization-associated disability". Nowadays, the overall complication rate after colorectal surgery, which remains the cornerstone of curative treatment for colorectal cancer, ${ }^{3}$ is still significant with percentages up to $30 \% \cdot{ }^{3-5}$ Patients at risk for postoperative complications (i.e., high-risk patients) might benefit from prehabilitation. ${ }^{6,7}$ Prehabilitation aims to increase a patient's health and fitness between diagnosis and surgery in order to reduce postoperative complications and their impact, and enhance recovery of (physical) functioning postoperatively. ${ }^{8}$ Adequate screening, assessing, diagnosing, and prognosing of patients that are potentially at risk for an impaired postoperative outcome are essential for successful personalized healthcarerelated prevention. Therefore, the main objective of this thesis was to find tools that can be used in the preoperative period to screen, assess, and diagnose patients with colorectal cancer who are at risk for postoperative complications (with the focus on physical fitness in the preoperative phase) and to investigate whether postoperative outcomes of these patients can be improved with a community-based prehabilitation program, here focused on the effectiveness of physical exercise training.

The research described in this thesis highlights the role of body composition, as assessed by using the computed tomography (CT) scan, as a tool to preoperatively diagnose and/ or identify patients scheduled for colorectal surgery who are at risk for postoperative complications. Reduced muscle quality and the combination of obesity and low skeletal muscle mass were found to be associated with postoperative complications. Body composition was found to be moderately associated with objectively assessed aerobic fitness of these patients during a cardiopulmonary exercise test (CPET). Moreover, we found that a relatively new aerobic fitness variable (the oxygen uptake efficiency slope, OUES) might be a valid diagnostic measure of aerobic fitness of elderly patients scheduled for colorectal surgery that can be objectively determined in all patients without requiring 
a maximal effort during a CPET. This OUES variable might also help to more appropriately identify those patients at higher risk of postoperative morbidity. Finally, we showed that a three-week community-based exercise prehabilitation program ('prehabilitation study') substantially reduced the number of patients with one or more complications in a group of high-risk patients scheduled for colon resection for (pre)malignancy.

\section{Relevance}

\section{Scientific impact}

The insights gained during these thesis projects led to several adaptations in the perioperative work-up and care in patients with colorectal cancer in the Medisch Spectrum Twente. Previously, a patient's aerobic fitness was not routinely assessed in the perioperative work-up and still the outcomes of such an assessment are not registered in the nationwide quality registry for colorectal cancer patients. Since these thesis projects started, a patient's aerobic fitness became more and more a routinely assessed parameter by using a CPET in our hospital, and also in other hospitals. In this way high-risk patients can be identified, as a low aerobic fitness achieved on the CPET indicates an increased risk for postoperative complications. The results of the CPET are used to inform the patient and his/her relatives/informal caregivers and to consequently make a well-informed shared decision about whether or not to participate in specifically targeted preoperative (physical exercise) prehabilitation interventions. Given our positive results of prehabilitation in reducing postoperative complications in high-risk patients undergoing colorectal surgery, the next step should be to implement prehabilitation (at least consisting of validated physical exercise training presented in this thesis) in the hospital's standard perioperative trajectory by use of a perioperative trajectory continuum of care (CoC) protocol. The presented and/or validated physical factors (CPET values such as the oxygen uptake at the ventilatory anaerobic threshold and the OUES, and body composition variables such as radiation attenuation and sarcopenic obesity) described in this thesis, together with other recent insights and evidence in improving perioperative care, should also be incorporated in this $\mathrm{CoC}$ protocol.

Given our results of the prehabilitation study, the function of the so-called passive "waiting time" between diagnosis and initiation of treatment (treatment interval) in relation to a patient's preoperative aerobic fitness should be transformed into a proactive preparation period. At the first-hand, that might sound rather complicated as "waiting time" is currently used as one of the performance indicators for the quality of hospital care..$^{9,10}$ Recommendations regarding the treatment interval differ widely and can even differ within countries, because fundamental evidence-based guidance is absent. ${ }^{9}$ According to the Dutch so-called Treeknormen, ${ }^{11}$ it is recommended to treat $>80 \%$ of patients with colorectal cancer within 5 weeks from pathological confirmation, and $100 \%$ within 7 weeks. Another Dutch guideline describes that treatment will ideally start within six weeks 
after their first visit at the outpatient clinic. ${ }^{12}$ As a result of these guidelines, healthcare professionals are not always motivated to delay surgery in order to preoperatively optimize the health and fitness of their patients. In the literature, ${ }^{13}$ however, it was found that treatment delay (with treatment intervals of even 8-9 weeks) was not associated with impaired long-term outcomes in patients with colon cancer. When hospital logistics are optimized and rescheduled (e.g., a shorter period between time of diagnosis and first visit at the outpatient surgical clinic), and a more optimal collaboration between all disciplines can be realized (as we showed in our prehabilitation study), the currently recommended short "waiting time" can be sufficiently transformed and thereupon regularly used as a "preparation period" in most patients. However, this interval might not be sufficiently long for all patients. ${ }^{9}$ We suggest that if optimization of a high-risk patient's preoperative condition is preferred (by the healthcare professional and patient), it must be possible to extend the treatment interval. Frequent objective monitoring of training progress is important and sufficient progress in aerobic fitness should be achieved before proceeding with surgery in these patients. By doing so, that what sounded rather complicated, can or even should be tackled in the interest of each individual patient on a personal base, preferably with help of expert logistic- and data-intelligence.

In our prehabilitation study, we experienced that quite a few patients declined to participate in the study because of transportation difficulties. In another study it was found that patients prefer a home-based preoperative physical exercise training program. ${ }^{14}$ So, to maximize participation rate, adherence, and effectiveness of a prehabilitation program in high-risk patients, we suggest that the exercise component of the prehabilitation program best can be performed in the patient's pre-existent living context in which the patient's informal support system should be involved. An advantage of home-based physical exercise training is that the training program can be combined with functional exercise training of activities of daily living of relevance for the individual patient (e.g., climbing their own stairs, walking the dog in their own environment), which also have been shown to have a beneficial effect on daily function of older people. ${ }^{15,16}$ After gaining this insight, a collaboration with Lode B.V. (Groningen, the Netherlands) was established in which cycle ergometers now can be delivered at a patient's home and instantaneously customized to their physical requirements. With the help of an experienced and dedicated community physical therapist, the (supervision of) training can be tailored to each patient's talents to train, to monitor their training progress, and to involve their informal support. With the experiences gained during this collaboration, Lode B.V. was able to further develop and optimize their cycle ergometers. By doing so, we were able to develop quite a doable prehabilitation organization that delivers preventive means for high-risk patients at their home, an organization that is nowadays tested for its feasibility and pre- and postoperative success. ${ }^{17}$ 
The findings and experience gained in this thesis also led to the establishment of an experienced network of community physical therapists in the catchment area of the hospital, which, we think is crucial for an exercise prehabilitation program to be effective. Moreover, at the University Medical Center Groningen (UMCG) a prehabilitation department is being set-up, in which trained and competent healthcare professionals, along with the patient and the patient's informal support system, aim to perform an interdisciplinary preoperative risk assessment and assist the patient to get fit for surgery (executed in primary care). If prehabilitation has become standard care and common sense, risk screening and assessment can also be performed in the primary care. Additionally, the Dutch Society for Surgery established the Workgroup Prehabilitation to give direction to the implementation of prehabilitation in the Netherlands. ${ }^{18} \mathrm{~A}$ nationwide position paper considering prehabilitation in perioperative care is on the way, in which our results (Chapter 7) have been used. At the initiative of the Dutch Healthcare Authority (NZa), an innovation arrangement is obtained, whereby financing agreements have been made for prehabilitation in patients undergoing surgery for colon and rectal cancer. Within this arrangement that started on October 1, 2020, a payment title is developed for prehabilitation (Diagnosis Treatment Combination [DBC's] On Their Way to Transparency, DOT) in which also costs for the implementation and organization are covered. ${ }^{18}$ These developments will hopefully eventually make the implementation of prehabilitation successful, realistic, and sustainable.

\section{Social impact}

Previous research showed that the occurrence of a complication within the first 30 days after a major surgery, independent of the patient's preoperative risk, reduced median patient survival by $69 \% .{ }^{19}$ Postoperative morbidity is also associated with functional decline after surgery and decreased health-related quality of life. ${ }^{20}$ Identifying patients at risk for a complicated postoperative course (e.g., by the use of their preoperative body composition and aerobic fitness) and performing targeted preventive interventions such as prehabilitation may positively influence patient outcomes, the need of health care resources, and, consequently, costs.

Costs associated with the prevention of (the impact of) complications would probably outweigh costs of the care for the postoperative complications, and preventing complications would - most importantly in the context of patient-centered care considerably reduce the patient's physical, mental, and social burden. ${ }^{4}$ In the Netherlands, it was shown that the average total hospital costs (primary admission and after discharge up to 90 days) for patients undergoing colorectal cancer surgery ranged from $€ 3,403$ (least expensive 2.5\%) to $€ 79,953$ (most expensive 2.5\%). ${ }^{4}$ The average costs for one patient without complications is $\sim € 9,000$ versus $\sim € 11,500$ and $\sim € 27,000$ for one patient 
with minor and severe complications, respectively. The estimated costs of a multimodal prehabilitation program are $€ 969$ per patient (containing twelve physical therapy sessions, protein supplements, project management, and data collection). ${ }^{21}$ We therefore expect prehabilitation to be cost-effective. In future studies, these calculated figures should be investigated in a health technology assessment (HTA) approach in order to provide these assumptions with evidence and also with lessons to be learned from that.

\section{Target groups and activities}

First, the overall findings of this thesis are of value to patients and their informal caregivers. Adequate risk assessment prior to surgery can help them to gain insight in the patient's risks for an impaired postoperative course. This preoperative period can be used as a 'teachable moment', in which a patient might be more receptive towards lifestyle advices and more motivated to change their lifestyle than he or she would be in ordinary life. ${ }^{22}$ Moreover, these insights will help the patient, and also healthcare professionals, in shared decision-making for the choice and use of prophylactic (e.g., prehabilitation), surgical, and anesthetic interventions. Second, our findings can help healthcare professionals (e.g., physical therapists, surgeons, anesthesiologists, general practitioners) to diagnose patients at risk for an impaired postoperative course. They can offer these patients a targeted preventive intervention in order to optimize their health/fitness and the provided care. Third, scientists can use our findings in order to further improve prehabilitation programs for adequately selected low- and high-risk patients. Moreover, our findings will be of benefit for the development of a formal prehabilitation guideline.

The aforementioned target groups can be involved in and informed about the research results in a several ways. Firstly, patients can individually be informed about the research findings during their visit at the outpatient clinic. The results can be used to identify a patient's own (modifiable) risk factor(s) and, if necessary, a prehabilitation program can be offered to the patient. Secondly, gained knowledge during the research period of this thesis was, and will in the future be, shared with several hospitals and colleagues in (inter) national communities of practice in order to transfer/share knowledge and experiences. Thirdly, the research findings are and will be presented at (inter)national conferences. Fourthly, the results and experiences gained in this thesis project will be used to educate physical therapists in order to professionally guide and execute a prehabilitation program. Fifthly, scientific publications are posted and underway. Sixthly, the data gathered in this thesis project is available for (larger) studies, for example to perform a meta-analysis. Seventhly, the findings of this thesis project were, and will in the future be, used to contribute to an online course for multidisciplinary teams about frail high-risk patients and the role of prehabilitation. 


\section{Conclusion and ambitions}

In conclusion, the studies described in this thesis highlight the role of physical fitness (specifically aerobic fitness) as a means to preoperatively diagnose and/or select patients at high risk for postoperative complications after colorectal surgery and the role of exercise prehabilitation in reducing postoperative complications. We hope that our findings will be used to implement adequate risk assessment and prehabilitation in colorectal cancer care, to ultimately achieve the quadruple aim ${ }^{23}$ : improving patient experience, improving the health of a (population of) patient(s), reducing costs, and improving the well-being of health care professionals. 


\section{References}

1. World Health Organization. Men, Ageing and Health: achieving health across the life span. Available at: https://apps.who.int/iris/handle/10665/66941. Last accessed June 10, 2021.

2. Covinsky KE, Pierluissi E, Johnston CB. Hospitalization-associated disability: "She was probably able to ambulate, but I'm not sure". JAMA 2011; 306(16): 1782-93.

3. Dutch institute for clinical auditing (DICA) Leiden. DICA jaarrapportage 2018: Dutch ColoRectal Audit (DCRA). Available at: https://dica.nl/jaarrapportage-2018/dcra. Last accessed June 9, 2021.

4. Govaert JA, Fiocco M, van Dijk WA, et al. Costs of complications after colorectal cancer surgery in the Netherlands: Building the business case for hospitals. Eur J Surg Oncol 2015; 41(8): 1059-67.

5. Tevis SE, Kennedy GD. Postoperative Complications: Looking Forward to a Safer Future. Clin Colon Rectal Surg 2016; 29(3): 246-52.

6. Bongers BC, Punt IM, van Meeteren NL. On "Prehabilitation: The emperor's new clothes or a new arena for physical therapists?" Lundberg M, Archer KR, Larsson C, Rydwik E. Phys Ther. 2018;12:127-130. Phys Ther 2019; 99(7): 953-4.

7. Thomas G, Tahir MR, Bongers BC, Kallen VL, Slooter GD, van Meeteren NL. Prehabilitation before major intra-abdominal cancer surgery: A systematic review of randomised controlled trials. Eur J Anaesthesiol 2019; 36(12): 933-45.

8. Silver JK, Baima J. Cancer prehabilitation: an opportunity to decrease treatment-related morbidity, increase cancer treatment options, and improve physical and psychological health outcomes. Am J Phys Med Rehabil 2013; 92(8): 715-27.

9. Molenaar CJL, Winter DC, Slooter GD. Contradictory guidelines for colorectal cancer treatment intervals. Lancet Oncol 2021; 22(2): 167-8.

10. Dutch institute for clinical auditing (DICA) Leiden. Factsheet indicatoren Dutch ColoRectal Audit (DCRA) 2019. Available at: https://dica.nl/dcra/documenten. Last accessed May 26, 2021.

11. Overheid.nl. Parliamentary paper. 2003. Available at: https://zoek.officielebekendmakingen. nl/kst-25170-31.html. Last accessed May 6, 2021.

12. Stichting Oncologische Samenwerking (SONCOS). Multidisciplinaire normering oncologische zorg in Nederland, normeringsrapport 2018. Available at: https://www.soncos.org/wpcontent/uploads/2018/02/Soncos_norm-rapp2018.pdf. Last accessed May 6, 2021.

13. Hangaard Hansen C, Gögenur M, Tvilling Madsen M, Gögenur I. The effect of time from diagnosis to surgery on oncological outcomes in patients undergoing surgery for colon cancer: A systematic review. Eur J Surg Oncol 2018; 44(10): 1479-85.

14. Ferreira V, Agnihotram RV, Bergdahl A, van Rooijen SJ, Awasthi R, Carli F, Scheede-Bergdahl

C. Maximizing patient adherence to prehabilitation: what do the patients say? Support Care Cancer 2018; 26(8): 2717-23. 
15. Siemonsma PC, Blom JW, Hofstetter H, van Hespen ATH, Gussekloo J, Drewes YM, van Meeteren NLU. The effectiveness of functional task exercise and physical therapy as prevention of functional decline in community dwelling older people with complex health problems. $B M C$ Geriatr 2018; 18(1): 164,018-0859-3.

16. de Vreede PL, Samson MM, van Meeteren NL, Duursma SA, Verhaar HJ. Functional-task exercise versus resistance strength exercise to improve daily function in older women: a randomized, controlled trial. J Am Geriatr Soc 2005; 53(1): 2-10.

17. Berkel AE, Van Wijk L, Bongers BC, et al. Study protocol of a single-arm pre-post study to assess the preliminary effectiveness and feasibility of a home-based bimodal prehabilitation program on preoperative aerobic fitness in high-risk patients scheduled for liver or pancreatic resection. Int J Clin Trials. 2020 May;7(2):103-111.

18. Nederlandse Vereniging voor Heelkunde (NVvH). Prehabilitatie. 2021. Available at: https:// heelkunde. $\mathrm{nl} /$ themas $/$ thema?dossierid $=56164352 \&$ title=Prehabilitatie. Last accessed May 12, 2021.

19. Khuri SF, Henderson WG, DePalma RG, Mosca C, Healey NA, Kumbhani DJ, Participants in the VA National Surgical Quality Improvement Program. Determinants of long-term survival after major surgery and the adverse effect of postoperative complications. Ann Surg 2005; 242(3): 326,41; discussion 341-3.

20. Moran J, Guinan E, McCormick P, et al. The ability of prehabilitation to influence postoperative outcome after intra-abdominal operation: A systematic review and meta-analysis. Surgery 2016; 160(5): 1189-201.

21. Slooter GD, van den Heuvel B, Klaase JM, Dekker JWT. Innovatie Regeling Prehabilitatie. Werkgroep Prehabilitatie. NTvH 2020; 29(6): 24-5.

22. Brust M, Gebhardt WA, Numans ME, Kiefte-de Jong JC. Teachable moments: the right moment to make patients change their lifestyle. Ned Tijdschr Geneeskd 2020; 164: D4835.

23. Bodenheimer T, Sinsky C. From triple to quadruple aim: care of the patient requires care of the provider. Ann Fam Med 2014; 12(6): 573-6. 



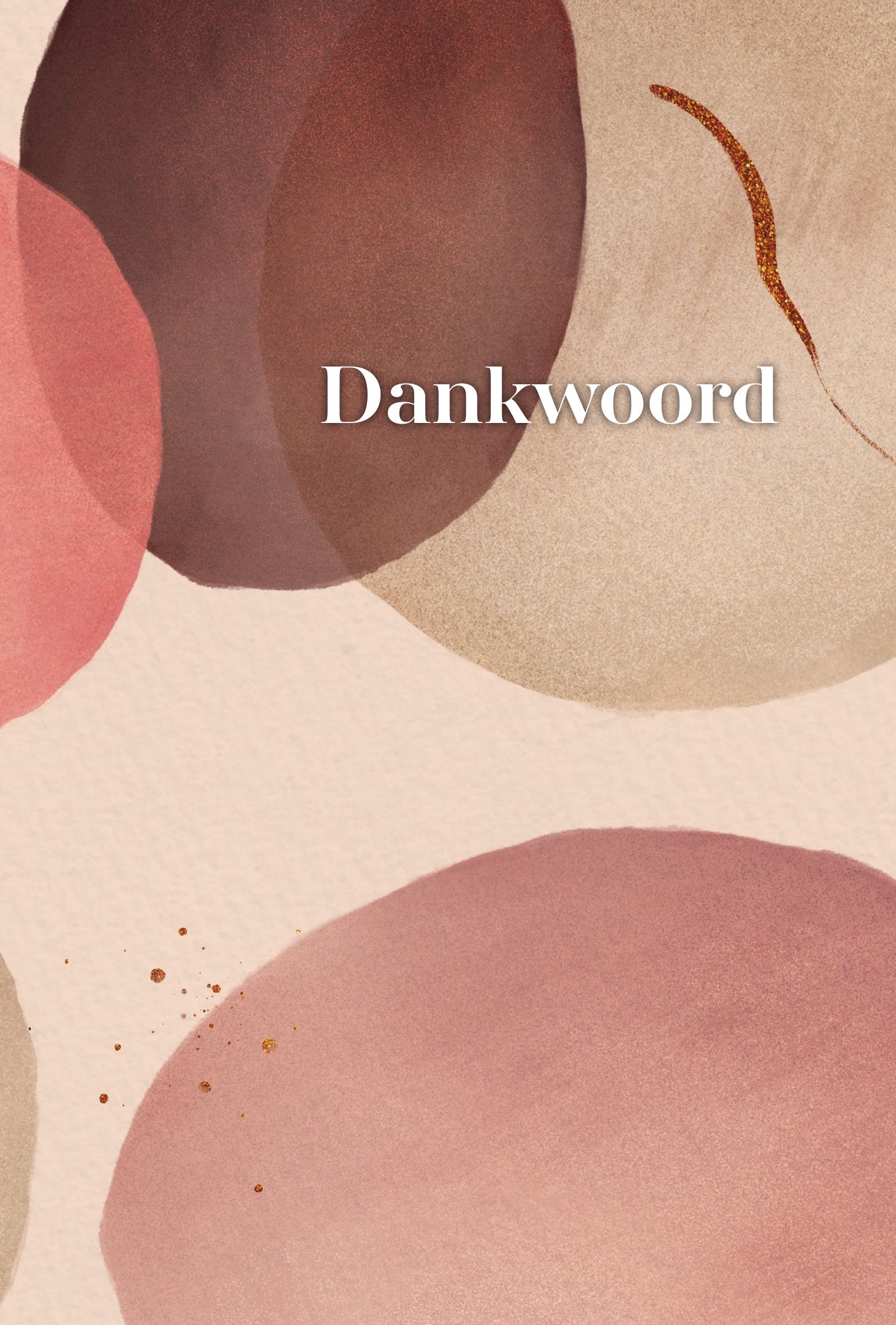




\section{Dankwoord}

Het is klaar! Ruim zes jaar geleden begon ik aan dit avontuur. Als arts-assistent op de chirurgie maakte ik destijds bijna dagelijks mee wat de impact van een gecompliceerd beloop na darmkankerchirurgie kon zijn. Vaak dacht ik: 'Wat zou het mooi zijn om patiënten zo'n gecompliceerd beloop te besparen'. Het onderwerp van mijn promotie was mij dan ook op het lijf geschreven. Zonder een heleboel inspirerende mensen om mij heen was dit avontuur niet geslaagd geweest. Daarom: hartelijk bedankt! Een aantal mensen wil ik graag in het bijzonder noemen.

In de eerste plaats wil ik graag alle patiënten die mee hebben gedaan aan het onderzoek bedanken. Eén ding is zeker, zonder jullie was er geen proefschrift geweest. In een kwetsbare periode waren jullie bereid deel te nemen aan het onderzoek. Daar heb ik veel bewondering en respect voor.

Hooggeleerde heer Van Meeteren, beste Nico, aanvoerder van ons prachtige team. In 2015 is onze samenwerking van start gegaan. Gelijk vanaf het eerste contact heb ik onze samenwerking als zeer prettig ervaren. Ik heb veel geleerd van jouw enthousiasme, kritische blik, ideeënstroom, enorme netwerk, kennis en kunde. Je was altijd zeer snel in jouw communicatie. Ik heb me menig maal verwonderd over jouw drukke agenda, maar er was altijd tijd en ruimte voor overleg. Bedankt voor alles!

Hooggeleerde heer Klaase, beste Joost, meesterbrein in prehabilitatie en de motor achter mijn promotie. Dankzij jou ben ik dit promotietraject aangegaan. Aanvankelijk was je mijn copromotor, maar gedurende het promotietraject werd je mijn, zeer gewaardeerde, tweede promotor. Al vanaf het moment dat ik coassistent was, doen we samen onderzoek. In al die jaren heb ik veel van jou geleerd. Jouw kennis, rust, enthousiasme en betrouwbaarheid waren goud waard. Dank voor deze mooie tijd!

Weledelzeergeleerde heer Bongers, beste Bart, wat een duizendpoot ben jij! Ik heb veel respect voor hoe je alles combineert: je bent niet alleen medisch fysioloog, maar ook docent, copromotor, jonge vader, sportman, et cetera. Het was een plezier om met jou te werken. Als mijn copromotor was je zeer betrokken en zorgvuldig. Jij stond altijd voor mij klaar. Dat heb ik enorm gewaardeerd. Jouw (inspannings)fysiologische kennis is indrukwekkend en een zeer waardevolle aanvulling in ons team. Bedankt voor alles!

Geachte leden van de leescommissie, beste professor Buhre, Dejong, Stassen, Veenhof en Verhoef, bedankt dat jullie als kenners in dit vakgebied wilden plaatsnemen in mijn 
beoordelingscommissie. Bedankt voor de tijd en moeite die jullie hebben genomen om mijn proefschrift kritisch te lezen.

Geachte coauteurs, beste Dankert Woutersen, Job van der Palen, Feike de Graaff, Marjolein Brusse-Keizer, Laura van Wijk, David van Dijk, Sanne Prins, Steven Olde Damink, Marie-Janne van Kamp, Hayke Kotte, Paul Weltevreden, Frans de Jongh, Michiel Eijsvogel, Machteld Wymenga, Marloes Bigirwamungu-Bargeman en Marc van Det, bedankt voor het delen van jullie kennis en jullie bijdrage aan de beschreven studies in mijn proefschrift. In het bijzonder wil ik professor Van der Palen en fysiotherapeuten Hayke Kotte en Paul Weltevreden noemen. Beste Job, jij bent mijn statistiek held! Ik kon mijn statistische analyses altijd met jou doornemen (en immer onder het genot van een heerlijk kopje thee). Ik heb veel van jou geleerd. Beste Hayke en Paul, zonder jullie was er geen patiënt getraind geweest. Jullie zijn vanaf het allereerste begin betrokken geweest bij de opzet en uitvoering van de prehabilitatiestudie. Dankzij jullie tomeloze inzet, kennis in de fysiotherapie, flexibiliteit, gedrevenheid en passie is de prehabilitatiestudie een succes geworden. Ik heb onze samenwerking als zeer fijn ervaren. Bedankt daarvoor!

Ook speciale dank aan de colorectaal chirurgen en arts-assistenten chirurgie uit het Medisch Spectrum Twente. Geachte chirurgen, beste Eino van Duyn, Pascal Steenvoorde, Walter Mastboom en Anneriet Dassen, bedankt voor de samenwerking rondom mijn colonspreekuur voor de prehabilitatiestudie. Ik heb veel van jullie geleerd. Beste artsassistenten Sander Paas (chirurg io), Sophie Gray (MDL-arts io), Suzanne Stokmans (chirurg io) en Marjolein Leemkuil (chirurg io), bedankt voor het overnemen van de prehabilitatiepoli op momenten dat ik dat zelf niet kon doen. Op die manier hebben jullie een zeer belangrijke rol gespeeld bij het includeren van patiënten.

Beste Anja Stam, als researchverpleegkundige bij de chirurgie in het Medisch Spectrum Twente had jij een spilfunctie bij de organisatie en uitvoering van de prehabilitatiestudie. Ik heb jouw bijdrage enorm gewaardeerd. Bedankt!

Ook wil ik de colorectaal chirurgen, arts-assistenten, oncologieverpleegkundigen, en researchverpleegkundige uit Ziekenhuisgroep Twente bedanken. Bedankt voor jullie bijdrage aan de inclusie van patiënten en bedankt voor de fijne samenwerking. I $k$ voelde me vanaf het eerste moment welkom in jullie team. In het bijzonder wil ik researchverpleegkundige Ilse Masselink noemen, bedankt voor jouw uitstekende coördinatie en dataverzameling rondom de prehabilitatiestudie in Ziekenhuisgroep Twente.

Beste HOED de Hagen huisartsen Eddy Rijk, Maarten van Bergeijk, Suzan van Vliet, Ellen Kerkhof en Nicole Nap, jullie zijn dan wel niet direct betrokken geweest bij mijn 
promotietraject, maar ik heb jullie interesse en bemoedigende woorden altijd erg gewaardeerd. Bedankt daarvoor! Jullie zijn hele fijne collega's om mee samen te werken.

Beste Better In, Better Out (BiBo) community of practice, bedankt voor alle leerzame avonden, presentaties en discussies.

Lieve familie en vrienden, ook aan jullie een speciaal woord van dank.

Lieve Bianca, Eva en Inge, vrienden voor het leven! We kennen elkaar inmiddels al meer dan de helft van ons leven. De afgelopen jaren hebben jullie voor de nodige afleiding en vooral voor een heleboel gezelligheid gezorgd. Geen moment met elkaar is saai. of we nou weekendjes weggaan, kerstmiddag vieren, samen eten, borrelen, twister spelen, samen sporten; het is altijd een feestje! Bedankt dat jullie er altijd voor mij zijn!

Lieve Ellen, Esther, Rianne, Tess en Sip, wat fijn om jullie als vrienden te hebben. We kennen elkaar van onze coschappen in Enschede en zijn inmiddels helemaal uitgewaaierd over Nederland. Desondanks zien we elkaar (gelukkig) regelmatig. Bedankt voor alle gezelligheid, leuke weekendjes weg, een top vakantie naar IJsland, etentjes, O\&N momenten samen, escape rooms, et cetera!

Lieve bootcamp-groep, Koos, Ilse, Kai, Shirley, Koen vd L, Lien, Eelke, Elianne, Koen D, Jeroen, Sintha en Bram, ooit begonnen we als bootcamp-groep, maar inmiddels geëindigd als vriendengroep. Bedankt voor de nodige afleiding tijdens al onze gezellige momenten samen, etentjes, weekendjes weg, bierfestivals, terrasjes, bruiloften, et cetera.

Mijn schoonouders, schoonzussen en zwagers, lieve Wilma, Leo, Erik \& Atsje, Marloes \& David, bedankt voor jullie interesse, liefde en betrokkenheid. Leo en Wilma, bedankt voor alle dagen oppassen zodat ik me helemaal kon focussen op mijn proefschrift. Dat heb ik enorm gewaardeerd! Ik kan me geen lievere schoonouders wensen.

Lieve papa, mama, zusjes en zwagers, lieve Ben \& Dorin, Denise \& Robbin, Carmen \& Jannik, Marlinde \& Michel, zonder jullie onvoorwaardelijke liefde en steun sta ik nergens. Papa en mama, mede dankzij jullie ben ik geworden wie ik ben. Jullie hebben mij respect, doorzettingsvermogen, discipline, loyaliteit, verantwoordelijkheid en betrokkenheid meegegeven. Jullie zijn mijn grote voorbeeld. Lieve zusjes en zwagers, wat een gezellige boel zijn we samen. Ik ben blij dat jullie mijn familie zijn!

En dan, mijn allergrootste steun en toeverlaat: Wouter. Lieve Wouter, jouw liefde, geduld, vertrouwen en betrokkenheid zijn ongeëvenaard. Jij hebt mij altijd enorm gesteund in mijn promotietraject. Ik prijs me erg gelukkig met jou. Naast het promoveren de afgelopen 
zes jaren, hebben wij ook op persoonlijk vlak de afgelopen jaren veel meegemaakt: we hebben samengewoond op $16 \mathrm{~m}^{2}$, we zijn (gelukkig al snel) verhuisd, we zijn getrouwd, we hebben een huis gekocht, jij kreeg een nieuwe baan bij Oost NL, ik werd huisarts, ik heb in de tussentijd ook zelf ervaren hoe het is om patiënt te zijn, en we kregen samen twee prachtige zoons. Bij al deze life-events had ik me geen betere man kunnen wensen. Bedankt!

Tot slot, Thom en Sven, jullie hebben de afgelopen jaren zéker als afleiding gezorgd. Wat houd ik van jullie! Jullie komst betekende ook dat ik niet elk vrij moment meer kon besteden aan mijn promotietraject. Ik dacht mijn proefschrift wel even te kunnen afronden tijdens mijn zwangerschapsverlof ('want baby's slapen toch vooral?'), maar dat bleek ijdele hoop. Zo klein als jullie nog zijn, hebben jullie ervoor gezorgd dat werk en privé meer in balans kwamen en dat organiseren en plannen inmiddels tot een van mijn grootste competenties behoren. 



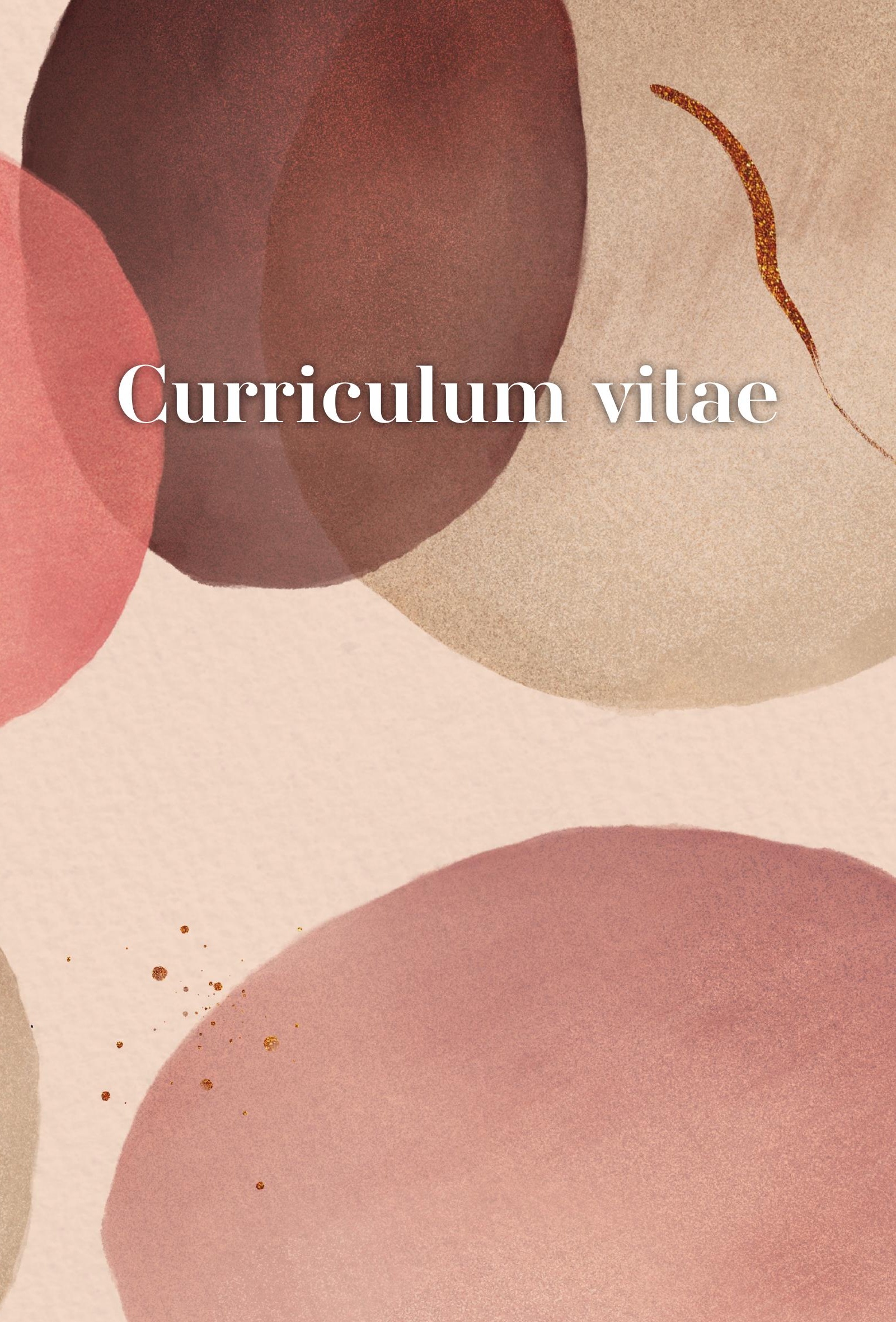




\section{Curriculum vitae}

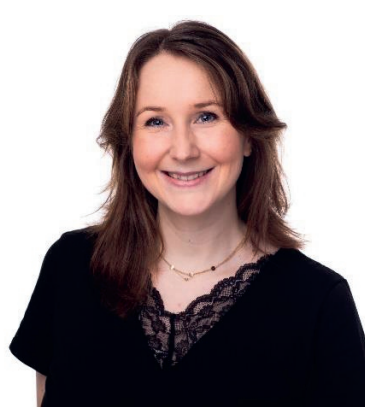

\section{Annefleur E.M. Berkel}

Annefleur Berkel was born on the $15^{\text {th }}$ of September 1988 in Enschede, the Netherlands. After completing secondary school (Gymnasium, the Grundel, Hengelo, 2000-2006) she studied Human Movement Sciences at the University of Groningen (2006-2009). With her bachelor's degree in Human Movement Sciences, she started her pre-master Medicine ('side entry') at the University of Groningen (2009-2010). In 2010, she started with the master Medicine at the University of Groningen, from which she graduated cum laude in 2013. In October 2013, she started working as a physician and researcher at the surgical department of the Medisch Spectrum Twente in Enschede, under supervision of Prof.dr. Joost Klaase.

In October 2015, she started her PhD project at the Department of Epidemiology within the Care and Public Health Research Institute (CAPHRI) at Maastricht University as an external $\mathrm{PhD}$ candidate. She carried out her research projects at the Medisch Spectrum Twente in Enschede. She combined her PhD project with working as a physician at the surgical department of the Medisch Spectrum Twente in Enschede, and later on as a physician in the elderly care at the nursing home the Cromhoff in Enschede.

In March 2016, Annefleur started her training to become a general practitioner, from which she graduated June 2019. Since then, she is working as a general practitioner in Twente, in which she integrated her research project and scientific knowledge.

In 2017, Annefleur married Wouter van der Kley, and together they have two sons (Thom 2019, Sven 2020). 
